\title{
Adopting Agile Software Development Practices: Success Factors, Changes Required, and Challenges
}

by

Subhas Chandra Misra

M.C.S. (Computer Science), University of New Brunswick, Fredericton, Canada M.Tech. (Computer Science), Indian Institute of Technology (IIT), Kharagpur

B.Eng. (Electronics \& Telecommunications Engineering), Andhra University, India

\author{
A thesis submitted to the \\ Faculty of Graduate Studies and Research \\ in partial fulfillment \\ of the requirements for the degree of \\ Doctor of Philosophy in Management \\ Carleton University \\ Ottawa, Ontario, Canada
}

(C) Subhas Chandra Misra

April 15, 2007 


$\begin{array}{ll}\begin{array}{l}\text { Library and } \\ \text { Archives Canada }\end{array} & \begin{array}{l}\text { Bibliothèque et } \\ \text { Archives Canada }\end{array} \\ \begin{array}{l}\text { Published Heritage } \\ \text { Branch }\end{array} & \begin{array}{l}\text { Direction du } \\ \text { Patrimoine de l'édition }\end{array} \\ \begin{array}{l}\text { 395 Wellington Street } \\ \text { Ottawa ON K1A ON4 }\end{array} & \begin{array}{l}\text { 395, rue Wellington } \\ \text { Ottawa ON K1A ON4 } \\ \text { Canada }\end{array}\end{array}$

Your file Votre référence ISBN: 978-0-494-27106-3 Our file Notre référence ISBN: 978-0-494-27106-3

NOTICE:

The author has granted a nonexclusive license allowing Library and Archives Canada to reproduce, publish, archive, preserve, conserve, communicate to the public by telecommunication or on the Internet, loan, distribute and sell theses worldwide, for commercial or noncommercial purposes, in microform, paper, electronic and/or any other formats.

The author retains copyright ownership and moral rights in this thesis. Neither the thesis nor substantial extracts from it may be printed or otherwise reproduced without the author's permission.
AVIS:

L'auteur a accordé une licence non exclusive permettant à la Bibliothèque et Archives Canada de reproduire, publier, archiver, sauvegarder, conserver, transmettre au public par télécommunication ou par l'Internet, prêter, distribuer et vendre des thèses partout dans le monde, à des fins commerciales ou autres, sur support microforme, papier, électronique et/ou autres formats.

L'auteur conserve la propriété du droit d'auteur et des droits moraux qui protège cette thèse. $\mathrm{Ni}$ la thèse ni des extraits substantiels de celle-ci ne doivent être imprimés ou autrement reproduits sans son autorisation.
In compliance with the Canadian

Privacy Act some supporting forms may have been removed from this thesis.

While these forms may be included in the document page count, their removal does not represent any loss of content from the thesis.
Conformément à la loi canadienne sur la protection de la vie privée, quelques formulaires secondaires ont été enlevés de cette thèse.

Bien que ces formulaires aient inclus dans la pagination, il n'y aura aucun contenu manquant.

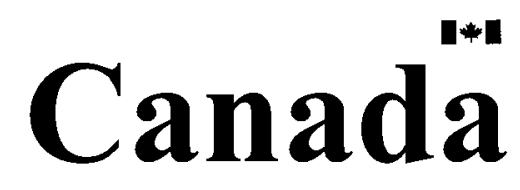




\section{Dedicated to my family}




\section{Acknowledgements}

I would like to acknowledge sincere gratitude to those people whose help, support, and encouragement enabled me to write this Doctoral Thesis. First and foremost, I am sincerely thankful to Professor Vinod Kumar, and Professor Uma Kumar for mentoring, encouraging, and supporting me at all stages of my Ph.D. program. Apart from providing extremely commendable advices for resolving the academic challenges involved with this Doctoral Thesis, they also provided me with extreme encouragement for encountering the other challenges that are typically faced during any doctoral program. They are always very eager to see me succeed and progress further. They have been parent-like figures for me, and will also remain as role models in my career.

I am grateful to Professor Roland Thomas for providing me valuable guidance with some of the Statistics related issues that I encountered while analyzing the survey data. The members of the Doctoral Thesis Committee, particularly Professor Aaron Nsakanda and Professor Shaobo Ji, deserve my special thanks for willing to painstakingly review this work and provide valuable comments. Sincere thanks are also due to Professor Lousie Heslop for all the support and encouragement she has been provided to me in different ways during the last few years. The administrative staff in the Sprott School, particularly Ms. Janice Walker and Ms. Anne Irvin, for the number of ways they helped me during the course of this degree program.

I am also thankful to the Ontario Government for providing me the Ontario Graduate Scholarship (OGS) during three consecutive years (2004-2005, 2005-2006, and 2006-2007), Carleton University for awarding me the Tuition Fee Waiver scholarship and the F.C. Mittal Award, and Eric Sprott School of Business for awarding me the Sprott Scholarship. I am also thankful to the organizers of the BIS 2003 (USA) for providing me a Best Student Paper Award for one of my published articles, which encouraged me to do research in this domain. I am also thankful to the Natural Sciences and Engineering Research Council of Canada (NSERC) for encouraging me to do further research in the area of Agile Software Development by offering me the prestigious NSERC Post-Doctoral Fellowship. 
I would like to thank all the survey participants who took their valuable time to respond to the survey questionnaire. During the course of the survey, several respondents even wrote words of encouragement regarding the work done in this Thesis. Since it is not possible to name each and all of them, I would take this opportunity to thank them all for their good words and support.

Finally, and most importantly, I would like to thank all my family members, for their continuous encouragement and support during the course of this Doctoral program. 


\begin{abstract}
Agile software development is an emerging approach in software engineering, initially proposed and promoted by a group of seventeen software professionals who practice a set of "lightweight" methods, and share a common set of values of software development. They consolidated their thoughts, and defined these methods as "agile". The approaches are based on experiences, and best practices from the past by the above-mentioned group of seventeen software professionals. As an emerging approach of this century, agile software development has undergone limited number of empirical studies.

In this Thesis, we advance the state-of-the-art of the research in this area by conducting survey-based ex-post-facto empirical (quantitative) studies by identifying the success factors from the perspective of software practitioners in agile software development projects, determining the key changes traditional projects have to undergo to adopt agile practices in their projects, and the challenges/risks they have to undergo for transition.
\end{abstract}

We describe theoretical frameworks we have developed to address our research questions, the hypotheses we have conjectured, the research methodology, the data analysis techniques we have used, and the results we have obtained from the data analysis.

The study was conducted using a survey-based methodology consisting of respondents who practice agile software development methodologies and who have experience practicing plan-driven software development in the past. The study indicates that nine of the fourteen hypothesized factors have statistically significant relationship with success. It also suggests a ranked list of changes required and challenges involved in adopting 
agile software development methodologies by projects practicing plan-driven software development. 


\section{Table of Contents}

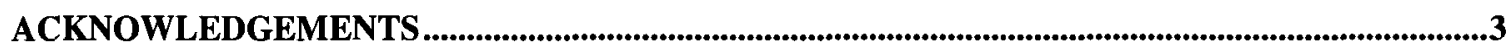

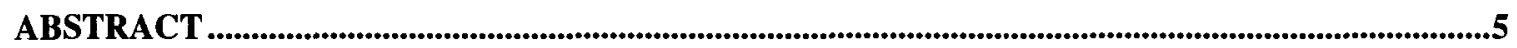

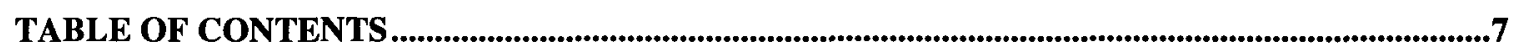

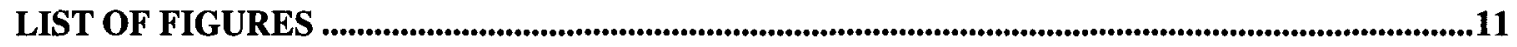

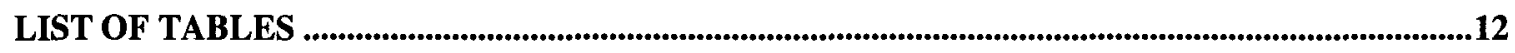

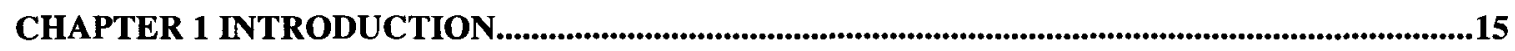

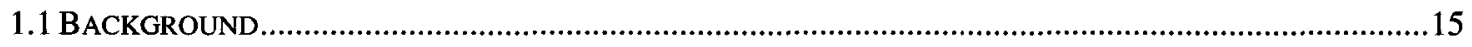

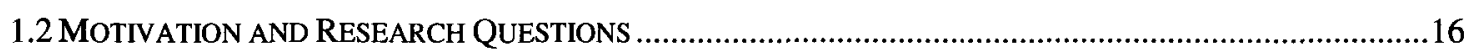

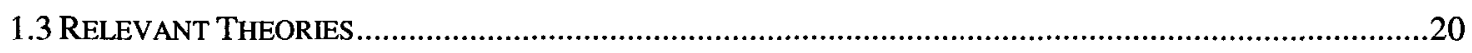

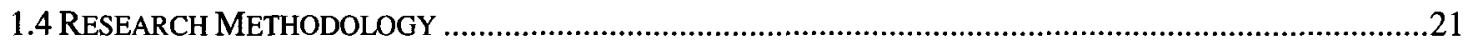

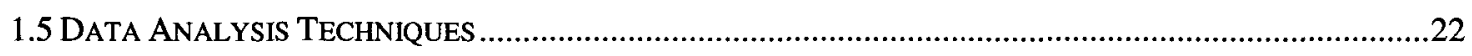

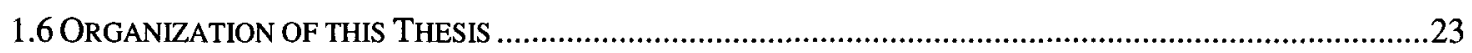

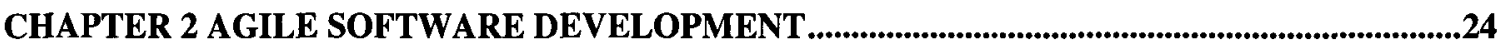

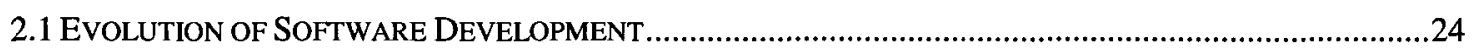

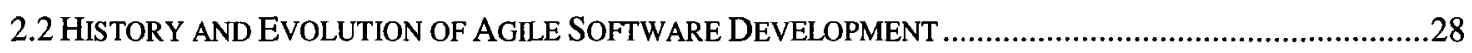

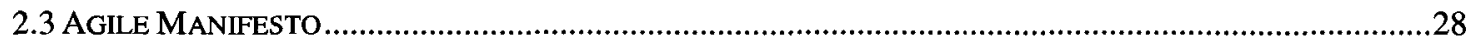

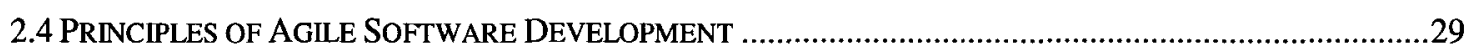

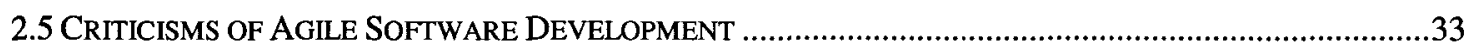

CHAPTER 3 SUCCESS FACTORS OF AGILE SOFTWARE DEVELOPMENT PROJECTS.........37

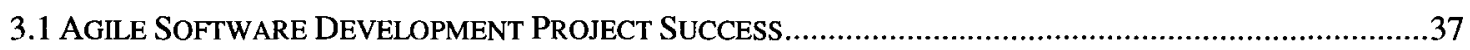

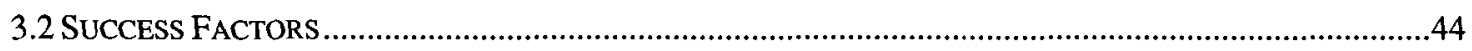

3.2.1 Organizational Factors .................................................................................................4

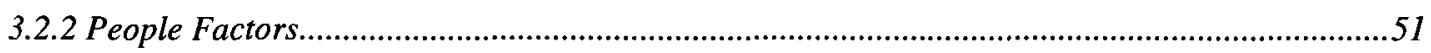

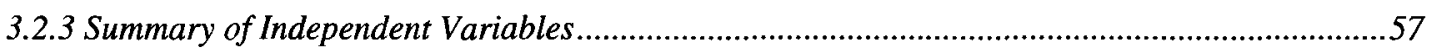

3.3 RESEARCH HYPOTHESES FOR IDENTIFYING THE SUCCESS FACTORS ...................................................60

CHAPTER 4 CHANGES AND CHALLENGES/RISKS IN ADOPTING AGILE PRACTICES IN TRADITIONAL SOFTWARE DEVELOPMENT PROJECTS..................................................................63

4.1 Home-Ground Characteristics: Rationale For CHANGE ..........................................................64 


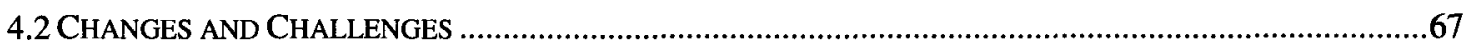

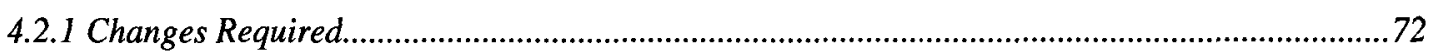

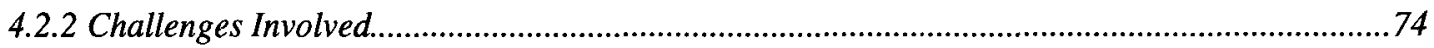

CHAPTER 5 RESEARCH METHODOLOGY ..............................................................................................81

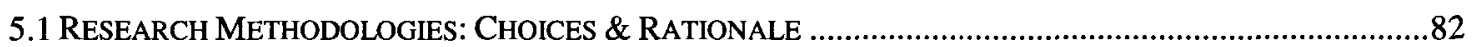

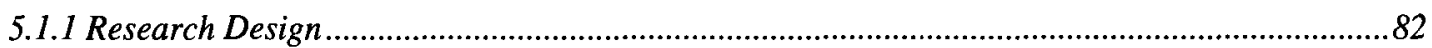

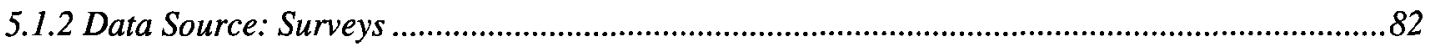

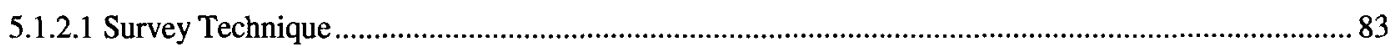

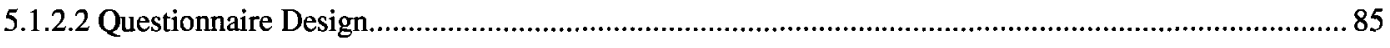

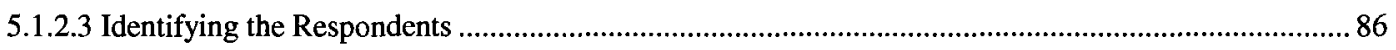

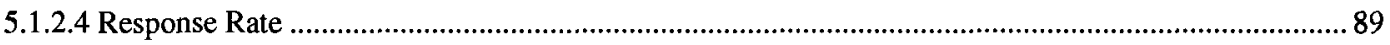

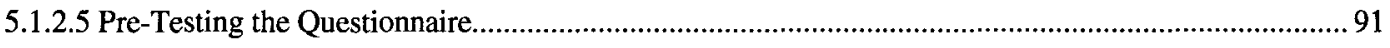

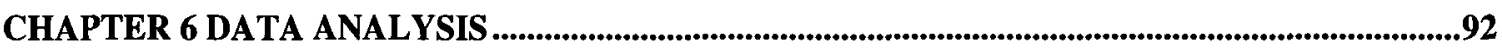

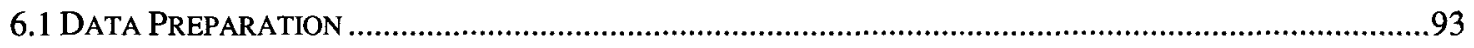

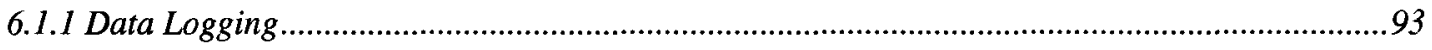

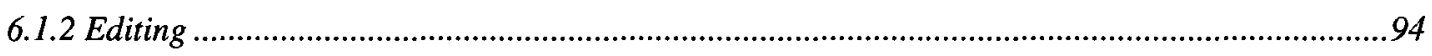

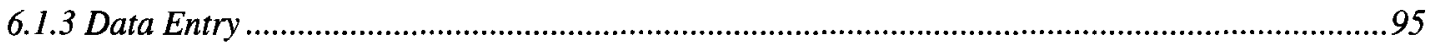

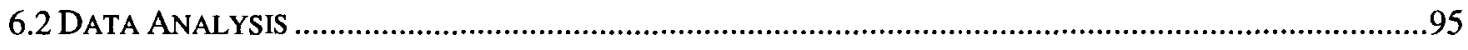

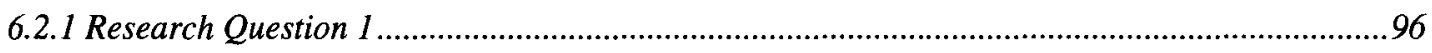

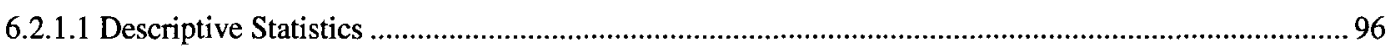

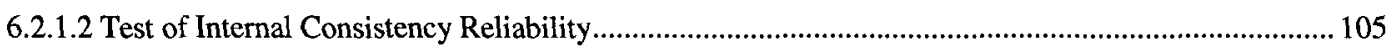

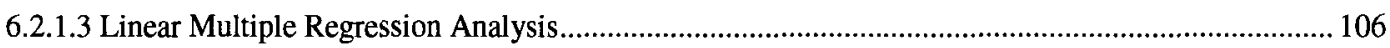

6.2.1.4 Success Factors Suggested by Respondents .......................................................................... 119

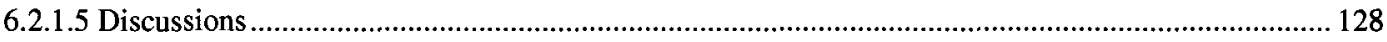

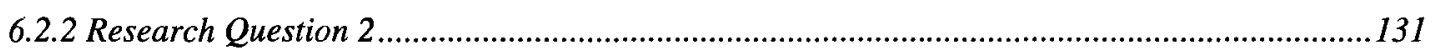

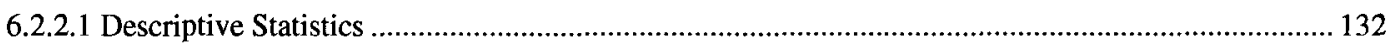

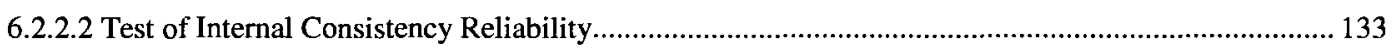

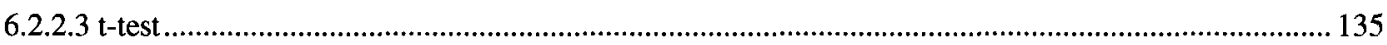

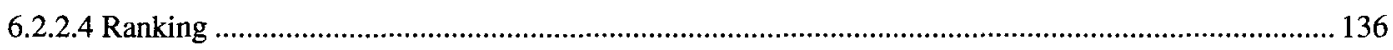

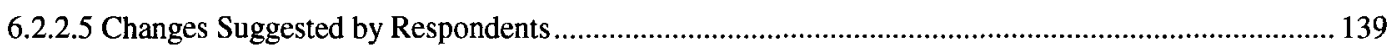

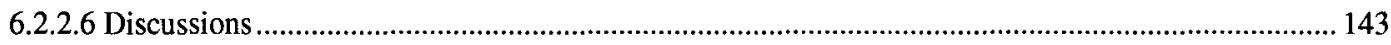

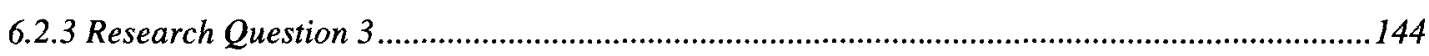

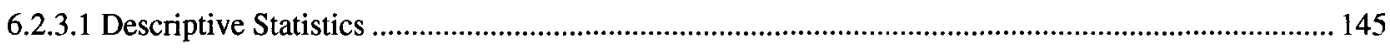

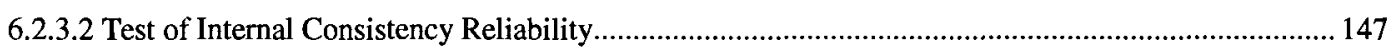

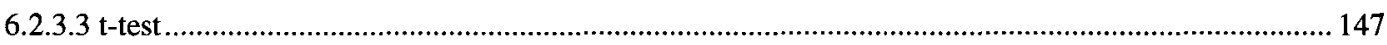

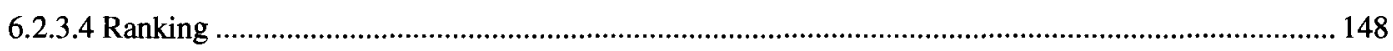

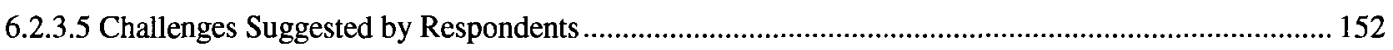




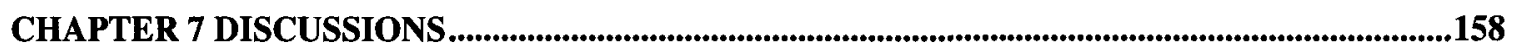

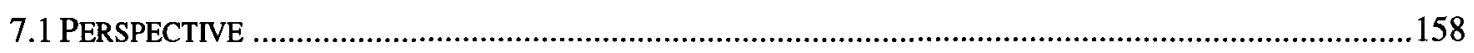

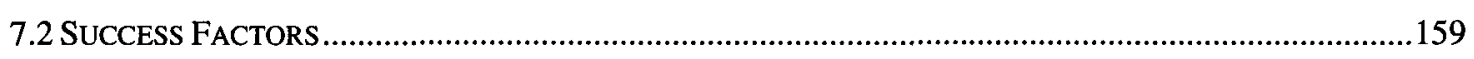

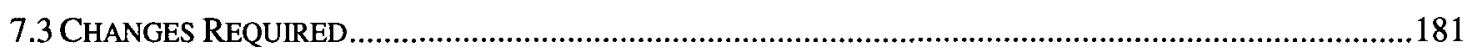

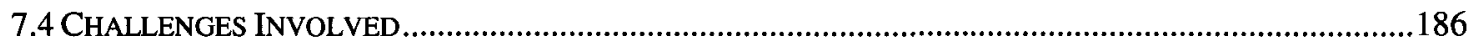

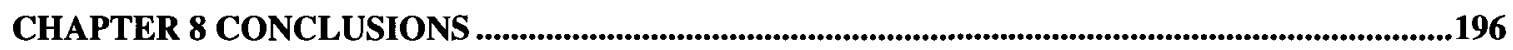

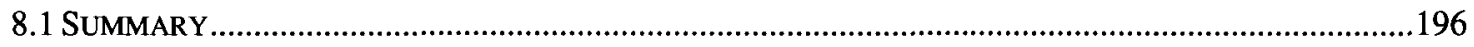

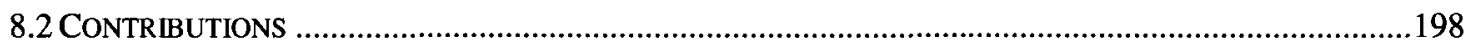

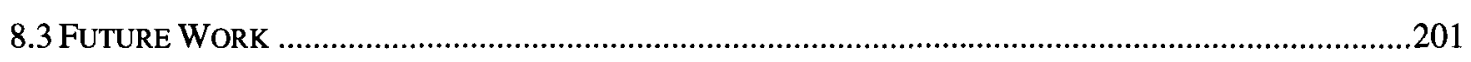

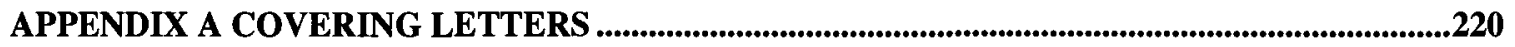

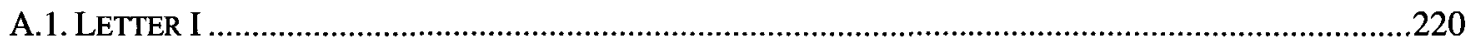

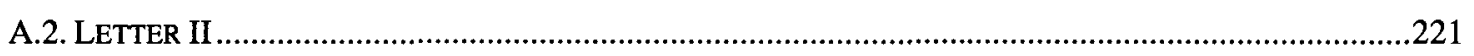

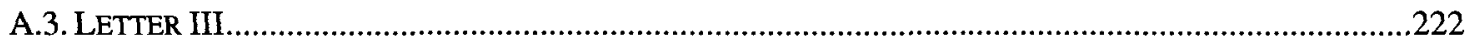

APPENDIX B SURVEY QUESTIONNAIRE .....................................................................................223

APPENDIX C LABELS OF VARIABLES USED IN THE STUDY ................................................230

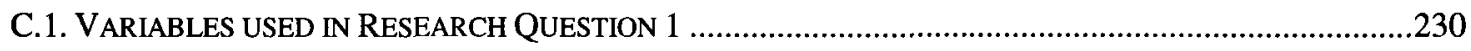

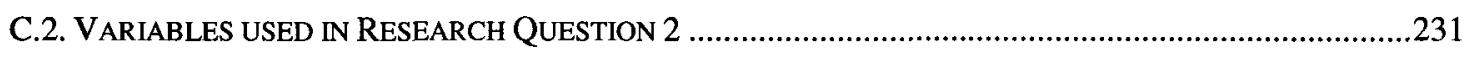

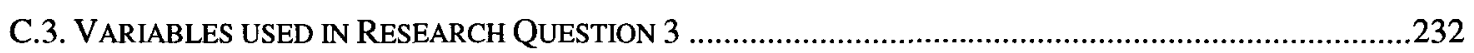

APPENDIX D FEEDBACK TO OPEN-ENDED QUESTIONS .............................................................234

D.1. LIST OF OTHER SUCCESS FACTORS OBTAINED FROM OPEN-ENDED QUESTIONS...............................234

D.2. LIST OF OTHER CHANGES OBTAINED FROM OPEN-ENDED QUESTIONS...........................................242

D.3. LIST OF OTHER CHALLENGES OBTAINED FROM OPEN-ENDED QUESTIONS ...................................245

APPENDIX E ADDITIONAL STATISTICAL RESULTS ..................................................................247

E.1. RESEARCH QUESTION 1: CONSOLIDATED DEPENDENT VARIABLE SUCCESS...................................247

E1.1. Detailed Descriptive Statistical Results ....................................................................................247

E1.2. Inter-Item Correlation Matrices for Variables Measured with Multiple Items.........................248

E. 1.3. Detailed Regression Analysis Tables ............................................................................249

E.2. RESEARCH QUESTION 1: INDIVIDUAL DEPENDENT VARIABLES MEASURING SUCCESS ....................253

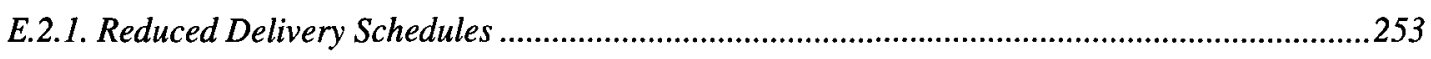

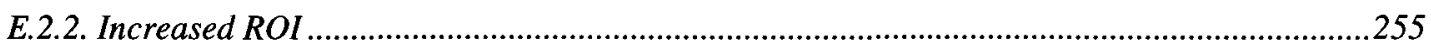

E.2.3. Increased Ability to Meet with Current Customer Requirements.............................................256 
E.2.4. Increased Flexibility to Meet with Changing Customer Requirements....................................258

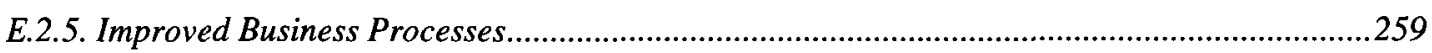

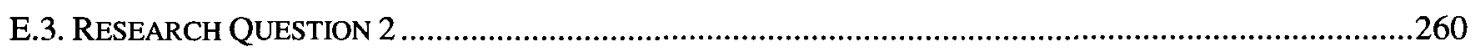

E.3.1. Detailed Results of Descriptive Statistics .............................................................................260

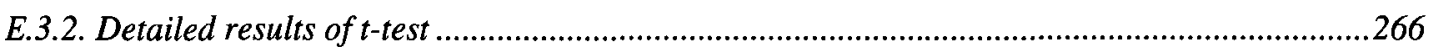

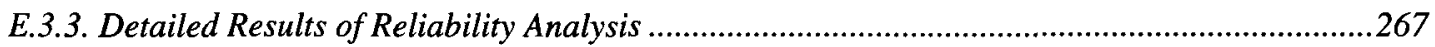

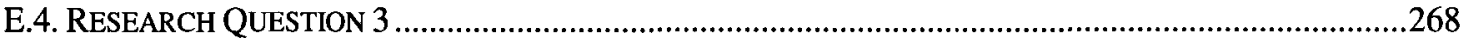

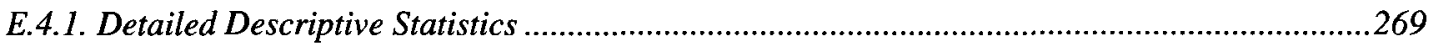

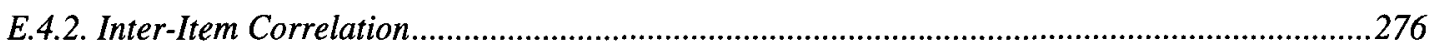

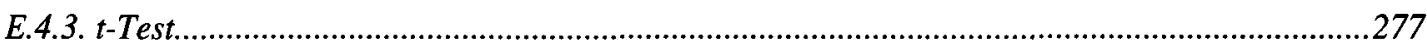

APPENDIX F SUMMARY OF SURVEY RESPONSES....................................................................................278

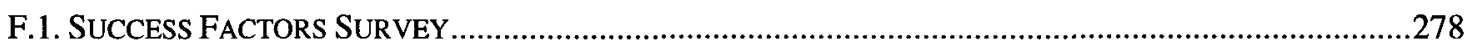

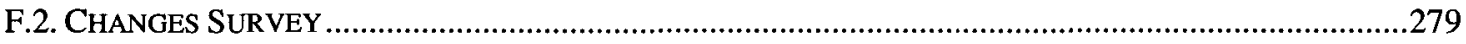

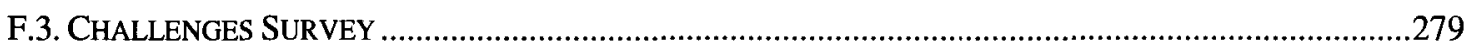




\section{List of Figures}

Figure 3.1: QUality/Time/Cost TRAdEOFF TRIANGLE IN SofTWARE DEVELOPMENT ..............................39

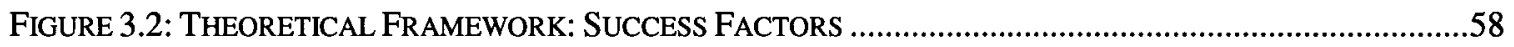

FigURE 4.1: TRANSITIONING TRAdITIONAL SOFTWARE DEVELOPMENT PROCESSES INTO AGILE.................69

FIGURE 4.2: THE CHANGES REQUIRED FOR ADOPTING AGILE PRACTICES IN TRADITIONAL PROJECTS, AND THE

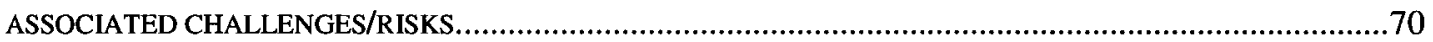

FIGURE 6.1: HistogRAm SHOWING FREQUENCY VERSUS REGRESSION STANDARdIZEd RESIDUAL ............116

FIGURE 6.2: NORMAL P-P PLOT OF REGRESSION STANDARDIZED RESIDUAL.................................................117

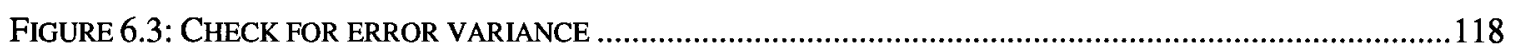

FIGURE 7.1: REVISED SUCCESS FACTORS FRAMEWORK ON THE BASIS OF SURVEY DATA ...........................160 


\section{List of Tables}

TABLE 3.1: A SUMMARY OF THE SUCCESS FACTORS AND THEIR RESPECTIVE ADVOCATES.

TABLE 4.1: HOME GROUNDS OF AGILE AND TRADITIONAL/PLAN-DRIVEN METHODS [TAKEN FROM BOEHM

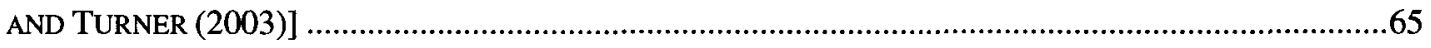

TABLE 6.1: RESEARCH QUESTION 1: SUMMARIZED DESCRIPTIVE STATISTICS ...........................................98

TABLE 6.2: PRIMARY IDENTITY OF THE RESPONDENTS' ORGANIZATIONS...................................................99

TABLE 6.3: THE NUMBER OF EMPLOYEES IN THE RESPONDENTS' ORGANIZATIONS....................................100

TABLE 6.4: THE NUMBER OF EMPLOYEES IN THE RESPONDENTS' TEAM ….............................................101

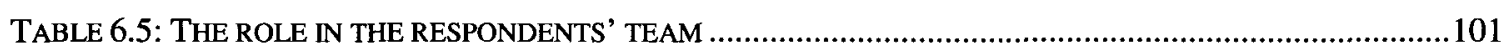

TABLE 6.6: DURATION FOR WHICH THE RESPONDENTS HAVE BEEN DEVELOPING SOFTWARE USING AGILE

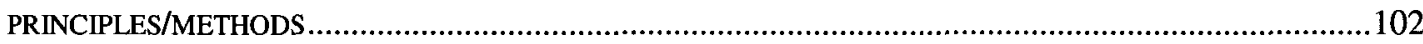

TABLE 6.7: DEGREE OF PRACTICE OF AGILE SOFTWARE DEVELOPMENT PRINCIPLES BY THE RESPONDENTS.

THE PERCENTAGE VALUES REPRESENT THE PERCENTAGE OF THE RESPONDENTS WHO RESPONDED WHO

SELECTED THAT OPTION IN THE 1 TO 5 LIKERT SCALE. ……..........................................................103

TABLE 6.8: RESEARCH QUESTION 1: ReLIABILITY STATISTICS SUMMARY ................................................106

TABLE 6.9: RESEARCH QUESTION 1: SUMMARIZED CORRELATIONS TABLE ............................................107

TABLE 6.10: RESEARCH QUESTION 1: REGRESSION COEFFICIENTS ....................................................112

TABLE 6.11: RESEARCH QUESTION 1: REGRESSION MODEL SUMMARY ....................................................112

TABLE 6.12: RESEARCH QUESTION 1: ANOVA TABLE FROM REGRESSION ANALYSIS................................115

TABLE 6.13: RESEARCH QUESTION 1: RESIDUALS STATISTICS FROM REGRESSION ANALYSIS .......................116

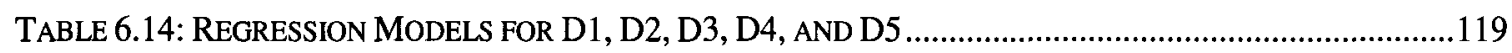

TABLE 6.15: RESEARCH QUESTION 2: SUMMARIZED DESCRIPTIVE STATISTICAL RESULTS ..........................133

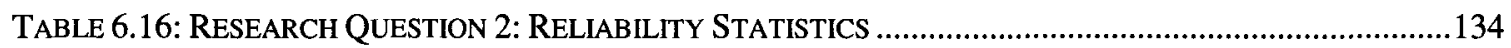

TABLE 6.17: RESEARCH QUESTION 2: INTER-ITEM CORRELATION MATRIX ...............................................134

TABLE 6.18: RESEARCH QUESTION 2: ONE-SAMPLE T-TEST .................................................................... 136

TABLE 6.19: RESEARCH QUESTION 2: RANKING OF THE CONSOLIDATED CHANGE ITEM CATEGORIES ........137

TABLE 6.20: RESEARCH QUeSTION 2: RANKING OF ALL THE INDIVIDUAL ChANGE ITEMS IN EACH CHANGE

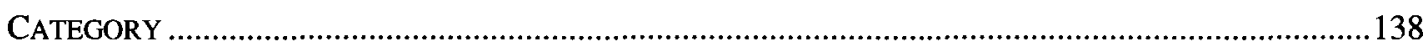

TABLE 6.21: RESEARCH QUESTION 3: SUMMARIZED DESCRIPTIVE STATISTICAL RESULTS .........................146

TABLE 6.22: RESEARCH QUESTION 3: RELIABILITY STATISTICS ...........................................................147

TABLE 6.23: RESEARCH QUESTION 3: SUMMARY ITEM STATISTICS.....................................................147

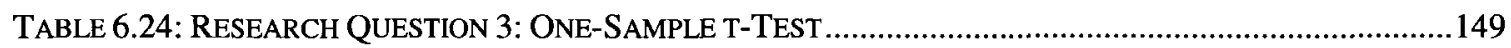

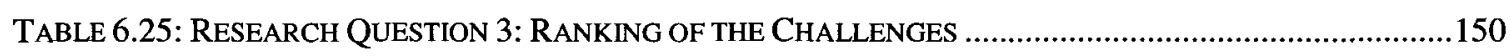

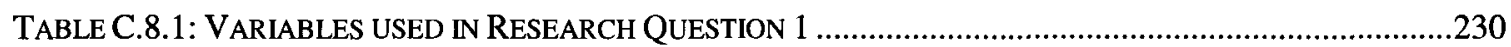

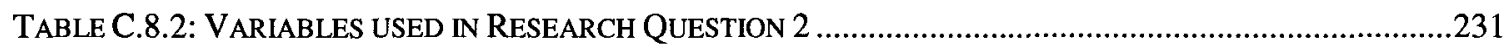




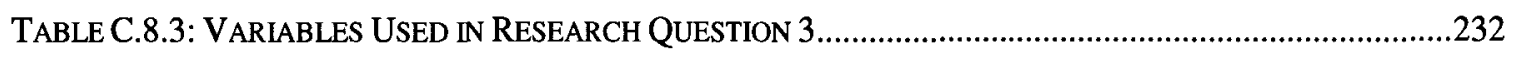

TABLE E.1: RESEARCH QueStion 1: DETAILED DESCRIPTIVE STATISTICAL RESULTS.................................247

TABLE E.2: RESEARCH QUESTION 1: INTER-ITEM CORRELATION MATRIX FOR ITEMS IN IND7 .....................248

TABLE E.0.3: RESEARCH QUESTION 1: INTER-ITEM CORRELATION MATRIX FOR ITEMS IN IND11 ...............248

TABLE E.4: RESEARCH QUESTION 1: INTER-ITEM CORRELATION MATRIX FOR ITEMS IN IND12 ...................248

TABLE E.5: RESEARCH QUESTION 1: INTER-ITEM CORRELATION MATRIX FOR ITEMS IN IND13 ...................248

TABLE E.6: RESEARCH QUESTION 1: INTER-ITEM CORRELATION MATRIX FOR ITEMS IN DEPENDENT .........249

TABLE E.7: RESEARCH QUESTION 1: DETAILED CORRELATIONS RESULTS ................................................250

TABLE E.8: RESEARCH QUESTION 1: VARIABLES ENTERED/REMOVED DURING STEPWISE REGRESSION ....251

TABLE E.9: RESEARCH QUESTION 1: EXCLUDED VARIABLES IN STEPWISE REGRESSION.............................252

TABLE E.10: RESEARCH QUESTION 1: REGRESSION MODEL SUMMARY FOR REDUCED DELIVERY SCHEDULES

TABLE E.11: RESEARCH QUESTION 1: ANOVA TABLE FROM REGRESSION ANALYSIS WITH REDUCED

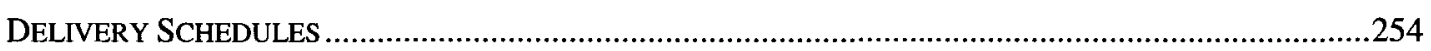

TABLE E.12: RESEARCH QUESTION 1: REGRESSION COEFFICIENTS FOR REDUCED DELIVERY SCHEDULES .254

TABLE E.13: RESEARCH QUESTION 1: REGRESSION MODEL SUMMARY FOR INCREASED ROI ......................255

TABLE E.14: RESEARCH QUESTION 1: ANOVA TABLE FROM REGRESSION ANALYSIS WITH INCREASED ROI

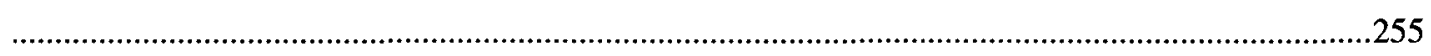

TABLE E.15: RESEARCH QUESTION 1: REGRESSION COEFFICIENTS FOR INCREASED ROI............................256

TABLE E.16: RESEARCH QUESTION 1: REGRESSION MODEL SUMMARY FOR INCREASED ABILITY TO MEET

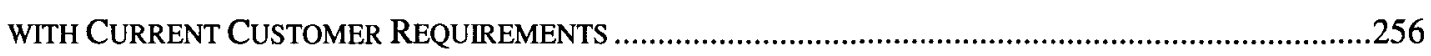

TABLE E.17: RESEARCH QUESTION 1: ANOVA TABLE FROM REGRESSION ANALYSIS WITH INCREASED

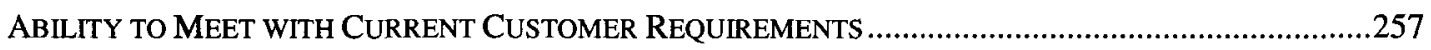

TABLE E.18: RESEARCH QUESTION 1: REGRESSION COEFFICIENTS TABLE FOR INCREASED ABILITY TO MEET WITH CURRENT CUSTOMER REQUIREMENTS

TABLE E.19: RESEARCH QUESTION 1: REGRESSION MODEL SUMMARY FOR INCREASED FLEXIBILITY TO

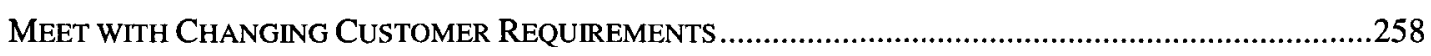

TABLE E.20: RESEARCH QUESTION : ANOVA TABLE FROM REGRESSION ANALYSIS WITH INCREASED FLEXIBILITY TO MEET WITH ChANGING CUSTOMER REQUIREMENTS ..................................................258

TABLE E.21: RESEARCH QUESTION 1: REGRESSION COEFHICIENTS FOR INCREASED FLEXIBILITY TO MEET

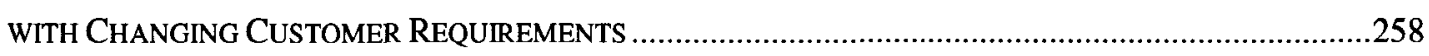

TABLE E.22: RESEARCH QUESTION 1: REGRESSION MODEL SUMMARY FOR IMPROVED BUSINESS PROCESSES

TABLE E.23: RESEARCH QUESTION 1: ANOVA TABLE FROM REGRESSION ANALYSIS WITH IMPROVED BUSINESS PROCESSES. .259 
TABLE E.24: RESEARCH QUESTION 1: REGRESSION COEFFICIENTS TABLE FOR IMPROVED BUSINESS

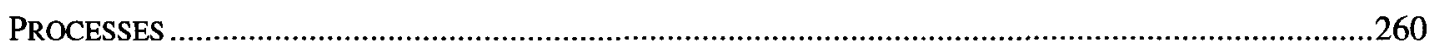

TABLE E.25: RESEARCH QUESTION 2: DETAILED DESCRIPTIVE STATISTICS (PART I) .................................260

TABLE E.26: RESEARCH QUESTION 2: DETAILED DESCRIPTIVE STATISTICS (PART II).................................262

TABLE E.27: RESEARCH QUESTION 2: ONE-SAMPLE TEST FOR ITEMS IN C1 ...........................................266

TABLE E.28: RESEARCH QUESTION 2: ONE-SAMPLE TEST FOR ITEMS IN C2 …......................................266

TABLE E.29: RESEARCH QUESTION 2: ONE-SAMPLE TEST FOR ITEMS IN C4 ............................................266

Table E.30: Research Question 2: Paired Samples Test with Consolidated Change ITEMS .........267

TABle E.31: RESEARCH QueSTION 2: PAIRED SAMPLES TEST WITH INDIVIDUAL CHANGE ITEMS ...............267

TABLE E.32: RESEARCH QUESTION 2: RELIABILITY STATISTICS FOR ITEMS IN C1 .....................................267

TABLE E.33: RESEARCH QUESTION 2: INTER-ITEM CORRELATION MATRIX FOR ITEMS IN C1 ....................267

TABLE E.34: RESEARCH QUESTION 2: RELIABILITY STATISTICS FOR ITEMS IN C2 ......................................268

TABLE E.35: RESEARCH QUESTION 2: INTER-ITEM CORRELATION MATRIX FOR ITEMS IN C2 .....................268

TABLE E.36: RESEARCH QUESTION 2: RELIABILITY STATISTICS FOR ITEMS IN C4 ….................................268

TABLE E.37: RESEARCH QUESTION 2: INTER-ITEM CORRELATION MATRIX FOR ITEMS IN C4 ......................268

TABLE E.38: RESEARCH QUESTION 3: DETAILED DESCRIPTIVE STATISTICS ..............................................269

TABLE E.39: RESEARCH QUESTION 3: INTER-ITEM CORRELATION MATRIX …………............................22

TABLE E.40: RESEARCH QUESTION 3: PAIRED SAMPLES T-TeST OF CHALLENGE VARIABLES ......................277 


\section{Chapter 1 \\ Introduction}

\subsection{Background}

Agile Software Development (ASD) is currently an emerging software engineering approach constituting of a set of principles initially advocated by a group of seventeen software practitioners, and now practiced by many software professionals. The principles they advocated leading to the emergence of the ASD philosophy are based on best practices and their previous success and failure experiences with many software development projects regarding what works and what does not. Each of these practitioners had their own different philosophies about how they approached software development. However, all of them advocated close collaboration between software development and business teams, as opposed to silo development by software teams; face-to-face communication, as opposed to over-emphasis on written documentation in projects; frequent delivery of portions of working software, as opposed to final delivery of the complete product at the end; accepting changing requirements by customers, as opposed to defining a fixed set of requirements "cast-in-stone"; and adaptive organizational capability of teams according to changing business requirements. These principles underlie the philosophy of $\operatorname{ASD}^{1}$ (Fowler and Highsmith, 2001; Fowler, 2002).

\footnotetext{
${ }^{1}$ Chapter 2 has been dedicated for further discussions about Agile Software Development.
} 
On the contrary, the traditional software development projects prior to agile were more focused on following well-defined plans and detailed documentations ${ }^{2}$. This will be further clear from the discussions in Chapter 2.

\subsection{Motivation and Research Questions}

In February 2001, these advocates of the agile philosophy met together, and prepare the Manifesto for Agile Software Development. Following $2001^{3}$, many other software practitioners were inspired by the philosophy behind ASD.

The overall goal of the Thesis is to improve the understanding of the emerging ASD approach using empirical studies involving projects that have adopted agile software development practices. In particular, we try to address the following research questions:

Research Question 1. What are the factors that will influence the success of projects that want to adopt agile software development practices from the perspective of agile software development practitioners?

Research Question 2. What are the important changes required for adopting agile software development practices in projects practicing traditional plan-driven software development? Can we rank them according to their level of importance?

\footnotetext{
${ }^{2}$ Throughout this Thesis, we use the terms "traditional development" and "plan-driven development" interchangeably. Since traditional development projects were plan-driven, they refer to the same thing. The use of these terminologies are consistent with the existing pieces of literature on agile software development.

${ }^{3}$ It should be clarified that the practices advocated by ASD are not newly identified in 2001 . Some of the practices were being followed by different practitioners for years in their projects. However, the development of the Agile Manifesto led to the emergence of a new approach advocated by practitioners
} 
Research Question 3. What are the most important challenges/risks that projects may encounter for adopting agile software development practices? Can we rank them according to their level of importance?

There is scarcity of literature reporting empirical studies concerning ASD projects. Although there are plenty of anecdotal experiences reported in the literature with agile practices, the number of empirical studies is limited to only a few. Some notable empirical studies can be found in Abrahamson and Koskela (2004), Baheti et al. (2002), Reiffer (2002), Rumpe and Schroder (2002), Kalermo and Rissanen (2002), and Shine Technologies (2003).

Reiffer (2002) is one of the first articles reporting survey results on agile methods. Although the nature of the survey is very simple, using only a small data space, it is important because it captures the experience of agile software engineers in various industry types. His studies report a productivity improvement, cost reduction, and timeto-market compression of agile practice adopters. He found that five firms reported that their defect rates from product were at par with their other projects.

Baheti et al. (2002) conducted empirical studies on distributed pair programming. They gathered empirical data in favor of pair programming in distributed teams, a very important issue in the current world of offshoring and outsourcing of IT business processes. Their study resulted in the creation of an special environment for distributed programming using dual screen projectors, and hypermedia-enhanced video streams. 
Rumpe and Schroder (2002) conducted a survey on Extreme Programming (XP) projects. They evaluated 45 questionnaires. They found out that the continuous presence of customers was important in XP projects. They also found out that although there are a plenty of problems in XP's success, it was found to be superior to some of traditional software development approaches. However, their survey had few limitations in terms of size, application domains, and seeded biases. They called for further surveys on XP.

Kalermo and Rissanen (2002) used an empirical case study in the context of agile inhouse software development in corporate venturing to find results supporting the principles and values of the Agile Manifesto (discussed in Chapter 2). Their study confirmed that tacit knowledge, motivation of employees, and their skills and knowledge levels are important in agile software development.

Shine Technologies (2003) conducted a simple survey of the global experiences with agile software development. They found that companies that follow agile practices have lower costs, better productivity, better quality, and better business satisfaction.

Abrahamson and Koskela (2004) performed a controlled case study on XP in practical settings. They did not set any hypothesis at the outset. In their study, they got four software engineers to implement a web-based system of 7698 lines of code over a period of 820 hours in eight weeks. They obtained favorable results in support of the usefulness of XP.

However, all of these studies are limited in their magnitude and exhaustiveness of investigation. 
Finally, let us summarize the motivations behind the work. Since its birth, ASD is increasingly becoming popular in the last few years. Because of the attractive claims of ASD, many organizations are switching to adopt agile practices in their software development projects. However, as this is an emerging software development philosophy, there are concrete empirical studies required to assess its different aspects. In this work, I am not assessing whether agile works or not. Rather what I am trying to investigate are the empirical evidences in support of the three research questions that I have documented earlier. Large scale empirical studies having the scope and magnitude of what has been performed as part of this Thesis work are scarce, if not unavailable in totality (availability of such work is not within my knowledge), and I am attempting to address this deficiency. What are more widely available are the experiential reports from different ASD practitioners while working in 1-2 projects.

The potential contributions are as follows. Let us assume that a software practitioner who used to practice plan-driven software development wants to adopt ASD practices in a project that is about to start. He/she would be interested to know:

(1) What are the factors on which his/her team should focus that would lead to the success of his/her project? There are potentially many factors to focus on. What is interesting to know are the critical success factors.

(2) What are the important changes to be focused on to adopt ASD practices?

(3) What are the important challenges he/she is likely to face while adopting ASD?

These are the issues that I have addressed in this work. My hope is that the work would aid the future software practitioners wanting to adopt ASD practices in their projects. This affirms the motivations behind the work. 


\subsection{Relevant Theories}

While there is no theory that can be firmly associated to our works (specifically, the exact research questions that I have considered), some of the "somewhat" relevant theories and their relevance to the works here are outlined below.

Perhaps the most relevant theory is the Theory of Constraints (ToC) (Goldratt, 1990; Goldratt, 1998; TOC 2). ToC aims to maximize the outcome of an activity or a set of activities having a common goal. It is often used in the context of manufacturing where the goal is to maximize the profitability from a manufacturing process by possibly decreasing the inventory and the operating expense, and increasing the throughput (Anderson 2003; TOC 1). As has been pointed out in the online article (TOC 1), the concept of ASD has parallelisms in ideas with that of the concept of ToC in that the agile methods (such as XP) aim at maximizing the outcome (more specifically, the number of features delivered in unit time), minimizing the inventory, and the number of steps required to achieve this (Anderson 2003, TOC 1). However, mapping software development processes with those of manufacturing has obvious limitations. We do not discuss them here as we will be digressing from our focal theme in this work.

Another theory, the Sociotechnical Systems Theory, suggests optimizing both the social and the technological systems for increasing the effectiveness of worksites (Cherns, 1976; Clegg, 2000, SST 1). It also emphasizes on self-directed work teams, in which teams are given autonomy to a considerable extent to control changes in work processes. It emphasizes percolating the communication and decision making from the higher levels in the organizational structure to the lower level. Thus these concepts have 
parallelism with some of the concepts behind ASD such as emphasis on customer collaboration, communication, and autonomy of teams over decision making.

Some of the concepts of ASD projects such as the dynamic and adaptive nature of such projects and their characteristic of being adaptive and constantly interacting with their environment have similarities in idea with the Open Systems Theory. The central idea of open systems theory when applied to management problems is that open system organizations are dynamic organizations that constantly interact with their environments. An open system organization has the ability to affect and be affected by the environment in which it operates. On the other hand, closed system organizations operate in fairly stable environments. Such organizations are self-contained to a great extent, and they do not interact constantly with their environment. The dynamic and constantly interacting nature of ASD projects is primarily the major resemblance one can draw with that of the open systems theory.

\subsection{Research Methodology}

We will design our research methodology based on the methodologies used in innovation diffusion and related streams of business and information systems (IS) research (e.g., Clover and Balsley 1979; Karahanna et al. 1999; Kumar et al. 2004; Maheshwari 2002; Maheshwari et al. 2005; Adrion 1993; Kontio 2001; Lazaro and Marcos 2005; Zelkowitz and Wallace 1998).

The nature of our research questions requires a post-facto design, wherein the parameters are not controlled. In order to address the first research question, we developed a theoretical (conceptual) framework based on previously published literature. 
This framework leads to the formulation of a set of hypotheses by keeping the research questions in mind. In order to address the second and the third research questions, we collected a set of items (changes/challenges) from the literature, and we wanted to rank the items based on survey responses. For addressing all the three research questions, a questionnaire is designed, and pre-tested before it is actually administered to the field for data collection. Then, data is collected and analyzed to assess the validity of the hypotheses. In our research, we survey multiple organizations that had adopted agile software development practices in the past.

The details of the research methodologies we use in this research are described in Chapter 5.

\subsection{Data Analysis Techniques}

The data that are collected from the surveys are statistically analyzed to validate the hypotheses formulated to investigate the research questions under consideration. We summarize below some of the statistical analysis methodologies we have used. Their details are provided in Chapter 6.

Descriptive statistical methods will be used for data exploration to gain an overall understanding of the nature of the data collected. The inter-question reliability will be tested using Cronbach's alpha test. This will help us to understand whether the responses to the different questionnaire items show high inter-question correlation. Correlation and regression analyses will be performed to understand the relationships between the dependent and independent variables. 


\subsection{Organization of this Thesis}

This Thesis is organized as follows. In addition to this introductory Chapter, there are six Chapters.

Chapter 2 is dedicated to introducing the fundamental concepts in Agile Software Development. It describes the evolution, manifesto and the principles behind agile software development. Chapters 3 and 4 are dedicated to describing the three research questions, relevant literature, the theoretical frameworks, and the formulated hypothesis that will be tested using survey data. Chapter 5 describes the research methodology. Chapter 6 describes the data analysis techniques that we used and the results that we obtained. Chapter 7 provides in-depth discussions of the results obtained. Finally, we conclude in Chapter 8 by summarizing the work, the main research contributions, and thoughts for future research. 


\section{Chapter 2 \\ Agile Software Development}

\subsection{Evolution of Software Development}

In this Section, we summarize some of important phases of the evolution of different software development methods. In the rest of the Chapter, we elaborate on how ASD came into being.

The very early software development methods include the code-and-fix method ${ }^{4}$, the stagewise method (Benington, 1956), the waterfall method (Royce, 1970), the evolutionary development method (McCracken and Jackson, 1982), the transform method (Balzer et al., 1983), and the spiral method (Boehm, 1988).

The code-and-fix method is one of the earliest available process models that were used in traditional software development. In this method, the software development activities are normally undertaken in two stages: writing the code, and then fixing the problems in the code. The major limitations of the code-and-fix method were the following. First, they made code fixes expensive after a few iterations of coding and fixing. Second, scalability using such a method became limited because there was no explicit recognition of planning, requirements, and design stages before actually undertaking the coding work for the incorporation of feedback from users.

The stagewise method was proposed by Benington in 1956. Unlike the code-and-fix method, software development in the stagewise method is conducted in different stages: 
operational plan, operational specifications, coding specifications, coding parameter testing, assembly testing, shakedown, system evaluation. The limitation of the stagewise method is that it does not have provisions for improving the latter stages of the software based on the experience gathered from the earlier stages. In 1970 , one of the most popularly used methods, called the waterfall method (Royce, 1970), was proposed with a two-fold improvement over the stagewise method (Royce, 1970; Boehm, 1988). The waterfall method uses feedback loops between successive stages of the software development process and it incorporates "prototyping" as an independent stage that would be conducted in concurrence with the early software development stages of requirements analysis and design, before undertaking the actual development of the software.

The waterfall method for software development remained a popular method for many years, as it was able to eliminate many of the limitations of the previous methods, and was used by different organizations for developing large-scale software. The waterfall method too was shown to have some limitations. While it worked well for the development of compilers, and operating systems, it had strong emphasis on document driven software development that was shown to be of limited help in the development of spreadsheets and small business applications. Additionally, excessive documentation of the specification of poorly understood applications, and their user interfaces was considered unnecessary.

${ }^{4}$ http://users.evtek.fi/ jaanah/DPAD/lectures/lec2/diff_m.html\#codeandfix 
McCracken and Jackson, in 1982, proposed the evolutionary software development method, in which the operational experience of users was incorporated in the software development lifecycle in its different stages. In this method, the users specify the functional characteristics of the initial operational software, and then feed their experience for determining subsequent product improvements. However, one of the limitations of this approach is that it is often difficult to change the users' existing software to incorporate the additional changes. In other words, using such a process, the maintenance and evolution of operational software can be a challenge.

The above methods still lacked the flexibility of easily modifying code through repeated re-optimizations as such code would often become poorly structured, difficult to test, maintain, and evolve. The transform method was proposed in 1983 by Balzer et al. to overcome this difficulty. In the transform method, all later modifications to code are made only to the specification, and not to the design, implementation, and testing stages. However, this method requires that one can convert formal specification of software into code that specifies the requirements. This method is effective in reducing the amount of software development time, and cost.

Then Boehm (1988) proposed the spiral method that evolved from the application of the above mentioned software development methods, especially the waterfall method. The proposition of the spiral method was an important contribution to software development because it was the first lifecycle method that explicitly took a risk-driven approach to software development, in contrast to the document driven, and the code driven approaches of the previous methods (Boehm, 1988). The spiral method takes an 
iterative approach to software development in contrast to sequentially approaching the phases of software development as was done previously. In this method, the lifecycle is presented in the form of a spiral, wherein the inception of software development is shown at the epicenter of the spiral and proceeds iteratively through every cycle of the spiral as software development progresses.

The above mentioned software development methods (including that of Boehm's) were heavily process based, with heavy emphasis on documentation. The limitations of these methods were realized by many software practitioners and were criticized by them. Some of the perceived limitations of these methods include their difficulty to learn and use them quickly, some of them are labor intensive, they are time consuming and thus slow down the development process, many of them are poorly defined and ambiguous to implement in practical situations (Tolvanen, 1998). Some of the other perceived limitations include the ability of the projects to adapt to the changing circumstances in the organizations and their projects, changing customer requirements. These limitations make them more restrictive and bureaucratic in their behavior (Kalermo and Rissanen, 2002).

The agile philosophy came into being to overcome some of the above challenges so as to become more usable in modern day dynamic organizations. The primary shift from the traditional software development practices to the agile ones involved laying lesser emphasis on process and documentation and paying more emphasis on quickly developing products and satisfying customers by incorporating their changing needs. Unlike the previous practices, the agile practices do not focus on plan-driven development. Further details about the usefulness of the agile methods will be discussed 
in the sections that follow. Table 4.1 in Chapter 4 discusses the major differences between the traditional plan-driven and the agile practices ${ }^{5}$.

\subsection{History and Evolution of Agile Software Development}

A group of seventeen software practitioners, who have been following some light-weight software-development methods, met in Snowbird, Utah, USA in 2001 to rationalize their common philosophy, and termed their shared philosophy of software development as "agile". They developed the Manifesto for Agile Software Development, which states the values and principles of the ASD philosophy. The Agile Manifesto is explained in Section 2.3.

\subsection{Agile Manifesto}

The Manifesto for Agile Software Development (Fowler and Highsmith, 2001; Fowler, 2002) is described below:

We are uncovering better ways of developing software by doing it and helping others do it. Through this work we have come to value:

- Individuals and interactions over processes and tools

- Working software over comprehensive documentation

- Customer collaboration over contract negotiation

- Responding to change over following a plan

That is, while there is value in the items on the right, we value the items on the left more.

(Agile Manifesto)

\footnotetext{
${ }^{5}$ We do not focus on any particular methodology within these practices. In this Thesis, we focus on the philosophical viewpoints advocated in these practices.
} 


\subsection{Principles of Agile Software Development}

The agile methodologies are based on a set of principles, which guide the generic nature of all the different agile methodologies that are proposed. Instead of evaluating whether the stated principle are right or wrong, which can, in fact be situation-dependent, in this Section we explain the principles behind agile software development.

The twelve principles stated in the Agile Manifesto are formally written below ${ }^{6}$ :

1. Highest priority is given to satisfying the customer through early and continuous delivery of valuable software.

2. Changing requirements are welcome, even late in development. Agile processes harness change for the customer's competitive advantage.

3. Working software is delivered frequently, from a couple of weeks to a couple of months, with a preference to the shorter timescale.

4. Business people and developers work together daily throughout the project.

5. Projects are built around motivated individuals. They are given the environment and support they need, and trusted to get the job done.

6. It is believed that the most efficient and effective method of conveying information to and within a development team is face-to-face conversation.

7. It is believed that working software is the primary measure of progress.

\footnotetext{
${ }^{6}$ Taken from http://www.agilemanifesto.org/principles.html
} 
8. It is believed that agile processes promote sustainable development. The sponsors, developers, and users should be able to maintain a constant pace indefinitely.

9. It is believed that continuous attention to technical excellence and good design enhances agility.

10. It is believed that simplicity--the art of maximizing the amount of work not done-is essential.

11. It is believed that the best architectures, requirements, and designs emerge from self-organizing teams.

12. It is believed that success is achieved when at regular intervals the team reflects on how to become more effective, then tunes and adjusts its behavior accordingly.

Elaborate discussions about the agile principles can be found in a number of pieces of literature such as Abrahamson et al. (2002), Ambler (2005c), Cockburn (2001), Cockburn (2002a), Cockburn (2002b), Fowler and Highsmith (2001), Fowler (2002), Highsmith (2004), and Schwaber (2004). We synthesize below some of the important discussions available in these pieces of literature on the agile principles.

One of the important principles behind ASD is giving high importance to customers. The agile practices aim to satisfy customers by producing valuable pieces of the final product early in the development lifecycle than handing in a finished product towards the end of the contract, as is normally done by traditional software development practices. As the customers are concerned about the working software, the ASD practices do not give 
importance to artifacts such as requirements and design drafts. The ASD practices emphasize fast and early delivery of pieces of software incrementally in shorter timescales.

To adapt with the volatility with today's businesses, the ASD practices also aim at continuously accepting changing requirements even late in the development. This is a significant difference with the traditional software development practices wherein the requirements are frozen towards the initial stages of the software development lifecycle. The ASD practices, on the other hand, are targeted to cope with the turbulences and uncertainties that typically accompany modern-day dynamic business environments.

The proponents of ASD believe that unlike purchasing tangible commodities, software cannot be ordered with a list of functionalities. Both the software developers and the business people need to work with each other to be able to come up with the final required product. Software development projects are normally very dynamic - in modern-day projects, requirements change, designs change, blocks of code, test processes change, and functionalities change due to valid reasons as development progresses. Therefore, it is important for the business, and the development teams to work hand-inhand throughout the entire duration of the project.

Due to the requirements of high agility proposed by the agile practitioners, the nature of communication plays an important role in the success of the agile practice being employed. Face-to-face communication is found to be effective in achieving this. The most popular form of communication in traditional development projects is documentation. However, documentation is often detrimental to agility. Furthermore, 
documentation increases the development overhead, which the agile practices aim to reduce. The reader may recall that the "agilists" advocate the production of those artifacts that will demonstrate working software to the customers.

ASD practices give importance to development around motivated individuals, give them the freedom and flexibility to make them productive, and "think outside of the box". The agile philosophy believes in project implementation around motivated individuals. Motivated individuals should be provided the environment, and support needed for them to make them successful.

Agile philosophers advocate that central to customer satisfaction is the development of working software. In other words, agile philosophers lay more importance on developing those artifacts that will directly lead to the development of the end product the working software. Project progress and performance measurement should be centered around the development of working software.

The agile philosophy believes in sustainable development, i.e., working with a constant pace, instead of working irresponsibly affecting the health of the developers. For instance, the agile philosophy discourages the development teams from working on a particular issue overtime, burning down the efficiency of the team members, and decreasing their future productivity. Instead, they should work a constant number of hours every week.

Agile philosophy pays equal importance to speed, flexibility, and quality of designs. They believe that agility is enhanced by good-quality designs. For example, the agile 
methodology, XP, emphasizes on refactoring for evolving the quality of designs with iterations, and time.

One of the important requirements of agile practices is to infuse simplicity in development. Using simple approaches in development increases the changeability of designs and processes. Agile methods encourage development to restrict to what is absolutely required. Minimizing the unnecessary overhead will help in keeping designs simple.

The agile practices encourage lean development using iterative, evolutionary approaches and self-organizing teams. Flexibility in the roles and responsibilities of the team members helps in innovation and creativity of the team members. Self-organizing teams also help in increasing the dynamics of interactions and communication between the team members. The agile methods do not give importance to following strict processes. There is no single methodology or process that can be applied to every situation. Artifacts developed using agile methods evolve, and the methodologies used are improved over time based on the local parameters in which a particular methodology is employed.

\subsection{Criticisms of Agile Software Development}

While the agile philosophy has been embraced since its inception by lot of software professionals, there have been criticisms from different corners of the software development community. The acceptance of the agile software development principles in industries (especially the large private, government or bureaucratic organizations) has been greatly influenced by how well the agile principles have been proven to have 
succeeded in the previous projects. Because of the infancy ${ }^{7}$ of the agile philosophy, concrete evidences $^{8}$ in favor of agile are relatively few. Many projects ${ }^{9}$ are gradually trying to use the agile methods in their development project and are reporting success stories using them. We feel, considering the age of the agile paradigm in software development, the number of success stories reported using it has been considerable. These success stories are mostly based on the practitioners' experiences and their perceptions. Our literature search does not reveal any concrete failure stories using agile $^{10}$. However, there have been some criticisms about the usefulness of this philosophy. We summarize below some of those criticisms ${ }^{11}$ reported in the literature.

1. Agile is over-hyped and misunderstood (Irons, 2006)

2. It is difficult to get the right people involved (Irons, 2006)

3. There is a danger of inappropriate application (Irons, 2006)

4. It can reduce quality through lack of rigor (Irons, 2006)

5. There is limited support for distributed development environments (Mahanti, 2006; Turke et al., 2002)

6. There is limited support for subcontracting (Turke et al., 2002)

\footnotetext{
${ }^{7}$ It should be noted that agile software development has become popular primarily from the beginning of the $21^{\text {st }}$ century and (arguably) it has the promise of transforming the software development activities in the future.

${ }^{8}$ For example, Reifer et al. (2002), in a very small-scale preliminary study involving 14 organizations, reports of organizations that had productivity improvement, cost reduction, time-to-market compression using the agile methods.

${ }^{9}$ Agile Alliance maintains a library of articles on agile, many of which report people's experiences using agile methodologies.

${ }^{10}$ However, it should be noted that although there are no failure stories reported, people have reported limitations and "lessons learned" using agile.
} 
7. There is limited support for building reusable artifacts (Mahanti 2006; Turke $e t$ al., 2002)

8. There is limited support for development involving large teams (Mahanti 2006; Turke et al., 2002)

9. There is limited support for developing safety-critical software (Mahanti 2006; Turke et al., 2002)

10. There is limited support for developing large, complex software (Turke et al., 2002)

11. Requires significant cultural change for adoption in projects (Stephens and Rosenberg, 2003)

12. Will work only when senior development team members are involved (Stephens and Rosenberg, 2003).

13. The amount of software design that is done may be insufficient (Stephens and Rosenberg, 2003).

14. Contractual negotiations may be difficult to attain (Stephens and Rosenberg, 2003).

15. Does not work for development projects for safety critical and reliable systems (Lindvall et al., 2002).

11 The criticisms 11-14 are particularly attributed to XP, which is one of the most popular agile methodologies. 
16. Will work only when the performance requirements are explicitly stated at the outset of the projects, and suitable test plans are planned (Lindvall et al., 2002).

17. Will work the best only for applications that can be built quickly without involving much complexity (Lindvall et al., 2002).

However, we feel, that most of the criticisms listed above are debatable, and there is insufficient data in the literature on support of these claims. Most of the unsupported claims of criticisms are taken from disparate patches of literature mentioning them.

Having said the above, we should clarify that our goal is not to establish whether ASD works in practice or not. Our goal is to identify the success factors from the perspective of software practitioners in ASD projects, identify the important changes required and the challenges that can be potentially encountered by projects willing to adopt ASD practices in projects. 


\section{Chapter 3 \\ Success Factors of Agile Software Development Projects}

In this Chapter we review the potential factors that affect the success ${ }^{12}$ of projects that want to adopt ASD practices. We review the parameters that affect the success of projects adopting ASD practices. The objective is to finally uncover empirical evidence in support of or against the above.

First we propose a theoretical framework (a priori framework) supporting the research objective on the basis of the previously published literature on agile software development. Thereafter, we formulate a set of hypotheses and validate them by collecting data from ASD practitioners in different industries. The later two are detailed in Chapter 5 and Chapter 6.

\subsection{Agile Software Development Project Success}

There are different works available in the literature that discuss the success of any IS activity (Delone and McLean, 1992; Delone and McLean, 2003). Delone and McLean's framework of IS success (Delone and McLean, 1992; Delone and McLean, 2003) is considered to be one of the most pioneering, popular, and breakthrough amongst the IS professionals. At the heart of their success framework are the considerations of use and user satisfaction as indicators of system and information quality. Use and user satisfaction, in turn, determine the individual impact and the organizational impact. 
Many other studies on measuring success are available, but we strongly believe that user or customer satisfaction ${ }^{13}$ is the ultimate goal of any IS activity, because all the other criteria that can be come up with would finally lead to determining the satisfaction of the users or the customers.

While there can be a list of different factors that can be identified, the holistic view of what project managers consider success in any software development project is worth considering. From a project manager's viewpoint, time, cost, and quality (Net Worth Consulting, 2004; PMI, 2004; Schwaber and Beedle, 2002) are three key components of any project's success. These are competitive criteria for software development project managers. Any disciplined software development activity has the primary goals of developing high quality software, with less time, and cost. We believe that these are three key attributes characterizing the success of any software development project, and most other success characteristics can be transformed into any of them. For example, productivity, customer satisfaction, and functionality can be perceived as quality criteria.

The challenging task for any software development project manager is balancing these three success criteria - time, cost, and quality. As shown in Figure 3.1, the position of "O" in the triangle signifies the cost, time, and quality impact in a software development project. For instance, greater quality of a software product can be achieved if there is greater budget and time at disposal. But project managers are normally constrained with

\footnotetext{
${ }^{12}$ In this document, at certain places we have used the term "agile software development success" to mean the success factors from the perspective of software practitioners in ASD projects.

${ }^{13}$ Here we assume that the customer is the user of the system.
} 
respect to both the time and budget they are allowed to work with, and the quality of the software the project is expected to deliver.

The same expectations are still valid for projects using agile practices. The reader should recall that the whole notion of agility in software development is "uncovering better ways of developing software" (Fowler and Highsmith, 2001; Fowler, 2002). In other words, any project using agile practices would be considered to have succeeded if it can deliver better quality software in shorter "timeboxes", and reduced budget than the traditional software development practices.

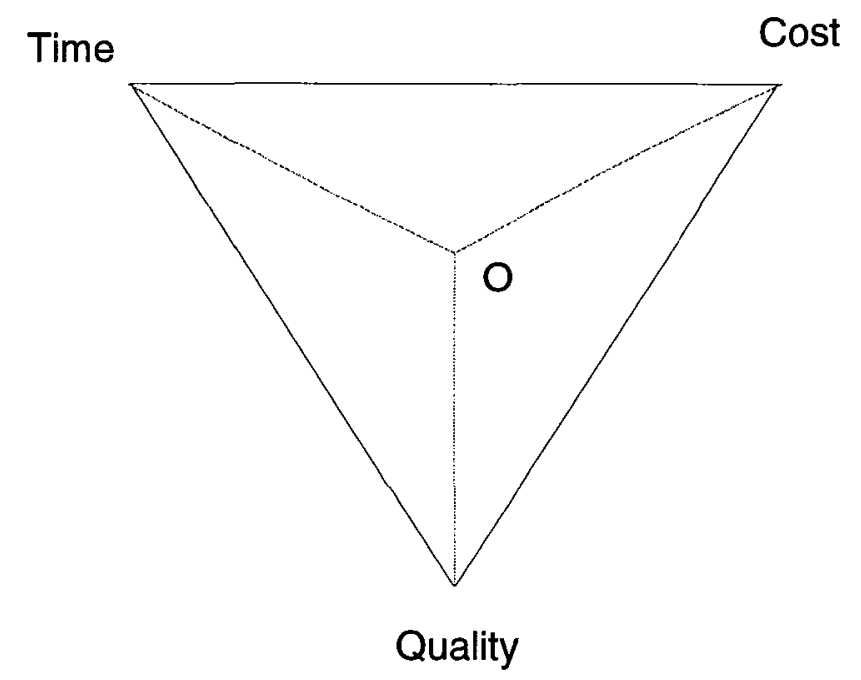

Figure 3.1: Quality/Time/Cost Tradeoff Triangle in Software Development

Jim Highsmith, in his popular book, "Agile Project Management" lists five business objectives of exploration processes, i.e., processes that are capable of operating in uncertain environments. They are listed below (Highsmith, 2004): 
1. Continuous innovation: This requires delivering products and services according to current customer needs and requirements. These needs are requirements that do not stem out of structured, plan driven environments. Continuous innovation is supported by adaptive organizational culture involving self-organization and self-discipline. It is measured by how a project can deliver customer value today.

2. Product adaptability: This requires delivering products according to changing customer requirements. These changes may happen within the duration of few weeks to few years. The product should be adaptable to the changing requirements. Ideally, such changes should be efficient and cost-effective.

3. Reduced delivery schedules: This requires reducing delivery schedules to meet the market requirements. Reducing delivery schedule should be accompanied by increasing return on investment (ROI). Careful attention is given to delivering primarily those features that are important to the customer. Marginally beneficial features are considered secondary.

4. People and process adaptability: This requires being dynamic with the changes in the business and the product. Similar to the adaptability of product, the people and the process need to adapt to the changes in the time-varying nature of the market. Instead of viewing changes with a resistive attitude, they should be made part and parcel of the businesses.

5. Reliable results: Traditionally, good traditional/plan-driven (predictable) processes required delivering products using processes having repeatable 
outcome, i.e., processes that would deliver the same even when undertaken after a lapse of time. These processes resulted in predictable outcome within the specified time and budget. However, for exploration processes, what is important is whether the project was able to deliver a valuable product to the customer within the specified time and budget. In exploration processes, although the outcome is not repeatable, innovative results are delivered to the customers in line with their vision.

Other pieces of published literature reveal the following expected quality criteria from agile software development projects:

1. Improved customer/business satisfaction (Opperthauser, 2003; Shine Technologies, 2003).

2. Increased flexibility to meet client's special needs (Opperthauser, 2003).

3. Improved business processes (Opperthauser, 2003).

4. Improved productivity (Shine Technologies, 2003).

5. Cost reduction (Reifer, 2002)

A good quality system can either possess perceived integrity or conceptual integrity (Poppendieck and Poppendieck, 2003). A system is said to possess perceived integrity if the product is holistically considered to be usable, reliable, operational, and economical. On the other hand, it is said to possess conceptual integrity if all the core functions and concepts work together smoothly. Whether a system has perceived integrity or conceptual 
integrity, what is important is that the customer should perceive the system to be costeffective and easily usable (Poppendieck and Poppendieck, 2003).

Based on the discussions of the different determinants of success in Section 3.1, we feel that they can be broadly classified to have the following three dimensions:

(i) Reduction of delivery schedules,

(ii) Increase in the return of investment (ROI) and the decrease in the costs.

- There are different models of ROI calculation (El Emam, 2004). The most simplified and the commonly used model in practice is as follows (Nucleus Research, 2002):

$$
\mathrm{ROI}=\frac{\text { Average benefit over a time period }}{\text { initial cost }} .
$$

These benefits can be tangible or intangible. Increased return on investment, therefore, is directly proportional to the increase in the average tangible or intangible benefits with respect to the initial investments made.

(iii) Improvement of quality. The improvement of quality criterion can have multiple dimensions. We consider the following attributes to represent the quality dimension:

(a) Ability to meet with the current customer requirements (continuous innovation)

(b) Flexibility to meet with the changing customer requirements (product adaptability) 


\section{(c) Improved business processes}

(a) and (b) above primarily determine the criteria for the satisfaction of the customers. In other words, to satisfy the customer it should be ensured that all the present and future requirements of the customers are met in time and within budget. In this work, we have intentionally opted to have (a) and (b) as separate criteria for customer satisfaction (instead of having a single criteria determining it), to understand if and how much the agile methods perform better in projects having changing requirements, which is one of the important promises of the agile philosophy.

This also aligns with the balanced scorecard methodology ${ }^{14}$, which views an organization from learning and growth, business process, financial, and customer perspectives and measures an organizations success accordingly. Here we are assuming that the success of an organization is partially dependent on the success of the software development projects.

To summarize, the following five criteria will be used to determine the success of (agile) software development (projects). All the other determinants of success discussed above are either logically included in any of the following criteria, or are considered relatively less important for the purpose of this research. These criteria will form the dependent variable in our research. They capture the three important dimensions of the success of any project, reduced time, reduced cost, increased quality, as mentioned

\footnotetext{
14 The balanced scorecard methodology aligns with some of the ideas of Total Quality Management (TQM) such as continuous improvement, customer-defined quality and measurement-based management (http://www.balancedscorecard.org/basics/bsc1.html).
} 
earlier. The above discussions provide the rationale behind the choice of these five dependent variables.

- $\quad$ Reduced delivery schedules

- Increased return on investment (ROI)

- Increased ability to meet with the current customer requirements

- Increased flexibility to meet with the changing customer requirements

- Improved business processes

\subsection{Success Factors}

\subsubsection{Organizational Factors}

\section{Customer Centric Issues}

Some of the authors who have advocated/cited customer centric issues as a factor are Cohn and Ford, 2003; Lindvall et al., 2002; McMahon, 2004; Nerur et al., 2005; Opperthauser, 2003; Turner and Boehm, 2003. The three types of customer centric issues they have considered can be broadly classified as:

- Customer Satisfaction

- Customer Collaboration

- Customer Commitment

The Agile Manifesto (Fowler and Highsmith, 2001) advocates customer collaboration as one of the important requirements for successful software development. One of the principles of ASD is giving highest priority to achieving customer satisfaction through early and continuous delivery of valuable software (Fowler and Highsmith, 2001). This requires that the customers are not only available on site with the software development 
team, but also highly motivated, active, and consider themselves responsible elements in the project. Customer commitment is, thus, an important success factor.

Close collaboration with customers provides the customers to closely involve/engage themselves into the development process, and also have a better opportunity of participating in some of the important business decisions taken inside the organization in regards to the project (Lindvall et al., 2002). For the development teams as well, having customers closely collaborate with them gives them an opportunity to adhere to changes in customer requirements faster without having to go through a formal approval processes for the changes. Of course, the above depends on the size and nature of the changes as well. Thus, in a project following the agile approach the customers and the developers have a better partnership opportunity, and finally, deliver a high quality product.

Unlike other products, software has different characteristics. It cannot be purchased as material items such as groceries. In software development, to be able to get a good quality product that would satisfy the customers, the process of outlining the requirements, setting up the deadline, and specifying the budget will not fetch a good product. It is also typical in software development projects that the requirements, budget, and the delivery time change as the customers and the development team interact more. Additionally, as customer requirements can be infeasible or difficult to meet as sought, it is also suggested that the customers and developers work together to understand the requirements, issues, and the feasible and infeasible items well. Without properly doing so, a software development project will be prone to failure.

Assertion: Customer satisfaction is important for the success of agile software development projects. 
Assertion: Customer collaboration is important for the success of agile software development projects.

Assertion: Customer commitment is important for the success of agile software development projects.

\section{Decision Time}

In the words of Ken Auer, “Agile is about taking control of your own destiny to the point that you can" (eWorkshop, 2002). Successful agile software development teams are normally left on their own, thereby allowing them to take their own decisions and succeed (eWorkshop, 2002). Also, as close customer, business area and software developer collaborations are advocated by agile practices (Fowler and Highsmith, 2001), important project decisions are likely to be made within a short timeframe. Agile practices are perceived to likely succeed in environments where rapid communication is enabled (eWorkshop, 2002). Rapid communication is likely to cut down the amount of time expended on major decisions. Therefore, fast decision-making is prominently an enabler of agility.

Assertion: Taking quick decisions is important for the success of agile software development projects.

\section{Team Distribution}

- Having centralized teams is also a likely factor positively affecting the success of projects. According to Ken Schwaber (eWorkshop, 2002), co-located teams are one 
of the important vehicles for successful communication, which is, in turn, identified by Scott Ambler (eWorkshop, 2002) as one of the important success factors of software development. Companies involved in distributed international projects will be affected by the cultural, and political situations in those regions. The geographic distribution and the location of the teams is important because the local political, cultural, and behavioral habits and situations greatly influence the productivity of the project team. For instance, in regions of the world where people are used to working collectively, agile approach will likely to be more successful in organizations that are located there than in organizations located in regions where that is not the case. Turner and Boehm (2003) have also advocated the team distribution factor.

- Further, the far off geographic distribution of teams may make communication, especially face-to-face communication, extremely difficult, thereby slowing down the pace of the projects.

Assertion: Team distribution is an important concern for the success of agile software development projects.

\section{Team Size}

The number of members in a team greatly influences the degree of communication that can be had between team members. For instance, in a team that has a large number of members, it is quite likely that frequent, informal, and rapid interactions will become difficult. Whereas the pace of informal communications can be made faster in small 
teams. Based on the literature (Dyba, 2000; eWorkshop, $2002^{15}$ ), we hypothesize a team with maximum 20-40 people would be optimum for good for agility. Having a larger team might pose great hindrance to fast communication and decision making in projects. However, if there are more people in a project, it is suggested that large teams be broken down into few smaller teams. Further, with the growth of the team size, efficiently coordinating/managing the interactions between people might also pose to be an important issue to be taken care of. The issue of team size has also been cited by Boehm and Turner (2003) as one of the factors.

Assertion: Appropriate team size is important for the success of agile software development projects.

\section{Corporate Culture}

"To be agile is a cultural thing. If the culture is not right, then the organization cannot be agile" (Lindvall et al., 2002). Having the right corporate culture is almost unanimously perceived by agile experts to be a necessary factor determining the introduction of agile practices (eWorkshop, 2002; Lindvall et al., 2002). Since agile practices emphasize "individuals and interactions over processes and tools", and "customer collaboration over contract negotiation" (Agile Manifesto), intuitively the nature of organizations individuals work in is important. For example, agile is not appropriate in bureaucratic organizations (eWorkshop, 2002).

\footnotetext{
${ }^{15}$ In this reference different team sizes are suggested, but we accept the suggestion of 20-40 members made by Alistair Cockburn, which was agreed by the majority of the participants.
} 
A dynamic, and fast changing organization will find agile methods extremely suitable for it (Abrahamson et al., 2002). Since the agile practices pay emphasis on customer collaboration and feedback, the organizational culture should be adaptive to changes. The organizational culture should also be supportive of working in a collaborative environment. Organizations that want to adapt agile practices need to have the culture of rapid communication, trusting people, supporting the decisions of the developers, encouraging changing requirements, and vehicles for fast feedback from customers (Lindvall et al., 2002).

In a bureaucratic organization, in which there are strict procedural requirements for making changes or introducing new ideas, agile will clearly be inappropriate in such an organization. Also, such organizations have the culture for proving the success of a methodology, tool, or process before it can be accepted by the management. This becomes resistive to introducing innovation in the organizations, and also increasing the pace of development that agile practices aim for. The more deeply rooted such a culture is in the organization, the more resistive the organizations will be to introducing and efficiently using the agile methodologies (Highsmith, 2000).

Some of the other authors who have advocated/cited corporate culture as a factor are Ambler, 2005c; Boehm and Turner, 2003; Cockburn and Highsmith, 2001; Cohn and Ford, 2003, Nerur et al., 2005 and Turner and Boehm, 2003.

Based on the previous discussions, we want to investigate whether the following aspects of corporate culture will have any implication on the success of agile software development projects: 
- Culture for rapid communication

- Culture for trusting people.

- Culture for supporting the decisions of the developers.

- Customer centric culture.

- Culture for encouraging fast feedback from customers.

- Culture for encouraging changing requirements.

- Non-bureaucratic management culture.

- Culture where the management, developers, and testers are in total agreement to use agile processes.

Assertion: Supportive corporate culture is important for the success of agile software development projects.

\section{Planning \& Control}

One of the important aspects that characterize the implementation of agile software development practices is the nature of organizational, management, and project planning and control. For instance, documented plans, accompanied by quantitative performance measures are considered key to the success of organizations practicing traditional/plandriven software development. On the contrary, internalized plans, and qualitative control are considered to succeed organizations adopting agile practices (Boehm and Turner, 2003). Some of the other authors who give importance to planning and control as a factor are Cockburn, 2002; Cohn and Ford, 2003; McMahon, 2004. 
Assertion: Internalized plans are important for the success of agile software development projects.

Assertion: Qualitative control is important for the success of agile software development projects.

\subsubsection{People Factors}

The success of a software development project depends on the how people and human resource factors. Evidently, this becomes more so in agile software development. This is because in agile practices, there is emphasis on individuals and interactions, customer collaboration, and responding to changes suggested by customers. We list below some of the important people factors.

Some of the factors mentioned below could equally be classified as organizational factors. However, instead of getting bogged down into the unimportant issue (according to the goals of this Thesis) of which category they belong to, we simply describe them here.

\section{Competency}

Competency means whether one has real-world experience in the technology domain, has built similar systems in the past, and possesses good interpersonal \& communication skills (Lindvall et al., 2002).

In the context of talent hunt, the following principle of Barry Boehm should be kept in mind "use better, and fewer people" (Cohn and Ford, 2003; Keith, 2002). Since the agile practices emphasize delivering working software fast, evidently, the competency of the 
team members in a project is important. From the discussions in Lindvall et al. (2002), it can be inferred that having at least $25 \%$ technically competent people in the projects is important. Previous experience in developing alike software as the current one is important in a project. The competency of the team members dictate not only the pace of development of a software, but also delivering the software according to the requirements of the customers.

Some of the other authors who talk about competency as a factor are Boehm and Turner, 2005 and Cockburn and Highsmith, 2001.

Assertion: Having at least $25 \%$ competent team members is important for the success of agile software development projects.

\section{Personal characteristics}

People factor plays a key role in the success of agile methods. According to Alistair Cockburn, "Good people are key to success with big teams" (eWorkshop, 2002). Ken Auer believes that having high quality people does not necessarily mean having experienced ones (eWorkshop, 2002). Not only the experience and competency of the team members is important, but also their personal characteristics such as honesty, collaborative attitude, sense of responsibility, readiness to learn, and work with others are considered equally important, if not more (eWorkshop, 2002).

Agile philosophers believe that just professional competencies are not enough anymore for a programmer. Agile principle's emphasis on the criticality of communication has indirect effects on the requirements of team members' qualities - without communication 
abilities, and desire to work in an agile team, even an expert may become an hindrance to the success of the project.

Thus having "good people" in the team, rather than following specific principles and practices, is considered important for the success if agile methods (Lindvall et al., 2002).

Some of the other authors who talk about personal characteristics as a factor are Ambler, 2005c; Boehm and Turner, 2003; Boehm and Turner, 2005; Cockburn and Highsmith, 2001 and Cockburn, 2002.

Assertion: Personal characteristics of the team members is an important for the success of agile software development projects.

\section{Communication and Negotiation}

Communication plays an important role in the implementation of agile practices in a software development project (Turner and Boehm, 2003). Effective communication is identified as one of the important factors for agile practices to succeed (Ambler, 2005b). According to Scott Ambler (eWorkshop, 2002), both processes and organization need to have in place a vehicle for communication. One of the important requirements for agility is fast and effective communication between developers, operations, support, customers, management, and business areas.

There are different modes of communication - paper-based, e-mail, telephone, video, and face-to-face (Ambler, 2005b; Cockburn, 2001). According to Cockburn (2001), person-to-person and face-to-face communication modes, accompanied by white-boards, flip-charts, and index-cards are the most effective means of communication for agile 
software development. Face-to-face communication is also identified as an efficient and effective mode of communication in one of the twelve principles of agile software development. However, the degree to which a particular mode of communication is effective over another is situational. The following are some of the factors affecting communication (Ambler, 2005b):

- Physical Proximity: Physical proximity, i.e., how close people are to one another, affects the level of communication. Close physical proximity may not necessarily mean having two or more people working together side-by-side. Two people working together in different floors of the same building, or two nearby buildings may also be considered to have close physical proximity.

- Temporal Proximity: This determines whether two or more people are working in the same (or similar) time-zones. This affects the way people can communicate with each other. In the current era of outsourcing, when many North American jobs are outsourced to Asian and European countries, temporal proximity has become an important issue. Asian and European outsourcing partners often end up matching their hours of work with their North American clients, who are in the opposite time-zones.

- Amicability: According to Cockburn (2001), amicability between people communicating with one another is an important factor affecting communication. This is because if people communicating with one another can trust each other, and speak with good will, greater flow of quality information 
can take place. Ambler (2005b) believes that the concept of everyone learning from everyone else is a critical success factor for effective communication.

Both physical and temporal proximity often enhances "osmotic communication", i.e., subconscious flow of information through overhearing of conversations between people, seeing things happening around them, and so on (Ambler, 2005b).

All the above forms of communication enhance the ability of the people to negotiate between themselves, by continuously talking and learning from each other. Communication and negotiation are, thus, by definition, important factors for the success of software development adopting the agile approach (eWorkshop, 2002).

Some of the other authors who have advocated the importance of communication as a factor are Boehm and Turner, 2003; Cockburn, 2001; McMahon, 2004 and Turner and Boehm, 2003.

Assertion: Communication and negotiation play important roles in the success of agile software development projects.

\section{Societal Culture}

Software development is similar to the development of other products in that the culture of the society in which the organization operates is important. As any other human activity, software development is highly influenced by regional (local) culture. The Agile Manifesto (Fowler, 2002) lays emphasis on individuals and interactions between people over processes and tools. The agile principles (Fowler and Highsmith, 2001) require motivated individuals. People should be eager to learn from each other, be honest, 
collaborative, and responsible (eWorkshop, 2002). All these are affected by the societal cultural factors as well. For instance, if a society has communicative people, if they are dynamic and progressive by their nature this will affect their work habits as well. Since we have emphasized earlier that personal characteristics are important success factors, so would be the societal culture, because the personal characteristics of the people are greatly influenced by their societal culture and vice versa.

Assertion: Societal culture plays an important role for the success of agile software development projects.

\section{Training and Learning}

An important concern that has been raised in the previous literature is how much training is required for successfully implementing agile practices in an organization (Lindvall $e t$ al., 2002). According to Bil Kleb, “"continued learning' is one of the fundamental differences between 'agile' and 'others'" (eWorkshop, 2002). People should be eager to share information with one other, continuously learn. This increases the chances of agile practices. The eWorkshop on agile (eWorkshop, 2002) had discussions around training of people - how important is training in agile methods, whether formal training is needed or not, and how much important training is in agile? The eWorkshop participants asserted that agile practices require less formal training (especially in the case of XP, because of pair programming), mentoring, and professionally guided discussions were more useful in agile than formal training, so that tacit knowledge could be transferred between individuals. According to Ken Auer (eWorkshop, 2002), training gets much easier if the people in the project are honest and collaborative. 
Some of the other authors who talk about training and learning as a factor are Boehm and Turner, 2005 and Drobka et al., 2004.

Assertion: Having team members willing to continuously learn and train each other is important for the success of agile software development projects.

\subsubsection{Summary of Independent Variables}

To summarize from the discussions in the above Section, all the above factors and their respective advocates or the authors who have cited these factors are listed in Table $3.1^{16}$. These factors will serve as the independent variables. A summary of them is listed as follows:

- Customer Satisfaction

- Customer Collaboration

- Customer Commitment

- Decision Time

- Team Distribution

- Team Size

- Corporate Culture

- Internalized Planning

- Qualitative Control

\footnotetext{
${ }^{16}$ Some of the discussions in few of the papers listed in this table and in the previous discussions led us to believe that they indirectly advocate the corresponding factor. These few papers did not always directly cite the corresponding factor.
} 
- Competency

- Personal characteristics

- Communication and Negotiation

- Societal Culture

- Training and Learning

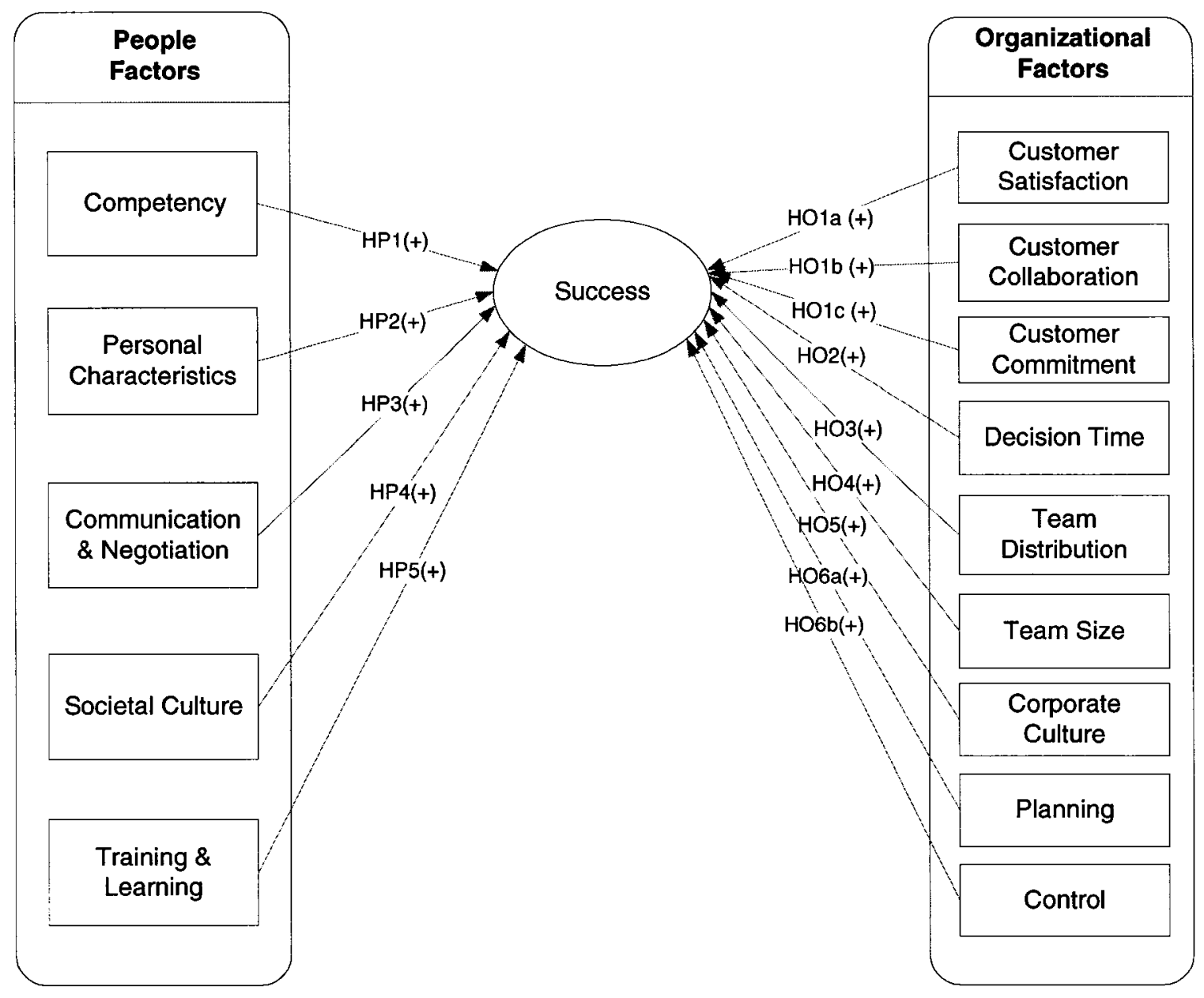

Figure 3.2: Theoretical Framework: Success Factors 
Table 3.1: A Summary of the success factors and their respective advocates.

\begin{tabular}{|c|c|c|}
\hline & Factor & Authors that have advocated/cited the factor \\
\hline 1 & Customer Centric Issues & $\begin{array}{l}\text { Cohn and Ford, 2003; Lindvall et al., 2002; } \\
\text { McMahon, 2004; Nerur } \text { et al., 2005; } \\
\text { Opperthauser, 2003; Turner and Boehm, } 2003\end{array}$ \\
\hline 2 & Decision Time & eWorkshop, 2002; Lindvall et al., 2002 \\
\hline 3 & Team Distribution & eWorkshop, 2002; Turner and Boehm, 2003 \\
\hline 4 & Team Size & Dyba, 2000; Boehm and Turner, 2003; \\
\hline 5 & Corporate Culture & $\begin{array}{l}\text { Abrahamson } \text { et al., 2002; Ambler, 2005c; } \\
\text { Boehm and Turner, 2003; Cockburn and } \\
\text { Highsmith, 2001; Cohn and Ford, 2003; } \\
\text { eWorkshop, 2002; Highsmith, 2000; Lindvall et } \\
\text { al., 2002; Nerur et al., 2005; Turner and Boehm, } \\
2003\end{array}$ \\
\hline 6 & Planning and Control & $\begin{array}{l}\text { Boehm and Turner, 2003; Cockburn, 2002; Cohn } \\
\text { and Ford, 2003; McMahon, 2004; }\end{array}$ \\
\hline 7 & Competency & $\begin{array}{l}\text { Boehm and Turner, 2005; Cockburn and } \\
\text { Highsmith, 2001; Cohn and Ford, 2003; } \\
\text { eWorkshop, 2002; Lindvall et al., 2002; Keith, } \\
\text { 2002; Turner and Boehm, } 2003\end{array}$ \\
\hline 8 & Personal Characteristics & $\begin{array}{l}\text { Ambler, 2005c; Boehm and Turner, 2003; } \\
\text { Boehm and Turner, 2005; Cockburn and } \\
\text { Highsmith, 2001; Cockburn, 2002; eWorkshop, } \\
\text { 2002; Fowler and Highsmith, 2001; Lindvall et } \\
\text { al., 2002; Nerur et al., 2005; Turner and Boehm, } \\
2003\end{array}$ \\
\hline 9 & $\begin{array}{l}\text { Communication and } \\
\text { Negotiation }\end{array}$ & $\begin{array}{l}\text { Ambler, 2005b; Boehm and Turner, 2003; } \\
\text { Cockburn, 2001; McMahon, 2004; Turner and } \\
\text { Boehm, } 2003\end{array}$ \\
\hline
\end{tabular}




\begin{tabular}{|l|l|l|}
\hline 10 & Societal Culture & $\begin{array}{l}\text { Cockburn and Highsmith, 2001; eWorkshop, } \\
2002 ; \text { Lindvall } \text { et al., 2002 }\end{array}$ \\
\hline 11 & Training and Learning & $\begin{array}{l}\text { Boehm and Turner, 2005; Drobka } \text { et al., 2004; } \\
\text { eWorkshop, 2002; Lindvall } \text { et al.., 2002 }\end{array}$ \\
\hline
\end{tabular}

\subsection{Research Hypotheses for Identifying the Success Factors}

On the basis of the theoretical framework developed from the previous literature, the following research hypotheses are formulated. These hypotheses are written as assertions in rectangular shaped boxes in Section 3.1. These assertions are based on evidences from the literature cited in the descriptions of the different success factors in Section 3.1. The truth of the hypotheses will then be validated using empirical data.

HO1a: The greater the satisfaction of the customers in projects, the more likely would be the success of agile software development projects.

HO1b: The greater the collaboration of the customers in projects, the more likely would be the success of agile software development projects.

HO1c: The greater the commitment of the customers in projects, the more likely would be the success of agile software development projects.

HO2: The quicker the decisions are taken in projects, the more likely would be the success of agile software development projects.

HO3: The more closely located the project teams are, the more likely would be the success of agile software development projects. 
HO4: The smaller the size of the teams in a project, the more likely would be the success of agile software development projects.

HO5: The stronger the corporate culture for agile software development projects, the more likely would be their success.

HO6a: The more informalized the plans are in an agile project, the more likely would be the success of agile software development projects.

HO6b: The more qualitative controls the projects have, the more likely would be the success of agile software development projects.

HP1: The more technically competent the team members are in a project, the more likely would be the success of agile software development projects.

HP2: The better the personal characteristics of the team members in a project, the more likely would be the success of agile software development projects. [Note: Some of the important personal characteristics for the success of agile projects include honesty, collaborative attitude, sense of responsibility, readiness to learn, and work with others].

HP3: The more the communication and negotiation is between people, the more likely would be the success of agile software development projects.

HP4: The more favorable the societal culture is for agile projects, the more likely would be the success of agile software development projects. [Note: The word "favorable" here refers to the individuals in the society being communicative, dynamic, progressive, and of similar societal culture]. 
HP5: The more the environment is for continuously learning, and informal training, the more evident would be the success of agile software development projects. 


\section{Chapter 4}

\section{Changes and Challenges/Risks in Adopting Agile Practices in Traditional Software Development Projects}

Most of the changes and challenges identified in the existing literature that are required for adopting agile processes in a traditional development projects are either based on experience of the developers, or are based on the opinions of agile experts without much empirical support. Additionally different previous research papers report long lists of such changes and challenges. However, for a project considering the adoption of agile practices, such long lists are of limited help. In this part of the research, we take a surveybased approach for obtaining empirical evidences of changes/challenges that need to be focused on while adopting agile processes. The list of changes/challenges we have come up with is not new to our work. Our contribution is something different. Specifically, we are interested in the statistical ranking of the changes/challenges that will be of help to project managers wanting to adopt agile software development practices.

In contrast to Chapter 3, where we were interested in identifying the factors responsible for the success of agile software development, in this Chapter we are interested in identifying the critical changes/challenges responsible for the transition of traditional software development projects into agile. This is an exploratory study. The objective is to determine possible candidates that are likely to affect the transition, and then rank them according to their importance, based on empirical evidences. As the list of changes required can be long and formidable for use, the idea is to help the transition managers in 
traditional projects by providing them with a ranked list of items (both changes and challenges/risks), which they can use to focus on the activities of primary importance.

\subsection{Home-Ground Characteristics: Rationale for Change}

Before discussing further about what changes are required and what challenges would be involved for transforming projects practicing traditional approaches into agile ones, let us first look at some of the home-ground characteristics of both of these development approaches. This will help us further to understand what changes in these home-ground characteristics the projects that want to adopt agile approaches would need to make.

A theoretical analysis of the propositions of both the agile approaches and the traditional disciplined ones was already done by Boehm and Turner (2003). According to them, no one methodology can be regarded as the best. Each of them has its own home ground, where it works better than the other - this should be, obviously, intuitive to most people, and should not be a surprise to anyone, because in reality it is hard to find some approach which can be regarded as a "silver bullet". Boehm and Turner proposed that instead of adopting just one methodology, balanced approaches dealing with a mix of both the agile and plan driven practices would be successful in the future. While agile approaches are better in dealing with issues relating to customer satisfaction, lower defect rates, faster changeability of requirements, and faster development times, the traditional/plan-driven approaches are better in ascertaining the predictability, stability, and high-assurance in the development processes. Their findings are summarized in Table 4.1. However, they have not cited any reference providing empirical support for their statements - their propositions seem to be experiential in nature. 
Table 4.1: Home grounds of agile and traditional/plan-driven methods [taken from Boehm and Turner (2003)]

\begin{tabular}{|c|c|c|}
\hline Characteristics & Agile & Plan-driven \\
\hline \multicolumn{3}{|l|}{ Application } \\
\hline Primary Goals & $\begin{array}{l}\text { Rapid value; responding to } \\
\text { change. }\end{array}$ & $\begin{array}{l}\text { Predictability, stability, } \\
\text { high assurance. }\end{array}$ \\
\hline Size & Smaller teams and projects. & Larger teams and projects. \\
\hline Environment & $\begin{array}{l}\text { Turbulent; high change; } \\
\text { project-focused }\end{array}$ & $\begin{array}{l}\text { Stable; low-change; } \\
\text { project/organization } \\
\text { focused. }\end{array}$ \\
\hline \multicolumn{3}{|l|}{ Management } \\
\hline Customer Relations & $\begin{array}{l}\text { Dedicated on-site } \\
\text { customers; focused on } \\
\text { prioritized increments }\end{array}$ & $\begin{array}{l}\text { As-needed customer } \\
\text { interactions; focused on } \\
\text { contract provisions. }\end{array}$ \\
\hline Planning and Control & $\begin{array}{l}\text { Internalized plans; } \\
\text { qualitative control. }\end{array}$ & $\begin{array}{l}\text { Documented plans, } \\
\text { quantitative control. }\end{array}$ \\
\hline Communications & $\begin{array}{l}\text { Tacit interpersonal } \\
\text { knowledge. }\end{array}$ & $\begin{array}{l}\text { Explicit documented } \\
\text { knowledge. }\end{array}$ \\
\hline \multicolumn{3}{|l|}{ Technical } \\
\hline Requirements & $\begin{array}{l}\text { Prioritized informal stories } \\
\text { and test cases; undergoing } \\
\text { unforeseeable change. }\end{array}$ & $\begin{array}{lr}\begin{array}{l}\text { Formalized } \\
\text { capability, }\end{array} \text { inteject } \\
\text { quality, } \\
\text { evolution requirements. }\end{array}$ \\
\hline Development & $\begin{array}{l}\text { Simple design; short } \\
\text { increments; refactoring } \\
\text { assumed inexpensive. }\end{array}$ & $\begin{array}{l}\text { Extensive design; longer } \\
\text { increments; refactoring } \\
\text { assumed expensive. }\end{array}$ \\
\hline Test & $\begin{array}{l}\text { Executable test cases define } \\
\text { requirements and testing. }\end{array}$ & $\begin{array}{l}\text { Documented test plans and } \\
\text { procedures. }\end{array}$ \\
\hline \multicolumn{3}{|l|}{ Personnel } \\
\hline Customers & $\begin{array}{l}\text { Dedicated, collocated } \\
\text { "CRACK" (Collaborative, } \\
\text { Representative, Authorized, } \\
\text { Committed, } \\
\text { Knowledgeable) }\end{array}$ & $\begin{array}{l}\text { "CRACK" performers not } \\
\text { always collocated. }\end{array}$ \\
\hline
\end{tabular}




\begin{tabular}{|l|l|l|}
\hline & performers. & \\
\hline Developers & $\begin{array}{l}\text { At least 30\% full-time } \\
\text { Cockburn* Level 2 and 3 } \\
\text { experts; no Level 1B or }-1 \\
\text { personnel. }\end{array}$ & $\begin{array}{l}\text { 50\% Cockburn* Level 3s } \\
\text { early; 10\% throughout; } \\
30 \% \text { Level 1Bs workable; } \\
\text { no Level -1s. }\end{array}$ \\
\hline Culture & $\begin{array}{l}\text { Comfort and empowerment } \\
\text { via many degrees of } \\
\text { freedom (thriving on chaos). }\end{array}$ & $\begin{array}{l}\text { Comfort and empowerment } \\
\text { via framework of policies } \\
\text { and procedures (thriving on } \\
\text { order). }\end{array}$ \\
\hline
\end{tabular}

* Cockburn levels of software method understanding and use:

Level 3: Able to revise a method (break its rules) to fit an unprecedented new situation.

Level 2: Able to tailor a method to fit a precedented new situation.

Level 1A: With training, able to perform discretionary method steps (e.g., sizing stories to fit increments, composing patterns, compounding refactoring, and complex COTS integration). With experience can become Level 2.

Level 1B: With training, able to perform procedural method steps (e.g., coding a simple method, simple refactoring, following coding standards and $\mathrm{CM}$ procedures, running tests). With experience can master some Level 1A skills.

Level -1: May have technical skills, but unable or unwilling to collaborate or follow shared methods.

We believe, Boehm and Turner's statement is easily said than done. Given the substantially opposite philosophies of the two approaches (e.g., emphasis on welldocumented plans in traditional approaches versus paying less importance on plans in agile approaches), it would be useful to stick to one type of philosophy in its most part to avoid confusions in the adoption of a particular type of philosophy over another during the course of the project. For traditional development projects intending to transform into agile, what should be instead done is to identify the most important changes required (instead of changing them all), and then focus on only those that are critical for the success of projects. Similarly, it would be useful to identify the critical challenges that such a transforming project would face. 
Irrespective of whether a project would like to transform in whole or in part to agile, in any transformation project, it is a common practice to focus on only the key areas at first and then gradually move attention to the other areas. To be able to do that, identifying the key changes required and the challenges involved for adopting agile philosophies in a project would be useful.

\subsection{Changes and Challenges}

In this Section, we present our work that may be helpful for software projects in the future while transforming projects that follow traditional software development processes into agile methods. Key to the work is the identification of the different key changes that will be required, and the challenges that need to be undertaken for adopting agile methods in a traditional software development project. A pictorial depiction of this is shown in Figure 4.1, and they are described in further detail in the latter part of this section. The different changes and challenges are identified based on some of the important existing literature discussing such issues. In this Thesis, we do not focus on the technical and application level changes. To avoid clumsiness of the Figure 4.1, we have listed these key identified changes and challenges in Figure 4.2. The native attribute types in Figure 4.2 are based on the home grounds of Boehm and Turner (2003), and the major challenge categories identified by Nerur et al. (2005).

In Figure 4.2, we present the list of changes that are required for adopting agile practices in traditional development projects, and the associated challenges/risks that are likely to affect such changes. As can be seen from this Figure, the challenges/risks have a negative relationship with the required changes, thereby indicating that the former are 
likely candidates to affect the transformation of traditional development processes into agile ones. The list ${ }^{17}$ of changes and challenges has been prepared based on the previous literature.

What we have tried to list in Figure 4.2 is all possible change candidates, and the risk factors. What would be interesting to know is what are the critical changes required and the risk factors. These will be empirically identified. As can be seen in Figure 4.2 the list of the changes and the challenges/risks is large. The intention is to obtain a list of empirically ranked changes, and challenges/risks that transformation managers can use to focus their attention on.

${ }^{17}$ In our list, we have tried to identify most of the important items. However, as agile software development is an emerging practice, our literature review reveals inadequate understanding of practitioners regarding all the different factors that are likely to affect the adoption of agile methodologies. Thus, we do not claim that the list is exhaustive. We, however, believe that we have been able to identify some of the important ones appearing in the literature. 


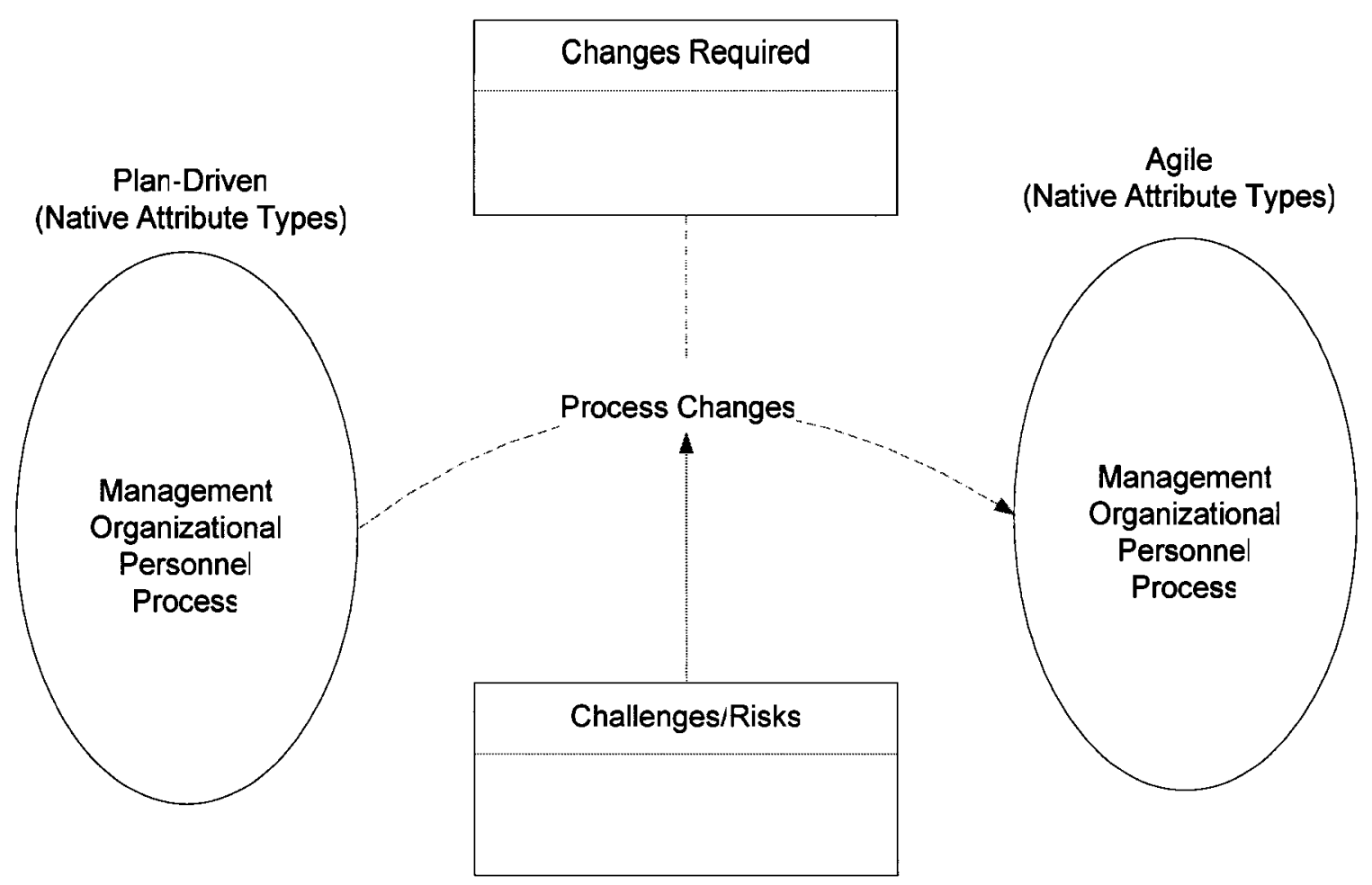

Figure 4.1: Transitioning Traditional Software Development Processes into Agile. 


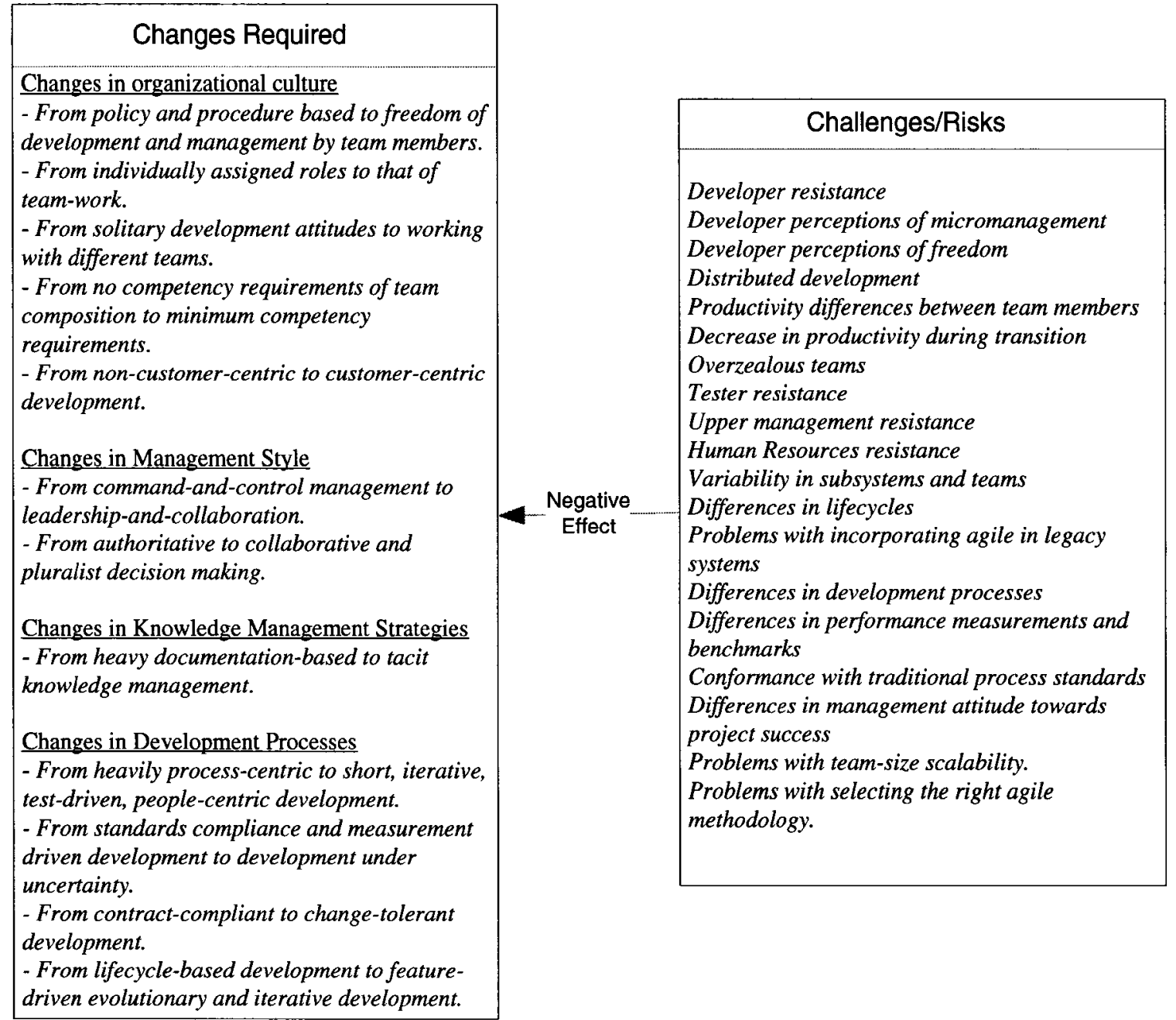

Figure 4.2: The changes required for adopting agile practices in traditional projects, and the associated challenges/risks.

There are a plenty of pieces of literature that talk about the changes required and the challenges involved in the transformation of traditional projects into agile - Boehm and Turner (2005), Cohn and Ford (2003), McMahon (2005), and Nerur et al. (2005) are a few recent important ones, to name a few. However, without elaborating the list of such authors, we have identified below some of the changes and challenges, which have 
gained a common consensus among different researchers and practitioners. Following the practice that is usual in similar kinds of Business/Information Systems research, initially we attempted to classify all the changes and challenges, as we did in Figure 4.2, and then tabulate the list of authors who have advocated/cited the change/challenge required (i.e., constructing a table similar to Table 3.1). However, after considerable investigation of all the relevant pieces of literature, we realized that the construction of such a table of citations is less important, and perhaps, irrelevant here. This is because the entries in list of these changes/challenges are intuitive, and fundamental, and therefore, most agile software development researchers/practitioners would agree with the change/challenge, whether they cite them explicitly or not. In other words, it is not appropriate in this work to classify authors who advocate a change/challenge item from one who would not. It would be unjustified to categorize the change/challenge items based on their advocates. The list in Figure 4.2 is obtained after critically examining and appraising the works of the previous authors. We should emphasize that all the changes and the challenges discussed below are not our suggestions/contributions - they have been listed in the literature mentioned above. Our contribution is to obtain a ranking of the changes/challenges according to their level of importance perceived from survey data. This ranking will help the managers planning to adopt agile practices in the future to focus on only the most important items instead of getting buried under the heavy load of working with all of them during a planned transition. 


\subsubsection{Changes Required}

\section{Changes in organizational culture}

One of the most important classes of changes that is required is the change in organization culture. Organizational culture needs a change from policy and procedure based to that of freedom of development and management by team members. The incorporation of agile practices influences the values, norms, behavior and actions of people, the decision making processes, problem resolution strategies, customary practices, and the relationships between employees and managers (Nerur et al. 2005). The cultural changes are challenging to achieve, as these require the change in the habits and mindsets of the people with which they have worked over the years.

Many authors, e.g., Boehm and Turner (2005), and Nerur et al. (2005) have identified people issues to play an important role in the adoption of agile practices. In traditional organizations many developers are used to solitary programming activities. These development habits that these programmers in traditional organizations have inculcated over the years can stand in the way of the successful adoption of agile practices. Many agile practices are based on the concepts of pair programming, collaborative decision making, onsite customers, and shared learning. The success of all of these activities is dependent on the attitudes of people in the team towards one another. Nerur et al. (2005) propose that working effectively in a team, high level of competence, and the relationships with the customers in terms of the commitment, knowledge, proximity, trust, and respect and important people issues that organizations should be concerned while transforming the existing processes into the agile ones. 
Customer centric people issues are especially important in agile organizations, because most agile practices require the developers to work closely with the customers. Both the customers and developers are responsible for successful collaborative decision-making. As a result due to the involvement of so many people from different backgrounds and psychology decision making can be more difficult than in traditional organizations, where the project managers are primarily responsible for most of the decision-making activities (Nerur et al. 2005).

\section{Changes in Management Style}

The adoption of agile processes in traditional organizations require a change in management styles from command-and-control management to leadership-andcollaboration. In traditional development projects, the project managers' roles are that of someone who would chalk out a well defined and documented plan and would exercise and control them. However, because of the paradigm shift from traditional/plan-driven in traditional projects to depending on ad hoc plans in agile, the role of the project managers also requires a change to someone who would facilitate the overall development of the projects, work with the team members equally, collaborate with them and coordinate their activities to ensure a smooth completion of the projects. Therefore, as we see, the project managers who are keen on adopting agile approaches in their organizations would also need to change their management style.

\section{Changes in Knowledge Management Strategies}

Another important issue is a paradigm shift in knowledge management strategies from heavy documentation-based to that of the management of tacit knowledge that mostly 
resides within the agile developers. This is a consequence of the agile approaches paying less importance on documentation. If there is a lack of documentation, most of the knowledge resides with the developers. Consequently, how this tacit knowledge will be managed brings in a new focus area in agile projects.

\section{Changes in Development Processes}

One of the important differences between traditional development practices and the agile practices is that while the former is heavily process-based, the emphasis on processes is relatively less in the latter. Instead, the agile practices pay more importance to peoplecentric development.

Development processes need to change from heavily process-centric to short, iterative, test-driven, people-centric development, from standards compliance and measurement driven development to development under uncertainty, from contract-compliant to change-tolerant development, and from lifecycle-based development to feature-driven evolutionary and iterative development (Nerur et al., 2005). Most of these changes have also been supported, implicitly or explicitly, by other authors listed at the beginning of Section 4.2.

\subsubsection{Challenges Involved}

\section{Developer resistance}

Developers in traditional development projects are accustomed to heavyweight development processes including production of non-code artifacts such as design diagrams, and data models. Production of such artifacts, and their documentation is often considered an important milestone of success in traditional development. However, as 
agile practices pay more attention to the production of code artifacts, and as the project success is dependent on that, agile practices can often lead to frustration, and therefore face resistance by the developers habituated with the traditional development processes

\section{Developer perceptions of micromanagement}

In agile software development projects, the managers interact with the developers more closely, and frequently than they do in traditional development projects. This might sometimes be greeted with unease by the developers used to traditional development processes, where the managers meet with their employees mostly primarily during the periodic status meetings. Meeting with their managers often might wrongly signal the agile developers that their managers are chasing them for the upcoming or missed deadlines. Similarly, managers lacking competency in agile principles might often forget the fine line of difference between micromanagement, and collaborative decision making and close developer-manager interactions required by some agile practices.

\section{Developer perceptions of freedom}

As agile practices do not have heavy-weight processes similar to the traditional development processes, some developers, especially the inexperienced ones, might take agile practices to have given them freedom of not having a day-to-day work schedule based on a Gantt/Pert Chart.

\section{Distributed development}

Agile teams are expected to expedite the decision making process. This requires faster communication between development team members, managers, and customers. Agile teams often rely upon face-to-face communication than written documented e-mails, 
mails, and notes. This, in turn, requires that teams be collocated. Distributed development may decrease the pace of communication, and in turn slow down the decision making required in agile-based development.

Productivity differences between team members

Differences in productivity of the team members can often stand in the way of the success of agile teams, especially when they are working on software delivery related activities. While it is not expected that all members in a team be competent, the ratio between the number of competent, and non-competent people in a team is often critical to agile software development projects.

\section{Decrease in productivity during transition}

During the transition from traditional development processes to the agile practices, the productivity of the teams may be decreased due to the team members' inexperience with the new techniques and ways of operation.

Overzealous teams

Overzealousness might also pose to be a roadblock in some development teams due to the perceptions of agility and fast decision making characterizing agile practices. Overzealousness might sometimes lead to making decisions without any forethought.

\section{Tester resistance}

Unlike in traditional development, agile practices do not separate the coding and testing phases, and the latter is not done sequentially after the former is completed. The testers and developers are required to work very closely in agile software projects to do both 
testing and coding simultaneously in every iteration. However, simultaneous coding and testing is often not warmly greeted by traditional testers. They might get a feeling of micromanagement due to their close interactions with the development teams.

\section{Upper management resistance}

Upper management often resist moving to agile practices, as they become concerned about promising features to customers within a contracted deadline, tracking project progress, and integrating agile teams with non-agile teams in a project. The traditional managers often feel a loss of control in the absence of Gantt charts, project plans, and work-breakdown structures.

The managers in traditional projects adopting agile practices perceive that they will not be able to commit the deliverables within a specific time to the customers. The managers often feel that adopting agile practices would lead to imprecise deliverables and goals.

\section{Human Resources resistance}

The human resource representatives in traditional organizations are used to assigning fixed roles and responsibilities to employees and measuring their performance based on those fixed assignments. They have a very constrained view of timekeeping, roles, and responsibilities of employees. Concepts of pair programming, and work-sharing are often resisted by human resource.

As agility requires dynamism of the team members with respect to the above parameters, there could be human resource issues that need to be dealt with when trying to introduce agile processes in a traditional development organization. The human 
resource strategies in traditional organizations are often hard-lined with strict descriptions of what each individual can and cannot do. This can be a hindrance while implementing agile methods, which promote developers to "think outside the box". Consequently, agile developers often end up taking non-traditional approaches. Human Resource preparedness is important in organizations before employing agile practices.

For successful transitioning human resource teams should be educated of the need for agile practices.

\section{Variability in subsystems and teams}

Both agile development and traditional development teams developing software for the same system can end up developing a completely different set of artifacts, which might cause integration issues. In other words, if one team is working with traditional software development practices, and the other working with the agile software development practices, they can be significant problems in the system integration level. For example, the need for agile teams requiring refactoring their designs according to the changing customer requirements can cause conflicts with the traditional software development approach of freezing interface specifications in the early stages of software development.

\section{Differences in lifecycles}

Differences in lifecycles can also cause development process conflicts. While agile processes believe in test-driven design, short, and focused iterations of development, the traditional development processes are heavyweight, and have long iterations. Agile developers target delivering immediate value to the customers, whereas in traditional 
development the customers see the entire product towards the end of the development lifecycle.

Problems with incorporating agile in legacy systems

Agile development calls for prompt adaptiveness of systems to changing requirements. Thus, adopting agile processes in legacy systems could be a problem, because refactoring is inherently difficult in such systems.

\section{Differences in development processes}

While agile approaches tend to advocate the gathering of informal functional requirements, the traditional requirements processes are more formal, and they emphasize both functional and non-functional requirements such as reliability and security.

\section{Differences in performance measurements and benchmarks}

In traditional software development organizations, contract negotiation, and performance measurements are based on strict sequential milestone targets (with room for parallel development at times). This can cause problems while implementing agile practices, because agile developers go beyond the traditional code and design reviews by incorporating continuous customer feedback into the development lifecycle, and delivering functionalities in shorter timeboxes.

\section{Conformance with traditional process standards}

Many organizations have adopted process standards, e.g., CMM, CMMI, and ISO, in the past. Many of such organizations have even certified themselves to have attained certain process standardization levels. Although both CMM Level 5 (Optimized) and agile 
processes have some commonalities in terms of their philosophies of continuous improvement, and optimization of processes iteratively, they significantly differ in some of their fundamental philosophies. For example, the emphasis the traditional methods lay on documentation do not strictly conform with the agile philosophy.

Differences in attitude towards project success

Organizations often end up taking either of the two extreme steps towards success or failure of projects employing agile practices. The project managers are either fired or are promoted/rewarded for the project failures or successes. However, this type of organizational attitudes can pose as roadblocks to the successful implementation of agile processes in organizations.

\section{Problems with team-size scalability}

Agile approach fits better for projects with small team sizes. Team dimension influences communication between team members. The agile approach with face-to-face communication suites perfect for teams less than 20-40 people (Dyba, 2000). Therefore, traditional development projects with large teams transforming into agile will have its own challenges.

\section{Problems with selecting the right agile methodology}

According to Nerur et al. (2005), the other important process-centric issue of concern in the adoption of agile practices is the determination of the agile methodology of right-fit in a particular organization. Different organizations have different characteristics, and different agile methodologies differ with respect to different parameters such as team size, and the duration of the iterations. 


\section{Chapter 5 \\ Research Methodology}

As mentioned briefly in Chapter 1, the research methodologies we use in this Thesis are those typically used in survey-based business research. The core of the methodology is survey-based, post-facto investigation. Broadly it consists of the following three phases:

Phase I: Formulation of the research questions, construction of the theoretical frameworks and the development of hypotheses based on the research questions.

\section{Phase II: Collection of data.}

Phase III: Analysis of the data collected as part of Phase II.

We use the theoretical frameworks we have constructed in Chapters 3 and 4, and the hypotheses we have formulated, to design a questionnaire, which were pre-tested for its validity, usefulness, and readability before it was sent out for data gathering from the field. The data that were gathered were then statistically analyzed using suitable data analysis techniques explained in Chapter 6.

We use those techniques to gain an understanding of the important factors responsible for the success of projects that want to adopt agile practices, a ranked list of changes (according to their importance) that are necessary for transforming a project practicing traditional software development into an agile one, and the critical challenges/risks that the projects should be prepared to undertake. 


\subsection{Research Methodologies: Choices \& Rationale}

\subsubsection{Research Design}

Our research methodology closely relates to the scientific and empirical types ${ }^{18}$.

The two options that we considered to choose from in this methodology are: (i) experimental, or (ii) ex-post-facto, depending on whether the causal factors (or the independent variables) could be controlled or not.

In experimental studies involving cause-effect analyses, it is customary to investigate how one causal factor influences the dependent variable. To do so, normally, all the other possible causal factors, expect the one whose influence on the dependent variable is being investigated, is controlled. If, on the other hand, the researcher takes no action to control any of the independent variables whose effect on the dependent variable is being undertaken, the research design is called the ex-post-facto. As this study is based on the survey respondents' previous experience and perception using agile software development practices, our research design is of the ex-post-facto type.

\subsubsection{Data Source: Surveys}

We conducted a survey type research, where multiple software development projects practicing agile software development techniques were surveyed. We did not restrict our

\footnotetext{
${ }^{18}$ In the scientific method, a theory is initially developed in conjunction with a phenomenon to explain the latter, then a hypothesis is proposed, and different alternatives of the hypothesis are tested using data collected. The collected data may either support the hypothesis, or reject its claims. In the empirical method also, a hypothesis is developed. Then the hypothesis is validated using statistical mechanisms. Although the empirical method may sound similar to the scientific method, the difference between the two is that, unlike the latter, the former may not have a formal method or theory that can explain the hypothesis. In empirical
} 
surveys to any particular industry type, sector, or size. The only requirement we had was that the survey respondents practice agile software development, and they have transitioned in the past from traditional software development practices to those advocated by the "agile" software development community.

\subsubsection{Survey Technique}

The survey was administered using both the web-based and mail questionnaire techniques. The advantages of using these techniques stem out of the nature of the problem we investigate. Since we were interested in gathering the experiences of a large number of users of agile software development practices, we sent our survey questionnaire to potential respondents in various industrial sectors (such as manufacturing, electronics, telecommunications, aerospace, oil and gas) that are practicing agile software development techniques. Similarly, we did not restrict ourselves to particular organization/project size, culture, or nationality. With this approach we were able to survey agile software development professionals ${ }^{19}$ that are widely geographically distributed across continents, it was cost-efficient, and that the respondents were able to answer the questions more thoughtfully by taking their own time. Additionally, both the web-based and the mail questionnaires allowed the survey respondents to complete parts of the survey according to their own time. This also enabled them to do some "homework", if necessary, before being able to answer some of the questions.

method, the hypothesis is verified only using the collected data (Adrion 1993; Kontio 2001; Lazaro and Marcos. 2005; Zelkowitz and Wallace 1998).

${ }^{19}$ The eligibility of the respondents of the survey questionnaire is given in Section 5.1.2.3. 
For sending out the questionnaires by mail, as it was a challenge to find the exact postal addresses of respondents practicing agile software development, the addresses of the human resource departments were obtained from the company web-sites, and then they were approached to help us find the appropriate contact person(s) within their organization.

The mail questionnaire technique is reported in the literature to be intrinsically plagued by the limitation of very limited turnaround from the respondents (Clover and Balsley 1979; Creswell 2003; Newman 2005). In our study, we observed that we received all the responses through the web-based survey, and surprisingly, there were no returned filled-in questionnaires from those that were sent by post. The reason for this could be that the mailed questionnaires were sent to the general human resources departments of different organizations asking them to forward them to agile practitioners in their organizations who have practised plan-driven software development in the past. It would have likely happened that the human resource departments were not aware of exactly which individuals in their organizations would satisfy the eligibility criteria. It is also an undocumented fact that the human resource departments are generally more resistant to make the employees in their organizations overburdened with additional works like this. On the other hand, the web-based surveys were sent to the specific individuals and teams who work with agile methodologies. Secondly, in many cases the human resource departments were sent an e-mail with the same request as was sent by post. It might have happened in many cases that the human resource departments had forwarded the e-mails to the concerned people who filled out the web-based questionnaire and ignored the questionnaire they had received by post, as for many people filling out an web-based 
questionnaire is faster than sending by post. Finally, most of the questionnaires were sent outside Canada (within Canada only two organizations were approached with postage prepaid envelopes). Although a return envelope was enclosed with the questionnaires sent overseas, it was not possible to affix prepaid postage with the return envelopes that were sent out. This might have discouraged most respondents to pay for the postage and send it back.

\subsubsection{Questionnaire Design}

Respondents were asked to fill out a structured questionnaire (see Appendix B). The questionnaire has primarily close-ended, multiple choice type questions, with some openended questions at the end.

The advantage of the open-ended questions is that they enable the respondents to provide some additional information, which was not captured in the close-ended questions. These open ended questions were not used in statistical analysis, but their responses to such questions are summarized in Chapter 6. The multiple-choice type questions ask the respondents to rank their responses on a scale of 1 to 5 (Likert's scale). The close-ended multiple-choice type questions helped us to perform statistical analysis on the available data.

The nature of the questions helps to primarily assess the following three issues:

- The factors the respondents think will influence the success of projects that want to adopt agile software development projects. 
- The changes they think are important to transform a plan-driven software development project into an agile project.

- The challenges/risks they think will impact the transformation of a plan-driven software development project into an agile project.

The questionnaire also has classification type questions (e.g., occupation, and industry sector of the respondents) (Clover and Balsley 1979; Creswell 2003; Newman 2005). The classification type questions aid in gaining an understanding of the respondents' background $^{20}$.

\subsubsection{Identifying the Respondents}

In our research, identifying the respondents was a challenge, because agile software development is still an emerging approach that has gained popularity only in the last 4-5 years. In organizations, often only a few teams are practicing agile software development. Therefore, it was challenging to identify the specific respondents (and teams) in organizations who qualify to respond to our questionnaire.

We did considerable amount of research on this issue, and have been fortunate to find the following sources for collection of data:

\footnotetext{
${ }^{20}$ This was done in the following way. In the questionnaire, there is a section that collects the respondents' background information such as the primary business of the organization of the respondent, organization size, team size of the respondent, the respondent's role in the team, the number of years of knowledge of agile software development practices, and the number of years of experience developing software following agile practices. In the final analysis results, we provide a summary of the background of the respondents. This summary can help one to gain an overall understanding of the group of respondents whose consolidated responses were used in the data analysis.
} 
- CIO Magazine's August 2004 issue lists the awardee organizations of the "Agile 100 " awards. However, this list identifies only the candidate organizations on which we can focus. Determining the specific contacts in these organizations still remained a challenging issue.

- Agile Alliance (http://www.agilealliance.org) has a publicly available list of all its corporate members. As they are members of the Agile Alliance, it is likely that these organizations have teams who practice agile software development. We sent our questionnaires by post to these organizations.

- Agile Alliance was also requested to circulate the questionnaire to its individual members as they were not willing to reveal the contact e-mail addresses of those members. They agreed to help in this process.

- Previous research papers and experience reports published in this area by industrybased authors were collected. Those authors were approached by e-mail to fill out the web-based questionnaire.

- The contact person of different agile user groups throughout the world was contacted by e-mail to fill out the web-based questionnaire. They were also requested to forward the questionnaire to other members in their user group and also to the other team members at their place of work.

- The committee members of the different agile conferences that are currently announced or have already taken place in the past were also approached. 
- Several blog and website postings of the survey link were also done. The respondents were also requested to help in doing this in blogs they were associated with.

In cases where we knew the names of the organizations that potentially practice agile software development, but did not have the contacts of the specific teams which practice agile software development, we approached the human resources departments of those organizations and asked them to forward the questionnaires to the potentially eligible respondents within their organizations.

In almost all cases in which we were successful in obtaining the specific names of individuals who potentially practice agile, we sent out a reminder after a week or so to remind them, in case they had not already filled out the questionnaire.

In both the web-based and the mail questionnaire techniques, we did not keep track of the names (or similar other particulars) of the individuals who filled out the questionnaire. Of course, in the web-based survey that we had performed, the survey tool that we had used, i.e., Survey Monkey, keeps track of the IP addresses of the computers that were used to fill out the questionnaire and disallows multiple submissions from the same IP address.

The depth of data analysis that we could perform depended on the number of responses that we could obtain. Our initial target was to obtain at least 150 responses. Further discussions about the minimum sample size for our study are provided in Section 6.2.

The respondent eligibility criteria that were set to consider responses for further consideration of data analysis were the following: 
(1) Does the respondent's team practice at least six of the twelve ${ }^{21}$ agile software development principles?

(2) Has s/he ever been part of a team that used to practice traditional, plandriven software development, and had adopted agile practices in the past?

\subsubsection{Response Rate}

We received 241 responses, of which there were 174 were eligible responses, i.e., responses which satisfied the eligibility criteria mentioned above, for the success factors survey, and 165 for the changes survey, and 161 for the challenges survey, respectively. It was not possible to calculate the response rate because, in contrast to mail-in questionnaires in which a fixed number of survey questionnaires is printed and sent out to potential respondents, and the response rate is calculated based on the number of returned filled-in questionnaires, in web-based or e-mail surveys that use the methodology similar to the one in this Thesis it is difficult to keep track of actually how many people get to see and fill out the questionnaire.

In this context, it is also pertinent to mention that some of the agile experts (respondents) sent back messages encouraging and supporting this work. Excerpts of their responses are mentioned below. Their names are withheld in the interest of maintaining their anonymity.

\footnotetext{
${ }^{21}$ The rationale behind this is that it might be infeasible to find projects which follow all the 12 agile principles. This is because as agile software development was still an emerging philosophy, finding projects that follow agile practices is difficult, and finding projects that follow all the practices was even more difficult (if not, nearly impossible). Therefore, initially we set the target to identifying projects that follow at least $50 \%$ of those principles. While there was no hard rationale behind choosing this figure, we felt that this figure was neither too high nor too low.
} 
Respondent A: I think it's good for those of us in the agile community to support this type of research. The survey doesn't take long and it sounds like the results will be of interest.

Respondent B: This is one of the few surveys I have seen in this area that was not a total waste of my time. Congratulations to you and your committee.

Respondent C: I have more than ten year of experience in software development and five of this within an agile context. I've just completed your survey and think questions are very relevant. I would appreciate to receive the result of this work. You got a very good subject for your thesis.

Respondent D: ...My next book will be on scaling agile methods to large projects. Also, as you may know from my 'agile...' book and its "Evidence" chapter, I collect evidence, and write it up (which i'll do again in my next book). Therefore, I'm interested in any data from this survey you can share. Possible?

Respondent E: I am on the commercial side and so would be very interested in seeing your thesis when published. Do you have any objection to sending me a copy when complete?

In addition to the above experts, there were plenty of other respondents who showed interest in the final results from this work. This shows that, at least initially, a number of agile software development practitioners have "warmly greeted" the nature of this work, and have shown interest in it. 


\subsubsection{Pre-Testing the Questionnaire}

It was necessary to pre-test the questionnaire, before sending it out for the actual survey. Request for pre-testing was sent out to ten potentially qualified software development professionals. Out of the ten requests, only five agreed to help (50\% turnaround rate). Pre-testing was finally done with the help of those five software development professionals. They had different experience levels and work functions and worked in both the government and the private sectors. While four of the five respondents did not have any major suggestions for improvement, one suggested a few changes for improving the clarity of the questions. Overall, there were not much revision that was required as a result of the pre-testing.

Pre-testing the questionnaire was helpful for clarifying any ambiguities in the questionnaire, its readability, and the ordering of the questions. Based on the results of the pre-test, the original questionnaire was revised to reflect the feedback of the pre-test respondents. 


\section{Chapter 6 \\ Data Analysis}

After all the data were collected from the surveys, they were analyzed in an attempt to draw meaningful conclusions out of them. Different techniques for analyzing survey data were used. In this Thesis, the following procedures were undertaken:

1. Data Preparation: Here the main goal was to check the consistency of the raw data that was obtained, log them, edit them, code them, and thus, prepare them for analysis in the next step.

2. Data Analysis: The processed data from the previous step were then used with different techniques to "mine" information out of them.

3. Documentation: All the findings from previous two steps were then documented (which constitutes the bulk of the Thesis here). Apart from the fact that documentation in the form of this Thesis is a degree requirement, documentation is important because without proper documentation, the effort expended in the previous two steps could be wasted. However simple it may sound, we advocate this to be an important knowledge management step, because in the absence of this step all the results that are obtained, and the results of the analysis that are performed on them may not be beneficial to future researchers. 


\subsection{Data Preparation}

As part of data preparation, we mainly performed three main steps: data logging, data editing, and data entry. They are further explained below ${ }^{22}$.

\subsubsection{Data Logging}

Data logging was done to help us to keep track of the sent out, returned, edited, coded, and data entered questionnaires. However, simple it may sound, this was an important step because we adopted a variety of avenues for data collection (as mentioned in the Chapter 5), and there were a plenty of respondents that we had to communicate with. Without doing it in a structured, carefully planned, and organized manner it could have lead to cumbersomeness, and ambiguity later on, which could consequently lead to errors in data analysis.

In our research, we used Excel $^{23,24}$ to keep track of such information in an $a d$ hoc manner. There was no particular template that was used for this purpose. The different variables used in this study were coded (labeled) for ease in representation, and analysis using SPSS. The different codes that were used for each of the variables used for performing the studies are listed in Appendix C.

\footnotetext{
${ }^{22}$ The techniques we used are standard, and can be found in any business research method books such as Clover and Balsley 1979; Creswell 2003 and Newman 2005.

${ }^{23} \mathrm{http}: / / \mathrm{www}$.microsoft.com/

${ }^{24}$ This is in addition to the built-in data logging capabilities of the online survey tool "SurveyMonkey" (http://wwwsurveymonkey.com).
} 


\subsubsection{Editing}

The data that were collected were first edited before they were used for further analysis, to ensure that the data used for analysis reporting were accurate, uniform, legible, and consistent (Clover and Balsley 1979; Creswell 2003; Newman 2005).

During editing we checked for omissions to see whether all answers, and other associated data were recorded. Similarly, we checked whether the filled-in questionnaires returned by the respondents had any prominent inconsistent information. No prominently inconsistent filled-in questionnaires that were found. However, there were a few responses in which although a bulk of questions had been answered by the respondents, but there were a few missing entries. We decided not to remove those data points altogether from the main data sheet used in the data analysis. This was because, by doing so, we could end up losing information contained in the rest of the correctly filled out questionnaires. However, while doing some of the analysis using SPSS, SPSS by default ignores those points that have a missing value and performs the analysis only with the non-missing values ${ }^{25}$. We decided to go ahead with this strategy because by removing the data points with missing values altogether from the main data sheet, we would not be able to get even the descriptive statistical patterns underlying all the data.

Also, since errors might creep in due to misinterpretation or misunderstanding of the question on the part of the survey respondent, as mentioned in Chapter 5, we took precautionary measures by pre-testing the questionnaire. The respondents were also

\footnotetext{
${ }^{25}$ The default option of SPSS ignores those sample points that have even one missing value. The other option could have been to replace the missing value with the average of all the others. However, we adopted the default option, as
} 
provided our contact information to enable to contact us, in case they needed any clarification.

\subsubsection{Data Entry}

Since all the responses we obtained were through the web-based survey tool, "SurveyMonkey", we used the export capability of the tool to collect data obtained through the tool into Excel to enable their structured viewing, and manipulation. As a consequence, no separate manual entry of data was required. This, of course, minimized the possibilities of errors occurring during manual data entry. The Excel data sheet that is produced by the tool also automatically adds some additional columns such as the IP address of the computers that the respondents used for filling out the surveys. Those undesired columns were removed because they were not given any consideration during the further processing of the data. Later on during statistical analysis using SPSS, the Excel data sheet was imported as an SPSS data sheet to be able to perform statistical analysis using it in SPSS.

\subsection{Data Analysis}

The approach for data analysis was quantitative (statistical) in nature, with some stints of qualitative analysis of the data collected through replies to the open-ended questions in the questionnaire. The responses to the open-ended questions from the survey participants are listed in Appendix D.

we felt it was a fairer judgment to ignore such points instead of replacing them with the average (which could very easily be misrepresentative of what that respondent would have actually responded). 
We performed different statistical studies with the processed data of the closed-ended questions. They are listed below for each research question.

\subsubsection{Research Question 1}

As part of our first research question, on the basis of the survey data we obtained, we wanted to understand the success factors from the perspective of software practitioners in agile software development projects. It is a relationship study, and we have 14 independent variables (some of which have multiple questions measuring them) and a consolidated dependent variable ("Success"), which has 5 parts in our study ${ }^{26}$. In addition, we go one step further, and show the regression models with each of these constituents of Success as a dependent variable and the rest of the fourteen potential factors as the independent variables.

\subsubsection{Descriptive Statistics}

First we performed descriptive statistics to help us in summarizing the information latent in the data that we obtained from surveys for this research question. First, for each question corresponding to both the independent and the dependent variables, we tried to gain an understanding of the minimum, maximum, mean, and standard deviation of the survey data obtained from respondents. Table 6.1 shows the descriptive statistical results. It should be observed that, just by considering the mean values, amongst all the fourteen independent variables Ind 1 has the highest mean of 4.77 with a standard deviation of

\footnotetext{
${ }^{26}$ For variables that have multiple questions measuring them, for every respondent, we decided to average his/her responses over all the questions measuring it. Similarly, since "Success" has 5 measures, we decided to average out the responses over all the measures to obtain its consolidated measure.
} 
0.59. In other words, nearly most of the participants in this survey strongly agree that they give highest priority to customer satisfaction more than anything else. Ind2 (customer collaboration), Ind3 (customer commitment), Ind4 (decision time), Ind6 (team size), Ind7 (corporate culture), Ind10 (technical competency), Ind11 (personal characteristics), Ind12 (communication and negotiation), and Ind 14 (training and learning), according to the survey results were also considered to be in the range of (somewhat agree, strongly agree). The means of all the independent variables also show that Ind5 has the least mean value of 3.22. This shows that most of the participants remained neutral regarding whether most of the team members in their projects were geographically closely located, and the other teams that they interact with are geographically closely located. It should also be observed that none of the independent variables has a mean score below 3 ("Neither Agree nor Disagree" in the 5-point Likert scale that we used). This shows that on an average, the participants that were surveyed were not in disagreement with the factors in their projects.

It addition to the summarized descriptive statistical results presented in Table 6.1, the detailed descriptive statistical results with the individual variables constituting to form the consolidated independent variables are listed in Section E.1.1 in Appendix E. 
Table 6.1: Research Question 1: Summarized Descriptive Statistics

\begin{tabular}{|l|r|r|r|}
\hline \multicolumn{4}{|c|}{ Descriptive Statistics } \\
\hline \multicolumn{1}{|c|}{$\mathrm{N}$} & \multicolumn{1}{|c|}{ Mean } & Std. Deviation \\
\hline Ind 1 & 174 & 4.76 & .595 \\
Ind 2 & 174 & 4.17 & .925 \\
Ind 3 & 174 & 3.95 & 1.074 \\
Ind 4 & 173 & 4.30 & .815 \\
Ind 5 & 174 & 3.22 & 1.267 \\
Ind 6 & 174 & 4.64 & .899 \\
Ind 7 & 174 & 4.16 & .793 \\
Ind 8 & 174 & 3.70 & 1.227 \\
Ind 9 & 174 & 3.60 & 1.501 \\
Ind 10 & 173 & 4.11 & .985 \\
Ind 11 & 174 & 4.39 & .577 \\
Ind 12 & 173 & 4.02 & .748 \\
Ind 13 & 172 & 3.75 & 1.001 \\
Ind 14 & 171 & 4.34 & .783 \\
Dependent & 173 & 4.37 & .557 \\
Valid N (listwise) & 167 & & \\
\hline
\end{tabular}

Table 6.2 gives an overall idea of the different types of organizations to which the different respondents belong. It can be seen that the majority of the respondents (around $32.5 \%$ ) belong to industries whose primary line of business is computer related, followed by respondents who belong to consulting organizations (around $29.8 \%$ ). There were also around $10.2 \%$ responses from organizations having the primary line of business as banking/insurance industries, around $5.1 \%$ having the primary line of business as education/research, and around $2.4 \%$ each from medical/health organizations, and aerospace organizations.

In addition to the above organizations, there were about $7.9 \%$ respondents who belong to other organizations having primary line of business such as travel, logistics, transportation, government contractor, agriculture, and integrated workplace management. There were no responses from the hospitality, legal services, and non-profit 
organizations. Thus we can see that there has been a fair share of responses from different organization sectors.

Table 6.2: Primary identity of the respondents' organizations

\begin{tabular}{|l|l|l|}
\hline \multicolumn{1}{|c|}{ Identity } & Response percent \\
\hline 1 & $\begin{array}{l}\text { Computer related } \\
\text { (IS/MIS/DP/Hardware/Software/Telecommunications) }\end{array}$ & $32.5 \%$ \\
\hline 2 & Banking/insurance & $10.2 \%$ \\
\hline 3 & Real estate & $1.2 \%$ \\
\hline 4 & Business supplies/services & $1.2 \%$ \\
\hline 5 & Education/research & $5.1 \%$ \\
\hline 6 & Entertainment/media/publishing & $1.2 \%$ \\
\hline 7 & Hospitality & $0 \%$ \\
\hline 8 & Medical/health care & $2.4 \%$ \\
\hline 9 & Government & $2 \%$ \\
\hline 10 & Engineering/construction & $0.8 \%$ \\
\hline 11 & Consulting & $29.8 \%$ \\
\hline 12 & Legal services & $0 \%$ \\
\hline 13 & Manufacturing/distribution & $2 \%$ \\
\hline 14 & Consumer retail/wholesale & $0.8 \%$ \\
\hline 15 & Non-profit/membership organization & $0 \%$ \\
\hline 16 & Electrical machines & $0.4 \%$ \\
\hline 17 & Aerospace & $2.4 \%$ \\
\hline 18 & Others & $7.9 \%$ \\
\hline
\end{tabular}

The respondents were also requested to identify the number of employees in their organizations. Their responses are summarized in Table 6.3. Of all the respondents, roughly $27.6 \%$ belong to organizations having 501-1000 employees, roughly $26.8 \%$ having more than 1000 employees, roughly $11.8 \%$ having less than 10 employees, 
roughly $9.8 \%$ having $21-40$ employees, roughly $7.9 \%$ having $41-100$ employees, and roughly $5.9 \%$ having $10-20$ employees. Thus, we can see that the majority of the respondents belong to organizations having more than 500 employees. The number of responses from small sized organizations is also roughly around $27-28 \%$. Thus there has been fairly good response from both relatively small sized and large sized organizations.

Table 6.3: The number of employees in the respondents' organizations

\begin{tabular}{|l|l|l|}
\hline \multicolumn{1}{|c|}{ Number of employees } & Response percent \\
\hline 1 & Less than 10 & $11.8 \%$ \\
\hline 2 & $10-20$ & $5.9 \%$ \\
\hline 3 & $21-40$ & $9.8 \%$ \\
\hline 4 & $41-100$ & $7.9 \%$ \\
\hline 5 & $101-500$ & $10.2 \%$ \\
\hline 6 & $501-1000$ & $27.6 \%$ \\
\hline 7 & Greater than 1000 & $26.8 \%$ \\
\hline
\end{tabular}

Similarly, the respondents were also requested to identify the number of employees in their teams ${ }^{27}$. Their responses are summarized in Table 6.4. We see that most of the responses (roughly $33.3 \%$ ) have come from respondents belonging to teams having strength of 5-10 employees, roughly $26.3 \%$ in teams having 11-20 employees, and roughly $19.4 \%$ in teams having less than 5 employees. Around $10.3 \%$ responses each

\footnotetext{
${ }^{27}$ It should be noted that whether project size or organization size matters or not is not being investigated as part of this research. The reason such information have been collected is to get a statistic of the size of the organizations/projects of the respondents of the survey, along with the other statistics collected from the background information of in the survey questionnaire. These statistics would hopefully help us to get a better insight of the results from success factors and the changes/challenges study. Whether project size matters or not is a different research question altogether, which is worth investigating, but to draw firm and meaningful conclusions, one needs to do an in-depth study by varying the project size and framing the questions accordingly. While the value of such a work is appreciable, it will increase the length of the already long questionnaire. Similar comments apply regarding the effect of project complexity as well.
} 
were obtained from teams having 21-40 employees, and greater than 40 employees. Thus we can see that more than $50 \%$ of the respondents belong to small sized teams (having less than 10 employees). Roughly another $50 \%$ responses came from teams having more than 10 employees. This shows a fair distribution of participation from all sizes of teams.

Table 6.4: The number of employees in the respondents' team

\begin{tabular}{|l|l|l|}
\hline & \multicolumn{1}{|c|}{ Number of employees } & Response percent \\
\hline 1 & Less than 5 & $19.4 \%$ \\
\hline 2 & $5-10$ & $33.3 \%$ \\
\hline 3 & $11-20$ & $26.2 \%$ \\
\hline 4 & $21-40$ & $10.3 \%$ \\
\hline 5 & Greater than 40 & $10.3 \%$ \\
\hline
\end{tabular}

Table 6.5 shows the roles of the different respondents. Most of the responses (about 29.5\%) have been obtained from developers/testers, about $18.9 \%$ from team leaders, about $17.7 \%$ from project managers, and $7.9 \%$ from functional managers. There were also about $25 \%$ responses from respondents having other roles such as director, business analyst, software architect, scrum master, and usability analyst.

Table 6.5: The role in the respondents' team

\begin{tabular}{|l|l|l|}
\hline & \multicolumn{1}{|c|}{ Role } & Response percent \\
\hline 1 & Functional Manager & $7.9 \%$ \\
\hline 2 & Project Manager & $17.7 \%$ \\
\hline 3 & Team Leader & $18.9 \%$ \\
\hline 4 & Developer/Tester & $29.5 \%$ \\
\hline 5 & Other & $26 \%$ \\
\hline
\end{tabular}


The summary of responses of the duration for which the respondents have been developing software using agile principles/methods is depicted in Table 6.6. Majority of the respondents (roughly 30\%) have been developing software using agile principles/methods for $1-3$ years, $26.4 \%$ for $3-5$ years, $24 \%$ for greater than 5 years, and $19.6 \%$ for less than 1 year. This shows that we had participants from fairly all duration levels.

Table 6.6: Duration for which the respondents have been developing software using agile principles/methods

\begin{tabular}{|l|l|l|}
\hline & \multicolumn{1}{|c|}{ Duration } & Response percent \\
\hline 1 & Less than 1 year & $19.6 \%$ \\
\hline 2 & $1-3$ years & $30 \%$ \\
\hline 3 & $3-5$ years & $26.4 \%$ \\
\hline 4 & Greater than 5 years & $24 \%$ \\
\hline
\end{tabular}

In order to assess the degree to which the different respondents practice agile software development principles, the respondents were asked to mark their response in the 1 to 5 Likert scale ( 1 - Strongly Disagree, and 5 - Strongly Agree). Their responses are summarized in Table 6.7. The response average values imply that on an average the majority of the respondents agree with the statement that they practice most of the principles to a great extent (greater than 4). In fact, a majority of the respondents have stated that for all of 12 principles they strongly agree with the statement that they follow them. Gaining further understanding of the nature of distribution of the responses across all the scale items can be had by closely going through Table 6.7 . 
Table 6.7: Degree of practice of agile software development principles by the respondents.

The percentage values represent the percentage of the respondents who responded who selected that option in the 1 to 5 Likert scale.

\begin{tabular}{|c|c|c|c|c|c|c|c|}
\hline & $\begin{array}{c}\text { Strongly } \\
\text { Disagree } \\
\text { (1) }\end{array}$ & $\begin{array}{c}\text { Somewhat } \\
\text { Disagree } \\
\text { (2) }\end{array}$ & $\begin{array}{c}\text { Neither } \\
\text { Disagree } \\
\text { nor } \\
\text { Agree (3) }\end{array}$ & $\begin{array}{c}\text { Somewhat } \\
\text { Agree (4) }\end{array}$ & $\begin{array}{c}\text { Strongly } \\
\text { Agree } \\
\text { (5) }\end{array}$ & $\begin{array}{c}\text { Not } \\
\text { Applicable } \\
\text { or Don't } \\
\text { Know (X) }\end{array}$ & $\begin{array}{c}\text { Response } \\
\text { Average }\end{array}$ \\
\hline $\begin{array}{l}\text { We give high } \\
\text { priority to satisfying } \\
\text { customers through } \\
\text { early and continuous } \\
\text { delivery of valuable } \\
\text { software }\end{array}$ & $2 \%$ & $2 \%$ & $5 \%$ & $12 \%$ & $77 \%$ & $2 \%$ & 4.63 \\
\hline $\begin{array}{l}\text { We welcome } \\
\text { changing } \\
\text { requirements, even } \\
\text { late during } \\
\text { development }\end{array}$ & $3 \%$ & $7 \%$ & $9 \%$ & $37 \%$ & $42 \%$ & $2 \%$ & 4.11 \\
\hline $\begin{array}{l}\text { We deliver working } \\
\text { software more } \\
\text { frequently, from } \\
\text { couple of weeks to } \\
\text { couple of months, } \\
\text { with a preference to } \\
\text { a shorter timescale }\end{array}$ & $3 \%$ & $2 \%$ & $5 \%$ & $19 \%$ & $69 \%$ & $2 \%$ & 4.52 \\
\hline $\begin{array}{l}\text { Our business people } \\
\text { and developers work } \\
\text { together daily (very } \\
\text { closely) throughout } \\
\text { the project }\end{array}$ & $4 \%$ & $10 \%$ & $6 \%$ & $29 \%$ & $49 \%$ & $2 \%$ & 4.12 \\
\hline $\begin{array}{l}\text { We build projects } \\
\text { around motivated } \\
\text { individuals. We give } \\
\text { them the } \\
\text { environment and } \\
\text { trust them to get the } \\
\text { job done. }\end{array}$ & $2 \%$ & $6 \%$ & $8 \%$ & $31 \%$ & $51 \%$ & $2 \%$ & 4.24 \\
\hline $\begin{array}{l}\text { We emphasize more } \\
\text { on face-to-face } \\
\text { communication for } \\
\text { conveying } \\
\text { information to and } \\
\text { within the } \\
\text { development team. }\end{array}$ & $1 \%$ & $3 \%$ & $8 \%$ & $23 \%$ & $63 \%$ & $2 \%$ & 4.46 \\
\hline $\begin{array}{l}\text { We measure and } \\
\text { track progress based } \\
\text { on working software }\end{array}$ & $3 \%$ & $7 \%$ & $6 \%$ & $27 \%$ & $55 \%$ & $3 \%$ & 4.28 \\
\hline $\begin{array}{l}\text { We promote } \\
\text { sustainable } \\
\text { development, Our } \\
\text { sponsors, } \\
\text { developers, and } \\
\text { users maintain a }\end{array}$ & $5 \%$ & $10 \%$ & $11 \%$ & $32 \%$ & $39 \%$ & $3 \%$ & 3.93 \\
\hline
\end{tabular}




\begin{tabular}{|l|l|l|l|l|l|l|l|}
\hline $\begin{array}{l}\text { constant pace } \\
\text { indefinitely. }\end{array}$ & & & & & & & \\
\hline $\begin{array}{l}\text { Our software } \\
\text { development project } \\
\text { team follows } \\
\text { continuous attention } \\
\text { to technical } \\
\text { excellence and good } \\
\text { design for } \\
\text { development. }\end{array}$ & $4 \%$ & $5 \%$ & $7 \%$ & $39 \%$ & $43 \%$ & $2 \%$ & 4.14 \\
\hline $\begin{array}{l}\text { We practice simple } \\
\text { designs, processes, } \\
\text { and approaches in } \\
\text { our software } \\
\text { development } \\
\text { methodologies. We } \\
\text { implement features } \\
\text { that are required by } \\
\text { the customers- } \\
\text { nothing more. }\end{array}$ & $2 \%$ & $11 \%$ & $10 \%$ & $33 \%$ & $41 \%$ & $2 \%$ & 4.01 \\
\hline $\begin{array}{l}\text { Our development } \\
\text { teams are self- } \\
\text { organizing- our } \\
\text { teams can (re)- } \\
\text { organize } \\
\text { continuously in } \\
\text { different } \\
\text { configurations to } \\
\text { meet the changing } \\
\text { requirements and the } \\
\text { newly arising } \\
\text { challenges of the } \\
\text { business. }\end{array}$ & $5 \%$ & $12 \%$ & $14 \%$ & $33 \%$ & $33 \%$ & $3 \%$ & 3.79 \\
\hline $\begin{array}{l}\text { At regular intervals, } \\
\text { our team reflects on } \\
\text { how to become } \\
\text { more effective, then } \\
\text { tunes and adjusts its } \\
\text { behavior } \\
\text { accordingly. }\end{array}$ & $3 \%$ & $6 \%$ & $14 \%$ & $33 \%$ & $43 \%$ & $1 \%$ & 4.08 \\
\hline
\end{tabular}

In addition to the above summary of the respondents' backgrounds, the consolidated summary of the responses (in percentage figures) obtained from all the respondents for the different success factor variables is captured in Section F.1 of Appendix F. The results presented there are self-explanatory and are, thus, not explained further over here to maintain the brevity of this Chapter. However, three interesting observations are given below: 
- Amongst all the independent variables, Ind1 (i.e., customer satisfaction) was strongly agreed as being practiced by the largest (roughly 77\%) number of respondents.

- Amongst all the independent variables, the largest percentage (roughly 28\%) of respondents strongly disagreed that the other teams that they interacted with within or outside their organizations were geographically closely related to theirs (see the row corresponding to Ind5b in the table).

- Amongst the five items measuring dependent variable, "increased flexibility to meet with the changing customer requirements" was voted as being true by the largest percentage $(71 \%)$ of respondents.

\subsubsection{Test of Internal Consistency Reliability}

Our next step was to understand the internal consistency of items in a scale. This helps us to understand how consistent the different questions are within a variable or how consistently the survey respondents have responded to the items.

For testing internal consistency reliability, we used Cronbach's alpha ${ }^{28}$. Cronbach's alpha helps in estimating the proportion of systematic or consistent variance in a given sample of test scores. The value of Cronbach's alpha lies between 0 (no consistent variance can be accounted for) and 1 (variance is all consistent). A higher value of Cronbach's alpha indicates a greater consistency in variance of the sample test scores.

\footnotetext{
${ }^{28}$ There is another variation of the commonly used Cronbach's alpha, called the "Standardized Item Cronbach's Alpha". It is computed by standardizing the scale items so that they have equal means and variances. Some researchers use this value of alpha in their tests. In our study, we will present the results of both of these versions.
} 
Usually, a Cronbach's alpha value greater than 0.6 is considered standard in survey research. Some statisticians use 0.7 as the required cut-off point. A higher value of Cronbach's alpha would indicate a greater reliability or accuracy of the statistical inferences from the data. For example, if in a study the Cronbach's alpha for a set of test scores turns out to be 0.7 , it would mean that there is $70 \%$ consistency, and $100 \%-70 \%=$ $30 \%$ of inconsistency of test scores. Table 6.8 shows the Cronbach's alpha of those constructs that are measured with multiple items. As we can see the Cronbach's alpha for all items are at greater than or equal to 0.6 (in fact, they are mostly at least 0.7 ). This indicates that there are no problems with the internal consistency reliability tests. The inter-item correlation matrices corresponding to the items in Ind7, Ind11, Ind12, and Ind13 are given in Section E1.2 in Appendix E.

Table 6.8: Research Question 1: Reliability Statistics Summary

\begin{tabular}{|c|c|c|c|}
\hline Items in & Number of items & Chronbach's Alpha & $\begin{array}{c}\text { Chronbach's } \\
\text { Alpha Based on } \\
\text { Standardized } \\
\text { Items }\end{array}$ \\
\hline Ind7 & 8 & .872 & .878 \\
\hline Ind11 & 6 & .847 & .852 \\
\hline Ind12 & 5 & .700 & .664 \\
\hline Ind13 & 2 & .784 & .779 \\
\hline Dependent & 5 & .717 & .744 \\
\hline
\end{tabular}

\subsubsection{Linear Multiple Regression Analysis}

The purpose of this study is to understand how the 14 independent variables relate to the dependent variable (Success). Throughout the analysis, the dependent variable was log (base e)-transformed, as it was found that the log (base e)-transformed distribution gives a better linear regression model. The distribution obtained gets closer to normal. 
One of the outputs of running linear multiple regression analysis in SPSS is a table with the analysis of inter-correlations. Correlation analysis helps us to understand the degree of relationship between all our 14 independent variables and the dependent variable (Success). Table 6.9 shows the summarized correlation results, between the dependent variable and each of the independent variables, for items with non-missing values. The detailed inter-independent variable correlation results are presented in Appendix E.

Table 6.9: Research Question 1: Summarized Correlations Table

\begin{tabular}{|c|c|c|}
\hline Variables & $\begin{array}{c}\text { Correlation } \\
\text { coefficient }\end{array}$ & Significance \\
\hline Ind1 & .140 & .036 \\
\hline Ind2 & .232 & .001 \\
\hline Ind3 & .253 & .001 \\
\hline Ind4 & .254 & .000 \\
\hline Ind5 & .074 & .171 \\
\hline Ind6 & .097 & .107 \\
\hline Ind7 & .241 & .001 \\
\hline Ind8 & .075 & .168 \\
\hline Ind9 & .301 & .000 \\
\hline Ind10 & .102 & .096 \\
\hline Ind11 & .180 & .010 \\
\hline Ind12 & .104 & .092 \\
\hline Ind13 & .381 & .000 \\
\hline Ind14 & .283 & .000 \\
\hline$*$ N $=$ 166 & & \\
\hline
\end{tabular}

This analysis results in mainly two sets of values: (i) the Pearson's coefficient of correlation and (ii) the values of significance. The coefficient of correlation between a 107 
pair of variables indicates the degree of relationship between them. Its value lies between 0 and 1 . The higher the value of the correlation coefficient, the greater the strength of the relationship. A positive value of the coefficient indicates a positive relationship, whereas a negative value indicates an inverse relationship between those variables. The values of significance indicate the significance of each correlation coefficient in (i). The tests of significance are normally conducted at values 0.01 or 0.05 . In this study, the test was conducted at level 0.05 . If for a particular relationship, we find that the significance value is very small (less than 0.05 ), then it would indicate that the relationship is significant. If the relationship is between an independent variable and the dependent variable (Success), it indicates that, that particular independent variable has significant effect on Success. On the basis of the data that we have obtained we see that the independent variables Ind1, Ind2, Ind3, Ind4, Ind7, Ind9, Ind11, Ind13, and Ind14 are statistically significantly related to the dependent variable, Success, whereas the independent variables Ind5, Ind6, Ind8, Ind10, and Ind12 were found not to have any significant relationship with Success. In sum, the following factors are significantly related to Success:

\section{- Customer satisfaction}

\section{- Customer collaboration}

\section{- Customer commitment}

- Decision time

\section{- Corporate culture}

\section{- Control}




\section{- Personal characteristics}

\section{- Societal culture}

\section{- Training and learning}

The following are not significantly related with Success:

- Team distribution

- Team size

- Planning

- Technical competency

- Communication and negotiation

On the basis of the significance values and the values of correlation coefficients, we either accept or reject our 14 hypotheses we have listed in Chapter 3. The acceptance/rejection results of hypotheses mentioned in Chapter 3 are as shown below:

HO1a: The greater the satisfaction of the customers in projects, the more likely would be the success of agile software development projects. Accepted.

HO1b: The greater the collaboration of the customers in projects, the more likely would be the success of agile software development projects. Accepted.

HO1c: The greater the commitment of the customers in projects, the more likely would be the success of agile software development projects. Accepted.

HO2: The quicker the decisions are taken in projects, the more likely would be the success of agile software development projects. Accepted. 
HO3: The more closely located the project teams are, the more likely would be the success of agile software development projects. Not Accepted.

HO4: The smaller the size of the teams in a project, the more likely would be the success of agile software development projects. Not Accepted.

HO5: The stronger the corporate culture for agile software development projects, the more likely would be their success. Accepted.

HO6a: The more informalized the plans are in an agile project, the more likely would be the success of agile software development projects. Not Accepted.

HO6b: The more qualitative controls the projects have, the more likely would be the success of agile software development projects. Accepted.

HP1: The more technically competent the team members are in a project, the more likely would be the success of agile software development projects. Not Accepted.

HP2: The better the personal characteristics of the team members in a project, the more likely would be the success of agile software development projects. Accepted.

HP3: The more the communication and negotiation is between people, the more likely would be the success of agile software development projects. Not Accepted.

HP4: The more favorable the societal culture is for agile projects, the more likely would be the success of agile software development projects. Accepted.

HP5: The more the environment is for continuously learning, and informal training, the more evident would be the success of agile software development projects. Accepted. 
Another outcome of the multiple regression analysis that was conducted is a table containing the coefficients of the estimated regression model. It is shown in Table 6.10. These coefficients help us to construct a regression model showing the relationships between the dependent variable (Success) and the 14 independent variables, viz.:

$$
\text { Success }=\sum_{\mathrm{i} \in \mathrm{N}} \alpha_{\mathrm{i}} \mathrm{x}_{\mathrm{i}}
$$

In the above model, $\mathrm{N}$ represents the set of independent variables, and $\alpha_{i}$ and $\mathrm{x}_{\mathrm{i}}$ represent respectively the elements of the set of coefficients and the independent variables.

In our study, we perform stepwise linear multiple regression. In our stepwise regression study, the following criteria (default criteria in SPSS) are used in steps until these criteria are no longer satisfied:

Probability-of-F-to-enter $<=.050$, Probability-of-F-to-remove $>=.100$

Section E.1.3 in Appendix E shows the variables that are entered/removed in each step Each of these steps produces a model. Hence they are referred to be so to be consistent with the terminology used in SPSS. The Beta, t, Significance, and Collinearity Statistics for the excluded variables corresponding to each of these four steps are also presented in Section E.1.3 in Appendix E. 
Table 6.10: Research Question 1: Regression Coefficients

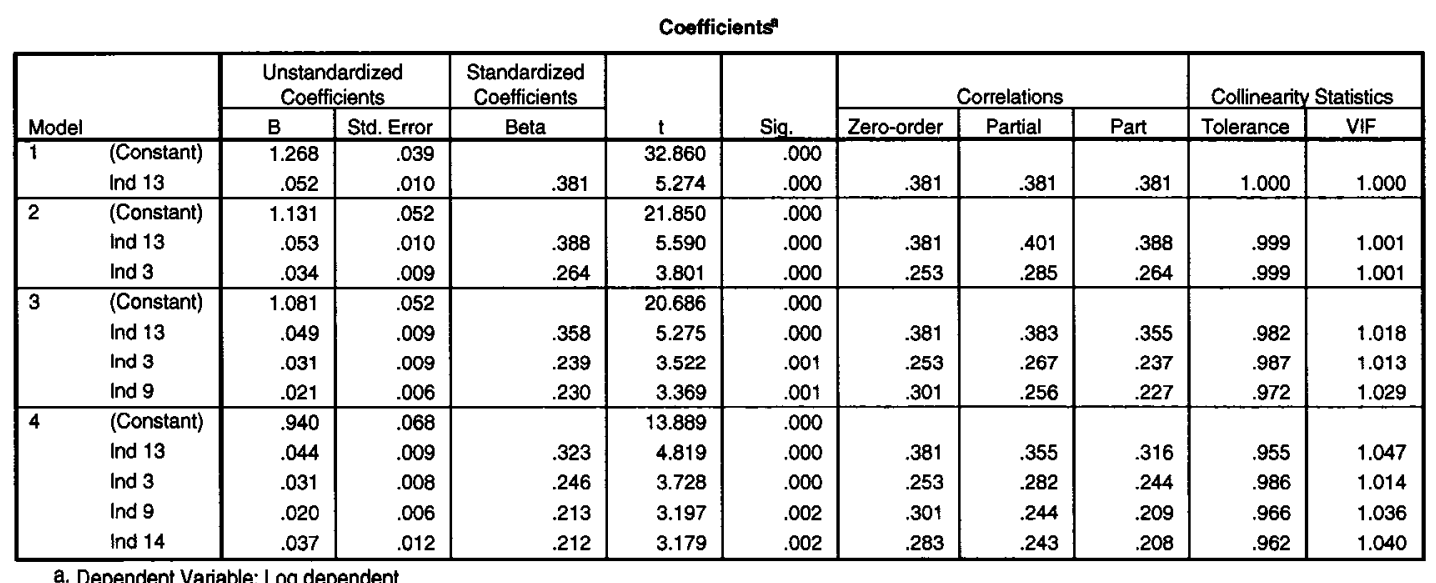

Table 6.11: Research Question 1: Regression Model Summary

\begin{tabular}{l|r|r|r|r|}
\hline Model & R & R Square & $\begin{array}{c}\text { Adjusted R } \\
\text { Square }\end{array}$ & $\begin{array}{r}\text { Std. Error of } \\
\text { the Estimate }\end{array}$ \\
\hline 1 & $.330(\mathrm{a})$ & .109 & .104 & .12685 \\
2 & $.436(\mathrm{~b})$ & .190 & .180 & .12136 \\
3 & $.491(\mathrm{c})$ & .241 & .227 & .11783 \\
4 & $.530(\mathrm{~d})$ & .281 & .263 & .11505 \\
\hline
\end{tabular}
a Predictors: (Constant), Ind 13
b Predictors: (Constant), Ind 13, Ind 3
c Predictors: (Constant), Ind 13, Ind 3, Ind 9
d Predictors: (Constant), Ind 13, Ind 3, Ind 9, Ind 14
e Dependent Variable: LOG (base e) DEP

Table 6.10 and Table $6.11^{29}$ show the regression coefficients and the regression model summary that are obtained. The $\mathrm{R}$ values for each model estimate the coefficients of multiple correlation. It indicates the degree of linear relationship between the observed and predicted values of Success. As we can see, the values of $R$ for all the above four models produced by the stepwise regression procedure belong to the range $(0,1)$. In general, greater the $\mathrm{R}$ values, the stronger the relationships are. The proportion of variation in Success explained by the regression model is estimated by the $\mathrm{R}$ squared 
values. The $\mathrm{R}$ squared values also lie in the range $(0,1)$. Model 4 has the highest value of $\mathrm{R}$ squared amongst all the four models. It has four predictors: Ind13, Ind3, Ind9, and Ind14. Thus, stepwise regression, in this example, reveals only four variables as predictors of Success. This is consistent with the commonly used idea that there should not be too many predictors in a good multiple regression model. In order to better reflect the goodness of fit of the model, the adjusted $\mathrm{R}$ squared is used. The adjusted $\mathrm{R}$ squared for Model 4 is also the highest amongst all the four models.

In Table 6.10, the B values in the "unstandardized coefficients" column are the coefficients of the estimated regression model. Thus, in this study, the estimated model is:

$$
\ln (\text { Success })=.940+0.044(\text { Ind 13) }+\mathbf{0 . 0 3 1}(\text { Ind 3) }+.020(\text { Ind 9) }+.037(\text { Ind 14) }
$$

In the above equation:

Ind 3 represents Customer Commitment Ind 9 represents Control Ind 13 represents Societal Culture Ind 14 represents Training and Learning

The standardized coefficients (the Beta values) are an attempt to measure the strength of one regression predictor relative to another. They are measured in standard deviation units. In this study, in Model 4, the largest Beta is .323 and the smallest Beta is .212. This indicates that a 1 standard deviation increase in Ind13 (or Ind14) would lead to .323 units (or .212 units) increase in standard deviation of Success.

\footnotetext{
${ }^{29}$ Figures 6.10 and 6.11 and alike figures in this Thesis are obtained as outputs from SPSS.
} 
If, prior to performing the regression analysis, we had transformed all the outcome and the predictor variables to a standard set of scores called the $\mathrm{z}$ scores, we would get the Beta coefficients as the unstandardized coefficients.

By looking at the t-statistics in Table 6.10, we can estimate the importance of one variable relative to another in the model. The general rule of thumb used for looking at $t$ values is to look for $t$ values below -2 or above +2 . Here all the variables Ind13, Ind3, Ind9, and Ind14 have $t$ value well above +2 .

Also, the collinearity statistics indicates that in Model 4, all the VIF values are well below the threshold of 2 (VIF values greater than 2 are considered problematic), and all the tolerance values are above the threshold of 0 . This indicates that with this model, multicollinearity is not a problem.

Table 6.12 summarizes the results of analysis of variance corresponding to each of the 4 models. Corresponding to each model, the sum of squares, degrees of freedom, and mean square are shown. The amount of variation that can be accounted for by each of these four models is shown in Regression row of the table, the amount of variation that cannot be accounted for by each of these models is shown in the Residual row, and the total variation (i.e., the sum of the variations accounted for by both the Regression, and the Residual) is also shown thereafter for each of the four models. Amongst all the four models, Model 4 has the largest regression sum of squares compared to the residual sum of squares. It has the least residual compared to the other three models. The F statistic results in the table reveal that the independent variables Ind13, Ind3, Ind9, and Ind14 are 
good (significant) predictors of variation in Success, because the significance value (i.e., .000 ) is less than .05 (the test of significance cut off value).

The residual statistics, i.e., the minimum, maximum, mean, and standard deviation, for the predicted, and the residual (i.e., the difference in the value that is observed, and the one that is predicted), and the standardized values (those that have been normalized to have a mean of 0 and a standard deviation of 1) are shown in Table 6.13.

Table 6.12: Research Question 1: ANOVA Table from Regression Analysis

\begin{tabular}{|c|c|c|c|c|c|c|}
\hline \multicolumn{7}{|c|}{ ANOVA } \\
\hline Model & & $\begin{array}{l}\text { Sum of } \\
\text { Squares }\end{array}$ & df & Mean Square & $F$ & Sig. \\
\hline \multirow[t]{3}{*}{1} & Regression & .454 & $\overline{1}$ & .454 & 27.815 & $.000^{\mathrm{a}}$ \\
\hline & Residual & 2.679 & 164 & .016 & & \\
\hline & Total & 3.133 & 165 & & & \\
\hline \multirow[t]{3}{*}{2} & Regression & .672 & 2 & .336 & 22.270 & $.000^{\mathrm{b}}$ \\
\hline & Residual & 2.461 & 163 & .015 & & \\
\hline & Total & 3.133 & 165 & & & \\
\hline \multirow[t]{3}{*}{3} & Regression & .833 & 3 & .278 & 19.573 & $.000^{c}$ \\
\hline & Residual & 2.299 & 162 & .014 & & \\
\hline & Total & 3.133 & 165 & & & \\
\hline \multirow[t]{3}{*}{4} & Regression & .969 & 4 & .242 & 18.031 & $.000^{d}$ \\
\hline & Residual & 2.164 & 161 & .013 & & \\
\hline & Total & 3.133 & 165 & & & \\
\hline
\end{tabular}
a. Predictors: (Constant), Ind 13
b. Predictors: (Constant), Ind 13, Ind 3
c. Predictors: (Constant), Ind 13, Ind 3 , Ind 9
d. Predictors: (Constant), Ind 13, Ind 3, Ind 9, Ind 14
e. Dependent Variable: Log dependent 
Table 6.13: Research Question 1: Residuals Statistics from Regression Analysis

\begin{tabular}{|l|r|r|r|r|r|}
\hline & \multicolumn{1}{|c|}{ Minimum } & Maximum & \multicolumn{1}{|c|}{ Mean } & Std. Deviation & \multicolumn{1}{c|}{ N } \\
\hline Predicted Value & 1.2031 & 1.6049 & 1.4648 & .07651 & 166 \\
Std. Predicted Value & -3.412 & 1.829 & .001 & .998 & 166 \\
Standard Error of & .010 & .042 & .019 & .006 & 166 \\
Predicted Value & & & & \\
Adjusted Predicted Value & 1.1874 & 1.6058 & 1.4651 & .07599 & 166 \\
Residual & -1.45311 & .23446 & -.00831 & .15946 & 166 \\
Std. Residual & -12.535 & 2.023 & -.072 & 1.376 & 166 \\
Stud. Residual & -12.369 & 2.089 & -.072 & 1.381 & 166 \\
Deleted Residual & -1.45311 & .25005 & -.00860 & .16279 & 166 \\
Stud. Deleted Residual & -12.369 & 2.111 & -.074 & 1.386 & 166 \\
Mahal. Distance & .284 & 21.076 & 3.958 & 3.747 & 166 \\
Cook's Distance & .000 & .426 & .011 & .039 & 166 \\
Centered Leverage Value & .002 & .128 & .024 & .023 & 166 \\
\hline
\end{tabular}

a Dependent Variable: Log (base e) dependent

Histogram

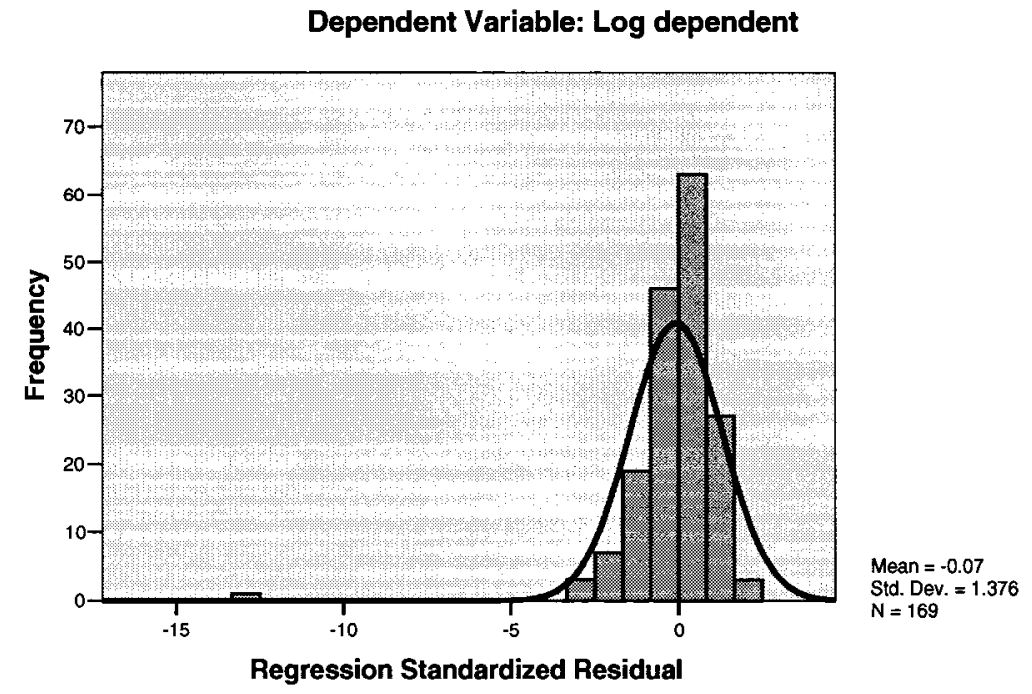

Figure 6.1: Histogram Showing Frequency Versus Regression Standardized Residual 
Ideally, for a good regression model, the residuals should follow a normal distribution. Figure 6.1 shows the histogram of the regression standardized residual, which is the value of the difference between the observed value and that predicted by the model for the dependent variable, Success. As the shape of the histogram in Figure 6.1 closely follows the shape of the normal curve, it is acceptable. Similarly, from Figure 6.2 we see that the P-P plotted residuals follow the 45-degree line. Thus we see that neither the histogram nor the P-P plot indicates that the normality assumption in linear multiple regression is violated. Figure 6.3 shows that the error variance is more or less constant.

Normal P.P Plot of Regression Standardized Residual

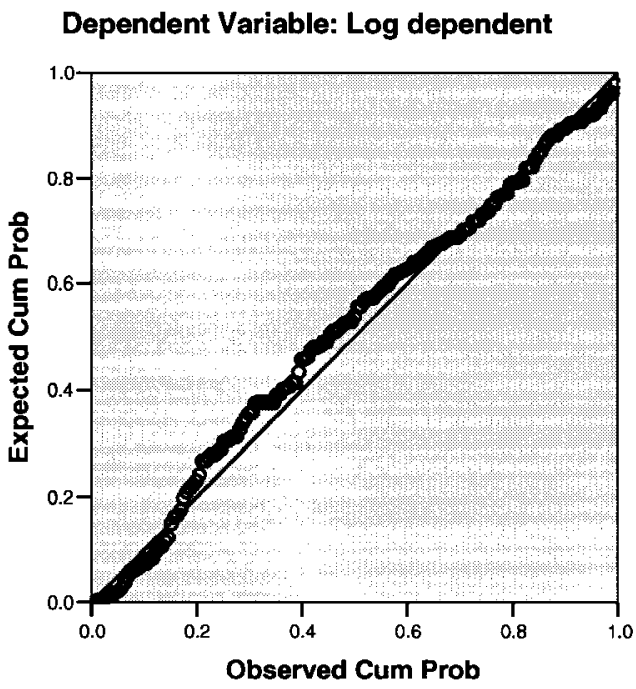

Figure 6.2: Normal P-P Plot of Regression Standardized Residual 


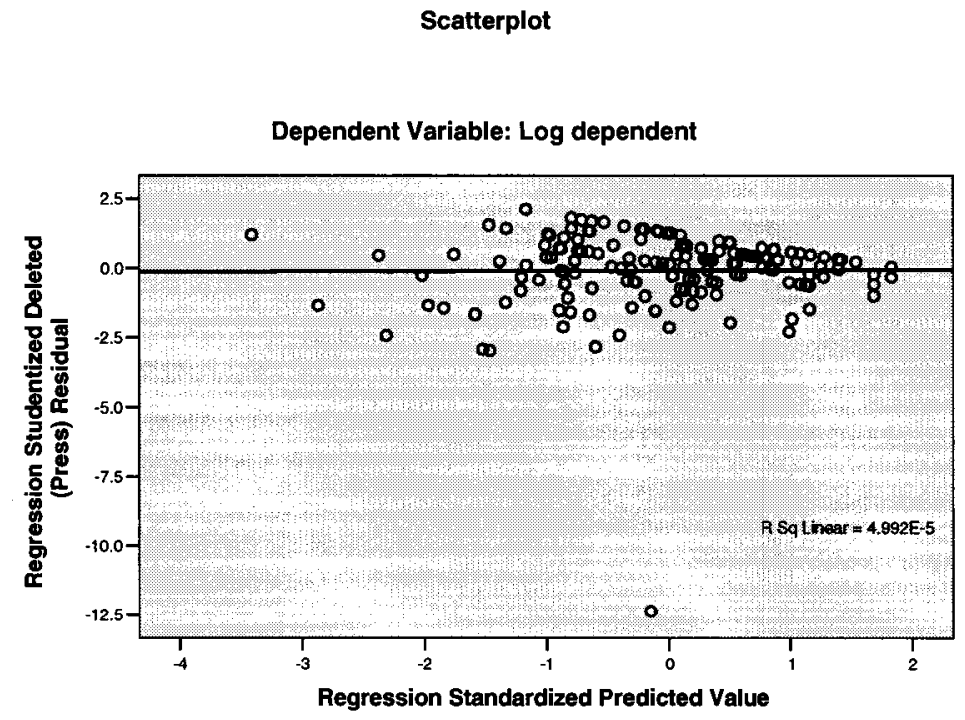

Figure 6.3: Check for error variance

In exactly similar manner, stepwise linear multiple regression was also run on the data taking each of the five dependent variables, D1, D2, D3, D4, and D5 individually as representatives of Success, instead of consolidating them all together to represent Success, as was done thus far. The regression models that are obtained for each of D1, D2, D3, D4, and D5 are presented in Table 6.14. The detailed results of regression are omitted from here in the interest of maintaining the readability of this Chapter. The detailed results are given in Section E.2 in Appendix E. They are obtained by exactly following the steps mentioned in the previous discussions. 
Table 6.14: Regression Models for D1, D2, D3, D4, and D5

\begin{tabular}{|l|l|l|}
\hline & \multicolumn{1}{|c|}{ Predictors } & \multicolumn{1}{c|}{ Regression Model } \\
\hline 1 & Ind13, Ind14 & D1 $=2.343+.255($ Ind13) $+.197($ Ind14) \\
\hline 2 & Ind14, Ind9, Ind2 & $\ln (\mathrm{D} 2)=.626+.098($ Ind14 $)+.048($ Ind9) $+.052($ Ind2) \\
\hline 3 & Ind3, Ind7 & $\ln (\mathrm{D} 3)=1.232+.036($ Ind3 $)+.038($ Ind7) \\
\hline 4 & Ind3 & D4 $=4.24+.013($ Ind3) \\
\hline 5 & Ind4, Ind7, Ind3 & $\ln (\mathrm{D} 5)=.722+.079(\operatorname{Ind} 4)+.052(\operatorname{Ind} 7)+.033($ Ind3) \\
\hline
\end{tabular}

\subsubsection{Success Factors Suggested by Respondents}

The respondents were also requested to list up to five additional success factors. As it was an optional question, some of the participants responded to it, while some did not. A visual inspection of all the listed factors reveal the following high level categories to which all the factors can be classified. The comments written against each of these high level dimensions below are based on the synthesis of the responses from the survey respondents. The raw forms of the responses, as written by the respondents, are listed in Appendix D. However, I have synthesized them below by providing my own perspective whenever required and deemed appropriate. It should be noted that many of the success factor dimensions/categories that we have identified below overlap with those of the ones that we had previously identified, but the individual factors within them are different. Therefore, we list all the dimensions even if they were previously identified so that we do not lose any data that we have obtained. However, it should be remembered that what is important for us is the identification of the major dimensions of the success factors. The list of factors contributing to any of the dimensions that we have identified could be large 
(not restricted to the ones we had in the questionnaire and the ones brought forward through the open-ended questions).

\section{Customer centric issues}

Customer centric issues constitute important success factors. These were not only revealed from our search of existing literature, but also from the open-ended responses from the survey participants. Our open-ended survey responses reveal that they are perhaps the most emphasized of all factors. In the words of one of the survey respondents:

"Customer must participate effectively in process and be able to balance business and technical goals. If not, you are doomed to fail over and over... or at minimum do Agile very badly".

There should not only be continuous customer involvement and collaboration, but there should also be an understanding of the process by the customer. There should be strong buy-in from the customers. The customers (or even business area or product owners) should not only be involved and committed but should also be competent and authorized to make decisions. The involvement of the customers should be of different forms including frequent visits and teleconferences. One of the respondents suggests having non-colocated customers without any further elaboration.

In fact, the commitment should come not only from the customers but from the business areas in the general sense. The commitment should be significant. The business areas or the customers should participate in the prioritization of all development activities. The customers should have no incentives that are contradictory to use of agile approaches. 
Some of the respondents suggested that customers should have trust on the developers of the product. This trust should be from all respects particularly when it comes to adding additional features for the betterment of the product being developed, without their consent or without any restriction such as the initial contract or statement of work.

One of the respondents has also mentioned that the maturity of the clients is also very important. In his/her words:

"Some just do not want to cooperate - they try to buy software like groceries".

One of the respondents also suggested without any further elaboration that having a track record with the client is also a success factor.

\section{Learning from failure}

An important dimension suggested by a few respondents is that learning from failure is an important success factor. It is important to note, analyze, and understand what failed earlier and why, perform a root cause analysis of the core problems if something proves to be problematic. All the problems should be analyzed and core problems should be identified and analyzed in detail.

\section{Training and learning}

Mentoring, training, and learning are considered important success factors by many respondents that were surveyed. One of the respondents even suggested having full time Agile coach(es). One respondent suggested having external coaching services to be important. 
Several respondents suggested programming in pairs. Pair programming has many benefits which include discussing designs, understanding them better, and efficiently sharing knowledge between different team members.

\section{Competency}

Competency in different forms has been identified as success factors by many respondents. The individual capabilities of the team members, having skilled developers, and craftmanship were suggested as being important success factors. The competency should not only be from the technical side but should also be from the business side. The competency factor is not only for the developers but should also be for the customers. The customers should be technically sound.

The team members should be talented, smart, experienced with software development practices in general and agile approaches in particular, familiar with the technical platform, have sound domain and business knowledge, and should be capable of being their own customers when required.

\section{Personal characteristics}

In addition to competency, personal characteristics such as having intellect, ability and initiativeness to take up responsibility, having good principles, being passionate, not being a perfectionist, being able to self-criticize, having respect for other individuals, humility, having courage for bringing up the truth about the project fate, and willingness to work together and program in pairs (said to be difficult among the experienced team members) were suggested as important success factors. 


\section{Communication}

Communication as a success factor was suggested by many respondents in different forms. It was suggested that there should be open and continuous feedback mechanism between developers themselves, and between developers and customers. It was suggested to set up communication rules, use information radiators such as wall charts and postcards. Usage of simple communication tools such as Wiki was also suggested by a respondent.

\section{Timing issues}

Timing issues such as time to delivery and time to market were suggested to be important success factors. One of the respondents suggested that "Time Boxing is probably the most important thing". It is important to segment the time into short frames, scale down the requirements to be very specific so that they can be delivered in short time frames. One of the respondents mentioned that:

"We estimate task duration and have the customer choose tasks \& priorities".

\section{Corporate culture and management issues}

Different aspects related to corporate culture and management issues were suggested as success factors. Of all one of the most emphasized factors is that there should be management support from all levels in favor of agile practices. The management buy-in and support especially from the highest level should be present. The management should understand the principles and values behind the agile practices and should be supportive of them and be committed. It was suggested that the level of maturity of the management in the organizations in the field of agile development is important. 
The organization culture should be supportive of establishing an agile value system instead of following a pre-defined set of practices advocated by the agile philosophy. This requires that all members in the organization including the developers, management, QA people and data owners should be agile. This in turn requires that ideally there should be buy-in from most stakeholders.

The organization culture should be free from all politics, support adaptability to rapid changes involved with agility, and all development should be driven by the business necessities and not be driven by IT/IS/IM. The organization culture should have a firmly established customer-vendor relationship.

\section{Use of tools}

Using good and appropriate tools is also suggested by many respondents to be a success factor. Using agile specific project management tools such as Rally, using ProjectCards for project organization, using tools that help in continuous integration and governance were suggested. One respondent also suggest that properly using the tools is also an important success factor.

\section{Team characteristics}

Characteristics of the team such as having a dynamic team that works in groups, works towards a common goal, and is empowered to take decisions on their own are suggested to be important success factors. Having matured members in the team is suggested as a success factor. The team members should believe in the motivation behind the agile practices, should be matured, capable of programming in pairs (pair programming), should be adaptive (particularly for the test team because they are subject to continuous 
changes in test plans), and the development members should be familiar with the project model. The importance of pair programming can be estimated by the fact that a number of suggestions were received for it. One of the respondents has suggested that:

"Paired programming is a critical success factor in keeping the teams up to speed on development issues, and cross training".

The stability of the team was considered to be important. Openness and honesty of the team members about the project status was also considered to be an important success factor. In the words of one of the respondents:

"Open and honest about the project status ...good or bad to recognize problems earlier".

\section{Planning}

The suggested success factors centering on planning include performing efficient estimations, and maintaining their integrity. One of the respondents also suggested maintaining "lightweight written plans (like burndown charts)". Interestingly, the suggestions from these respondents are not completely against maintaining any plans but for maintaining some plans that are not heavily weighed with strong processes.

\section{Control}

Some of the suggested success factors are centered on project control. Most of the suggested factors support a qualitative control mechanism. They are listed below:

- Having weekly showcases to show progress to customer

- Disciplined yet light documentation

- Daily Standup 
One of the respondents suggested that the progress should be visible to everyone including the customer. However, this respondent suggests "completed story points per iteration" (a quantitative measure) to measure progress.

\section{Other factors}

Some of the other suggested success factors that are not classified to belong to any of the above mentioned categories include:

- $\quad$ Having full-time team members on the project.

- Staff changes through attrition or reorganizing

- Criticality of the software - what is at stake?

- $\quad$ Disciplined yet light processes

- Quantitative demonstration of benefits of agile (DSDM) - independent metrics case study

- Not working on systems with closed list of modules

- $\quad$ Full time iteration manager (internally-facing project manager)

- Business priorities change, conflicts between availability of business versus IT technical staff

- Dynamism of the project - how many changes in requirements per month

Close inspection of all the suggested factors (dimensions) mentioned in the above categories shows that most of the dimensions were already considered in the study as part of the closed-ended questions. Of course, even though most of these high level dimensions overlap with the ones that we considered already, they suggest different new 
aspects for measuring those dimensions. These serve as valuable resources for future researchers and practitioners because many of them cannot be found in existing pieces of literature. They are obtained here from the first hand experience of practitioners working on agile software development projects.

It should be noted that the list of success factors already considered as part of the closed-ended questions were prepared based on literature review and review of a plenty of experience reports. Therefore, it is not surprising that most of the important dimensions of success factors were already captured. The different questions that we had already considered to be measuring the high level dimension were revealed from existing literature.

The new dimensions, in addition to those that were already considered, that are revealed from the open-ended questions responses are learning from failure, timing issues, use of tools, and other team characteristics. It should be noted that some aspects of team characteristics such as the team size and team distribution were already considered in the hypothesized theoretical framework. Other team characteristics are also revealed through the responses to the open-ended questions which were not revealed by our success factors literature survey. Similarly, the decision time aspect of timing issues were already considered in the theoretical framework.

\section{Technical factors}

In addition to the above, many of the factors that were suggested could be classified as technical factors. Although, in this study, we focused only on organizational and people 
factors, we thought of listing them below without any further elaboration, and not losing the valuable technical factor suggestions that are obtained from the survey:

- Integrating code constantly.

- $\quad$ Test Driven Development

- Type of Application Deliverable (In-House IT project vs Shrink Wrap Software)

- Goal-driven functionality and User Interface design

- Technical support environment

- $\quad$ Automated Testing: allows for shared code ownership (developers can feel free to make changes, having unit/functional/integration tests as safeguards)

- Getting the team test infected using xUnit and some kind of mocking tool

- Writing unit tests before writing code

- $\quad$ Refactoring the code often

- Mocks are important, if you can't mock, you can't unit test

- User Interface should develop along with the software

- Design architecture stable

- (Known) architecture

\subsubsection{Discussions}

We have seen that solely from the analysis of the quantitative data obtained, nine factors emerge to have statistically significant relationship with Success, whereas five do not. 
The ones that emerge to be so are customer satisfaction, customer collaboration, customer commitment, decision time, corporate culture, personal characteristics, societal culture, and training and learning. Among the ones that did not show significant relationship are team distribution, team size, planning, technical competency, and communication and negotiation. As we will see in Chapter 7, based on the citations from the existing pieces of literature in this area, and the results obtained from the analysis of the qualitative responses obtained through the open-ended questions, all the nine factors that show statistically significant relationship with Success are strongly justifiable. Further statements of support from the literature, in addition to the ones already given in Chapter 4, are given in an attempt to further establish the results. However, after analyzing the results obtained corresponding to the other five factors that did not emerge as significant success factors, using logical justification, using support from the previously published literature, and using the responses obtained through the open-ended questions, we feel that there are in-depth studies that should be performed to establish or reject the observed results through this study.

Finally, as we have seen, multiple regression modeling reveals that out of all the factors, only four emerge to be the best predictors of Success. These four predictors are: customer commitment, control, societal culture, and training and learning. Since we were not able to find any literature support comparing the different potential success factors we considered, let us intuitively examine the sensibility of this observation, although attempting such an intuitive reasoning is difficult. Logically, if customers remain committed in a project, they will closely collaborate, and are likely to be satisfied. Having customer adequately committed to a project (particularly if they are collocated 
with the rest of the development team) will also make sure that there will be lots of internalized, informal planning between the development teams and the customers, thereby relieving the customers or the development team to depend on formal documented plans. In other words, having customers committed to a project is very important. The other predictor revealed from regression modeling is the societal culture. From one perspective, corporate culture is strongly influenced by the societal culture. For example, it is unlikely that in an oppressive societal culture, the culture of an organization operating in that society will be non-bureaucratic, the management will be supportive of the decisions of the developers, and so on. Of course, having the right corporate culture does not necessarily mean that there are "right type" of people in the society. But societal culture does have a strong influence on the corporate culture. Also, the personal characteristics of people, their communication and negotiation mechanisms, and their other non-technical competencies are strongly influenced by the societal culture. Training and Learning are also perhaps very important. If there is a suitable training and learning mechanism, the technical competencies of the employees will strongly be governed by it. This is particularly true for those who are in the low experience groups. Control is another predictor that we have obtained. If there are efficient qualitative control mechanisms in a project, the decision time would also be reduced, and there is likely to be informal planning processes. It should also be observed that there is no significant logical overlap between the four predictors of Success that we have obtained, indicating that these four predictors are an absolute minimum set of predictors that would help in predicting success in a project. We also expected team factors such as team size and team distribution to show up in the list of predictors. However, the reason they are not in the 
list of predictors that we have obtained could be attributed to the fact that there are varying opinions regarding these issues as to whether or not they are important as determinants of success. We will examine such issues in considerable detail in Chapter 7.

Further detailed discussions justifying the results obtained from the data analysis for this research question can be found in Chapter 7. In the interest of not being repetitive in our discussions regarding these, we do not address them any further in this Chapter.

\subsubsection{Research Question 2}

We recall that in Research Question 2 our goal is to determine the critical changes that are required for adopting agile software development practices in projects practicing traditional life-cycle-based software development and rank the changes according to their level of importance. The data analysis for these studies was performed using two datasheets showing separate lists of changes required corresponding to which every survey respondent had ranked his/her judgment of the level of importance of each of the change items on a 5-point Likert scale. The results of the statistical analyses are summarized below.

It should be noted that, similar to as mentioned in Section 6.2.1, many of the change dimensions/categories that we have identified below overlap with those of the ones that we had previously identified, but the individual change items within them are different. Therefore, we list all the change dimensions even if they were previously identified so that we do not lose any data that we have obtained. However, it should be remembered that what is important for us is the identification of the major dimensions of change. The list of individual change items contributing to any of the dimensions that we have 
identified could be large and not restricted to the ones we had in the questionnaire and the ones brought forward through the open-ended questions.

\subsubsection{Descriptive Statistics}

As we did for Research Question 1, for Research Question 2 as well we first performed descriptive statistics to gain an understanding of the overall distribution of the survey responses. We determined the various statistics such as mean and standard deviation corresponding to each change. The means obtained from this step are then used to come up with the desired rankings later on.

Table 6.15 shows the means and standard deviations corresponding to the data obtained from the respondents for each of the different change item variables. It shows that, on an average, the respondents felt that the majority of the change items are in the range ("Somewhat Important", "Very Important"), whereas a few are in the range ("Neutral", "Somewhat Important"), although in the later case the values are close to "Somewhat Important".

In addition to the means and standard deviations corresponding to each of the change item variables presented in Table 6.15, Section E.3.1 in Appendix E, we also computed the $95 \%$ confidence interval, median, variance, minimum value, maximum value, skewness and kurtosis. The data in each case follows close to normal distribution. Although there is slight skewness or kurtosis detected for some of the variables, they are not significantly large. 
Table 6.15: Research Question 2: Summarized Descriptive Statistical Results

\begin{tabular}{|c|c|c|c|}
\hline Variables & $\mathbf{N}$ & Mean & $\begin{array}{c}\text { Std. } \\
\text { Deviation }\end{array}$ \\
\hline $\mathrm{C} 1$ & 165 & 4.19 & 0.90 \\
\hline $\mathrm{C} 1.1$ & 165 & 4.04 & 1.32 \\
\hline $\mathrm{C} 1.2$ & 165 & 4.25 & 1.08 \\
\hline $\mathrm{C} 1.3$ & 165 & 4.56 & 0.93 \\
\hline $\mathrm{C} 1.4$ & 165 & 3.74 & 1.38 \\
\hline $\mathrm{C} 1.5$ & 165 & 4.38 & 1.13 \\
\hline $\mathrm{C} 2$ & 165 & 4.35 & 1.01 \\
\hline $\mathrm{C} 2.1$ & 165 & 4.46 & 1.11 \\
\hline $\mathrm{C} 2.2$ & 164 & 4.23 & 1.11 \\
\hline $\mathrm{C} 3$ & 165 & 3.86 & 1.18 \\
\hline $\mathrm{C} 4$ & 165 & 4.19 & 0.93 \\
\hline $\mathrm{C} 4.1$ & 165 & 4.61 & 1.02 \\
\hline $\mathrm{C} 4.2$ & 164 & 3.64 & 1.36 \\
\hline $\mathrm{C} 4.3$ & 165 & 4.12 & 1.31 \\
\hline $\mathrm{C} 4.4$ & 165 & 4.39 & 1.05 \\
\hline
\end{tabular}

The consolidated distribution of the responses (in percentage figures) obtained from all the respondents for the different change items is captured in Section F.2 of Appendix F. Amongst all the change items, C4.1 (i.e., from heavily process centric to short, iterative, test-driven, and people centric development) was considered by the largest percentage (amongst all the percentage figures displayed in the table) of respondents (roughly $77 \%$ ) to be very important.

\subsubsection{Test of Internal Consistency Reliability}

As part of Research Question 2 as well, we try to understand the internal consistency of the change items. Table 6.16 shows the reliability statistics using the four consolidated change items $\mathrm{C} 1, \mathrm{C} 2, \mathrm{C} 3$, and $\mathrm{C} 4$, and Table 6.17 shows the inter-item correlations corresponding to all possible pairs formed by $\mathrm{C} 1, \mathrm{C} 2, \mathrm{C} 3$, and $\mathrm{C} 4$. It can be seen that the 
value of Cronbach's alpha is .822 , which implies that there is $82.2 \%$ of internal consistency, whereas $17.8 \%$ inconsistency in test scores. Also, since the value of Cronbach's alpha is well above the acceptable cut of limit of .6 and the adequate cut of limit of .7, there is significant internal consistency between the test items.

The reliability statistics and the correlations between items constituting to form the higher level change variables $\mathrm{C} 1, \mathrm{C} 2$, and $\mathrm{C}^{30}$ are presented in Section E.3.3 of Appendix E. The value of Cronbach's alpha for items within C1 is .819 , that corresponding to the items within $\mathrm{C} 2$ is .799 and that corresponding to the items within C4 is .786. All of these values of Cronbach's alpha are well above the adequacy cut off limit of .7.

Table 6.16: Research Question 2: Reliability Statistics

\begin{tabular}{|c|c|c|}
\hline & $\begin{array}{c}\text { Cronbach's } \\
\text { Alpha Based }\end{array}$ & \\
$\begin{array}{c}\text { Ononbach's } \\
\text { Alpha }\end{array}$ & $\begin{array}{c}\text { Standardized } \\
\text { Items }\end{array}$ & N of Items \\
\hline .822 & .833 & 4 \\
\hline
\end{tabular}

Table 6.17: Research Question 2: Inter-Item Correlation Matrix

\begin{tabular}{|l|r|r|r|r|}
\hline & \multicolumn{1}{c|}{$\mathrm{C} 1$} & \multicolumn{1}{c|}{ C2 } & \multicolumn{1}{c|}{ C3 } & \multicolumn{1}{c|}{ C4 } \\
\hline $\mathrm{C} 1$ & 1.000 & .661 & .487 & .692 \\
$\mathrm{C} 2$ & .661 & 1.000 & .457 & .521 \\
$\mathrm{C} 3$ & .487 & .457 & 1.000 & .509 \\
$\mathrm{C} 4$ & .692 & .521 & .509 & 1.000 \\
\hline
\end{tabular}

${ }^{30}$ It should be noted that $\mathrm{C} 3$ is measured with only a single item. 


\subsubsection{3 t-test}

We performed one sample t-test to determine if the means of each of the variables $\mathrm{C} 1$, $\mathrm{C} 2, \mathrm{C} 3, \mathrm{C} 4$ and each of the variables constituting them significantly differed from the specified constant "3" (Neutral), which is the mid-point of the 5-point Likert scale. Table 6.18 shows the results of the one sample t-test for $\mathrm{C} 1, \mathrm{C} 2, \mathrm{C} 3$, and $\mathrm{C} 4$. As we can see, the significance value in each of these cases is .000 , which is less than .05 (the significance level we have used for all the studies in this Thesis.) This indicates that the observed means are significantly different from Neutral. In fact, the mean difference corresponding to each of these change variables shows that the difference is significantly greater than the test value. The significant difference between the observed mean and the test value is also substantiated by the fact that the $95 \%$ confidence interval (range) corresponding to each of these change items does not contain the value, 0 .

In addition to Table 6.18 , the one sample t-test results for each of the variables constituting the higher level variables $\mathrm{C} 1, \mathrm{C} 2$, and $\mathrm{C} 4$ are also presented in Section E.3.2 in Appendix E. The one sample t-test results for all cases shows that the observed means for each of those variables constituting the higher level variable $\mathrm{C} 1, \mathrm{C} 2$, and $\mathrm{C} 4$ are significantly greater than the test value, 3 . 
Table 6.18: Research Question 2: One-Sample t-Test

\begin{tabular}{|c|c|c|c|c|c|c|}
\hline & \multicolumn{6}{|c|}{ Test Value $=3$} \\
\hline & \multirow[b]{2}{*}{$t$} & \multirow[b]{2}{*}{ df } & \multirow[b]{2}{*}{ Sig. (2-tailed) } & \multirow[b]{2}{*}{$\begin{array}{c}\text { Mean } \\
\text { Difference }\end{array}$} & \multicolumn{2}{|c|}{$\begin{array}{c}95 \% \text { Confidence Interval } \\
\text { of the Difference }\end{array}$} \\
\hline & & & & & Lower & Upper \\
\hline$\overline{C 1}$ & 17.073 & 164 & .000 & 1.19273 & 1.0548 & 1.3307 \\
\hline $\mathrm{C} 2$ & 17.060 & 164 & .000 & 1.34545 & 1.1897 & 1.5012 \\
\hline C3 & 9.380 & 164 & .000 & .86061 & .6794 & 1.0418 \\
\hline $\mathrm{C} 4$ & 16.447 & 164 & .000 & 1.19192 & 1.0488 & 1.3350 \\
\hline
\end{tabular}

\subsubsection{Ranking}

For determining the importance of the change items and ranking them, we use the means of all the responses from all the respondents for each change item presented in Table 6.15 earlier. Based on all the mean scores from all the change items, we rank them in the order of their importance. We note that all the variables pass the one sided t-test, as explained in the previous section. Table 6.19 presents the ranking of only the consolidated change items $\mathrm{C} 1, \mathrm{C} 2, \mathrm{C} 3$, and $\mathrm{C} 4 . \mathrm{C} 1$ and $\mathrm{C} 4$ have similar mean values but $\mathrm{C} 1$ has slightly less standard deviation value compared to $\mathrm{C} 4$. Therefore $\mathrm{C} 1$ was ranked slightly higher than C4. All the individual change items that constitute the composite change variables $\mathrm{C} 1$, C2, C3, and C4 are also ranked separately. Their results are presented in Table 6.20.

Although one sample t-test provides us an estimate of whether the change item means are significantly greater than the fixed test value 3 (corresponding to Neutral of the scale), it does not provide us any information about whether the means of two successively occurring change items in the ranked list of items shown in Tables 6.19 and 6.20 are significantly different from each other. To test this, we performed pair-wise ttest, the results of which are provided in Appendix E, Tables E.30 and E.31. In Table 
E.30, the pairs considered were $\mathrm{C} 1-\mathrm{C} 2, \mathrm{C} 1-\mathrm{C} 4$, and $\mathrm{C} 3-\mathrm{C} 4$. From this table, we observe that the mean of $\mathrm{C} 2$ is significantly different from that of $\mathrm{C} 1$, the mean of $\mathrm{C} 3$ is significantly different from $\mathrm{C} 4$, whereas the mean of $\mathrm{C} 4$ is not significantly different from C1. Similarly, from Table E.31, except the pair C1.2-C1.5, the means of all the other successively occurring pairs in the ranked list were found to have a significant difference. Therefore, we can conclude that although purely on the basis of the means of the change variables, we have ranked $\mathrm{C} 1.5$ higher than $\mathrm{C} 1.2$, there is not statistically significant difference in their means.

Table 6.19: Research Question 2: Ranking of the Consolidated Change Item Categories

\begin{tabular}{|c|c|c|c|c|}
\hline Rank & Variables & $\mathbf{N}$ & Mean & $\begin{array}{c}\text { Std. } \\
\text { Deviation }\end{array}$ \\
\hline 1 & $\mathrm{C} 2$ & 165 & 4.35 & 1.01 \\
\hline 2 & $\mathrm{C} 1$ & 165 & 4.19 & 0.90 \\
\hline 3 & $\mathrm{C} 4$ & 165 & 4.19 & 0.93 \\
\hline 4 & $\mathrm{C} 3$ & 165 & 3.86 & 1.18 \\
\hline
\end{tabular}

Based on the results from this study, we observe that the respondents opined that the Changes in Management Style are the most important (critical) changes required followed by Changes in Organization Culture, Changes in Development Processes, and Changes in Knowledge Management Strategies. Changes in Organization Culture and Changes in Development Processes have similar level of importance according to the survey results. 
Table 6.20: Research Question 2: Ranking of all the Individual Change Items in Each Change Category

\begin{tabular}{|c|c|c|c|c|}
\hline Rank & Variables & $\mathbf{N}$ & Mean & $\begin{array}{c}\text { Std. } \\
\text { Deviation }\end{array}$ \\
\hline \multicolumn{5}{|c|}{ C1 } \\
\hline 1 & C1.3 & 165 & 4.56 & 0.93 \\
\hline 2 & C1.5 & 165 & 4.38 & 1.13 \\
\hline 3 & C1.2 & 165 & 4.25 & 1.08 \\
\hline 4 & C1.1 & 165 & 4.04 & 1.32 \\
\hline 5 & C1.4 & 165 & 3.74 & 1.38 \\
\hline \multicolumn{5}{|c|}{ C2 } \\
\hline 1 & C2.1 & 165 & 4.46 & 1.11 \\
\hline 2 & C2.2 & 164 & 4.23 & 1.11 \\
\hline \multicolumn{5}{|c|}{ C3 } \\
\hline 1 & C3 & 165 & 3.86 & 1.18 \\
\hline \multicolumn{5}{|c|}{ C4 } \\
\hline 1 & C4.1 & 165 & 4.61 & 1.02 \\
\hline 2 & C4.4 & 165 & 4.39 & 1.05 \\
\hline 3 & C4.3 & 165 & 4.12 & 1.31 \\
\hline 4 & C4.2 & 164 & 3.64 & 1.36 \\
\hline
\end{tabular}

Within each of the above change item categories, the following is the ranking of the individual change items:

\section{Changes in Management Style}

- Rank 1: From command-and-control management to leadership-and-collaboration.

- Rank 2: From authoritative to collaborative and pluralistic decision making.

\section{Changes in Organization Culture}

- Rank 1: From solitary development attitudes of team members to that of working in teams.

- Rank 2: From non-customer-centric to customer-centric development. 
- Rank 3: From individually assigned roles to that of team-work. (Note: Based on the discussions above, although this change item have been ranked below the immediately preceding one (with Rank 2 above) purely on the basis of their respective means, there is no statistically significant difference of their means, as indicated by pairwise t-test).

- Rank 4: From policy and procedure based development culture to freedom of development and management by team members.

- Rank 5: From non-customer-centric to customer-centric development.

\section{Changes in Development Processes}

- Rank 1: From heavily process-centric to short, iterative, test-driven, and peoplecentric development.

- Rank 2: From lifecycle-based development to feature-driven evolutionary and iterative development.

- Rank 3: From contract-compliant to change-tolerant development.

- Rank 4: From standards compliance and measurement driven development to development under uncertainty.

\subsubsection{Changes Suggested by Respondents}

The respondents were also requested to list up to five additional changes they believe are required for adopting agile software development practices in organizations practicing traditional, plan-driven methodologies. Their detailed responses, as written by the 
respondents, are listed in Appendix D. However, as in the success factors survey, the changes below are synthesized from my own perspective based on the raw responses from the survey respondents. Being an optional question, some of the participants responded to the open-ended questions, while some did not. Close inspection of all the suggested changes reveals the following interesting dimensions of change.

\section{Changes in organization culture}

It was suggested that for agile software development there should be changes in organization culture that is supportive of continuous communication, knowledge sharing, increasing the culture of trust that is generally absent in many organizations, making the business sponsors responsible for prioritization of tasks, and planning of the releases, increasing the level of honesty and openness in communication in the organizations, having a suitable development team environment, involvement of all members in team activities, and increasing the culture of being adaptive rather than being predictive. It was also suggested that there should be "a right to errors culture", meaning that the organization culture should be such that errors can happen and the developers should not be penalized for the same.

\section{Changes in management style}

The changes in management style for agile approaches include having management permissive of making changes, having the openness in management activities, trusting their developers, shifting away from large-scale scope management to continuous (micro) scope management (without micromanaging the activities of the developers), risk and uncertainty acceptance by the management, having transparent radiators of project status 
and information, being honest while communicating about the progress of the project, and having non-dictating or non micromanaging management. According to the view of one of the respondents:

"Drive to Agile has to come from within the team - allow team to control not managers dictating method"

It was suggested that in agile practices the business analysts should be engaged from the early stages by the management. One of the respondents also cautioned that (since the agile approaches part away from quantitative measures of control) it can be disastrous for the project if there is a significantly diminished level of focus on progress measurement.

\section{Changes in knowledge management strategies}

Traditionally in heavy process-centric organizations there is a high emphasis on documentation as a medium for knowledge management. However, the agile practices tend to part away from heavy documentation centric development approach and emphasize more on "show working software" type development approach. As aptly put by forward by one of the respondents:

"One of the ability to reject structured documentation, such as a survey instrument, when it doesn't help my client".

\section{Changes in development processes}

The following changes in development processes favoring agile software development were suggested. The development processes in agile require a shared team room for pair programming and continuous interactions, performing continuous integration and mocks, 
performing test driven development (in which the test plans dictate further development activities), having tools that enable the kind of collaboration required in agile practices, reducing the scope of the projects, performing lots of prototyping, performing continuous builds in small steps and performing weekly releases, performing functional testing along with coding, and having the customers and other stakeholders to provide continuous feedback. It was also suggested that the development processes in agile software development methodologies require the architects to work alongside the programmers.

\section{Changes in personal characteristics}

Different suggestions for changing the personal characteristics of the team members were also received. It was suggested that individual team members should be self-motivated to make continuous changes as and when required, they should be knowledgeable about the business, they should be courageous, should develop the habit of collectively owning the solutions, they should earn the trust of the customers and other stakeholders, and they should have high degrees of tenacity. It was also suggested by a respondent that the technical folks in the teams should have sales skills. However, the reason for that is not apparent, but it is worth capturing.

\section{Changes in customer attitude}

It was suggested by a few respondents that adopting agile practices require changes in attitudes and behaviors of customers such as trusting the development team members and their willingness to negotiate when required (of course, the willingness to negotiate is also expected of the development team members). It was also suggested that the unlike in traditional plan-driven development, in agile development culture, the programmers 
should not be required to provide justification of each task they perform. It should be sufficient if they deliver the required working software.

\section{Changes in the knowledge and education}

All stakeholders (particularly the upper management) should be educated about the principles and values guiding the agile practices. The same holds for the development team members. They should in fact be knowledgeable about how to perform development using the agile methodologies. In the words of one of the survey respondents:

"If one person does not have the knowledge about agile development and is unwilling to learn, he can be a real stopper for the project. (management included)".

The above discussions show that most of the change categories were considered earlier. However, many new individual change items measuring the high level change categories are discussed. It should be noted that the list of changes already considered as part of the closed-ended questions have been prepared based on literature review and review of a plenty of experience reports. Therefore, it is not surprising that most of the important dimensions of change are already considered.

\subsubsection{Discussions}

We have seen that solely from the analysis of the quantitative data obtained, all the change dimensions that we considered show statistically significant results. The most important of all change dimensions is the changes in the management style. Changes in management style are followed by the changes in organization culture, changes in 
development processes, and changes in knowledge management strategies, in decreasing order of their importance. In the absence of the availability of any previous literature that ranks or prioritizes the different change items, we were not able to validate the results that we have obtained with the findings from the literature. However, in Chapter 7, we use qualitative logical reasoning and citations from the literature to support the reasonings/justifications we make in favor or against the observations we make. Further statements of support from the literature, in addition to the ones already given in Chapter 4, are given in an attempt to establish the results.

As we will see in Chapter 7, in the absence of any particular literature justifying our ranking results, based on the discussions from the existing pieces of literature in this area, and the results obtained from the analysis of the qualitative responses obtained through the open-ended questions, we find that all the four change categories are appropriately justifiable.

Further detailed discussions attempting to justify the results obtained from the data analysis for this research question can be found in Chapter 7. In the interest of not being repetitive in our discussions regarding these, we do not address them any further in this Chapter.

\subsubsection{Research Question 3}

Having ranked all the change items according to their levels of important, we proceed in Research Question 3 to rank all the challenges using the same strategies. Our intention is to determine the critical challenges that are involved in adopting agile software development practices in projects practicing traditional life-cycle-based software 
development. We want to arrive at a ranked list of challenges according to the level of importance (in the 1 to 5 point Likert scale) judged by the respondents that participated in the survey. The results of the statistical analyses are summarized below.

\subsubsection{Descriptive Statistics}

As we did for Research Questions 1 and 2, for Research Question 3 as well, performing descriptive statistics first was necessary to gain an understanding of the overall distribution of the data that we have. We determined the various statistics such as mean and standard deviation corresponding to each challenge item. In the same way as we did for change items, the mean values for each of those challenge items are then used to come up with the desired rankings later on, keeping in mind the results of significance of the t-tests.

Table 6.21 shows the mean values, the standard deviations, and the standard errors corresponding to each of the challenge variables.

Like before, in addition to these statistics of the challenge items, Section E.4 in Appendix E lists the $95 \%$ confidence interval values, the medians, variances, minimum values, maximum values, skewnesses and kurtosis of all the variables. We observe that corresponding to almost all of these variables the median value is close to the mean value, implying a close to normal distribution in each case. 
Table 6.21: Research Question 3: Summarized Descriptive Statistical Results

\begin{tabular}{|l|r|r|r|r|}
\hline & $N$ & Mean & Std. Deviation & $\begin{array}{c}\text { Std. Error } \\
\text { Mean }\end{array}$ \\
\hline Chal1 & 161 & 3.81 & 1.238 & .098 \\
Chal2 & 160 & 3.26 & 1.397 & .110 \\
Chal3 & 159 & 3.13 & 1.406 & .111 \\
Chal4 & 161 & 3.72 & 1.357 & .107 \\
Chal5 & 161 & 3.05 & 1.161 & .091 \\
Chal6 & 161 & 2.96 & 1.198 & .094 \\
Chal7 & 161 & 3.50 & 1.230 & .097 \\
Chal8 & 161 & 3.09 & 1.591 & .125 \\
Chal9 & 161 & 4.10 & 1.459 & .115 \\
Chal10 & 160 & 2.84 & 1.596 & .126 \\
Chal11 & 159 & 3.57 & 1.486 & .118 \\
Chal12 & 159 & 3.62 & 1.413 & .112 \\
Chal13 & 159 & 3.33 & 1.398 & .111 \\
Chal14 & 159 & 3.31 & 1.471 & .117 \\
Chal15 & 159 & 3.38 & 1.408 & .112 \\
Chal16 & 159 & 2.83 & 1.666 & .132 \\
Chal17 & 159 & 3.52 & 1.335 & .106 \\
Chal18 & 159 & 3.09 & 1.425 & .113 \\
Chal19 & 156 & 3.18 & 1.380 & .110 \\
\hline
\end{tabular}

The consolidated distribution of the responses (in percentage figures) obtained from all the respondents for the different challenge items is captured in Section F.3 of Appendix F. The results presented there are self-explanatory and are, thus, not explained further over here to maintain the brevity of this Chapter. Amongst all the challenge items, for example, Chal9 (i.e., upper management resistance) was considered by the largest percentage (amongst all the percentage figures displayed in the table) of respondents (roughly 55\%) to be very important. Another example is that of Chal5 (i.e., the differences in productivity between team members), which was voted by the majority of respondents to be somewhat unimportant challenge. 


\subsubsection{Test of Internal Consistency Reliability}

Table 6.22 and Table 6.23 shows the results of the test of internal consistency reliability involving all the 19 challenge items. The Cronbach's alpha value is .911 , implying that there is $91.1 \%$ internal consistency, and the remainder $8.9 \%$ of inconsistency. As the Cronbach's alpha value is significantly greater than the adequate cut-off value .7 , there is high degree of internal consistency between the test items.

Table 6.22: Research Question 3: Reliability Statistics

\begin{tabular}{|l|l|l|}
\hline $\begin{array}{l}\text { Cronbach's } \\
\text { Alpha }\end{array}$ & $\begin{array}{l}\text { Cronbach's } \\
\text { Alpha Based on } \\
\text { Standardized } \\
\text { Items }\end{array}$ & N of Items \\
\hline .911 & .911 & 19 \\
\hline
\end{tabular}

Table 6.23: Research Question 3: Summary Item Statistics

\begin{tabular}{|l|r|r|r|r|r|r|r|}
\hline & Mean & Minimum & Maximum & Range & $\begin{array}{c}\text { Maximum / } \\
\text { Minimum }\end{array}$ & Variance & N of Items \\
\hline Item Means & 3.336 & 2.833 & 4.090 & 1.256 & 1.443 & .113 & 19 \\
Inter-Item Correlations & .351 & .075 & .671 & .596 & 8.901 & .009 & 19 \\
\hline
\end{tabular}

For the Independent variable, Cronbach's alpha is 0.911 . This means that the test is 91\% Reliable and 9\% Unreliable. The inter-item correlations are shown in Section E.4.2 in Appendix E.

\subsubsection{3 t-test}

As we did for Research Question 2, we also performed one sample t-test to determine if the means of each of the variables Chal1, Chal2, Chal3, Chal4, Chal5, Chal6, Chal7, Chal8, Chal9, Chal10, Chal11, Chal12, Chal13, Chal14, Chal15, Chal16, Chal17, 
Chal18, and Chal19 significantly differs from the specified constant "3" (Neutral) corresponding to the mid-point of the 5-point Likert scale. Table 6.24 displays the results of the one sample t-test for each of the above mentioned variables.

As we can see, the significance value corresponding to each of the 11 variables Chal1, Chal2, Cha14, Chal7, Chal9, Chal11, Chal12, Chal13, Chal14, Chal15, and Chal17 is .000 , which is less than .05 (the significance level cut-off). Therefore, the observed means for these 11 variables are significantly different from Neutral.

It should also be observed that the $95 \%$ confidence interval corresponding to each of these challenges does not contain the value, 0 . On the other hand, the variables Chal3, Chal5, Chal6, Chal8, Chal10, Chal16, Chal18, and Chal19 have significance values greater than .05 , and the $19 \%$ confidence interval corresponding to each of them includes the value, 0 . So the difference between the sample mean and the test value is not significant.

\subsubsection{Ranking}

We consider the mean values listed in Table 6.21 for determining the importance of the challenge items and ranking them. Based on all the mean scores from all the challenge items, and considering only the variables that pass the one-sample t-test, we rank them in the order of their importance. The ranking of these variables according to the decreasing order of their importance is presented in Table 6.25. 
Table 6.24: Research Question 3: One-Sample t-Test

\begin{tabular}{|c|c|c|c|c|c|c|}
\hline & \multicolumn{6}{|c|}{ Test Value $=3$} \\
\hline & \multirow[b]{2}{*}{$\mathrm{t}$} & \multirow[b]{2}{*}{$\mathrm{df}$} & \multirow[b]{2}{*}{ Sig. (2-tailed) } & \multirow{2}{*}{$\begin{array}{c}\text { Mean } \\
\text { Difference }\end{array}$} & \multicolumn{2}{|c|}{$\begin{array}{c}95 \% \text { Confidence Interval } \\
\text { of the Difference }\end{array}$} \\
\hline & & & & & Lower & Upper \\
\hline Chal1 & 8.279 & 160 & .000 & .807 & .61 & 1.00 \\
\hline Chal2 & 2.320 & 159 & .022 & .256 & .04 & .47 \\
\hline Chal3 & 1.185 & 158 & .238 & .132 & -.09 & .35 \\
\hline Chal4 & 6.739 & 160 & .000 & .720 & .51 & .93 \\
\hline Chal5 & .543 & 160 & .588 & .050 & -.13 & .23 \\
\hline Chal6 & -.395 & 160 & .694 & -.037 & -.22 & .15 \\
\hline Chal7 & 5.188 & 160 & .000 & .503 & .31 & .69 \\
\hline Chal8 & .694 & 160 & .489 & .087 & -.16 & .33 \\
\hline Chal9 & 9.564 & 160 & .000 & 1.099 & .87 & 1.33 \\
\hline Chal10 & -1.238 & 159 & .217 & -.156 & -.41 & .09 \\
\hline Chal11 & 4.856 & 158 & .000 & .572 & .34 & .81 \\
\hline Chal12 & 5.499 & 158 & .000 & .616 & .39 & .84 \\
\hline Chal13 & 2.949 & 158 & .004 & .327 & .11 & .55 \\
\hline Chal14 & 2.642 & 158 & .009 & .308 & .08 & .54 \\
\hline Chal15 & 3.379 & 158 & .001 & .377 & .16 & .60 \\
\hline Chal16 & -1.285 & 158 & .201 & -.170 & -.43 & .09 \\
\hline Chal17 & 4.870 & 158 & .000 & .516 & .31 & .72 \\
\hline Chal18 & .779 & 158 & .437 & .088 & -.14 & .31 \\
\hline Chal19 & 1.625 & 155 & .106 & .179 & -.04 & .40 \\
\hline
\end{tabular}

As done in Section 6.2.2.4 for ranking the change items, we also performed pair-wise t-test to determine whether the means of the successively occurring pairs of challenge items are significantly different from each other. We did that only for the items that passed the one sample t-test (as shown in boldface in Table 6.25). The results of the pairwise t-test of the successively occurring boldfaced challenge items in Table 6.25 show that except the pair Chal1-Chal9, all the other pairs of challenge items do not have a statistically significant difference of their means. Therefore, although we have ranked the challenge items in the order as shown in Table 6.25 purely on the basis of their mean values, we should note that there is no statistical significance of the ordering of these 149 
challenges. In other words, although we can infer that out of the 19 challenges, 11 of them are statistically significant, we cannot really rank them in a statistically significant order. This statement excepts the Chal1-Chal9 pair. In other words, upper management resistance was found to be statistically significantly greater than the resistance from developers.

Table 6.25: Research Question 3: Ranking of the Challenges

\begin{tabular}{|c|c|c|c|c|}
\hline Rank & Variables & $\mathbf{N}$ & Mean & $\begin{array}{c}\text { Std. } \\
\text { Deviation }\end{array}$ \\
\hline 1 & Chal9 & 161 & 4.10 & 1.46 \\
\hline 2 & Chal1 & 161 & 3.81 & 1.24 \\
\hline 3 & Chal4 & 161 & 3.72 & 1.36 \\
\hline 4 & Chal12 & 159 & 3.62 & 1.41 \\
\hline 5 & Chal11 & 161 & 3.57 & 1.49 \\
\hline 6 & Chal17 & 161 & 3.52 & 1.34 \\
\hline 7 & Chal7 & 161 & 3.50 & 1.23 \\
\hline 8 & Chal15 & 161 & 3.38 & 1.41 \\
\hline 9 & Chal13 & 159 & 3.33 & 1.40 \\
\hline 10 & Chal14 & 161 & 3.31 & 1.47 \\
\hline 11 & Chal2 & 160 & 3.26 & 1.40 \\
\hline 12 & Chal19 & 156 & 3.18 & 1.38 \\
\hline 13 & Chal3 & 159 & 3.13 & 1.41 \\
\hline 14 & Chal18 & 159 & 3.09 & 1.42 \\
\hline 15 & Chal8 & 161 & 3.09 & 1.59 \\
\hline 16 & Chal5 & 161 & 3.05 & 1.16 \\
\hline 17 & Chal6 & 161 & 2.96 & 1.20 \\
\hline 18 & Chal10 & 161 & 2.84 & 1.60 \\
\hline 19 & Chal16 & 161 & 2.83 & 1.67 \\
\hline
\end{tabular}


All the significant challenges (that have passed the one-sample t-test) are highlighted in Table 6.25 from most important to least important. They are stated below ${ }^{31}$ :

- Rank1: Upper management resistance due to factors such as lack of development under contract, and well-defined project charters, project plans and Gantt Charts in agile development.

- Rank2: Resistance from developers to transform from traditional heavyweight process centric development.

- Rank 3: Problems with development teams that are geographically distributed and not colocated in agile development.

- Rank 4: Development process conflicts due to the differences in lifecycle between agile methodologies (which are characterized by test driven design, short, and focused iterations of development), and traditional methodologies (which have heavyweight processes and long iterations).

- Rank 5: Challenges of the agile teams in integrating the development processes and subsystems with teams within the same organization practicing traditional development methodologies.

- Rank 6: Differences in attitudes towards project success between the management practicing traditional and agile methodologies.

\footnotetext{
${ }^{31}$ It should be noted that although we have provided the ranks of these challenge items purely on the basis of their mean values, they do not have statistical difference in their means. This statement excepts the items in Ranks 1 and 2 in the list.
} 
- Rank 7: Overzealousness (excessive enthusiasm) in team members due to perceptions of agility and fast decision making, leading to decision making without much forethought in agile development.

- Rank 8: Differences in performance management approaches in traditional methodologies (which are more contract and milestone driven), and agile methodologies (which are based on incorporating continuous customer feedback into the development lifecycle.)

- Rank 9: Adopting agile methodologies for use in legacy systems, which are more resistant to changes in internal source code.

- Rank 10: Differences in development processes between agile methodologies (which are more informal requirements driven), and traditional methodologies (which are more formal requirements driven).

- Rank 11: Developer perceptions of micromanagement due to close and frequent interaction with management in agile development.

\subsubsection{Challenges Suggested by Respondents}

As in the success factors survey and the changes survey, the respondents were also requested to list up to five additional challenges they believe are important challenges that would be faced while adopting agile software development practices in organizations practicing traditional, plan-driven methodologies. Their detailed responses, as written by the respondents, are listed in Appendix D. However, as before, the challenges below are 
synthesized below by giving my own perspective. As in the previous two surveys, being an optional question, some of the participants responded to it, while some did not.

It should be noted that, as mentioned for the case of success factors or changes, many of the challenge dimensions/categories that we have identified below overlap with those of the ones that we had previously identified, but the individual challenge items within them are different. Therefore, we list all the dimensions even if they were previously identified so that we do not lose any data that we have obtained. However, it should be remembered that what is important for us is the identification of the major dimensions of the challenges. The list of individual challenge items contributing to any of the dimensions that we have identified could be large, and may not restrict to the ones we had in the questionnaire and the ones brought forward through the open-ended questions.

\section{Resistance from team members}

One of the most important challenges suggested by different respondents is the resistance from the team members fearing change. The adoption of agile practices can be treated as a threat by the different developers. This is particularly true for those who are less adaptive to change. It is required by the developers to work with the testers in test driven development which is the common approach taken in agile methodologies. This can prove to be resistive by both the developers and the testers.

\section{Management resistance}

Resistance can arise from middle level management as well. Managers used with traditional development approaches are used with fixed scope and budget and are less 
adaptive to change. It is also suggested that it can also be challenging for having a good and flexible project manager.

\section{Customer involvement}

It is suggested by some of the respondents that keeping the customers frequently engaged as opposed to their involvement after a long duration (after a few months or after a release) can be challenging. It is also challenging to obtain the buy-in of the customers for agile practices.

\section{Differences in management attitude towards success}

Managers often look for demonstration of success in the form of the budget expended, time spent, and the returns obtained. Managers used with traditional software development approaches often perceive agile practices as "something "new" and needs to be "tried out" first'.

\section{Doing away with documentation}

It is suggested by some of the respondents that people tend to feel secure with having a good set of documentations. Less emphasis on documentations or a lack of it can make people insecure.

\section{Decrease in productivity during transition}

Some people feel there is an added responsibility associated with agile methodologies. They feel that the productivity can decrease due to this. This can be challenging.

\section{Differences in development processes}

Differences in requirements management processes is suggested to be challenging. It is also suggested that it is challenging to have developers accept test driven development. 
Customers; who are used to aceepting the delivery of "almost-ready" code; can prove to be resistive to the development processes associated with agile methodologies.

\section{Differences in performance measurements and project status updates}

In agile practices, it can be challenging to communicate the status of projects because there is often qualitative control and a lack of metric.

\section{Variability in team dynamics}

The variability of the team dynamics can be challenging. Different people have different opinions regarding the functioning of the teams. It is mentioned by one of the respondents that while some people might be pushing forward, others might be resisting backwards. Also, it can be challenging to identify whether it is essential for a particular team member to accompany another, as is often required for pair programming.

The above categories of challenges reveal that they capture most of the important dimensions already considered through the closed-ended questions. It should be noted that the list of challenges already considered as part of the closed-ended questions were prepared based on literature review and review of plenty of experience reports. Therefore, it is not surprising that most of the important dimensions of challenges were already captured.

\subsubsection{Discussions}

We have seen that solely from the analysis of the quantitative data obtained, eleven out of nineteen challenge dimensions that we considered show statistically significant results. The most important of all challenge dimensions is the upper management resistance. 
Challenges with respect to upper management resistance are followed by the developer resistance, problems with geographically distributed teams, differences in lifecycle, variability in subsystems and teams, differences in management attitude towards project success, overzealousness of teams, differences in performance management approaches, adopting agile in legacy systems, and differences in development processes, in decreasing order of their importance ${ }^{32}$. The challenges such as problems with selecting the right agile methodology, developer perceptions of freedom, problems with team size scalability, tester resistance, productivity differences between team members, decrease in productivity during transition, human resources resistance, and conformance with traditional process standards were not found to be statistically significant challenges based on the survey ratings. In the absence of the availability of any previous literature that ranks or prioritizes the different challenge items, we were not able to validate the results that we have obtained with the findings from the literature. However, in Chapter 7, we use qualitative logical reasoning and citations from the literature to support the reasonings/justifications we make in favor or against the observations we make. Further statements of support from the literature, in addition to the ones already given in Chapter 4 , are given in an attempt to establish the results we have obtained.

As we will see in Chapter 7, in the absence of any particular literature justifying our results, based on the discussions from the existing pieces of literature in this area, and the results obtained from the analysis of the qualitative responses obtained through the openended questions, we find that all the eleven challenge categories that have been found to

\footnotetext{
${ }^{32}$ This ordering is purely on the basis of their mean values.
}

156 
be statistically significant are justifiable. However, as we will discuss in Chapter 7, the statistical non-inclusion of all the rest of the eight challenge items in the list of significant challenges is not justifiable in all cases using the logical reasonings and the literature evidences. We call for their further detailed investigation using other research methodologies such as case studies.

Further detailed discussions attempting to justify the results obtained from the data analysis for this research question can be found in Chapter 7. In the interest of not being repetitive in our discussions regarding these, we do not address them any further in this Chapter. 


\section{Chapter 7 \\ Discussions}

\subsection{Perspective}

This Chapter reviews the results shown in Chapter 6, and analyzes them from the perspective of the research goals that were set at the outset. This research started with the following three primary goals:

[Goal 1] Identifying the factors that lead to the success of software development projects that want to adopt agile software development practices

[Goal 2] Identifying the important changes required for adopting agile software development practices in projects practicing traditional plan-driven software development, and ranking them according to their level of importance.

[Goal 3] Identifying the critical challenges/risks that projects may encounter for adopting agile software development practices in organizations practicing traditional lifecycle-based software development, and ranking them according to their level of importance.

To achieve these goals, theoretical frameworks were developed based on what is available in the literature. Different hypotheses, which were subjected to validation through findings from a survey of different agile software development practitioners, were constructed. Many interesting findings have emerged in this process. In this Chapter each of these findings are discussed. They are analyzed from different perspectives such 
as those that are stated in the literature and those that are available from the qualitative responses from the respondents. Further statements of support from the literature, in addition to the ones already given in Chapter 3 and Chapter 4, are given in an attempt to establish/justify the results we have obtained.

\subsection{Success Factors}

On the basis of data analysis, we found that nine out of fourteen hypothesized factors have significant relationship with Success, whereas five do not. The nine factors that showed significant relationship are: customer satisfaction, customer collaboration, customer commitment, decision time, corporate culture, personal characteristics, societal culture, and training and learning. The five that did not show significant relationship are team distribution, team size, planning, technical competency, and communication and negotiation. This led us to revise the a priori theoretical model we had developed in Chapter 3. The revised model is shown in Figure 7.1. 


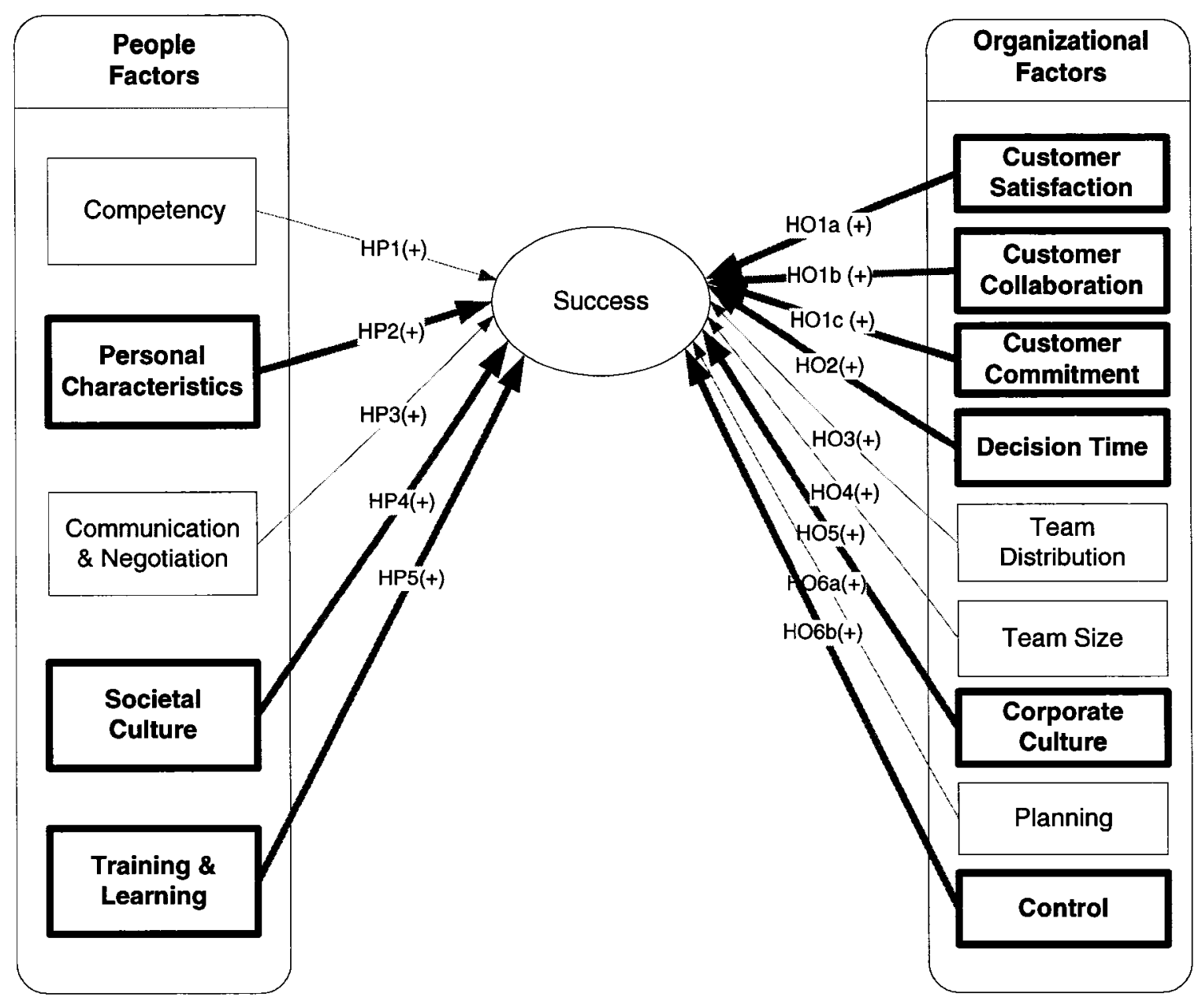

Figure 7.1: Revised Success Factors Framework on the Basis of Survey Data

Let us revisit all the hypothesized factors.

\section{Customer centric issues}

It is worth noting that all the three hypothesized customer centric issues viz., customer satisfaction, customer collaboration, and customer commitment were found to have a significant relationship with Success.

Although solely from the correlation significance point of view all of these three customer centric issues were found to have a significant relationship, by looking at their 
coefficients of correlation it can be seen that customer commitment was found to have a strongest relationship of all, followed by the customer collaboration, and customer satisfaction respectively. This relative behavior is observed solely based on the analysis of the data obtained, and there is no apparent logical justification or literature support that can be provided in support of or against this behavior. It is worthy of further investigation using other means. In an absolute sense, customer commitment and customer collaboration are undoubtedly very important from the point of view of the agile practitioners. It is, however, interesting to observe that while customer collaboration and customer commitment were at least somewhat agreed to be practiced in approximately $78 \%$ and $71 \%$ percent of cases, customer satisfaction was at least somewhat agreed to be practiced in approximately $97 \%$ of the cases. This is, again, not surprising because irrespective of what approach the project teams take, it is likely that most people would agree that they try to satisfy their customers.

Viewing it from what the different respondents have suggested as other success factors in the open-ended questions, almost all of them suggested in different forms that customer commitment, and customer collaboration were important success factors. There were no significant additional suggestions in favor of the customer commitment factors. Thus the results of the open-ended questions are also intrinsically suggestive of the customer commitment and collaboration in different morphs. Development teams want the customers to remain closely associated, engaged, and committed to the project. They do not want buying software to be viewed by the customers as similar to buying commodities such as groceries! Thus the results of the analysis of the suggestions 
obtained from the open-ended questions are also in conformance with those obtained from the analysis of the data obtained from the closed-ended question.

The whole idea of agility is trying to develop software efficiently so as to satisfy the customers. This requires that the customers are committed on the project, and they involve/engage themselves closely with the development team. It is worth recalling that customer commitment was even found to be one of the four predictors of the consolidated dependent variable Success in its regression model measured with the help of the five measures mentioned in Chapter 3, whereas customer collaboration was found to be a predictor of the increased return on investment taken alone as a measure of Success.

In conclusion, it can be said that it is sensible that we found significant relationship between Success and customer centric issues. However, it is contextual to warn that there may be practical challenges trying to engage customers. In an interesting article (Deias et al., 2002), the authors describe their experiences of introducing the agile methodology $\mathrm{XP}$ in a start-up company, and found that there were several challenges from the customers while involving customers and collaborating with them. For example, they mention:

"Our customers do not like contractual provisions that address the risk of changing requirements. Most of our customers regard software as just one of the goods that they acquire, and want to buy it using the same kind of contractual agreements used for buying other products. ... The fear of the business people is that by opening up the development process to our 
customers, we will end up having customer going around shopping for specific people, trying to build the team of their choice for specific projects ...".

There are also other practical challenges of having customers on-site that have been brought forward by them, that should be addressed.

Jensen (2002) reports his experience from several projects that for having success in a project, cooperation between users (customers) and developers is very important. The importance of customer commitment, collaboration, and involvement is also evident from the findings of Graffin (2001) from implementing an agile methodology in an organization. Our findings regarding the importance of such customer centric issues as important success factors is further strengthened by the findings from such pieces of literature.

\section{Decision time}

The factor, decision time, also demonstrated significant correlation with Success. The coefficient of correlation also shows that the strength of the relationship is relatively high as compared to the rest of the fourteen factors. These results are not surprising considering that the whole idea behind agility is being fast. Fast communication, informal communication, effective communication, which are cited by many researchers such as, Turner and Boehm (2003) and Ambler (2005b) in different ways enable fast decision making (eWorkshop, 2002), which in turn leads to the success of projects practicing agile software development. This is further evidenced from the fact that $84 \%$ of the agile practitioners that were surveyed agreed that they strove to make important project decisions rapidly and within short timeframes. Timing is an important factor. As seen from the additional success factor responses obtained from the survey participants, 163 
cutting down on the time to delivery and time to market are suggested as important factors. One of the respondents even considered "time boxing" as one of the most important factors. It is suggested that to be successful, it is important to scale down the requirements, and slice the whole time into short durations. The implicit idea behind this is that by doing all of the above, among other advantages, the decisions can be made more rapidly and efficiently. By looking at the responses to the open-ended questions, we feel that a better construct in the hypothesized theoretical framework could have been the consolidated factor "timing issues". However, we based all the constructs in the theoretical framework initially purely based on the surveyed literature, and came up with the "decision time", which captures a singular dimension of the timing issues.

\section{Corporate culture}

The factor, corporate culture, was also found to be statistically significantly correlated with Success. The strength of this relationship was also found out to be high relative to the strengths of the other fourteen factors that were considered in this study.

It is not surprising to see that the responses from the practitioners of agile software development have shown significant relationship with Success, considering the citations from the existing pieces of literature. For instance, Lindvall et al. (2002) have mentioned that:

"To be agile is a cultural thing. If the culture is not right, then the organization cannot be agile".

During the eWorkshop that was held in 2002 (eWorkshop, 2002), most of the experts agreed that the corporate culture was an important success factor. It may be recalled that 
successful agile software development requires that the organizations are dynamic and are fast changing (Abrahamson et al., 2002). The organization should have the right culture such as supporting rapid communication, dynamicity in requirements changes, trusting people, and obtaining fast feedback from customers. These were evidenced from the discussions in Lindvall et al. (2002).

Our results are as well in conformance with the findings of Bossavit (2002) in using an agile methodology in two different organizations that there are, indeed, certain cultural values that are necessary. The following statements of the author provides good light into the issue:

“... the most crucial feature of any successful corporate culture must be a willingness to reflect upon itself, to assess whether it needs to change itself, and, if need be, to embark on such changes as it finds necessary".

Corporate culture is an important success factor, indeed. In fact, the statements made by Bossavit (2002) not only reflect upon corporate culture, but also the importance of different personal characteristics. This is not surprising because organizations are made up of people and the characteristics of people are undoubtedly very important to success.

In the survey, the dimension, corporate culture, was captured with the help of the following eight measures:

- Encouraging rapid communication.

- Culture for trusting people.

- Management has the culture for supporting the decisions of the developers. 
- Culture is customer centric.

- Encouragement of fast feedback from customers

- Encouragement of changing requirements.

- Absence of a bureaucratic management structure.

- Management, developers, and testers are in total agreement to use agile practices.

The analysis of the percentage responses obtained against each of these eight measures suggests that the majority of the practitioners of agile software development strongly agree that they have all the above cultural factors in their organizations.

It should be further observed that although corporate culture has not been found during regression analysis to be a significant predictor of the consolidated dependent variable Success (measured with the help of the five measures mentioned in Chapter 3), it was found to be a predictor of the Success measures, increased ability to meet with the current customer requirements, and improved business processes, each taken in isolation as the dependent variable.

Corporate culture, as a success factor, was further evidenced from the responses of the open-ended success factor questions. Many of the additional success factors that were put forward by the different respondents broadly captures the dimension of corporate culture. For instance, the respondents felt that there should be an environment of supportive management, there should be buy-in from all stakeholders (inclusive of management), there should be lack of politics, good customer-vendor relationship in the organization, and adaptability to changes. 


\section{Control}

The factor, control, was found to have a statistically significant relationship with Success. By looking at the correlation coefficient, which indicates its strength of its relationship with Success, it is perhaps the second strongest (the strongest being societal culture, to be discussed shortly) amongst all the fourteen hypothesized factors. We measured this construct by asking respondents whether they practice qualitative control (as against having quantitative performance measures). Of the majority of the respondents, approximately $62 \%$, at least somewhat agreed to practice it.

Anderson (2002) reports a practical experience story with using "morning roll call" (daily standup) in having project success. Such a report supports our finding that qualitative control is an important success factor. The observations from statistical analysis are also supportive of the comments made in literature such as Boehm and Turner (2003), Cockburn (2002), Cohn and Ford (2003), McMahon (2004). Their observations and comments are also implicitly or explicitly suggestive of qualitative control.

Not only from the correlation analysis results, but also by observing the regression model for the consolidated dependent variables Success (measured with the help of the five dimensions mentioned in Chapter 3), we see that it emerges as one of the four predictors of Success. However, in the regression models corresponding to each of the five dependent variables taken in isolation, it appears as a predictor only for increased return on investment, and it does not appear as a predictor for reduced delivery schedules, increased ability to meet with the current customer requirements, increased flexibility to 
meet with the changing customer requirements, and improved business processes. This indicates that control has a relatively strong influence on increasing the return on investment. Such a behavior is only observed from analyzing the data obtained from the closed-ended questions. Our literature survey cannot justify this observation either in favor or against. It is worthy of validation through further studies.

Analysis of the responses obtained through the open-ended success factor questions also suggest different qualitative ways of demonstrating project progress such as weekly progress showcases to the customers, and/or daily standups. Interestingly, one of the respondents also suggested showing progress in terms of "completed story points per iteration".

In sum, as has been evidenced from statistical analysis, qualitative analysis of the open-ended responses, and the existing literature, qualitative control emerged to be a significant success factor.

\section{Personal characteristics}

Although personal characteristics were also found to have a significant relationship with Success, the strength of its relationship relative to the other hypothesized factors is not commendable. This observation is somewhat surprising when the existing literature is revisited.

According to Alistair Cockburn (eWorkshop, 2002), one of the pioneers and prominent figures of agile software development, "Good people are key to success with big teams". The participants of the eWorkshop that was held in 2002 (eWorkshop, 2002) were supportive of personal characteristics such as having honesty, collaborative attitude, 
sense of responsibility, readiness to learn, and work with others as important success factors. Technical expertise and professional qualifications alone cannot succeed a project. The importance of personal characteristics such as cooperativeness is also reported in the literature (Jensen, 2002). Similar observations suggestive of the above facts can be made by reviewing the following pieces of literature: Ambler, 2005c; Boehm and Turner, 2003; Boehm and Turner, 2005; Cockburn and Highsmith, 2001 and Cockburn, 2002. They were already discussed in Chapter 3.

The construct, personal characteristics, was measured with the help of the following dimensions:

- Having majority of the members with strong interpersonal and communication skills.

- Having majority of the people who are honest.

- Having majority of the people who are motivated.

- Having majority of the people who have collaborative attitude.

- Having majority of the team members consisting of people who have sense of responsibility.

- Having majority of the team members consisting of people who have readiness to learn.

The majority of the respondents at least somewhat agreed (in fact, most of them strongly agreed) to have team members having such kind of personal characteristics. However, it did not show a strong relationship with Success compared to the other 169 
factors. However, a number of responses to the open-ended success factor questions were suggestive of personal characteristics as an important success factor. They are as follows: Having intellect; Taking up responsibility; Having a base of good principles; Willingness of programmers to work as pairs; Passionate; Not being a perfectionist; Self-criticism; Courage to tell the truth about project; Respect of individual; Good People; and Humility.

Although it is not to be denied that the construct personal characteristics does show that its relationship with success is significant, we expected that the relationship would be stronger than what we obtained. This observation is contrary to earlier research that is supportive of the role of personal characteristics. Therefore, further studies using other methodologies should be warranted to closely observe this observed phenomenon.

\section{Societal culture}

In this study, the societal culture was measured with the help of the following four dimensions:

- Having people in the society who are in general communicative.

- Having people in the society who are in general dynamic.

- Having people in the society who are in general have progressive attitude.

- Having majority of the members of the team with similar social culture, even though they might be belonging to different nationalities and provinces.

The consolidated percentage figures for the responses obtained suggest that a majority of the surveyed team members have somewhat agreed to possess or abide by the above dimensions of societal culture. 
Societal culture was observed to have a significant relationship with Success suggesting that having people in the society who are communicative, dynamic, progressive in attitude, and having team members with similar social culture, taken together, are important success factors. The correlation analysis results also demonstrate a relatively very strong coefficient of correlation (in fact, the strongest of all), thereby being strongly suggestive of the presumption based on observations from the existing literature that societal culture does play a significant role as a success factor.

It should be noted that multiple regression analysis suggests that societal culture was found to be a significant predictor of the consolidated Success variable amongst all the fourteen factors considered together. Even when regression models were constructed with each of the five measures of Success (see Chapter 3) taken in isolation, it was observed that societal culture is a significant predictor of the return on investment. This observation is obtained purely from the data obtained from the closed-ended questions, and cannot be supported with the existing literature that were surveyed.

Interestingly, the additional success factor responses obtained from the respondents against the open-ended questions, do not reveal any additional dimensions of societal culture.

\section{Training and learning}

The construct, training and learning, was found to have a statistically significant correlation with Success. Furthermore, it was also found to have a relatively strong relationship. These results are not surprising because there is a strong evidence in the 
existing literature (Boehm and Turner, 2005; Drobka et al., 2004; eWorkshop, 2002, Lindvall et al., 2002) which is suggestive of training and learning as a success factor.

This construct was measured with the determination of the willingness to continuously learn from one another and train the team members through mentoring and professionally guided discussions than through formal training. Thus the emphasis is on continuous, informal learning. The results showing that the majority of the respondents strongly agree that they practice this approach of training and learning in their teams. The results showing significant and relatively strong correlation with Success imply that the majority of the agile practitioners that were surveyed believed that continuous, informal learning (over formal training) was a significant success factor. Informal learning helps in knowledge diffusion in an organization. However, it should be noted that there are authors such as Dingsoyr and Hansen (2004) who believe that informal learning methodologies such as pair programming alone cannot be effective means of knowledge diffusion in an organization. Dingsoyr and Hansen (2004) have suggested postmortem reviews as a lightweight mechanism to aid in such a purpose.

The regression model for Success also shows training and learning to be a significant predictor of Success. Even the results of the regression modeling with each of the five measures of Success, taken in isolation as a dependent variable, also show the training and learning construct to be a significant predictor of the reduced delivery schedules and increased return on investments.

The above results are further supported by the responses to the open-ended questions, which indicate usage of informal training methods such as pair programming. This will 
enable transfer of knowledge through discussion of designs. However, there were a few respondents who believed in having full time coaches and taking help of outside coaching services to succeed.

\section{Team distribution}

Team distribution was measured with the help of two dimensions:

- Having members in the team that are geographically closely located.

- Having other teams (with which interactions are made) geographically closely located to ours.

The results of the analysis of the closed-ended data obtained from the respondents do not suggest any significant relationship with Success. While the majority of the respondents maintained that they strongly agreed to have geographically closely located members of their own team, they strongly disagreed with the need to have other teams with which they interact geographically closely located to theirs. Thus from the results of the correlation analysis it can be suggested that geographical collocation of own team members or members of other teams with which interactions are made does not show any significant relationship with Success.

The above results are contrary to the suggestions from existing literature for having collocated teams as a success factor (e.g, eWorkshop, 2002; Lindvall et al., 2002). Further, there are earlier authors (Ambler 2005b; Boehm and Turner, 2003; Cockburn, 2001; McMahon, 2004 and Turner and Boehm, 2003) who have emphasized on close proximity, and communication issues such as face-to-face communication. Our findings 
are also not in conformance with the suggestions made by Turk et al. (2002), in which they have mentioned the identification of limited support for distributed development of agile processes as one of the important factors in agile development. In other words, team distribution should be an important success factor. In this context, it is worth citing the experience of Motorola, Inc., in their global development projects. Copeland (2001) mentions that Motorola, Inc. had used an agile methodology, XP, in some of their development projects, and found that it was not suitable for their global development projects. The reason cited relates to the fact that XP encourages working in small teams, and they had difficulty doing so in their global development projects. This is further suggestive of the fact that team distribution should be an important factor in determining success.

The apparent inconsistencies with the literature that we have obtained through the analysis of the data obtained from the closed-ended questions could be attributed to the differences in measures of success that we considered in this study, with the ones that are suggested by the authors. The suggestions to the open-ended questions from the respondents are not suggestive of any significant success factors that can be attributed to this dimension. This behavior could be partially justified by the fact that may be a significant number of respondents would not justify team distribution, or even communication and negotiation, as a major success factor as compared to many of the others that we considered. Let us take the example of the case reported by Fowler (2006) in which he reported success in using agile practices in offshore development project, in spite of the fact that in offshore development environments communication and 
negotiation would pose major challenges. Fowler (2006) mentioned some strategies which could successfully overcome the challenge of having distributed teams.

Thus, because it is hard to justify the results we have obtained, team distribution is a candidate for further detailed investigation using other research methodologies such as case studies.

\section{Team size}

The construct, team size, failed to demonstrate a significant correlation with Success. Using this construct it was measured whether the respondents worked in small teams. This is contrary to the popular belief in the literature that we surveyed. Ambler (2005b), among others, emphasize on close face-to-face communication. Having small teams was suggested in the literature (eWorkshop, 2002, Dyba, 2000). Among the suggestions obtained from the eWorkshop participants (eWorkshop, 2002), Frank Maurer suggested small teams in university setting, Ken Auer said 12 using pure XP, Tim Mackinnon provided different figures (corresponding to different situations) for team size, but all of his suggestions are for a team size less than 25 . There were other figures ranging in hundreds were also proposed. The rejection of small team size could be connected with these suggestions because we have surveyed different types of people working in different project types. This is in conformance with the following statement ${ }^{33}$ of Randy Miller in the eWorkshop (eWorkshop, 2002):

\footnotetext{
${ }^{33}$ The exact form in which this statement and the one below for Alistair Cockburn were made might be slightly different from what is stated here. The quotation here is taken verbatim from the eWorkshop(2002).
} 
“... (team) size is not an issue specific to agile development. It's a best practice for any type of development to break into smaller teams."

The reason, however, why we hypothesized small team size to be a success factor is, logically, and as mentioned in some of the above mentioned pieces of literature (see the discussions on team size in the eWorkshop (2002)), having a small team size enables effective communication, fast feedback, and prompt decision making. Our intuition is in conformance with the suggestion of statement of Alistair Cockburn in the eWorkshop (eWorkshop, 2002), which reads:

“... (team) size is an issue. As size grows, coordinating interfaces becomes a dominant issue. Agile with face-to-face breaks down (becomes more difficult/complex) past 20-40 people (interface becomes an issue)."

Our findings are also inconsistent with the Turk et al. (2002) who have identified that limited support for development involving large teams in agile processes as one of the challenges in agile development. Also Parrish et al. (2004) in their early study found that productivity influences of pair programming as the team/organization size increases. Since pair programming is a common practice in agile software development, this gives us another clue that team size should be an important factor.

Of course there are other instances reported in the literature of using agile practices in large team environments. Pine (2001) mentions his positive experience of successfully using XP (a methodology following the agile practices) in a large, multi-team environment. Of course, whether he received success according to our definition cannot be inferred from his paper. The instance of Harrisson (2003) is also worth noting. They 
have reported of positive experiences using agile practices in large projects that have large number of teams, and large team size. All these stories of different opinions may also partially help us justify our observation from data analysis that team size or project size may not be a success factor.

Because of the varying thoughts, opinions, arguments, and justifications regarding this issue, we call for further research to investigate this issue in detail. In particular, we suggest that different projects with varying team size should be investigated in detail preferably using a case study based approach, which might be helpful in making detailed observations regarding this issue.

\section{Planning}

Planning was not found to have a significant relationship with Success. The aspect of planning that we measured is internalized, informal, and undocumented plans (as against formal plans). Insignificant relationship with Success is contrary with the literature we surveyed which suggest internalized planning over the formal ones (Boehm and Turner, 2003; Cockburn, 2002; Cohn and Ford, 2003; McMahon, 2004). This indicates that formal planning cannot be discarded from consideration. Although, by abiding by the literature, we hypothesized for internalized undocumented planning, and against formal planning, it being not a success factor is not counter-intuitive. Agile practices are relatively new, most experienced people we surveyed have been working in traditional type projects for years, and it is unlikely that by surveying such kind of people we will get its strong relationship with Success. Furthermore, many of these people work in complex projects, involving a large number of personnel, and in big organizations, which 


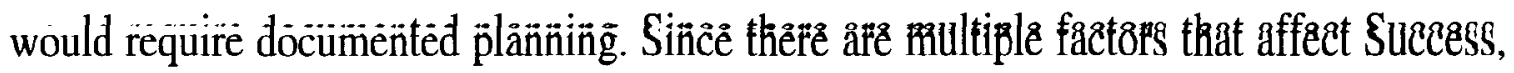
intuitively, there can be Success in a project (attributable to other factors), even when formal planning was undertaken. The above statements could possibly justify the results we have obtained through data analysis of responses to the closed-ended questions for the possibility of "planning" as a success factor.

\section{Technical competency}

It was found out through analysis of data from closed-ended questions that technical competency does not have a statistically significant correlation with Success. The majority of the people that we surveyed at least somewhat agreed that they have technically competent people. However, no significant relationship was found between technical competency and Success. First, this is counter-intuitive. This is because, logically, having competent or incompetent people should make a difference to the success of a project. Secondly, our literature survey (Cohn and Ford, 2003; Boehm and Turner, 2005; and Cockburn and Highsmith, 2001) is also implicitly or explicitly suggestive of the competency dimension. Also, this is contrary to Barry Boehm's suggested principle of using fewer and better people (Boehm, 1981).

In regards to competency as a success factor, the practical experience of Deias et al. (2002) introducing agile in a start-up Internet company is also worth noting. According to them:

"If the team lacks the necessary programming experience results will be at best marginally better than what one would expect from any other methodology". 
Our initial hypothesis of having competency as a success factor is also not in conformance with the observations of Deias et al. (2002). Experience and competency are very important.

In addition to the citations from the literature, when the respondents were surveyed through open-ended questions for additional success factors, there were a number of them who suggested different factors that are suggestive of different competencies such as the following: Talent; Developers know the business and can sometimes be their own customers; Smart people; Individual capabilities of team members; Craftsmanship; Developers' experience; Domain knowledge; Developer familiarity with the technical platform; and Developer skills.

In view of the above discussions, we are unable to come to any conclusions regarding the insignificant relationship of the competency construct with success. We see that many respondents have made many suggestions in favor of different technical and nontechnical competencies. Thus, we call upon further investigation regarding this issue.

\section{Communication and negotiation}

The construct, communication and negotiation, was measured with the help of the following dimensions:

- Having mechanisms that enable personnel to communicate and negotiate quickly and effectively with developers, operations, support, customers, management, and business areas.

- Having face-to-face communication and negotiation. 
- Communication and negotiation between people who are physically close to one another.

- Communication and negotiation between people who work in the same (similar) time zone.

- Having people in projects that are amicable to each other to such an extent that they communicate with each other with trust and good will.

Communication and negotiation was also found not to have a significant relationship with Success, based on the survey data obtained through the closed-ended questions. This is, perhaps, the most surprising of all the above factors that were found not to have a significant relationship with Success.

Several previous pieces of literature support effective communication mechanisms to be in place for success. Some of them that are implicitly or explicitly suggestive of the same include Ambler (2005b), Cockburn (2001), eWorkshop (2002), Lindvall et al. (2002), Turner and Boehm (2003), and McMahon (2004). Logically, as well, fast and effective communication supports fast decision making, adapting with the dynamicity of projects, and fast knowledge transfer between team members in a project.

Considering the points of view raised through the additional open-ended success factor questions, as well, we see that a number of factors such as open and continuous feedback between team members, using simple but effective communication tools, using information radiators such as charts and cards on the walls, and using communication rules that are suggestive of communication as a success factor were raised. 
As both previous literature and our logical justification are not in consistence with the results of the data analysis that communication and negotiation do not have any significant relationship with success, we call for further studies in this domain to be able to draw any conclusive inference.

\subsection{Changes Required}

In Chapter 6, we had seen the statistical ranking of the change items based on the data obtained. Since our literature search did not reveal any study that ranks or prioritizes the different change items, we will not be able to validate the results that we have obtained with findings from existing literature. However, we will use qualitative logical reasoning and citations from the literature to support our reasonings/justifications of the observations.

We considered four categories of changes, namely:

1. Changes in organization culture

2. Changes in management style

3. Changes in knowledge management strategies

4. Changes in development processes

Based on the results we have obtained, we have seen that the most important of all changes is the changes in the management style, followed by, in decreasing order of importance, the changes in organization culture, changes in development processes, and changes in knowledge management strategies. Our results from data analysis lead us to believe that although one change category is more important than another; all of them are statistically significant (passed the one-sided t-test). This is consistent with our literature 
survey finding that all the four change categories we considered are important (of course, as just said, one is more important than another).

Logically, from our perspective, of all the four categories of changes, changes in management style, and the changes in development processes are probably the most important of all changes that are required. The agile approaches use lightweight processes. They encourage individuals and interactions over processes and tools, responding to changes over following plans, and paying more emphasis on collaborating with customers over negotiating contracts with them, and providing working software over providing a comprehensive documentation (Fowler and Highsmith, 2001; Fowler, 2002).

\section{Changes in management style}

The discussions in the last section showed that in agile approaches there is a strong emphasis required on shifting the style of management style. The management in organizations that have been used to traditional, plan-driven approaches to software development for long are often the most resistive. As one of the respondents pointed out:

"(there is) management desire for fixed price, fixed scope".

This type of management attitude for fixed scope and fixed price is resistive to change and adaptation, which are important for agile approaches. As long as the management does not change ${ }^{34}$, the development processes cannot change, the culture cannot change, or the knowledge management strategies cannot change. Thus it is intuitive that changes in management attitude are the most important of all the four

\footnotetext{
${ }^{34}$ Obviously, in practical situations, it is never the case that one has to take place before the other can happen. Such justifications are made in order to assess the relative importance of each other.
} 
categories of changes. As has been indicated in the previous pieces of literature such as Boehm and Turner (2005), Cohn and Ford (2003), McMahon (2005), and Nerur et al. (2005), management styles must change from command-and-control management to leadership-and-collaboration, from authoritative to collaborative and pluralist decision making.

Based on the results of the data obtained through responses to the closed ended questions, the responses to the open-ended questions, and the citations available in the literature, it is suggested that for becoming agile, the most important thing would be that the management in organizations adopting agile practices collaborate with the rest of the development team members, work closely with them, seek their suggestions, remain open about the status of their projects with the rest of the team members, perform small-scale scope management, without, however, micromanaging the developers, accept uncertainty and risk, and remain open to changes as might be required by the customers or developers for developing a quality product. Management who are traditionally habituated to drawing up well-defined plans, negotiating contracts with the customers, following them, and sticking to them would need to change their style of management. The role of the management should be to facilitate the overall development activities without commanding and controlling the development team and charging them for work they have done in their intention for developing a quality product. 


\section{Changes in organization culture}

Having justified the importance of management style amongst all the types of changes, let us now examine the remaining categories of changes, i.e., the changes in the organization culture, the changes in development processes, and the development in the knowledge management strategies. The issue of knowledge management is clearly the least important amongst all the three above, because the issue of knowledge management arises only when the development has started. The development processes required in agile development cannot sustain if there is no suitable organization culture.

Changes in culture that are required include culture to work in teams rather than developing solitarily with individually assigned roles, culture which considers satisfying customers as the most important and doing all development centering around that goal, culture which provides freedom to the developers for choosing which modules to develop and how to develop. In addition to the results that we have obtained from data analysis, the discussions in the existing pieces of literature such as Boehm and Turner (2005), and Nerur et al. (2005) are also in conformance with these ideas.

\section{Remaining changes}

Having discussed the importance of the changes in management style, and organization culture, the remaining change categories are the changes in the development processes, and the changes in the knowledge management strategies. Clearly, the issue of changes in knowledge management strategies is secondary relative to the changes in the development processes.

Of all the changes in development processes, the most important changes that are required are shifting from heavyweight process centric development in organizations 184 
practicing traditional, plan-driven development. As has been seen earlier, the agile approaches require close interactions between individual team members themselves and between the team members and the customers, responding to change, having a working software rather than following fixed plans, processes, and tools, and documenting them. The agile approaches encourage development in short iterations (time frames) that are driven by (automated) test plans. The development processes in agile approaches part away from following traditional lifecycles such as the Boehm's spiral model discussed in Chapter 2. The development in agile approaches is evolutionary meaning that a small piece of functionality is developed first, and then it is revised incrementally by collaborating with the customers. The last important change that is required in the development processes is to part away from development guided by standard contracts, and quantitative measures of success. While it is difficult to justify (logically or with the help of existing literature) the order of importance of the above change items that are obtained from the closed-ended survey results, all the above changes in development processes are considered important and are cited in the exiting pieces of literature such as Nerur et al. (2005), and Boehm and Turner (2003).

The above observations are also in conformance with the large number of suggestions received against the open-ended questions for changing the development processes such as follow test-driven development, develop in small steps, and build incrementally and continuously, program in pairs, and develop functional tests in conjunction with writing the code.

Finally, changes in how knowledge is managed are important. But the results of data analysis, the results of the analysis of open-ended questions, and the logical reasoning 185 
seen above ranks it last amongst all categories of changes. Since the agile approaches lay more importance to delivering working software over documenting, the tacit knowledge should be captured and managed in agile approaches, so that valuable information is not lost and/or successes are pursued, and failures are not repeated. However, it is pertinent to mention in this context that, as pointed out in a discussion in an earlier section, there are authors such as Dingsoyr and Hansen (2004) who believe that informal learning methodologies such as pair programming alone cannot be effective means of knowledge diffusion and management in an organization. Dingsoyr and Hansen (2004) have suggested lightweight knowledge management mechanism to aid in such a purpose. As pointed out by one of the respondents in an open-ended question only that knowledge should be captured that will help the customers.

\subsection{Challenges Involved}

Nineteen challenges were hypothesized from the literature and were ranked. As in the case of the changes required, our literature search does not reveal any ranking of different challenges. We, therefore, try to justify below the findings from data analysis with the help of logical justifications and giving citations from literature whenever appropriate and feasible. These justifications apply to challenge item rankings obtained purely on the basis of their mean values. It should recalled that most of the subsequently occurring significant challenge items were not found to have a significant difference of their means.

Resistance is the biggest challenge in any transformation. In our study, we considered resistance from four classes of employees:

\section{- Developers}




\section{- Testers}

\section{- Upper management}

- Human resources

Of all these employees, the results of the analysis of data obtained from the closedended questions reveal that the resistance from the upper management is most challenging, followed by resistance from developers, and insignificant from testers and human resources. These results are very much intuitive. As we have seen in the earlier section, management, whether it is upper management or middle management ${ }^{35}$, is probably the most resistive of all. Managers used to develop software using traditional, plan-driven approaches tend to favor "fixed price, fixed scope" approaches. They want to demonstrate success in progress. They are much less tolerant of risks of failure in undertaking new approaches, more so for the ones that are new, unproven, and do not have adequate track records of success. In this context, it is also worth citing the article by Copeland (2001). Copeland (2001) makes citations about the findings of James Knox, an Ottawa-based independent consultant, who observed that it was difficult to introduce XP (a particular type of methodology following the agile philosophy), because he received no support from management at all. This further strengthens our finding that management resistance is a very important challenge in agile development. Having said that, it was also worth mentioning that there are exceptions, and ways of overcoming management resistance. Different people can suggest different ways of overcoming

\footnotetext{
35 Resistance from middle management was suggested, in addition to the resistance from upper management, in the responses to the open ended questions. However, it did not show up in the initial set of
} 
resistance. Let us take the example of pair programming. Pair programming is an often used programming technique used in agile software development. In general, as seen earlier, management, who are especially resistive of paying two programmers to do the same work. However, Williams and Kessler (2002) provides their positive experience on how to successfully overcome such a management resistance.

Compared to the resistance from managers, the resistance from developers is relatively less challenging, because the developers often do not see the large scope of the projects, they develop small functionalities that they are asked to. It is intuitive and convincing that if the management is convinced and supportive of adopting agile practices, a major challenge can be overcome. Of course, there are still other challenges that come from developers as resistance to adopting agile practices such as resistance to learning and using new way of developing software, and using tools that they are not familiar with, laying lesser emphasis on producing artifacts that do not accompany the final desired deliverable - the working software. Having on-site customers in development can also be challenging and resistive ${ }^{36}$ (Deursen, 2001; Thorup and Jensen, 2003). Thorup and Jensen (2003) share their thoughts of how customer-developer collaboration can be improved. Such and other challenges are further evident from existing pieces of literature such as Cohn and Ford (2003), Highsmith (2000), and Nerur et al. (2005). Furthermore, there are

challenges we considered, because they were collected based on the existing pieces of literature that we surveyed.

${ }^{36}$ Although there can be developer resistances to working with customers, there are success stories available as well. For instance, Crispin (2005) reports a successful experience of involving customers in test-driven development. In her experience report, she mentions of having customers to drive the testdriven development processes. Further, since working with customers can be resistive, Farrell et al. (2002) shares their practical experiences and recommendations as how best to improve customer-developer collaboration. Their recommendations include having customers and developers work as a single team, stories be owned by customers, and having improved mechanisms for tracking and steering. 
additional challenges that are obtained through the open-ended questions and that can be attributed to developer resistance. Those are further supportive of the importance of developer resistance to accepting agile practices.

Much less (in fact, statistically insignificant) importance is shown by the results of data analysis for tester resistance and human resource resistance to adopting agile practices (Cohn and Ford, 2003). In general, testers should not be much resistive to agile practices because they test what they are asked to. The resistance of the testers should not be an extremely challenging issue. The resistance of testers can arise from the fact that some testers who are used to the traditional idea of testing after coding might be resistive of testing while coding (a common approach used in agile methodologies). Therefore, the lesser ranking that is obtained for tester resistance as compared to the management and developer resistance is intuitive. Existing surveyed literature also do as not evidence reports are substantially contrary to this claim. In fact, the additional responses obtained through the open-ended questions do not depict any interesting challenges that can be related to tester resistance.

Human resource resistance does not show as an important statistically significant challenge as well. This is also intuitive because in most cases human resource personnel do not bother about what development methodologies and processes are undertaken by the teams. However, having said that, it should be clarified that this was considered as a challenge item by following the statements mentioned in Cohn and Ford (2003), which suggested that the concepts of work sharing and pair programming are often resisted by the human resource teams in traditional organizations because in such cases it becomes 
difficult for them to track progress of each team member. However, we feel that in most practical situations the reality might be different. The progress reports of the individuals come from the development team managers. The human resource professionals do not come to see the periodic progress of employees.

In addition to the above discussions surrounding potential resistance from different stakeholders mentioned earlier, it should be mentioned that our results regarding the important resistances are also consistent with the findings of Graffin (2001), obtained while implementing an agile methodology in an organization, that buy-in from all key participants is very important prior to an agile methodology's implementation.

Other than the most challenging resistances from management and developers to adopting agile practices, the next major challenge obtained from the results of the data analysis is problems with distributed teams. This is, indeed, an important issue. The agile approaches call for close interactions, and feedback between team members. Having close interactions becomes challenging with geographically distributed teams. The existing literature (Cohn and Ford, 2003) is also supportive of this fact. Working in distributed teams decreases the pace of communication, and decision making required in agile approaches. Thus the relatively high importance obtained from data analysis for this type of challenge is not surprising. Our findings are consistent with the findings of Turk et al. (2002), in which they have identified that limited support for distributed development of agile processes is one of the important challenges. Of course, their findings cannot help us rank the level of importance of this challenge. In this context, it is also worth citing the article by Copeland (2001). Copeland (2001) makes citations about 
Motorola, Inc., who have used an agile methodology, XP, in some of their development projects, and have found that it is not suitable for their global development projects. The reason cited relates to the fact that XP encourages working in small teams, and they had difficulty doing so in their global development projects. This further strengthens our finding that distributed development can be a very important challenge in agile development.

The construct, differences in lifecycle, was statistically ranked to be important next to management and developer resistance. This somewhat high rank of this challenge item is not surprising, and it is supportive of the following observation made by Boehm and Turner (2005). Agile practices focus on delivering immediate value to the customers. In contrary to the plan-driven development processes, which are heavyweight having long iterations and delivering value at the end of the complete lifecycle, agile processes are test-driven, and have short, focused frames of development.

Because of the differences in the development processes, there can be significant challenges that might arise in integrating agile processes in one team or subsystem, when the other teams or subsystem with which interactions are made practice traditional development methodologies. However, this is much less challenging compared to the challenges arising due to the changes in development processes in the team itself. This logically justifies the fact that data analysis shows that such integration issues are important challenges.

Overzealousness of teams was ranked relatively higher compared to the other hypothesized challenges. The importance of the challenge arising due to overzealousness 
of teams could be attributed to the observation made by Cohn and Ford (2003) that since the agile practices are characterized by fast decision making, overzealousness might very well lead to making decisions without much forethought.

As seen in Section 7.3, the survey results show that the changes in management style are critical. The reasons were logically justified as well. Though they do not rank the challenges, they have identified this to be an important challenge. The above reasoning also justifies the importance obtained through data analysis of the challenges surrounding the differences of the attitudes towards project success of the management practicing traditional and agile methodologies, and the differences in performance management approaches. In agile approaches the managers do not have fixed plans and performance measures to rely on for demonstrating success. Ideally, the managers do not have any contracts to abide by or milestones to dictate progress. Success in agile approaches is qualitative, and is, generally governed by the continuous satisfaction of the customers.

Considering the case of incorporating agile in legacy systems, although it is a challenge to subject these systems' requirements and their associated processes to rapid changes, such issues are manageable because such situations are unlikely or practically low in number in legacy systems which have been there for years. However, there are instances reported in the literature (e.g., Gawlas, 2003; Rasmusson, 2003) where large, legacy-type, and/or mission-critical systems were done better (or at least done properly) using agile practices. Therefore, the relatively low rank obtained for adopting agile methodologies for use in legacy systems is understandable, because it is likely that there would be varying opinions from respondents regarding this issue. Not everyone would 
consider it a major challenge. Thus, it is comprehensible that it has been ranked not as important challenges as compared to the others mentioned above.

As discussed in Section 7.3, there are vast changes in development processes that take place while transitioning from development using traditional, plan-driven approaches to that using agile. Changes from heavyweight process centric development to those using lightweight processes can be extremely challenging. Close interactions between individual team members themselves and between the team members and the customers, responding to change, parting away from following fixed plans, processes, and tools, and documenting them, development in short iterations (time frames) driven by (automated) test plans are almost a paradigm shift in development approaches in plan-driven development organizations. However, although this is found to be a statistically significant challenge, the logical reasoning expects it to have been ranked higher. This observation cannot be justified from our existing literature review, and should be subjected to analysis using other methodologies.

Since the management style in agile approaches requires the managers to closely interact with the rest of the development team members, qualitatively monitor the progress of the project, and take quick decisions, the developers might feel getting micromanaged. This might lead to frustrations, less job satisfaction, and ultimately decrease in the productivity of the team members. These observations and the results of the data analysis ranking developer perceptions of micromanagement as an important challenge are also in conformance with the citations in the literature (Cohn and Ford, 2003; Nerur et al., 2005). Although perceptions of micromanagement are important, they can be easily overcome by educating the managers. They are not as important as the 193 
previously mentioned factors. This justifies their relative low rank. Nevertheless they are an important challenge.

Challenges such as problems with selecting the right agile methodology, developer perceptions of freedom, and team size scalability were found to be statistically insignificant. Such observations are understandable. Regarding the challenges of developer perceptions of freedom, or team size scalability - such challenges are relatively easy to mitigate by educating the managers and the developers appropriately of the potential problems surrounding the agile approaches. Also such potential perceptions are unlikely to critically affect the overall performance in team environments. So they are understandably not critical challenges, as shown by the results of data analysis. With respect to team size scalability, as mentioned in Section 7.3 , there are varying opinions regarding whether team size is an issue or not. It is not unbelievable that this varying opinion might have been reflected in the survey results as well regarding this issue. We initially hypothesized them as challenges based on reviewing the existing literature such as Cohn and Ford (2003), Nerur et al. (2005), Parrish et al. (2004), and Boehm and Turner (2003). However, none of these pieces of literature ranks these challenges, show instances, or have discussions which can be used as guides for justifying these low ranks obtained through data analysis. Therefore, we had to resort to logical justifications above in support of the observed results.

Finally, interestingly, decrease in productivity during transition ranked low and insignificant as compared to overzealousness of teams, problems with team size scalability, developer perceptions of micromanagement, or developer perceptions of 
freedom, thereby suggesting that according to the respondents there is not a significant level of decrease in productivity while transitioning from plan-driven processes to agile. Although our search of previous literature cannot substantiate this observation, intuitive reasoning suggests that since the whole approach to how software is developed changes, it is likely that there will be decrease in productivity during the transition period when developers are getting used to the new ways and approaches. 


\section{Chapter 8 \\ Conclusions}

\subsection{Summary}

Agile software development has recently gained widespread popularity. The Agile Manifesto states valuing "individuals, and interactions over processes, and tools, working software over comprehensive documentation, customer collaboration over contract negotiation, and responding to change over following a plan" (Fowler, 2002). Although there have been anecdotal and experiential reports available from practitioners of software development projects using agile practices, empirical studies on agile practices are scarce.

The overall goal of this Thesis was to advance the state-of-the-art of the research in this area. Specifically, we have addressed the following three research questions of current interest to software practitioners, in general, and (potential) agile software development practitioners, in particular:

1. What are the factors that lead to the success of software development projects, that want to adopt agile software development practices, from the perspective of agile software development practitioners?

2. What are the important changes required for adopting agile software development practices in projects practicing traditional plan-driven software development? Can we rank them according to their level of importance? 
3. What are the critical challenges/risks that projects may encounter for adopting agile software development practices in organizations practicing traditional lifecycle-based software development? Can we rank them according to their level of importance?

We undertook a survey-based ex-post-facto empirical (quantitative) study approach by identifying the success factors from the perspective of software practitioners in agile software development projects, determining the key changes traditional projects have to undergo to adopt agile practices in their projects, and the challenges/risks they have to undergo for transition. First theoretical framework was developed based on previous literature. Thereafter, different software development practitioners, practicing agile principles in their projects, were approached with both closed-ended and open-ended type questions. While the closed-ended questions formed the core of the study, the open-ended questions helped in getting fresh perspectives from currently practicing agile software development practitioners from different industry sectors. The responses were obtained from different industry sectors including those having the primary line of business as computer related (hardware/software/IS/MIS), consulting, banking/insurance, healthcare, and aerospace. The respondents belonged to both small and large sized organizations and teams. Responses came from all role types - software developers/testers, and all management levels.

The responses to the closed-ended questions were then statistically analyzed using different techniques. The important contributions are summarized in Section 7.2. 
We conducted an unprecedented large-scale empirical study. Most of the previous empirical studies have been localized within a few projects/organizations. The feedback we received while conducting the survey has been overwhelming. Many of the respondents, who are also experts in this area, have shown interest in the work and its results. Some of their comments can be found in the Chapter 5 .

In the context of the first research question it should be noted that the success factors are not the operationalized forms of the 12 principles. Some of the success factors may coincidentally map to some of the 12 principles. The hypothesized success factors are taken from what the previous pieces of literature on agile software development say are the success factors of a project. We are not testing whether the 12 principles work in practice or not. Our interest was to identify the success factors from the perspective of software practitioners in agile software development projects. For that I conducted a survey by asking different software practitioners (who had the background of following the Agile Manifesto) regarding what in their experience were the different success factors in any software development project. They did not need to be concurrent with the 12 principles. Also, someone practicing an agile software development principle may not necessarily agree that a particular principle to be a success factor.

\subsection{Contributions}

Our research contributions are as follows:

- Determining the factors that lead to the success of software development projects that want to adopt agile software development practices. We have found that 9 of the 14 hypothesized factors significantly relate with success. They are customer 
satisfaction, customer collaboration, customer commitment, decision time, corporate culture, control, personal characteristics, societal culture, and training and learning. Team distribution, team size, planning, technical competency and communication and negation were not found to have a significant relationship with success.

We also arrived at linear multiple regression models for the consolidated dependent variable success, and the regression models for five measures of success we considered in the study viz., reduced delivery schedule, increased return on investment, increased ability to meet with current customer requirements, increased flexibility to meet with changing customer requirements, and improved business processes. The regression models are summarized below:

- $\log ($ Success $)=.940+0.044($ Ind 13) $+0.031($ Ind 3) $+.020($ Ind 9) + .037(Ind 14)

- $\mathrm{D} 1=2.343+.255(\operatorname{Ind13})+.197(\operatorname{Ind14})$

- $\log (\mathrm{D} 2)=.626+.098($ Ind14) $+.048($ Ind9) $+.052($ Ind2)

- $\log (\mathrm{D} 3)=1.232+.036(\operatorname{Ind} 3)+.038(\operatorname{Ind} 7)$

- $\mathrm{D} 4=4.24+.013(\operatorname{Ind} 3)$

- $\log ($ D5) $=.722+.079(\operatorname{Ind} 4)+.052($ Ind7) $+.033(\operatorname{Ind} 3)$

The interpretations of each of the variables used in the above equations can be found in Appendix C.

- Determining the important changes required to be undergone for adopting agile software development practices. Based on the results from this study, we observe 
that the respondents opined that the Changes in Management Style are the most important (critical) changes required followed by Changes in Organization Culture, Changes in Development Processes, and Changes in Knowledge Management Strategies. Changes in Organization Culture and Changes in Development Processes have similar level of importance according to the survey results.

- Determining the important challenges/risks that should be faced for transitioning their traditional software development practices to those advocated by the agile philosophy. Based on the results from this study, we observe that the respondents opined that the three most important (critical) challenges were Upper management resistance, Resistance from developers, and Problems with geographically distributed teams (Note: this observation is purely based on the relative ordering of the mean values of the challenge items. Upper management resistance was found out to have a statistically significantly greater mean value than the Resistance from developers, whereas the mean value of Resistance from developers was not found to have a statistically different mean of Problems with geographically distributed teams).

- Determining additional success factors, changes, and challenges, if any. From the responses obtained from the open-ended questions, we were successful in finding additional success factor, and changes. No significant challenge categories were identified. Among others four seemingly important success factor dimensions have emerged. They are: Learning from failure, Timing issues, Other team 
characteristics, and Use of tools. Similarly, two seemingly important change categories emerged. They are: Changes in customer attitude and Changes in personal characteristics. The above items did not initially appear in our hypothesized list of success factors, and changes. This is because we hypothesized all the success factors, and changes based on our search of existing literature, and our search of existing literature did not find sufficient evidences supporting the consideration of such factors in the literature. This, however, does not indicate that these newly suggested success factors or changes are not important. As the identification of such success factors and changes is a complex problem guided by several factors, we call for additional research to examine them.

\subsection{Future Work}

Many issues are worthy of detailed investigation in the future. Some of them are outlined below:

1. Further research should be done to validate the results obtained from this study in practice. Practitioners should attempt to use the results obtained in this study in actual projects and test the degree of usefulness of the results in practice. This is important because although the results obtained in this Thesis have been obtained by statistically analyzing the responses from currently practicing agile software professionals, unless they are deemed to be of use in practice, the research results would be of limited use other than serving as an inspiration for future researchers to conduct further research in this direction. 
2. Due to the nature of these studies, controlled experiments, where by one factor is kept constant while varying others to different degrees, are difficult to conduct in real-life projects. To overcome that it is suggested that computer-based simulation studies should be performed to validate the results obtained from this study. Such controlled experiments would be helpful to examine the detailed behavior/characteristics of one particular issue.

3. In this Thesis, additional success factors, changes and challenges were suggested by several respondents as a part of open-ended questions. Future work should target empirically evaluate them.

4. In this Thesis, we have analyzed the consolidated data obtained from various respondents of different continents around the world. It would be interesting to conduct a detailed investigation of the transcontinental/cultural differences in the success factors, critical changes, and critical challenges, because as we have seen to some extent in this Thesis, agile software development is influenced by behavioral/personal characteristics of respondents. Such an investigation would require capturing not only the country/continent of origin of the respondent, but also their places of work. There is a significant amount of complexity involved in this kind of research, because in today's global economy, organizations are distributed throughout the world, many employees travel across continents, they might have been educated/trained in one culture, but their work behavior has changed over the years by working in different cultural environments. The 
investigation of this issue was scoped out of the Thesis. However, it is worth investigating in the future.

5. Similar to the point mentioned above, whether the project size, project complexity, or the organization size matters or not was scoped out from investigation as part of this Thesis. While the value of such a work was appreciable, it was felt that inclusion of such a research question would increase the length of the already long questionnaire (it should be noted that many people have commented critically about the size of the questionnaire). However, in this Thesis, I have collected such information to get a statistic of the size/complexity of the organizations/projects of the respondents of the survey. This was done with the idea that these statistics would hopefully help us to get an insight about the type of respondents of the survey whose responses have produced the results that we have obtained. Whether project size, organization size, or project complexity matters or not is a different question altogether from the ones that we investigated in this Thesis. They are worth investigating in the future, but to draw firm, and meaningful conclusions, one needs to do an in-depth study (a controlled simulation study is suggested) by varying the above parameters, and framing all the research questions accordingly.

6. In this Thesis, we have analyzed consolidated data obtained from all kinds of sectors. It would be very interesting to investigate if there are any differences in success factors, critical changes and critical challenges across different sectors. Detailed studies, perhaps more appropriately detailed case studies, are required to 
be performed to draw any meaningful conclusions regarding this issue. We encourage further work on this issue in the future.

7. We have analyzed data by assuming the data obtained across different work functions are equally important. It would be quite interesting to investigate if at all there will be any differences in the results based on the work functions of the respondents.

8. The role of experience/competency in agile software development has been debated in the literature. We have analyzed data assuming all levels of experience equally important. It would be required to investigate if there are any differences in the results based on the levels of experience. Detailed case studies are required to draw any meaningful conclusions regarding such an issue.

9. It is important to investigate if there are any differences in the results obtained at different levels of agility, i.e., organizations which are highly agile versus the ones that are moderately agile or not much agile at all.

10. In this Thesis, we did not consider technical factors. We focused on management, organizational, and people centric factors. The analysis of technical factors is a substantial, important area that requires future attention in the future. This requires the knowledge about software development, and can be best done in conjunction with developers working on practical real-life projects.

11. The influence of type and complexity of projects should also be investigated. Complexity has many dimensions such as technical and economic dimensions. 
12. It is necessary for conducting large-scale interviews. From our study we realized that practitioners have a lot to say. Several new dimensions might pop up from interviews, which were not evident as such through closed-ended survey questionnaire administration.

13. In this Thesis we studied agile practices in general. The results obtained do not provide an in-depth understanding of how they relate to the specific agile software development methodologies such as Scrum, XP, and DSDM. It would be interesting to study the success factors, changes, and challenges that particularly concern the individual agile software development methodologies that are guided by the agile principles mentioned in Chapter 2 .

14. The nature of the research methodology we considered constrained us from gaining useful environmental, and in-depth understanding of issue, which might be possible through site visits of the individual respondents. Although such an approach restricts the scalability of the study, they are helpful in gaining in-depth understanding of issues. Since our research results in this Thesis provides an exploratory level understanding of the success factors, changes, and challenges, in-depth understanding of issues is called for. 


\section{References}

P. Abrahamson, O. Sallo and J. Ronkeinen. 2002. Agile software development methods $\begin{array}{lllll}- & \text { review } & \text { and } & \text { analysis. } & V T T\end{array}$ http://www.inf.vtt.fi/pdf/publications/2002/P478.pdf (Last accessed: December 2005).

P. Abrahamson and J. Koskela. 2004. Extreme Programming: A Survey of Empirical Data from a Controlled Case Study. Proceedings of the 2004 International Symposium on Empirical Software Engineering (ISESE'04). Redondo Beach, California, USA. pp. 7392.

W.R. Adrion. 1993. Research Methodology in Software Engineering. Summary of the Dagstuhl Workshop on Future Directions in Software Engineering. ACM SIGSOFT Software Engineering Notes. Vol. 18. No. 1. pp. 36-37.

S.W. Ambler. 2002a. Lessons in Agility from Internet-Based Development. IEEE Software. March/April. pp. 66-73.

S.W. Ambler. 2002b. Agile Modeling. John Wiley \& Sons.

S.W. Ambler. 2005a. Where is the Proof that Agile Methods Work. Online article that can be found at http://www.agilemodeling.com/essays/proof.htm (Last accessed: December 2005).

S.W. Ambler. 2005b. Communication on Agile Software Projects. Online article that can be found at http://www.agilemodeling.com/essays/communication.htm (Last accessed: December 2005). 
S.W. Ambler. 2005c. Remaining Agile. Online article that can be found at http://www.agilemodeling.com/essays/remainingAgile.htm (Last accessed: December 2005).

S. Ambler. 2005d. Quality in an Agile World. Software Quality Professional. Vol. 7. No. 4. pp. 34-40.

D. Anderson. 2002. Morning Roll Call. http://dn.codegear.com/article/29686 (Last accessed: March 4, 2007).

D. J. Anderson. 2003. Agile Management for Software Engineering: Applying the Theory of Constraints for Business Results. Prentice Hall PTR, ISBN 0-13-142460-2.

P. Baheti, L. Williams, E. Gehringer, D. Stotts and J.M. Smith. 2002. Distributed Pair Programming: Empirical Studies and Supportive Environments. Technical Report TR02010. Department of Computer Science. University of North Carolina at Chapel Hill. March.

R. Balzer, T.E. Cheetham and C. Green. 1983. Software Technology in the 1990s: Using a New Paradigm, Computer, November, pp. 39-45.

H. D. Bengington. 1956. Production of Large Computer Programs. Proceedings of the ONR Symposium on Advanced Programming Methods for Digital Computers, June, pp. $15-27$.

B. Boehm. 1981. Software Engineering Economics. Prentice Hall.

B. W. Boehm. 1988. A Spiral Model of Software Development and Enhancement. Computer, May, pp. 61-72. 
B. Boehm and R. Turner. 2003. Observations on Balancing Discipline and Agility. Proceedings of the Agile Development Conference (ADC'03). June. Salt Lake City, Utah, USA.

B. Boehm and R. Turner. 2005. Management Challenges to Implementing Agile Processes in Traditional Development Organizations. IEEE Software. September/October 2005, pp. 30-39.

L. Bossavit. 2002. The Unbearable Lightness of Programming: A Tale of Two Cultures. Cutter IT Journal. Vol. 15, No. 9, pp. 5-11.

T.A. Boyle. 1999. Quality Management in the R\&D Departments of Quality Award Winning Manufacturing Organizations. MMS Thesis. Eric Sprott School of Business. Carleton University. Ottawa, Ontario, Canada.

T.A. Boyle. 2003. Organizational Diffusion of Integrated Product Developmebt: Determinants and Performance Consequences. PhD Thesis. Eric Sprott School of Business. Carleton University. Ottawa, Ontario, Canada.

A. Cherns. 1976. The Principles of Sociotechnical Design. Human Relations. Vol.2. No. 9. pp 783-792.

C. W. Clegg. 2000. Sociotechnical Principles for Systems Design. Applied Ergonomics. Vol. 31. pp 463-477.

V.T. Clover and H.L. Balsley. 1979. Business Research Methods. $2^{\text {nd }}$ Edn. Grid Publishing, Inc. Columbus, Ohio.

A. Cockburn. 2001. Agile Software Development. Addison-Wesley, $1^{\text {st }}$ Edition. 208 
A. Cockburn and J. Highsmith. 2001. Agile Software Development: The People Factor. Software Management. November. pp. 131-133.

A. Cockburn. 2002a. Learning From Agile Software Development - Part One.

CrossTalk: Journal of Defense Software Engineering. October.

http://www.stsc.hill.af.mil/crosstalk/2002/10/cockburn.html

A. Cockburn. 2002b. Learning From Agile Software Development - Part Two. CrossTalk: Journal of Defense Software Engineering. November. pp. 9-12.

M. Cohn and D. Ford. 2003. Introducing an Agile Process to an Organization. IEEE Computer. June. pp. 74-78.

L. Copeland. 2001. Developers Approach Extreme Programming with Caution. Computerworld. October 22.

http://www.computerworld.com/industrytopics/automotive/story/0,10801,64957,00.html (Last accessed: March 4, 2007).

J.W. Creswell. 2003. Research Design: Qualitative, Quantitative, and Mixed Methods Approaches. Sage Publications. USA.

L. Crispin. 2005. User Customer Tests to Drive Development. http://www.methodsandtools.com/archive/archive.php?id=23 (Last accessed: March 5, 2007).

R. Deias, G. Mugheddo and O. Murro. 2002. Introducing Agile in a Startup. http://www.agilealliance.org/system/article/file/873/file.pdf (Last accessed: March 4, 2007). 
W.H. Delone and E.R. McLean. 1992. Information Systems Success: The Quest for the Dependent Variable. Information Systems Research, Vol. 3, No. 1, pp. 60-95.

W.H. Delone and E.R. McLean. 2003. The DeLone and McLean Model of Information Systems Success: A Ten Year Update. Journal of Management Information Systems. Vol. 19, No. 4, pp. 9-30.

A. v. Deursen. 2001. Customer Involvement in Extreme Programming. http://homepages.cwi.nl/ arie/wci2001/wci-report.pdf (Last accessed: March 5, 2007)

T. Dingsoyr and G.K. Hansen. 2004. Extending Agile Methods: Postmortem Reviews as Extended Feedback. Lecture Notes in Computer Science. Vol. 2630. pp. 4-12.

J. Drobka, D. Noftz and R. Raghu. 2004. Piloting XP on Four Mission-Critical Projects. IEEE Software. November/December. pp70-75.

T. Dyba. 2000. Improvisation In Small Software Organizations. IEEE Software. Vol. 17. No. 5. pp. $82-87$.

K. El Emam. 2004. The ROI from Software Quality: An Executive Briefing. $3^{\text {rd }}$ Edn., Ottawa Software Quality Association. ISBN 0-9734744-0-8.

A. Elssamadisy. 2001. XP on a Large Project - A Developer's View. http://www.xpuniverse.com/2001/pdfs/EP202.pdf (Last accessed: December 2005).

eWorkshop. 2002. Summary of the First eWorkshop on Agile Methods. http://fcmd.umd.edu/projects/Agile/Summary/SummaryPF.htm. April. 
C. Farrell, R. Narang, S. Kapitan and H. Webber. 2002. Towards an Effective Onsite Customer Practice. http://www.agilealliance.org/system/article/file/1014/file.pdf (Last accessed: March 5, 2007).

M. Fowler and J. Highsmith. 2001. The Agile Manifesto. Software Development Magazine. August. http://www.sdmagazine.com/documents/s=844/sdm0108a/0108a.htm (Last accessed: December 2005).

M. Fowler. 2002. The Agile Manifesto: Where It Came From and Where It May Go. http://martinfowler.com/articles/agileStory.html (Last accessed: December 2005).

M. Fowler. 2006. Using an Agile Software Development Process with Offshore Development. http://www.martinfowler.com/articles/agileOffshore.html (Last accessed: March 4, 2007).

J. Gawlas. 2003. Mission-Critical Development with XP \& Agile Processes. Dr. Dobb's Journal. December Issue. http://www.ddj.com/184405520 (Last accessed: March 4, 2007).

L. Geeck. 2005. Offshore Improvements. Software Development Magazine. May. http://www.sdmagazine.com/documents/s=9693/sdm0505g/sdm0505g.html (Last accessed: December 2005).

R. Gittins, S. Hope and I. Williams. 2001. Qualitative Studies of XP in a Medium Sized Business. Proceedings of the $2^{\text {nd }}$ International Conference on Extreme Programming and Flexible Processes in Software Engineering. Sardina, Italy. May.

E. M. Goldratt. 1990. What is this thing called Theory of Constraints and how should it be implemented? North River Press, Great Barrington, MA, USA. ISBN 0-88427-166-8. 
E. M. Goldratt. 1998. Essays on the Theory of Constraints. North River Press, Great Barrington, MA, USA. ISBN 0-88427-159-5.

A. Gopal, M.S. Krishnan, T. Mukhopadhyay and D.R. Goldenson. 2002. Measurement Programs in Software Development: Determinants of Success. IEEE Transactions on Software Engineering. Vol. 28. No. 9. pp. 863-875

L.A. Graffin. 2001. A Customer Experience: Implementing XP. http://www.agilealliance.org/system/article/file/930/file.pdf (Last accessed: March 4, 2007).

N.B. Harrison. 2003. A Study of Extreme Programming in a Large Company. http://www.agilealliance.org/system/article/file/1292/file.pdf (Last accessed: March 4, 2007).

J. Highsmith. 2000. Retiring Lifecycle Dinosaurs. Software Testing and Quality Engineering Magazine. http://www.jimhighsmith.com/articles/Dinosaurs.pdf (Last accessed: December 2005).

J. Highsmith. 2004. Agile Project Management. Addison Wesley.

A. Irons. 2006. Agile Methods Silver Bullet or Red Herring. Northumbria University. UK. An online document that can be found at http://www.newcastle.bcs.org/BCS\%20Agile\%20methods.ppt (Last accessed: July 2006) [The year 2006 mentioned in this reference corresponds to the year of last access of this online document. The year of publication of this document is unknown]. 
O. Jensen. 2002. Time Constrained Requirements Engineering - The Cooperative Way. http://www.agilealliance.org/system/article/file/913/file.pdf (Last accessed: March 4, 2007).

J. Kalermo and J. Rissanen. 2002. Agile Software Development in Theory and Practice. Master's Thesis, Department of Computer Science and Information Systems. University of Jyvaskyla. Finland.

E. Karahanna, D.W. Straub, N. L. Chervany. 1999. Information Technology Adoption Across Time: A Cross-Sectional Comparison of Pre-Adoption and Post-Adoption Beliefs. MIS Quarterly. Vol. 23. No. 2. pp. 183-213.

E. R. Keith. 2002. Agile Software Development Processes: A Different Approach to Software Design. http://www.agilealliance.com/articles/keitheveretteragileso/file (Last accessed: December, 2005).

N. Kini and S. Collins. 2003. Steering the Car: Lessons Learned from an Outsourced XP Project. http://www.xpuniverse.com/2001/pdfs/XPU03.pdf (Last accessed: December 2005).

J. Kontio. Software Engineering Risk Management: A Method, Improvement Framework, and Empirical Evaluation. Doctoral Dissertation. Department of Computer Science and Engineering, Helsinki University of Technology, Finland.

U. Kumar, M. Maheshwari and V. Kumar. 2004. A Framework for Achieving Ebusiness Success. Journal of Industry and Higher Education. Vol. 18, No. 1, pp. 47-51. 
C. Larman. 2004. Agile \& Iterative Development: A Manager's Guide. AddisonWesley.

M. Lazaro and E. Marcos. 2005. Research in Software Engineering: Paradigms and Methods. Proceedings of the First International Workshop on Philosophical Foundations of Information Systems Engineering. Porto, Portugal. June.

M. Lindvall, V. Basili, B. Boehm, P. Costa, K. Dangle, F. Shull, R. Tesoriero, L. Williams and M. Zelkowitz. 2002. Empirical Findings in Agile Methods. Proceedings of Extreme Programming and Agile Methods - XP/Agile Universe 2002, pp. 197-207.

A. Mahanti. 2006. Challenges in Enterprise Adoption of Agile Methods - A Survey. Journal of Computing and Information Technology, Vol. 14, No. 3, pp. pp. 197-206.

B. Maheshwari, V. Kumar and U. Kumar. 2006. Optimizing Success in Supply Chain Partnerships. Journal of Enterprise Information Management, Vol. 19, No. 3, pp. 277291.

M. Maheshwari. 2002. Critical Success Factors of E-Business: An Empirical Study. M.B.A. Thesis. Eric Sprott School of Business. Carleton University, Ottawa, Ontario, Canada.

D. D. McCracken and M. A. Jackson. 1982. Life-Cycle Concept Considered Harmful. ACM Software Engineering Notes, April, pp. 29-32.

P.E. McMahon. 2004. Bridging Agile and Traditional Development Methods: A Project Management Perspective. CrossTalk: The Journal of Defense Software 
Engineering. May. http://www.stsc.hill.af.mil/crosstalk/2004/05/0405McMahon.html (Last accessed: December 2005).

S. Nerur, R. Mahapatra and G. Mangalaraj. 2005. Challenges of Migrating to Agile Methodologies. Communications of the ACM. Vol. 48, No. 5, May, pp. 73-78.

Net Worth Consulting. 2004. Time/Cost/Quality Trade-offs in the Budget Process. Quantum ${ }^{2}$ White Paper 10.25.04. Leadership Series: Measurement. http://quantum.dialog.com/q2_resources/whitepapers/budget_process.pdf (Last accessed: December 2005).

W.L. Newman. 2005. Social Research Methods: Quantitative and Qualitative Approaches. Allyn \& Bacon.

Nucleus Research. 2002. Measuring Return on Investment Quick Reference Guide. Research Note B20. http://www.nucleusresearch.com/research/b20.pdf (Last accessed: August 11, 2006).

D. Opperthauser. 2003. Defect Management in an Agile Development Environment. CrossTalk: The Journal of Defense Software Engineering. September. http://www.stsc.hill.af.mil/crosstalk/2003/09/0309Opperthauser.html (Last accessed: December 2005).

OST. 1. Open Systems Theory. http://en.wikibooks.org/wiki/Systems_Theory/Open/Closed_System_Structure OST. 2. Open Systems Theory. http://faculty.ncwc.edu/TOConnor/417/417lect01.htm 
F. Paetsch, A. Eberlin and F. Maurer. 2003. Requirements Engineering and Agile Software Development. Proceedings of the $12^{\text {th }}$ International Workshops on Enabling Technologies: Infrastructure for Collaborative Enterprises (WETICE'03). Austria.

A. Parrish, R. Smith, D. Hale and J. Hale. 2004. A Field Study of Developer Pairs: Productivity Impacts and Implications. IEEE Software. Vol. 21. No. 5. pp. 76-79.

S.M. Pine. 2001. An Application of XP in a Multiple Team/Multi-Process Environment. http://www.agilealliance.com/articles/pinestephenmanapplica/file (Last accessed: December 2005).

A.N.S. Persaud. 1999. Synergistic Innovations in Internationally Dispersed $R \& D$ Labs. PhD Thesis. Eric Sprott School of Business. Carleton University. Ottawa, Ontario, Canada.

S.L. Pfleeger. 1995. Experimental Design and Analysis in Software Engineering. Annals of Software Engineering. Vol. 1. pp. 219-253.

S.M. Pine. 2001. Application of XP in a Multiple Team/Multi Process Environment. http://www.agilealliance.org/system/article/file/1000/file.pdf (Last accessed: March 4, 2007).

M. Poppendieck and T. Poppendieck. 2003. Lean Software Development: An Agile Toolkit. Addison-Wesley.

D.J. Power, A.S. Sohal and S.U. Rahman. 2001. Critical Success Factors in Agile Supply Chain Management: An Empirical Study. International Journal of Physical Distribution \& Logistics Management. Vol. 31. No. 4. pp. 247-265. 
PMI. 2004. Project Management Body of Knowledge (PMBOK). $3^{\text {rd }}$ Edition. Project Management Institute. USA.

J. Rasmusson. 2003. Introducing XP into Greenfield Projects: Lessons Learned. IEEE Software, March/April Issue.

http://www.computer.org/portal/site/software//homepage/2003/s3ras.htm (Last accessed: March 4, 2007).

D.J. Reifer. 2002. How Good Are Agile Methods? IEEE Software. July/August. pp. 1417.

W. W. Royce. 1987. Managing the Development of Large Software Systems: Concepts and Techniques, Proceedings of Wescon, August. (Also available in Proceedings of ICSE9, IEEE Computer Society Press, 1987).

B. Rumpe and A. Schroder. 2002. Quantitative Survey on Extreme Programming Projects. Proceedings of International Conference on Extreme Programming and Flexible Processes in Software Engineering (XP2002). Alghero. Italy. May. pp. 95-100.

B. Schatz, K. Schwaber and R.C. Martin. 2004. Best Practices in Scrum Project Management and XP Agile Software Development. White Paper. Object Mentor, Inc. http://www.objectmentor.com/resources/articles/Primavera. July.

K. Schwaber and M. Beedle. 2002. Agile Software Development with Scrum. Prentice Hall, New Jersey, USA.

K. Schwaber. 2004. Agile Project Management with Scrum. Microsoft Press, Redmond, Washington, USA. 
Shine Technologies. 2003. Agile Methodologies: Survey Results. Shine Technologies Pty Ltd. http://www.shinetech.com (Last accessed: December 2005).

G. Sleve and R. Gioia. Agile Before Agile Was Cool. 2002. Agile Before Agile Was Cool. CrossTalk: The Journal of Defense Software Engineering. October. http://www.stsc.hill.af.mil/crosstalk/2002/10/sleve.html (Last accessed: December 2005).

P.G. Smith and R. Pichler. 2005. Agile Risks, Agile Rewards. Software Development Magazine. April. pp. 50-53.

M.R. Spiegel and L.J. Stephens. 1998. Theory and Problems of Statistics. $3^{\text {rd }}$ Edn. McGraw Hill, Schaum's Outline Series.

SST. 1. Sociotechnical Systems Theory. http://en.wikipedia.org/wiki/Sociotechnical_systems_theory

M. Stephens and D. Rosenberg. 2003. Extreme Programming Refactored: The Case Against XP. Apress L.P., Berkeley, California.

J. Stevens. 1996. Applied Multivariate Statistics for the Social Sciences. Lawrence Erblaum Associates, Hillsdale, NJ.

L. Thorup and O. Jepsen. 2003. Report on XP Workshop at JAOO 2003 - Improving Customer Developer Collaboration.

http://www.agilealliance.org/article/articles_by_category/19

TOC. 1. Theory of Constraints and Agile Software Development. http://theagileblog.net/2006/03/theory_of_constraints_and_agil_1.html

TOC. 2. Theory of Constraints. http://en.wikipedia.org/wiki/Theory_of_constraints 
J.-P. Tolvanen. 1998. Incremental Method Engineering With Modeling Tools: Theoretical Principles and Empirical Evidence. Doctoral Dissertation. University of Jyvaskyla, Jyvaskyla.

D. Turk, R. France and B. Rumpe. 2002. Limitations of Agile Software Processes. In the Proceedings of the $3^{\text {rd }}$ International Conference on Extreme Programming and Agile Processes in Software Engineering. Sardinia. Italy.

R. Turner and B. Boehm. 2003. People Factors in Software Management: Lessons From Comparing Agile and Plan Driven Methods. CrossTalk: The Journal of Defense Software Engineering. December. pp. 4-8.

L. Williams and R. Kessler. 2002. Overcoming Management Resistance to Pair Programming. Chapter 4 in Pair Programming Illuminated. Addison Wesley Professional. $1^{\text {st }}$ Edition.

M.V. Zelkowitz and D.R. Wallace. 1998. Experimental Models for Validating Technology. IEEE Computer. Vol. 31. No. 5. pp. 23-31. 


\section{Appendix A \\ Covering Letters}

\section{A.1. Letter I \\ Main letter accompanying the survey questionnaires sent by post}

Dear Sir/Madam:

Let me at first introduce myself. My name is Subhas C. Misra. I am a PhD degree candidate in the Eric Sprott School of Business, Carleton University, Ottawa, Ontario, Canada. As part of my Thesis, I am conducting a survey on Agile Software Development (ASD) practices. Specifically, I am trying to assess the success factors of projects from the perspective of ASD practitioners, the important changes required and the challenges involved in adopting these practices.

I would be extremely grateful if you would kindly help me in my research pursuits by completing the attached questionnaire. You have been particularly selected to fill out this questionnaire because we felt that you are knowledgeable about and/or experienced working with some or all of the ASD principles. Please note that the questionnaire is designed in such a way that you might be able to fill it out, even if that is strictly not the case. This questionnaire may be completed by any software practitioner, e.g., director, senior manager, manager, architect, team leader, developer, tester, who has experience practicing software development using both plan-driven (traditional) and agile approaches and methodologies.

All your responses will be kept strictly confidential. The results of the study will be aggregated and/or reported in such a way that no single person, project team, or organization is identified. All data collected from this survey would be stored in a password protected personal computer and would be destroyed on completion of this research project. Thus, we do not see any risk to you or your company if you complete this questionnaire. Once again, your response will significantly help me in completing my PhD degree successfully in time. In addition, by responding to the questionnaire you will be joining me in attempting to benefit the global community of software practitioners by providing an improved understanding of ASD practices. You can also benefit from receiving a complimentary copy of the research report upon request.

Should you be interested in further information about this study, please feel free to contact me at scmisra@connect.carleton.ca or my supervisor, Professor Vinod Kumar, at vinod kumar@carleton.ca (Telephone: 613-520-2379). Please also feel free to let me know if you are interested in knowing the final results of the study.

Finally, thank you very much in taking your valuable time considering responding to the enclosed questionnaire. Your input is very much appreciated.

Sincerely yours,

Subhas C. Misra, Ph.D. Student

Eric Sprott School of Business

Carleton University

1125 Colonel By Drive

Ottawa, Ontario, K1S 5B6, CANADA

E-mail: scmisra@connect.carleton.ca 


\section{A.2. Letter II}

Forwarding letter sent to the human resource departments of the Agile Corporate Member Organizations

Dear Sir/Madam,

Let me at first introduce myself. My name is Subhas C. Misra. I am a PhD student in the Eric Sprott School of Business, Carleton University, Ottawa, Ontario, Canada. As part of my Thesis, I am conducting a survey on Agile Software Development (ASD) practices. Specifically, I am trying to assess the factors that will influence the success of projects that want to adopt ASD practices, the important changes required, and the challenges involved in adopting these practices.

I am contacting you because your organization is listed as a corporate member of the Agile Alliance (http://www.agilealliance.org/agiledevelopment/portal_url/corporatemembers). There are 5 survey questionnaire booklets enclosed along with this letter. Could you kindly forward each of the 5 attached questionnaires to 5 different software practitioners (preferably belonging to different teams) in your organization?

The questionnaires may be completed by any software practitioner, e.g., director, senior manager, manager, architect, team leader, developer, tester, who has experience practicing software development using both plan-driven (traditional) and agile approaches and methodologies.

All the responses from the survey will be kept strictly confidential. The results of the study will be aggregated and/or reported in such a way that no single person, project team, or organization is identified. All data collected from this survey would be stored in a password protected personal computer and would be destroyed on completion of this research project. Thus, we do not see any risk to you or your company if you forward this questionnaire.

Once again, your help in forwarding this survey questionnaire to the above mentioned software development teams will significantly help me in completing my $\mathrm{PhD}$ degree successfully in time. In addition, by responding to the questionnaire your organization's software development teams will be joining me in attempting to benefit the global community of software practitioners an improved understanding of ASD practices. The survey respondents may also benefit from receiving a complimentary copy of the research report upon request.

Should you be interested in further information about this study, please feel free to contact me at scmisra@connect.carleton.ca or my supervisor, Professor Vinod Kumar, at vinod_kumar@carleton.ca. Please also feel free to let me know if you are interested in knowing the final results of the study.

Finally, thank you very much in taking your valuable time considering helping me in my research pursuits.

Sincerely,

Subhas C. Misra 


\section{A.3. Letter III}

\section{Letter sent by e-mail to the contact persons of Agile User Groups worldwide}

Dear xxx:

Let me at first introduce myself. My name is Subhas C. Misra. I am a PhD degree candidate in the Eric Sprott School of Business, Carleton University, Ottawa, Ontario, Canada. As part of my Thesis, I am conducting a survey on Agile Software Development (ASD) practices. Specifically, I am trying to assess how ASD practices affect the performance of software development projects, the important changes required and the challenges involved in adopting these practices.

I am contacting you because you are the contact person for the agile user group xxxxx. I would be extremely grateful if you would kindly help me in my $\mathrm{PhD}$ research pursuits by completing the survey questionnaire that can be obtained by clicking on the link below:

http://www.surveymonkey.com/s.asp?u=488032873168

In addition, I would request you to kindly forward the above survey questionnaire link to the other members of your user group, and the organization in which you work, and request/encourage them to complete the survey as well.

By responding to the questionnaire you and the members of your user group will be joining me in attempting to benefit the global community of software practitioners by providing an improved understanding of ASD practices. You can also benefit from receiving a complimentary copy of the research report upon request.

This questionnaire may be completed by any software practitioner, e.g., director, senior manager, manager, architect, team leader, developer, tester, who has experience practicing software development using both plan-driven (traditional) and agile approaches and methodologies.

Please note that all your responses will be kept strictly confidential. The results of the study will be aggregated and/or reported in such a way that no single person, project team, or organization is identified. All data collected from this survey would be stored in a password protected personal computer and would be destroyed on completion of this research project. Thus, we do not see any risk to you or your company if you complete this questionnaire.

Once again, your response will significantly help me in completing my $\mathrm{PhD}$ degree successfully in time. Should you be interested in further information about this study, please feel free to contact me at scmisra@connect.carleton.ca or my supervisor, Professor Vinod Kumar, at vinod kumar@carleton.ca (Telephone: 613-520-2379). Please also feel free to let me know if you are interested in knowing the final results of the study.

Kindly respond to this message letting me know about your participation. Finally, thank you very much in taking your valuable time considering responding to the enclosed questionnaire. Your input is very much appreciated.

Sincerely yours,

Subhas C. Misra 


\section{Appendix B \\ Survey Questionnaire}

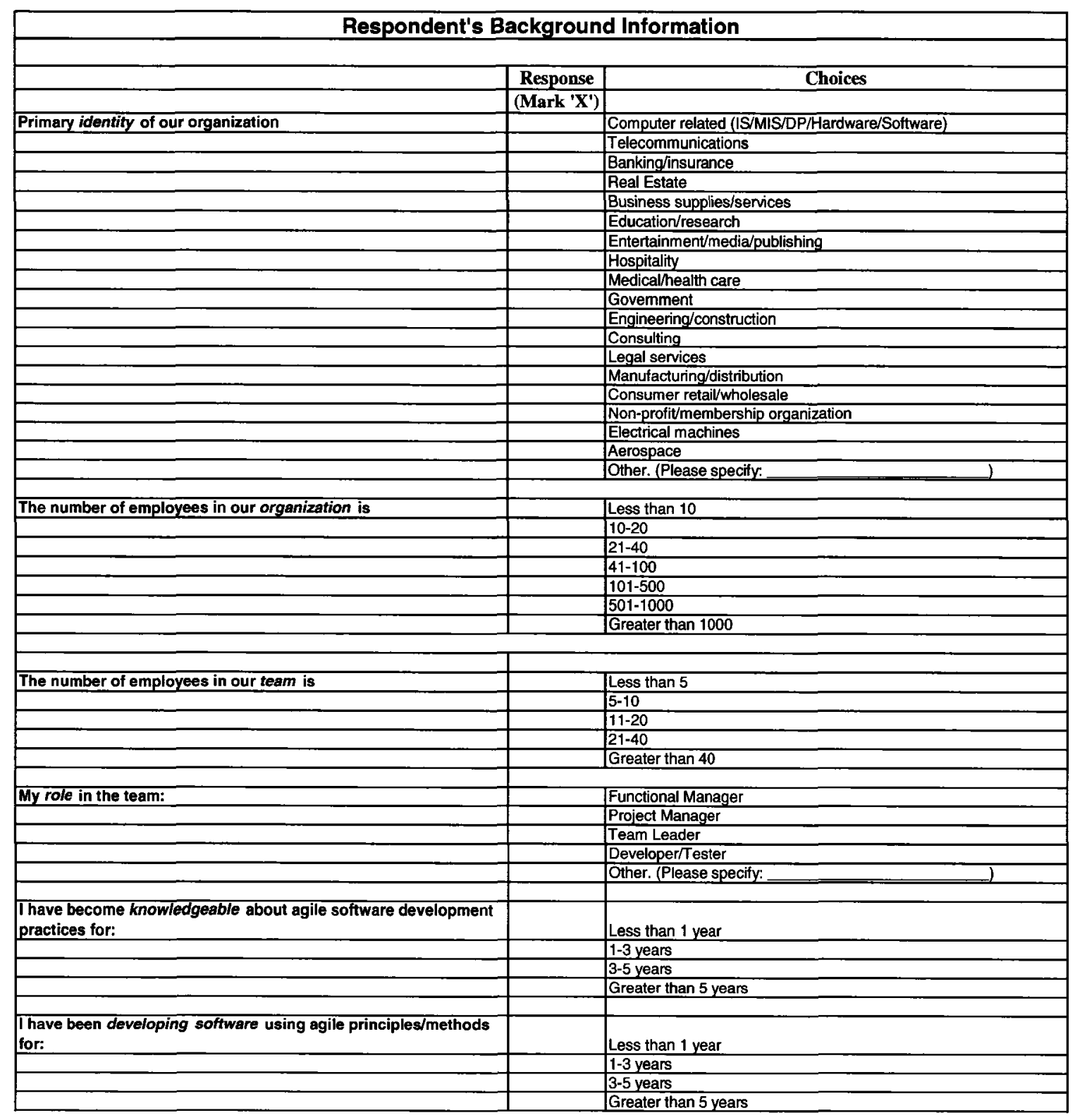


The checklist below will help in assessing the degree of practice of agile software development principles by your team. It contains 12 statements. For each of the principles, please mark in the scale of 1 (disagree) 105 (strongly agree) your degree of practice of each of the principles.

Checklist

\begin{tabular}{|c|c|c|c|c|c|c|}
\hline \multirow[b]{2}{*}{ We practice this principle } & \multicolumn{6}{|c|}{ Response } \\
\hline & $\begin{array}{l}\text { Strongly } \\
\text { Disagree }\end{array}$ & $\begin{array}{c}\text { Somewhat } \\
\text { Disagree }\end{array}$ & $\begin{array}{c}\text { Neither } \\
\text { Disagree } \\
\text { nor Agree }\end{array}$ & $\begin{array}{c}\text { Somewhat } \\
\text { Agree }\end{array}$ & $\begin{array}{c}\text { Strongly } \\
\text { Agree }\end{array}$ & $\begin{array}{c}\text { Not } \\
\text { Applicable } \\
\text { or Don't } \\
\text { know }(X)\end{array}$ \\
\hline & 1 & 2 & 3 & 4 & 5 & \\
\hline $\begin{array}{l}\text { We give high priority to satisfying customers through early and } \\
\text { continuous delivery of valuable software. }\end{array}$ & & & & & & $\mathbf{x}$ \\
\hline We welcome changing requirements, even late during development. & & & & & & $\bar{x}$ \\
\hline $\begin{array}{l}\text { We deliver working software more frequently, from couple of weeks to a } \\
\text { couple of months, with a preference to a shorter timescale }\end{array}$ & & & & & & $\mathbf{x}$ \\
\hline $\begin{array}{l}\text { Our business people and developers work together daily (very closely) } \\
\text { throughout the project }\end{array}$ & & & & & & $\mathbf{x}$ \\
\hline $\begin{array}{l}\text { We build projects around motivated individuals. We give them the } \\
\text { environment and support they need, and trust them to get the job done. }\end{array}$ & & & & & & $\mathbf{x}$ \\
\hline $\begin{array}{l}\text { We emphsize more on face-to-face communication for conveying } \\
\text { information to and within the development team. }\end{array}$ & & & & & & $\mathbf{x}$ \\
\hline We measure and track progress based on working software. & & & & & & $\bar{x}$ \\
\hline $\begin{array}{l}\text { We promote sustainable development. Our sponsors, developers, and } \\
\text { users maintain a constant pace indefinitely. }\end{array}$ & & & & & & $x$ \\
\hline $\begin{array}{l}\text { Our software development project team follows continuous attention to } \\
\text { technical excellence and good design for development. }\end{array}$ & & & & & & $\mathbf{x}$ \\
\hline $\begin{array}{l}\text { We practice simple designs, processes, and approaches in our } \\
\text { software development methodologies. We implement features that are } \\
\text { required by the customers -- nothing more. }\end{array}$ & & & & & & $\mathbf{x}$ \\
\hline $\begin{array}{l}\text { Our development teams are self-organizing - our teams can (re)- } \\
\text { organize continuously in different configurations to meet the changing } \\
\text { requirements and the newly arising challenges of the business. }\end{array}$ & & & & & & $\mathbf{x}$ \\
\hline $\begin{array}{l}\text { At regular intervals, our team reflects on how to become more effective, } \\
\text { then tunes and adjusts its behaviour accordingly. }\end{array}$ & & & & & & $\mathbf{x}$ \\
\hline
\end{tabular}

\section{Success Factors Survey}

Please indicate your level of agreement of the following statements based on your experience of agile software development practices in your team within your organization. Please mark your choice on a scale of 1 to 5 , where 1 is "Strongly Disagree", and 5 is "Strongly Agree". Your replies should reflect your best guess from your knowledge and experience of software development projects performed by your team in your organization.

\begin{tabular}{|l|c|c|c|c|c|c|}
\hline & $\begin{array}{c}\text { Strongly } \\
\text { Disagree }\end{array}$ & $\begin{array}{c}\text { Somewhat } \\
\text { Disagree }\end{array}$ & $\begin{array}{c}\text { Neither } \\
\text { Disagree } \\
\text { nor Agree }\end{array}$ & $\begin{array}{c}\text { Somewhat } \\
\text { Agree }\end{array}$ & $\begin{array}{c}\text { Strongly } \\
\text { Agree }\end{array}$ & $\begin{array}{c}\text { Not } \\
\text { Applicable } \\
\text { or Don't }\end{array}$ \\
\hline Customer Satisfaction & & & & & & \\
\hline $\begin{array}{l}\text { In our projects, we give very high priority to achieving } \\
\text { customer satisfaction. }\end{array}$ & 1 & 2 & 3 & 4 & 5 & $\mathrm{X}$ \\
\hline
\end{tabular}

\begin{tabular}{|l|c|c|c|c|c|c|}
\hline Customer Collaboration & & & & & & \\
\hline $\begin{array}{l}\text { In our projects, customers closely collaborate with the } \\
\text { development team members. }\end{array}$ & 1 & 2 & 3 & 4 & 5 & $x$ \\
\hline
\end{tabular}

\begin{tabular}{|l|l|l|l|l|l|l|}
\hline Customer Commitment & & & & & & \\
\hline $\begin{array}{l}\text { In our software development projects, customers are } \\
\text { commited to the project, i.e., they are motivated, active, } \\
\text { and consider themselves to be responsible elements of } \\
\text { the project. }\end{array}$ & $\mathbf{1}$ & $\mathbf{2}$ & $\mathbf{3}$ & $\mathbf{4}$ & $\mathbf{5}$ & $\mathbf{X}$ \\
\hline
\end{tabular}

Decision Time

We strive to make important project decisions rapidly

within short timeframes. 


\begin{tabular}{|l|c|c|c|c|c|c|}
\hline Team Distribution & & & & & \\
\hline $\begin{array}{l}\text { The members in our team are geographically closely } \\
\text { located. }\end{array}$ & 1 & 2 & 3 & 4 & 5 & $\mathbf{X}$ \\
\hline $\begin{array}{l}\text { The other teams that we interact with within or outside } \\
\text { our organization are geographically closely located to } \\
\text { ours. }\end{array}$ & 1 & 2 & 3 & 4 & 5 & $\mathbf{X}$ \\
\hline
\end{tabular}

\begin{tabular}{|l|c|c|c|c|c|c|}
\hline Team size & & & & & & \\
\hline $\begin{array}{l}\text { We work in small teams (no more than 20-40 members) } \\
\text { in our projects. }\end{array}$ & 1 & 2 & 3 & 4 & 5 & $\mathrm{x}$ \\
\hline
\end{tabular}

\begin{tabular}{|l|c|c|c|c|c|c|}
\hline Corporate culture & & & & & \\
\hline Our organization encourages rapid communication. & 1 & 2 & 3 & 4 & 5 & $\mathbf{X}$ \\
\hline Our organization has the culture for trusting people. & 1 & 2 & 3 & 4 & 5 & $\mathbf{X}$ \\
\hline $\begin{array}{l}\text { Our management has the culture for supporting the } \\
\text { decisions of the developers. }\end{array}$ & 1 & 2 & 3 & 4 & 5 & $\mathbf{X}$ \\
\hline Our organizational culture is customer centric. & 1 & 2 & 3 & 4 & 5 & $\mathbf{X}$ \\
\hline $\begin{array}{l}\text { Our organization encourages fast feedback from } \\
\text { customers }\end{array}$ & 1 & 2 & 3 & 4 & 5 & $\mathbf{X}$ \\
\hline Our organization encourages changing requirements. & 1 & 2 & 3 & 4 & 5 & $\mathbf{X}$ \\
\hline $\begin{array}{l}\text { Our organization does not have a bureaucratic } \\
\text { management structure. }\end{array}$ & 1 & 2 & 3 & 4 & 5 & $\mathbf{X}$ \\
\hline $\begin{array}{l}\text { Our management, developers, and testers are in total } \\
\text { agreement to use agile practices. }\end{array}$ & 1 & 2 & 3 & 4 & 5 & $\mathbf{X}$ \\
\hline
\end{tabular}

\begin{tabular}{|l|c|c|c|c|c|c|}
\hline Planning & & & & & & \\
\hline $\begin{array}{l}\text { Our software development team relies on internalized, } \\
\text { informal, undocumented plans (as against formal } \\
\text { documented plans). }\end{array}$ & 1 & 2 & 3 & 4 & 5 & $x$ \\
\hline
\end{tabular}

\begin{tabular}{|c|c|c|c|c|c|c|}
\hline \multicolumn{7}{|l|}{ Control } \\
\hline $\begin{array}{l}\text { Our software development team has qualitative control } \\
\text { (as against quantitative performance measures). }\end{array}$ & 1 & 2 & 3 & 4 & 5 & $\mathbf{x}$ \\
\hline \multicolumn{7}{|l|}{ Technical competency } \\
\hline $\begin{array}{l}\text { Our team generally consists of technically competent } \\
\text { and experienced people (who have developed alike } \\
\text { software in the past, have practical experience of the } \\
\text { technology domain). }\end{array}$ & 1 & 2 & 3 & 4 & 5 & $\mathbf{x}$ \\
\hline
\end{tabular}

\begin{tabular}{|c|c|c|c|c|c|c|}
\hline Personal characteristics & & & & & & \\
\hline $\begin{array}{l}\text { The majority of our team members have strong } \\
\text { interpersonal and communication skills. }\end{array}$ & 1 & 2 & 3 & 4 & 5 & $\mathbf{x}$ \\
\hline $\begin{array}{l}\text { The majority of the members of our team consists of } \\
\text { people who are honest. }\end{array}$ & 1 & 2 & 3 & 4 & 5 & $\mathbf{x}$ \\
\hline $\begin{array}{l}\text { The majority of the members of our team consists of } \\
\text { people who are motivated. }\end{array}$ & 1 & 2 & 3 & 4 & 5 & $x$ \\
\hline $\begin{array}{l}\text { The majority of the members of our team consists of } \\
\text { people who have collaborative attifude. }\end{array}$ & 1 & 2 & 3 & 4 & 5 & $\mathbf{x}$ \\
\hline $\begin{array}{l}\text { The majority of the members of our team consists of } \\
\text { people who are have sense of responsibility. }\end{array}$ & 1 & 2 & 3 & 4 & 5 & $\mathbf{x}$ \\
\hline $\begin{array}{l}\text { The majority of the members of our team consists of } \\
\text { people who have readiness to learn. }\end{array}$ & 1 & 2 & $\mathbf{3}$ & 4 & $\mathbf{5}$ & $\mathbf{x}$ \\
\hline
\end{tabular}




\begin{tabular}{|c|c|c|c|c|c|c|}
\hline \multicolumn{7}{|l|}{ Communication and Negotiation } \\
\hline $\begin{array}{l}\text { Our projects have mechanisms that enable personnel } \\
\text { to communicate and negotiate quickly and effectively } \\
\text { with developers, operations, support, customers, } \\
\text { management, and business areas. }\end{array}$ & 1 & 2 & 3 & 4 & 5 & $\mathbf{x}$ \\
\hline $\begin{array}{l}\text { In most cases, communication and negotiation in our } \\
\text { projects are face-to-face. }\end{array}$ & 1 & 2 & 3 & 4 & 5 & $x$ \\
\hline $\begin{array}{l}\text { In most cases, communication and negotiation in our } \\
\text { projects happen between people who are physically } \\
\text { close to one another. }\end{array}$ & 1 & 2 & 3 & 4 & 5 & $\mathbf{x}$ \\
\hline $\begin{array}{l}\text { In most cases, communication and negotiation in our } \\
\text { projects happen between people who work in the same } \\
\text { (similar) time zone as ours. }\end{array}$ & 1 & 2 & 3 & 4 & 5 & $x$ \\
\hline $\begin{array}{l}\text { Most people in our projects are amicable to each other } \\
\text { to such an extent that they communicate with each other } \\
\text { with trust and good will. }\end{array}$ & 1 & 2 & 3 & 4 & 5 & $\mathbf{x}$ \\
\hline
\end{tabular}

\begin{tabular}{|l|c|c|c|c|c|c|}
\hline Societal Culture & & & & & \\
\hline $\begin{array}{l}\text { The people of our country are in general } \\
\text { communicative. }\end{array}$ & 1 & 2 & 3 & 4 & 5 & $\mathbf{X}$ \\
\hline The people of our country are in general dynamic. & 1 & 2 & 3 & 4 & 5 & $\mathbf{X}$ \\
\hline $\begin{array}{l}\text { The people of our country in general have progressive } \\
\text { attitude. }\end{array}$ & 1 & 2 & 3 & 4 & 5 & $\times$ \\
\hline $\begin{array}{l}\text { The majority of the members of our team have similar } \\
\text { social culture, even though they might be belonging to } \\
\text { different nationalities and provinces. }\end{array}$ & 1 & 2 & 3 & 4 & 5 & $\times$ \\
\hline
\end{tabular}

\begin{tabular}{|l|c|c|c|c|c|c|}
\hline Training and Learning & & & & & & \\
\hline $\begin{array}{l}\text { Our team members are in general always willing to } \\
\text { continuously learn from one another and train them } \\
\text { through mentoring and professionally guided } \\
\text { discussions than through formal training. }\end{array}$ & $\mathbf{1}$ & $\mathbf{2}$ & $\mathbf{3}$ & $\mathbf{4}$ & $\mathbf{5}$ & $\mathbf{X}$ \\
\hline
\end{tabular}

You are invited to specify any additional factors that you believe impact the success of agile software development projects. Please rate your responses on a scale of 1 to 5 .

\begin{tabular}{|l|c|c|c|c|c|}
\hline & $\begin{array}{c}\text { Strongly } \\
\text { Believe }\end{array}$ & $\begin{array}{c}\text { Somewhat } \\
\text { Believe }\end{array}$ & Neutral & $\begin{array}{c}\text { Don't } \\
\text { Strongly } \\
\text { Disbelieve }\end{array}$ & $\begin{array}{c}\text { Strongly } \\
\text { Disbelieve }\end{array}$ \\
\hline Other factors & & & & & \\
\hline 1. & 1 & 2 & 3 & 4 & 5 \\
\hline 2. & 1 & 2 & 3 & 4 & 5 \\
\hline 3. & 1 & 2 & 3 & 4 & 5 \\
\hline 4. & 1 & 2 & 3 & 4 & 5 \\
\hline 5. & 1 & 2 & 3 & 4 & 5 \\
\hline
\end{tabular}


Please rate, on a scale of 1 to 5 , your assessment of the degree of impact of practicing agile software development in your projects.

\begin{tabular}{|c|c|c|c|c|c|c|}
\hline & $\begin{array}{l}\text { Strongly } \\
\text { Disagree }\end{array}$ & $\begin{array}{c}\text { Somewhat } \\
\text { Disagree }\end{array}$ & $\begin{array}{c}\text { Neither } \\
\text { Disagree } \\
\text { nor Agree }\end{array}$ & $\begin{array}{c}\text { Somewhat } \\
\text { Agree }\end{array}$ & $\begin{array}{c}\text { Strongly } \\
\text { Agree }\end{array}$ & $\begin{array}{c}\text { Not } \\
\text { Applicable } \\
\text { or Don't } \\
\text { know }(X)\end{array}$ \\
\hline Reduced delivery schedules. & 1 & 2 & 3 & 4 & 5 & $\bar{x}$ \\
\hline Increased retum on investment (ROI). & 1 & 2 & 3 & 4 & 5 & $\bar{x}$ \\
\hline $\begin{array}{l}\text { Increased flexibility to meet with the changing customer } \\
\text { requirements. }\end{array}$ & 1 & 2 & 3 & 4 & 5 & $\mathbf{X}$ \\
\hline Improved business processes. & 1 & 2 & 3 & 4 & 5 & $\mathbf{X}$ \\
\hline
\end{tabular}

\section{Changes and Challenges Survey}

The information collected from the survey questions below will enable us to enhance our understanding of the critical changes required and the challenges/risks involved in introducing agile software development practices in organizations practicing plan-driven (traditional) software development methodologies.

\section{CHANGES REQUIRED}

According to your view, are the following changes required for adopting agile software development practices in organizations practicing traditional, plan driven software development methodologies? Please rank the degree of importance of each.

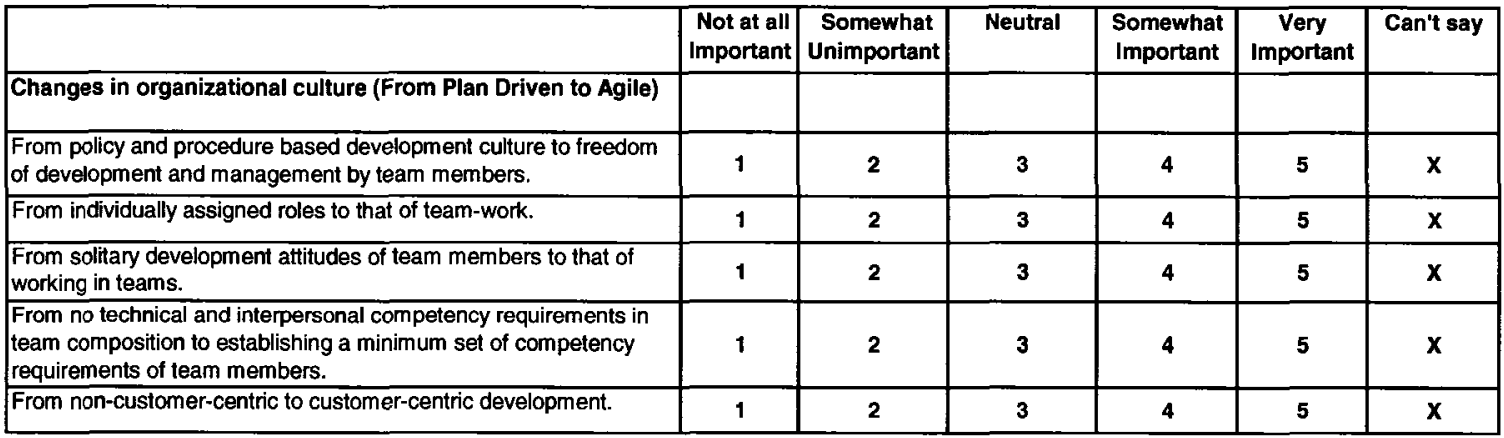

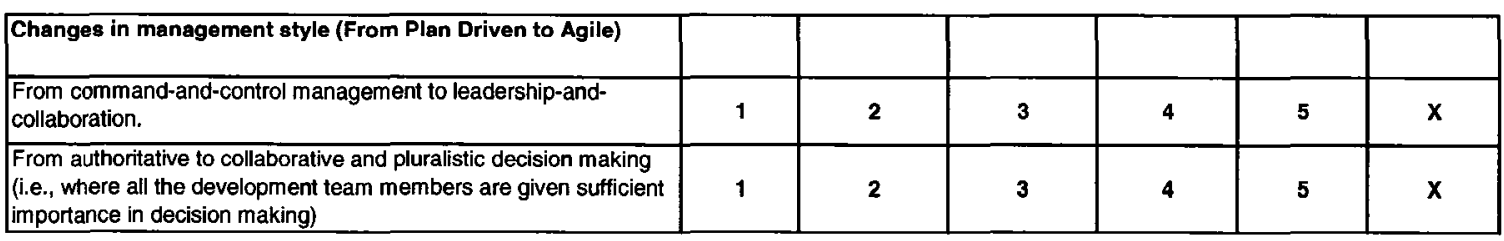

\begin{tabular}{|l|c|c|c|c|c|}
\hline $\begin{array}{l}\text { Changes in knowledge management strategy (From Plan } \\
\text { Driven to Agile) }\end{array}$ & & & & \\
\hline $\begin{array}{l}\text { From heavy documentation-based to tacit (not spoken) knowledge } \\
\text { management. }\end{array}$ & 1 & 2 & 3 & 4 & 5 \\
\hline
\end{tabular}

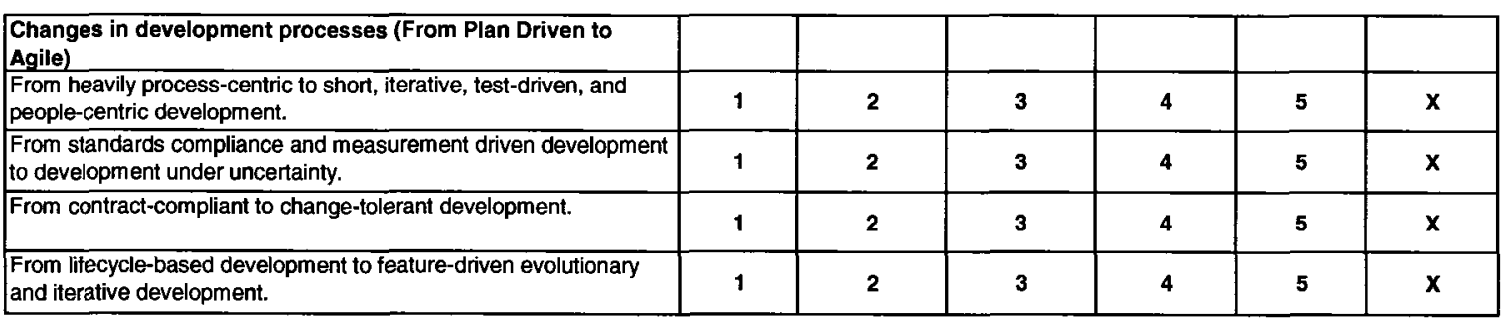


Please identify any other changes you feel are required for adopting agile software development practices in organizations practicing traditional, plan driven software development methodologies.

\begin{tabular}{|c|c|c|c|c|c|c|}
\hline & \begin{tabular}{|c|} 
Not at all \\
Important
\end{tabular} & \begin{tabular}{|c|} 
Somewhat \\
Unimportant
\end{tabular} & Neutral & $\begin{array}{l}\text { Somewhat } \\
\text { Important }\end{array}$ & $\begin{array}{c}\text { Very } \\
\text { Important }\end{array}$ & Can't say \\
\hline \multicolumn{7}{|c|}{ Other changes required (From Plan Driven to Agile) } \\
\hline 2. & 1 & 2 & 3 & 4 & 5 & $\mathbf{x}$ \\
\hline 4. & 1 & 2 & 3 & 4 & 5 & $\mathbf{x}$ \\
\hline 5. & 1 & 2 & 3 & 4 & 5 & $x$ \\
\hline
\end{tabular}

\section{CHALLENGES/ RISKS INVOLVED}

According to your view, rank the degree of importance of the challenges/risks involved in adopting agile software development practices in organizations practicing traditional, plan driven software development methodologies. Please rank their importance in a scale of 1 to 5 according to your views.

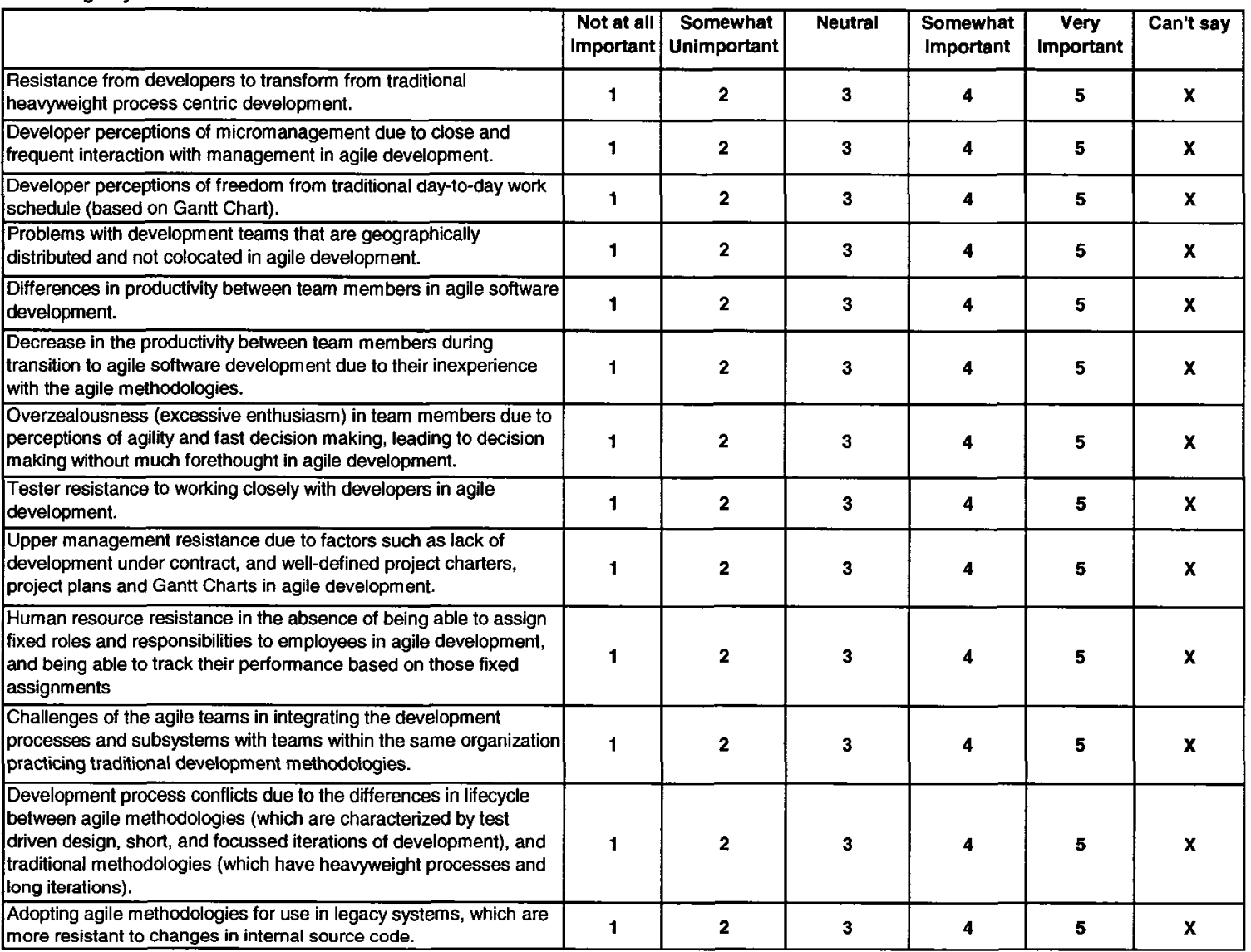




\begin{tabular}{|c|c|c|c|c|c|c|}
\hline $\begin{array}{l}\text { Differences in development processes between agile } \\
\text { methodologies (which are more informal requirements driven), and } \\
\text { traditional methodologies (which are more formal requirements } \\
\text { driven). }\end{array}$ & 1 & 2 & 3 & 4 & 5 & $\mathbf{x}$ \\
\hline $\begin{array}{l}\text { Continued conformance to traditional certified process standards } \\
\text { (such as CMM, and ISO) in organizations that have adopted them, } \\
\text { and are in the process of adopting agile methodologies. }\end{array}$ & 1 & 2 & 3 & 4 & 5 & $x$ \\
\hline $\begin{array}{l}\text { Differences in attitudes towards project success between the } \\
\text { management practicing traditional and agile methodologies. }\end{array}$ & 1 & 2 & 3 & 4 & 5 & $\mathbf{x}$ \\
\hline $\begin{array}{l}\text { Differences between the team size requirements and suitability of } \\
\text { the agile and traditional methodologies. }\end{array}$ & 1 & 2 & 3 & 4 & 5 & $\mathbf{x}$ \\
\hline $\begin{array}{l}\text { Problems with selecting the appropriate agile methodology and the } \\
\text { supporting tools according to organizational needs and } \\
\text { characteristics. }\end{array}$ & 1 & 2 & 3 & 4 & 5 & $\mathbf{x}$ \\
\hline
\end{tabular}

Please identify any other challenges / risks you feel are involved in adopting agile software development practices in organizations practicing traditional, plan driven software development methodologies. Please rank their importance in a scale of 1 to 5 according to your views.

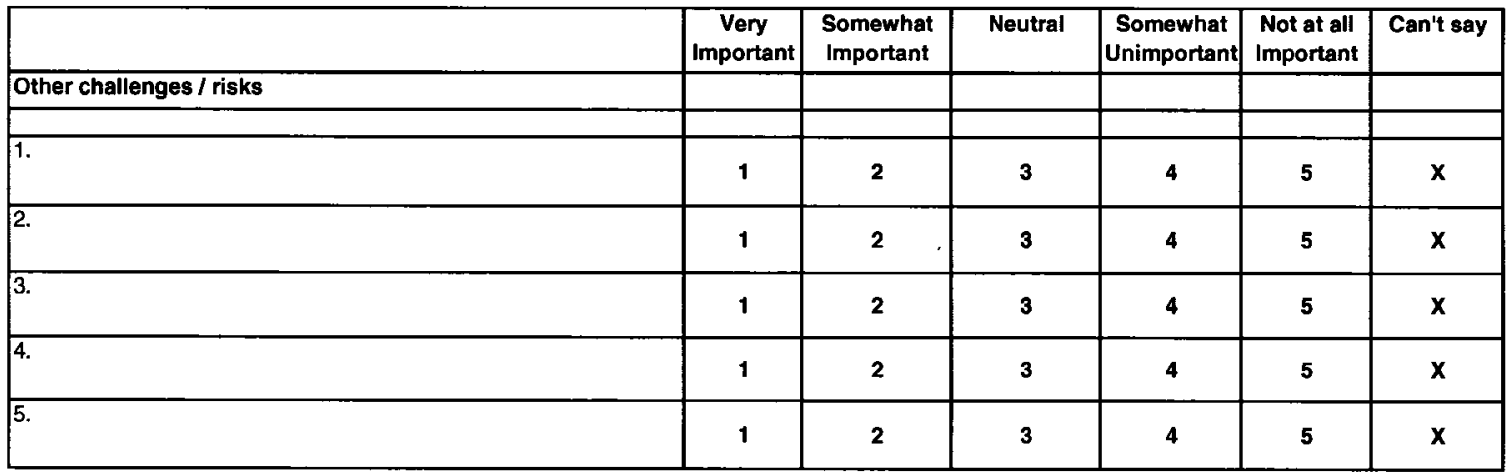




\section{Appendix C}

\section{Labels of Variables Used in the Study}

In this Appendix, we list the labels/codes that were assigned to the different variables used in this study. These labels are then used throughout during statistical analysis using SPSS, and also while presenting the results of statistical analysis.

\section{C.1. Variables used in Research Question 1}

In this Section, the different independent and dependent variables used in the Success Factors survey and their corresponding labels are listed.

Table C.8.1: Variables used in Research Question 1

\begin{tabular}{|l|l|}
\hline \multicolumn{1}{|c|}{ Variables } & \multicolumn{1}{|c|}{ Label } \\
\hline Customer Satisfaction & Ind1 \\
\hline Customer Collaboration & Ind2 \\
\hline Customer Commitment & Ind3 \\
\hline Decision Time & Ind4 \\
\hline Team Distribution & Ind5 \\
\hline Team size & Ind6 \\
\hline Corporate culture & Ind7 \\
\hline Planning & Ind8 \\
\hline Control & Ind9 \\
\hline Technical competency & Ind10 \\
\hline Personal characteristics & Ind11 \\
\hline Communication and Negotiation & Ind12 \\
\hline Societal Culture & Ind13 \\
\hline Training and Learning & Ind14 \\
\hline Reduced delivery schedules. & D1 \\
\hline & \\
\hline
\end{tabular}




\begin{tabular}{|l|l|}
\hline Increased return on investment (ROI). & D2 \\
\hline Increased ability to meet with the current customer requirements. & D3 \\
\hline $\begin{array}{l}\text { Increased flexibility to meet with the changing customer } \\
\text { requirements. }\end{array}$ & D4 \\
\hline Improved business processes. & D5 \\
\hline Success & Dependent \\
\hline
\end{tabular}

In cases where the independent variables are measured using multiple sub-questions, those subquestions are represented as $a, b, c, \ldots$ etc. in the same sequence as they appear in the survey questionnaire in Appendix B.

\section{C.2. Variables used in Research Question 2}

In this Section, the different change item variables used in the Changes survey and their corresponding labels are listed.

Table C.8.2: Variables used in Research Question 2

\begin{tabular}{|l|c|}
\hline \multicolumn{1}{|c|}{ Variables } & Label \\
\hline Changes in organizational culture (From Plan Driven to Agile) & $\mathrm{C} 1$ \\
\hline $\begin{array}{l}\text { From policy and procedure based development culture to freedom } \\
\text { of development and management by team members. }\end{array}$ & $\mathrm{C} 1.1$ \\
\hline From individually assigned roles to that of team-work. & $\mathrm{C} 1.2$ \\
\hline $\begin{array}{l}\text { From solitary development attitudes of team members to that of } \\
\text { working in teams. }\end{array}$ & $\mathrm{C} 1.3$ \\
\hline $\begin{array}{l}\text { From no technical and interpersonal competency requirements in } \\
\text { team composition to establishing a minimum set of competency } \\
\text { requirements of team members. }\end{array}$ & $\mathrm{C} 1.4$ \\
\hline From non-customer-centric to customer-centric development. & $\mathrm{C} 1.5$ \\
\hline Changes in management style (From Plan Driven to Agile) & $\mathrm{C} 2$ \\
\hline $\begin{array}{l}\text { From command-and-control management to leadership-and- } \\
\text { collaboration. }\end{array}$ & $\mathrm{C} 2.1$ \\
\hline $\begin{array}{l}\text { From authoritative to collaborative and pluralistic decision making } \\
\text { (i.e., where all the development team members are given sufficient } \\
\text { importance in decision making) }\end{array}$ & $\mathrm{C} 2.2$ \\
\hline $\begin{array}{l}\text { Changes in knowledge management strategy (From Plan Driven to } \\
\text { Agile) }\end{array}$ & $\mathrm{C} 3$ \\
\hline $\begin{array}{l}\text { From heavy documentation-based to tacit (not spoken) knowledge } \\
\text { management. }\end{array}$ & $\mathrm{C} 3.1$ \\
\hline
\end{tabular}




\begin{tabular}{|l|c|}
\hline Changes in development processes (From Plan Driven to Agile) & $\mathrm{C} 4$ \\
\hline $\begin{array}{l}\text { From heavily process-centric to short, iterative, test-driven, and } \\
\text { people-centric development. }\end{array}$ & $\mathrm{C} 4.1$ \\
\hline $\begin{array}{l}\text { From standards compliance and measurement driven } \\
\text { development to development under uncertainty. }\end{array}$ & $\mathrm{C} 4.2$ \\
\hline From contract-compliant to change-tolerant development. & $\mathrm{C} 4.3$ \\
\hline $\begin{array}{l}\text { From lifecycle-based development to feature-driven evolutionary } \\
\text { and iterative development. }\end{array}$ & $\mathrm{C} 4.4$ \\
\hline
\end{tabular}

\section{C.3. Variables used in Research Question 3}

In this Section, the different challenge item variables used in the Challenges survey and their corresponding labels used in are listed.

Table C.8.3: Variables Used in Research Question 3

\begin{tabular}{|l|c|}
\hline \multicolumn{1}{|c|}{ Variables } & Label \\
\hline $\begin{array}{l}\text { Resistance from developers to transform from traditional } \\
\text { heavyweight process centric development. }\end{array}$ & Chal1 \\
\hline $\begin{array}{l}\text { Developer perceptions of micromanagement due to close and } \\
\text { frequent interaction with management in agile development. }\end{array}$ & Chal2 \\
\hline $\begin{array}{l}\text { Developer perceptions of freedom from traditional day-to-day } \\
\text { work schedule (based on Gantt Chart). }\end{array}$ & Chal3 \\
\hline $\begin{array}{l}\text { Problems with development teams that are geographically } \\
\text { distributed and not colocated in agile development. }\end{array}$ & Chal4 \\
\hline $\begin{array}{l}\text { Differences in productivity between team members in agile } \\
\text { software development. }\end{array}$ & Chal6 \\
\hline $\begin{array}{l}\text { Decrease in the productivity between team members during } \\
\text { transition to agile software development due to their inexperience } \\
\text { with the agile methodologies. }\end{array}$ & Chal7 \\
\hline $\begin{array}{l}\text { Overzealousness (excessive enthusiasm) in team members due to } \\
\text { perceptions of agility and fast decision making, leading to decision } \\
\text { making without much forethought in agile development. }\end{array}$ & Chal8 \\
\hline $\begin{array}{l}\text { Tester resistance to working closely with developers in agile } \\
\text { development. }\end{array}$ & Chal9 \\
\hline $\begin{array}{l}\text { Upper management resistance due to factors such as lack of } \\
\text { development under contract, and well-defined project charters, } \\
\text { project plans and Gantt Charts in agile development. }\end{array}$ & $\begin{array}{l}\text { Chal10 } \\
\text { Human resource resistance in the absence of being able to assign } \\
\text { fixed roles and responsibilities to employees in agile development, } \\
\text { and being able to track their performance based on those fixed } \\
\text { assignments }\end{array}$ \\
\hline \begin{tabular}{l} 
Challenges of the agile teams in integrating the development \\
\hline
\end{tabular} & Chal11 \\
\hline
\end{tabular}


processes and subsystems with teams within the same organization practicing traditional development methodologies.

Development process conflicts due to the differences in lifecycle between agile methodologies (which are characterized by test driven design, short, and focused iterations of development), and traditional methodologies (which have heavyweight processes and long iterations).

Adopting agile methodologies for use in legacy systems, which are more resistant to changes in internal source code.

Differences in development processes between agile methodologies (which are more informal requirements driven), and traditional methodologies (which are more formal requirements driven).

Differences in performance management approaches in traditional methodologies (which are more contract and milestone driven), and agile methodologies (which are based on incorporating continuous customer feedback into the development lifecycle.) Continued conformance to traditional certified process standards (such as CMM, and ISO) in organizations that have adopted them, and are in the process of adopting agile methodologies.

Differences in attitudes towards project success between the management practicing traditional and agile methodologies. Differences between the team size requirements and suitability of the agile and traditional methodologies.

Problems with selecting the appropriate agile methodology and the supporting tools according to organizational needs and characteristics. 


\section{Appendix D \\ Feedback to Open-Ended Questions}

In this Appendix, we list the feedback obtained to some of the open-ended questions from the surveys. The lists below are dumped "as is" from what was obtained from the survey responses. A visual inspection of all these lists reveal that many of them can be classified to belong to the major success factors, changes, and challenges listed as closed-ended questions in the survey questionnaire.

\section{D.1. List of Other Success Factors Obtained from Open-Ended Questions}

We list below the other success factors that were suggested by different respondents through the open questions in the Success Factors survey.

- Customer must participate effectively in process and be able to balance business and technical goals. If not, you are doomed to fail over and over... or at minimum do Agile very badly.

- Understanding of what failed before

- Mentoring and training

- Pair Programming: designs are discussed, knowledge is transferred between members

- Business Commitment to process

- Buy in to agile practices, especially from management

- Talent

- Open and continuous feedback between team members

- Time Boxing is probably the most important thing 
- Involvement from customer

- Management Support from the highest level

- Realizing the service-providing role over specification-demanding

- Time to market

- Testing

- Are your team members full-time on this project?

- We integrate code constantly.

- Team dynamic \& Team development

- Organizational management understands and supports agile values and principles

- Adaptability

- My developers know the business and can sometimes be their own customers

- Flexibility: Not all the practices are adopted/used religiously, but rewards are still evident.

- Top management commitment to agile practices

- Having intellect

- We have weekly showcases to show progress to customer

- Simple communication tools like wiki

- Our customers are technically adapt

- Staff changes through attrition or reorganizing

- How mature the management of our organisation is in the field of agile development 
- Scale down and specify the requirements so that it can be delivered in a short time.

- Full time Agile coach(es)

- Understanding of the process by the customer

- Progress Visibility (completed story points per iteration) for everyone - including customer.

- Test Driven Development

- Organizational buy-in and support of the processes

- Management infrastructure in place

- Agile contract

- Information radiators for communication (charts and cards on the wall)

- We sometimes use non-agile methodologies in order to satisfy timelines.

- Smart people

- Good Tool

- Reflection

- Pair Programming

- Type of Application Deliverable (In-House IT project vs Shrink Wrap Software)

- Agile Specific Project Management Tooling (Rally)

- Everyone (including data people, QA people, ...) is agile

- Taking up responsibility

- Having a base of good principles

- Team empowerment 
- Maturity of the team members

- Use of a common information radiator

- Goal-driven functionality and UI design

- Technical support environment

- Use of ProjectCards to organize the project

- Criticality of the software - what is at stake?

- Disciplined yet light processes

- Continuous integration \& governance tools are needed

- Willingness of programmers to work as pairs. Is difficult among experienced developers?

- Anxiety

- Formal contracts

- Team workings towards same known goal

- The client organization having incentives that are not contradicting the use of agile methods

- Analysis of what our core problems are

- Permission to do quality work

- Automated Testing: allows for shared code ownership (developers can feel free to make changes, having unit/functional/integration tests as safeguards)

- Quantitative demonstration of benefits of agile (DSDM) - independent metrics case study

- Root cause analysis, if something goes wrong 
- Getting the team test infected using xUnit and some kind of mocking tool

- Individual capabilities of team members

- Process transparency to key stakeholders

- Not working on systems with closed list of modules

- Continuous feedback

- Are your team members experienced with agile development practices?

- We write unit tests before writing code

- Communication rules

- Passionate

- Our customers trust our developers to add new features without their consent

- People have other day jobs to do at same time doing project

- Constant collaboration with customer

- Team believe in the motivation behind agile practices

- How mature the client is (some just do not want to cooperate - they try to buy software like groceries)

- Full time iteration manager (internally-facing project manager)

- Integrity of estimations

- Open and honest about the project status - good or bad to recognize problems earlier.

- Use of continuous integration

- Frequent customer visits (not co-located) and telecons

- Stakeholder buyin 
- Pair programming (constant code review)

- Trusting customers

- Training

- Planning Game

- Outside Coaching Services (Rally)

- Establishing the agile value system is more important than following pre-defined practices

- Frequent delivery of used software

- Significant Business Commitment

- CMM Background

- Developer familiarity with the project model

- Craftsmanship

- You need buy-in from customer

- Complexity of the software

- Disciplined yet light documentation

- Refactoring the code often

- Agile approach differs among business units.

- Customer-Vendor relationship

- Having a competent, committed and authorized product owner/customer

- Refactoring

- Track record with client

- Not being a perfectionist 
- Mocks are important, if you can't mock, you can't unit test

- Prioritisation of all development activities decided by the business

- Are your developers experienced?

- We have a weekly "show and tell" for our customers: strongly agree.

- Self-criticism

- Business priorities change, conflicts between availability of business versus IT technical staff

- Ability for test team to adapt is essential

- UI should develop along with the software

- Design architecture stable

- Lack of politics

- Test driven development (ensures quality and no unnecessary code)

- Strong estimation

- Courage to tell the truth about project

- Domain knowledge (not technology)

- Risk sharing contracts with suppliers

- Distributed agile development

- Developer familiarity with the technical platform

- Time to delivery

- Developer skills

- Business driven not IT/IM/IS led

- We estimate task duration and have the customer choose tasks \& priorities 
- Task orientation

- Respect of individual

- Skilled developers

- Good People

- Daily Standup

- Lightweight written plans (like burndown charts)

- Proper usage of tools

- Dynamism of the project - how many changes in requirements per month

- Select a strong Agile partner when getting started

- (Known) architecture

- Documented Process NOT JDI!

- Paired programming is a critical success factor in keeping the teams up to speed on development issues, and cross training.

- We program in pairs: strongly agree.

- Humility

- Fun

- The major benefit of agility is to reduce risk bby knowing about the real status of the project

- The goal isn't to be agile: It's to be effective.

- Command and control way of working

- Stability of team

- Domain knowledge 


\section{D.2. List of Other Changes Obtained from Open-Ended Questions}

We list below the other change items that were suggested by different respondents through the open questions in the Changes survey.

- Permission from management to make the change

- Shared team room

- Unit Testing, mocks, and continuous integration

- Knowledge sharing

- The ability to reject structured documentation, such as a survey instrument, when it doesn't help my client

- Early involvement of business analysts (Menlo Innovations calls them "High Tech Anthropologists").

- Openness management

- Trust your developers

- The information about agile methodologies. If one person does not have the knowledge about agile development and is unwilling to learn, he can be a real stooper for the project. (management included) .

- Test-driven development (automated tests specify the behaviour)

- Education

- Pair Programming to foster learning is very important

- Increase of trust that seems to be lacking within a lot of organizations

- Size of teams kept small (beck etc.) 
- Flexibility of customers

- Business sponsor responsibility for prioritisation and release planning

- Honesty in communication

- Test Driven Development

- Agile specific tooling for collaboration, planning and tracking

- Shift in management thinking

- From predict to adaptive

- From large-scale scope management to continuous (micro) scope management

- Management acceptance of risk

- Test Driven Development

- Away with micromanagement

- Open communication

- Individual motivation to make changes

- Sales skills in the technical ranks

- Weekly releases as soon as the project begins.

- A right to errors culture

- Make sure your developers know your business

- Continuous integration / shared code base

- Architects develop alongside team

- Trust by customers

- Take small steps

- Continuous builds 
- Drive to Agile has to come from within the team - allow team to control not managers dictating method

- Scope of projects should be reduced

- Management acceptance of uncertainty

- Continuous build

- Programmers shouldn't have to justify each task, but deliver working software

- Proper development team environment

- Involvement in team activities

- Functional test development in parallel with code.

- Courageous

- Collective ownership of the solution

- More decisions through prototyping (spikes) and willingness to throw away these spikes

- Lack of focus on progress measurement can be a disaster

- Developers earning that trust

- Automated Tests

- Willingness to negotiate

- Paired programming

- Clear project information radiators

- Development teams assigned prioritized tasks with NO OTHER EXPECTATION.

- Trust 
- Early, continuous feedback (from customers, QA, automated tests)

- Viewing agile as different from "hacking"

- Continuous communication

- Honesty and integrity in communication about the development team progress (or lack thereof)

- Tenacity

\section{D.3. List of Other Challenges Obtained from Open-Ended Questions}

We list below the other challenges that were suggested by different respondents through the open questions in the Challenges survey.

- Fear of change from team members

- Getting the customer to buy into it

- Those experienced in traditional methodologies feeling threatened when the company adopts an agile methodology.

- Ability to engage the customer every iteration as opposed to every few months or every release.

- Perception that this is something "new" and needs to be "tried out" first.

- Focus on development not deployment of system

- Middle level management resistance

- Developer resistance to working with testers

- Documentation makes people feel secure

- Requirements management process 
- Non-technically driven purchases of software products that are hard to test and integrate

- Difficulty in communicating project status for Agile process

- Different conceptions of how the team should be working. Some people pushing, others resisting.

- Getting developers to accept TDD

- Lack of metric

- Still requires good system testing

- Management desire for fixed price, fixed scope

- Some people don't like the additional responsibility of an agile project

- Finding a good PM who can be flexible

- Lack of success demonstration

- Perceived lack of documentation

- Business users sometimes find it difficult to accept we don't deliver bells and whistles

- Unrequired accompaniment of team member

- Delivery of "almost-ready" code

- Some people find it difficult to adapt to constant change

- Seeing agile as a silver bullet

- Patchy quality of testing 


\section{Appendix E \\ Additional Statistical Results}

In this Appendix, we present the detailed statistical analysis results that were obtained

while analyzing the three research questions considered in our study.

\section{E.1. Research Question 1: Consolidated Dependent Variable Success}

The detailed statistical analysis results using the consolidated dependent variable Success

are presented in this Section.

\section{E1.1. Detailed Descriptive Statistical Results}

Table E.1: Research Question 1: Detailed Descriptive Statistical Results

\begin{tabular}{|c|c|c|c|c|c|c|c|c|c|c|}
\hline \multicolumn{11}{|c|}{ Descriptive Statistics } \\
\hline & $\mathbf{N}$ & Range & Minimum & Maximum & Mean & Std. & Skey & mess & Kur & \\
\hline & Statistic & Statistic & Statistic & Statistic & Statistic & Statistic & Statistic & Std. Error & Statistic & Sid. Error \\
\hline Ind 1 & 174 & 5 & 0 & 5 & 4.76 & .595 & -4.192 & .184 & 25.669 & .366 \\
\hline Ind 2 & 174 & 5 & 0 & 5 & 4.17 & .925 & -1.357 & .184 & 2.224 & .366 \\
\hline Ind 3 & 174 & 5 & 0 & 5 & 3.95 & 1.074 & -1.210 & .184 & 1.506 & .366 \\
\hline ind 4 & 173 & 5 & 0 & 5 & 4.30 & 815 & -1.452 & .185 & 3.803 & .367 \\
\hline Ind 5 & 174 & 4 & 1 & 5 & 3.22 & 1.267 & .220 & .184 & -.992 & .366 \\
\hline Ind 6 & 174 & 5 & 0 & 5 & 4.64 & .899 & -3.044 & .184 & 9.511 & .366 \\
\hline Ind 7 & 174 & 4 & 1 & 5 & 4.16 & .793 & -1.377 & .184 & 1.621 & .366 \\
\hline Ind 8 & 174 & 5 & 0 & 5 & 3.70 & 1.227 & -.873 & .184 & -.050 & .366 \\
\hline Ind 9 & 174 & 5 & 0 & 5 & 3.60 & 1.501 & -1.157 & .184 & .463 & .366 \\
\hline Ind 10 & 173 & 4 & 1 & 5 & 4.11 & .985 & -1.109 & .185 & .758 & .367 \\
\hline Ind 11 & 174 & $\mathbf{3}$ & 2 & 5 & 4.39 & .577 & -1.304 & .184 & 2.130 & .366 \\
\hline Ind 12 & 173 & 3 & 2 & 5 & 4.02 & .748 & -.551 & .185 & -.462 & .367 \\
\hline Ind 13 & 172 & 5 & 0 & 5 & 3.75 & 1.001 & -1.086 & .185 & 1.829 & .368 \\
\hline Ind 14 & 171 & 4 & 1 & 5 & 4.34 & .783 & -1.273 & .186 & 1.950 & .369 \\
\hline Dependent & 173 & 2 & 3 & 5 & 4.37 & .557 & -.871 & .185 & .079 & .367 \\
\hline Ind 7a & 174 & 3 & 2 & 5 & 4.51 & .831 & -1.749 & .184 & 2.276 & .366 \\
\hline Ind $7 b$ & 174 & 4 & 1 & 5 & 4.36 & .956 & -1.626 & .184 & 2.170 & .366 \\
\hline Ind $7 c$ & 174 & 5 & 0 & 5 & 4.22 & 1.069 & -1.624 & .184 & 2.343 & .366 \\
\hline Ind 7d & 174 & 5 & 0 & 5 & 4.15 & .980 & -1.349 & .184 & 2.019 & .366 \\
\hline Ind $7 \theta$ & 173 & 5 & 0 & 5 & 4.27 & 1.046 & -1.643 & .185 & 2.357 & .367 \\
\hline Ind $7 f^{\prime}$ & 174 & 4 & 1 & 5 & 3.87 & 1.163 & -.832 & .184 & -.271 & .366 \\
\hline Ind 79 & 174 & 4 & 1 & 5 & 3.94 & 1.363 & -1.045 & .184 & .294 & .366 \\
\hline Ind 7h & 174 & 5 & 0 & 5 & 3.95 & 1.192 & -1.093 & .184 & .524 & .366 \\
\hline Ind 11a & 174 & 4 & 1 & 5 & 3.94 & .878 & -.768 & .184 & .357 & .366 \\
\hline Ind 11b & 174 & 2 & 3 & 5 & 4.64 & .568 & -1.348 & .184 & .857 & .366 \\
\hline Ind 11c & 174 & 4 & 1 & 5 & 4.43 & .732 & -1.503 & .184 & 3.150 & .366 \\
\hline Ind 11d & 174 & 3 & 2 & 5 & 4.37 & .755 & -1.149 & .184 & 1.055 & .366 \\
\hline Ind 110 & 174 & 5 & 0 & 5 & 4.47 & .795 & -2.244 & .184 & 7.509 & .366 \\
\hline Ind $11 f$ & 174 & 4 & 1 & 5 & 4.47 & .830 & -1.727 & .184 & 2.796 & .366 \\
\hline Ind 12a & 173 & 5 & 0 & 5 & 4.13 & 1.000 & $-1,436$ & .185 & 2.360 & .367 \\
\hline Ind 12b & 173 & 4 & 1 & 5 & 3.94 & 1.204 & -.979 & .185 & -.139 & .367 \\
\hline Ind $12 \mathrm{c}$ & 172 & 4 & 1 & 5 & 3.79 & 1.267 & -.786 & .185 & -.610 & .368 \\
\hline Ind 12d & 172 & 5 & 0 & 5 & 3.90 & 1.284 & -.942 & .185 & -.267 & 368 \\
\hline Ind $12 \theta$ & 172 & 4 & 1 & 5 & 4.32 & .829 & -1.719 & .185 & 4.302 & .368 \\
\hline Ind 13a & 172 & 5 & 0 & 5 & 3.79 & 1.244 & -1.349 & .185 & 1.665 & .368 \\
\hline Ind $13 b$ & 172 & 5 & 0 & 5 & 3.69 & 1.309 & -1.284 & .185 & 1.385 & .368 \\
\hline ind $13 \mathrm{c}$ & 172 & 5 & 0 & 5 & 3.63 & $\$ .402$ & -1.206 & .185 & .913 & .368 \\
\hline ind 13 d & 172 & 5 & 0 & 5 & 3.89 & 1.192 & -1.210 & .185 & 1.276 & .368 \\
\hline Dop 1 & 173 & 5 & o & 5 & 4.14 & 1.002 & -1.476 & .185 & 2.730 & .387 \\
\hline Dep 1 & 172 & 5 & 0 & 5 & 4.24 & 1.059 & -1.968 & .185 & 4.705 & .368 \\
\hline Dep 3 & 173 & $\mathbf{3}$ & 2 & 5 & 4.65 & .626 & -1.867 & .185 & 3.435 & .367 \\
\hline Dep 4 & 173 & 3 & 2 & 5 & 4.75 & .544 & -2.505 & .185 & 7.433 & .367 \\
\hline Dep 5 & 173 & 5 & 0 & 5 & 4.08 & 1.078 & -1.670 & .185 & 3.844 & .367 \\
\hline ind $5 a$ & 174 & 4 & 1 & 5 & 3.78 & 1.494 & .798 & .184 & -.947 & .366 \\
\hline Ind 5b & 170 & 5 & 0 & 5 & 2.61 & 1.520 & .210 & .186 & -1.219 & .370 \\
\hline Valid N (listwise) & 159 & & & & & & & & & \\
\hline
\end{tabular}




\section{E1.2. Inter-Item Correlation Matrices for Variables Measured with Multiple Items}

Table E.2: Research Question 1: Inter-Item Correlation Matrix for Items in Ind7

\begin{tabular}{|l|r|r|r|r|r|r|r|r|}
\hline & \multicolumn{1}{|c|}{ Ind 7a } & \multicolumn{1}{c|}{ Ind 7b } & Ind 7c & \multicolumn{1}{c|}{ Ind 7d } & Ind 7e & Ind 7f & Ind 7g & Ind 7h \\
\hline Ind 7a & 1.000 & .721 & .470 & .356 & .523 & .483 & .687 & .412 \\
Ind 7b & .721 & 1.000 & .657 & .342 & .443 & .409 & .646 & .443 \\
Ind 7c & .470 & .657 & 1.000 & .426 & .467 & .365 & .466 & .373 \\
Ind 7d & .356 & .342 & .426 & 1.000 & .632 & .424 & .365 & .262 \\
Ind 7e & .523 & .443 & .467 & .632 & 1.000 & .529 & .590 & .357 \\
Ind 7f & .483 & .409 & .365 & .424 & .529 & 1.000 & .548 & .385 \\
Ind 7g & .687 & .646 & .466 & .365 & .590 & .548 & 1.000 & .445 \\
Ind 7h & .412 & .443 & .373 & .262 & .357 & .385 & .445 & 1.000 \\
\hline
\end{tabular}

Table E.0.3: Research Question 1: Inter-Item Correlation Matrix for Items in Ind11

\begin{tabular}{|l|r|r|r|r|r|r|}
\hline & \multicolumn{1}{|c|}{ Ind 11a } & \multicolumn{1}{c|}{ Ind 11b } & \multicolumn{1}{c|}{ Ind 11c } & \multicolumn{1}{c|}{ Ind 11d } & Ind 11e & \multicolumn{1}{c|}{ Ind 11f } \\
\hline Ind 11a & 1.000 & .444 & .375 & .492 & .405 & .408 \\
Ind 11b & .444 & 1.000 & .553 & .477 & .407 & .438 \\
Ind 11c & .375 & .553 & 1.000 & .659 & .518 & .621 \\
Ind 11d & .492 & .477 & .659 & 1.000 & .468 & .576 \\
Ind 11e & .405 & .407 & .518 & .468 & 1.000 & .507 \\
Ind 11f & .408 & .438 & .621 & .576 & .507 & 1.000 \\
\hline
\end{tabular}

Table E.4: Research Question 1: Inter-Item Correlation Matrix for Items in Ind12

\begin{tabular}{|l|r|r|r|r|r|}
\hline & \multicolumn{1}{|c|}{ Ind 12a } & \multicolumn{1}{c|}{ Ind 12b } & \multicolumn{1}{c|}{ Ind 12c } & \multicolumn{1}{c|}{ Ind 12d } & \multicolumn{1}{c|}{ Ind 12e } \\
\hline Ind 12a & 1.000 & .237 & .166 & -.031 & .201 \\
Ind 12b & .237 & 1.000 & .728 & .535 & .141 \\
Ind 12c & .166 & .728 & 1.000 & .701 & .091 \\
Ind 12d & -.031 & .535 & .701 & 1.000 & .064 \\
Ind 12e & .201 & .141 & .091 & .064 & 1.000 \\
\hline
\end{tabular}

Table E.5: Research Question 1: Inter-Item Correlation Matrix for Items in Ind13

\begin{tabular}{|l|r|r|r|r|}
\hline & \multicolumn{1}{|c|}{ Ind 13a } & \multicolumn{1}{|c|}{ Ind 13b } & \multicolumn{1}{c|}{ Ind 13c } & \multicolumn{1}{c|}{ Ind 13d } \\
\hline Ind 13a & 1.000 & .760 & .590 & .252 \\
Ind 13b & .760 & 1.000 & .656 & .276 \\
Ind 13c & .590 & .656 & 1.000 & .278 \\
Ind 13d & .252 & .276 & .278 & 1.000 \\
\hline
\end{tabular}

248 
Table E.6: Research Question 1: Inter-Item Correlation Matrix for Items in Dependent

\begin{tabular}{|l|r|r|r|r|r|}
\hline & \multicolumn{1}{|c|}{ Dep 1 } & \multicolumn{1}{|c|}{ Dep 1 } & \multicolumn{1}{c|}{ Dep 3 } & \multicolumn{1}{c|}{ Dep 4 } & \multicolumn{1}{c|}{ Dep 5 } \\
\hline Dep 1 & 1.000 & .517 & .290 & .320 & .315 \\
Dep 1 & .517 & 1.000 & .346 & .248 & .374 \\
Dep 3 & .290 & .346 & 1.000 & .669 & .306 \\
Dep 4 & .320 & .248 & .669 & 1.000 & .286 \\
Dep 5 & .315 & .374 & .306 & .286 & 1.000 \\
\hline
\end{tabular}

\section{E.1.3. Detailed Regression Analysis Tables}

Here we list those regression analysis tables that were not presented in the Data Analysis Chapter. 
Table E.7: Research Question 1: Detailed Correlations Results

\begin{tabular}{|c|c|c|c|c|c|c|c|c|c|c|c|c|c|c|c|c|}
\hline \multicolumn{17}{|c|}{ Correlations } \\
\hline & & Dep. & Ind 1 & $\operatorname{Ind} 2$ & $\operatorname{lnd} 3$ & Ind 4 & Ind 5 & $\operatorname{Ind} 6$ & $\operatorname{lnd} 7$ & $\operatorname{Ind} 8$ & Ind 9 & Ind 10 & $\operatorname{lnd} 11$ & Ind 12 & Ind 13 & $\operatorname{lnd} 14$ \\
\hline \multirow[t]{15}{*}{ Pearson Correlation } & Dep. & 1.000 & .140 & .232 & .253 & .254 & .074 & .097 & .241 & .075 & .301 & .102 & & & & \\
\hline & Ind 1 & .140 & 1.000 & .396 & .309 & .101 & .072 & -050 & .259 & -.103 & .158 & -.033 & .050 & -.002 & .023 & -.008 \\
\hline & Ind 2 & .232 & .396 & 1.000 & .562 & .238 & .070 & $\begin{array}{l}-.035 \\
-030\end{array}$ & .321 & -.048 & .139 &. .103 & .050 & .179 & .030 & .048 \\
\hline & $\operatorname{lnd} 3$ & .253 & .309 & .562 & 1.000 & .252 & .056 & .068 & .342 & -.089 & .106 & -.149 & -.076 & .158 & -.028 & -.031 \\
\hline & $\operatorname{lnd} 4$ & .254 & .101 & .238 & .252 & 1.000 & .098 & .165 & .456 & .129 & .250 & -.015 & .052 & .213 & .123 & .181 \\
\hline & Ind 5 & .074 & -.072 & .070 & .056 & .098 & 1.000 & .228 & .146 & .051 & .074 & -.074 & .017 & .573 & .050 & .099 \\
\hline & Ind 6 & . 097 & -.050 & -.035 & -.068 & . 165 & .228 & 1.000 & .039 & .106 & .066 & .092 & .130 & 193 & .041 & .066 \\
\hline & Ind 7 & .241 & .259 & .321 & .342 & .456 & .146 & .039 & 1.000 & .196 & . 186 & -.022 & .275 & .310 & .005 & .271 \\
\hline & Ind $B$ & .075 & -.103 & -.048 & -.089 & .129 & .051 & .106 & .196 & 1.000 & .238 & .218 & . 170 & .142 & -.043 & .125 \\
\hline & Ind 9 & .301 & .158 & .139 & .106 & .250 & .074 & .066 & .186 & .238 & 1.000 & .132 & .133 & .051 & .127 & .097 \\
\hline & Ind 10 & .102 & -.033 & -.103 & -.149 & .015 & -.074 & .092 & -.022 & .218 & .132 & 1.000 & .384 & .041 & .065 & .339 \\
\hline & Ind 11 & . 180 & .050 & .050 & -.076 & .052 & .017 & .130 & .275 & . 170 & .133 & .384 & 1.000 & .225 & . 148 & .560 \\
\hline & $\operatorname{lnd} 12$ & .104 & .002 & .179 & .158 & .213 & .573 & .193 & .310 & .142 & .051 & . 041 & .225 & 1.000 & .099 & .229 \\
\hline & Ind 13 & .381 & .023 & .030 & -.028 & . 123 & .050 & -.041 & .005 & .043 & .127 & .065 & .148 & .099 & 1.000 & .177 \\
\hline & Ind 14 & .283 & -.008 & .048 & -.031 & .181 & .099 & .066 & .271 & .125 & .097 & .339 & .560 & .229 & .177 & 1.000 \\
\hline \multirow[t]{15}{*}{ Sig. (1-tailed) } & Dep. & & .036 & .001 & .001 & .000 & . .171 & .107 & .001 & .168 & .000 & .096 & .010 & .092 & .000 & .000 \\
\hline & Ind 1 & .036 & & .000 & .000 & . 097 & . 177 & .263 & .000 & . 094 & .021 & .337 & .263 & .490 & .383 & .460 \\
\hline & Ind 2 & .001 & .000 & & .000 & .001 & .186 & .328 & .000 & .268 & .037 & . 093 & .263 & . 011 & .349 & .270 \\
\hline & Ind 3 & .001 & .000 & .000 & & .001 & .235 & .193 & .000 & .128 & .086 & .028 & 164 & .021 & .361 & .348 \\
\hline & Ind 4 & .000 & .097 & .001 & .001 & & .105 & .017 & .000 & .048 & .001 & .424 & .254 & .003 & .058 & .010 \\
\hline & Ind 5 & .171 & .177 & .186 & .235 & 105 & & . 002 & .030 & .256 & . 171 & . 173 & .412 & .000 & .261 & .102 \\
\hline & Ind 6 & .107 & .263 & .328 & .193 & .017 & .002 & & .310 & .087 & . 199 & .118 & .047 & .006 & .302 & .201 \\
\hline & Ind 7 & .001 & .000 & .000 & .000 & .000 & .030 & .310 & & .006 & .008 & .391 & .000 & .000 & .475 & .000 \\
\hline & Ind 8 & .168 & .094 & .268 & .128 & .048 & .256 & .087 & .006 & & .001 & .002 & .014 & .034 & .290 & .054 \\
\hline & Ind 9 & .000 & .021 & .037 & .086 & .001 & .171 & .199 & .008 & .001 & & .044 & .044 & .256 & .052 & .106 \\
\hline & Ind 10 & .096 & .337 & .093 & .028 & .424 & .173 & .118 & .391 & .002 & . 044 & & .000 & .302 & .202 & .000 \\
\hline & Ind 11 & .010 & .263 & .263 & .164 & .254 & .412 & .047 & .000 & .014 & .044 & .000 & & .002 & . 029 & .000 \\
\hline & Ind 12 & .092 & .490 & .011 & .021 & .003 & .000 & .006 & .000 & .034 & .256 & .302 & .002 & & .103 & .002 \\
\hline & Ind 13 & .000 & .383 & .349 & .361 & .058 & .261 & .302 & .475 & .290 & .052 & .202 & .029 & 103 & & .011 \\
\hline & Ind 14 & .000 & .460 & .270 & .348 & .010 & .102 & .201 & .000 & .054 & .106 & .000 & .000 & .002 & .011 & \\
\hline \multirow[t]{15}{*}{$\bar{N}$} & Dep. & 166 & 166 & 166 & 166 & 166 & 166 & 166 & 166 & 166 & 166 & 166 & 166 & 166 & 166 & 166 \\
\hline & Ind 1 & 166 & 166 & 166 & 166 & 166 & 166 & 166 & 166 & 166 & 166 & 166 & 166 & 166 & 166 & 166 \\
\hline & Ind 2 & 166 & 166 & 166 & 166 & 166 & 166 & 166 & 166 & 166 & 166 & 166 & 166 & 166 & 166 & 166 \\
\hline & Ind 3 & 166 & $\begin{array}{l}166 \\
\end{array}$ & 166 & 166 & 166 & 166 & 166 & 166 & 166 & 166 & 166 & 166 & 166 & 166 & 166 \\
\hline & Ind 4 & 166 & 166 & 166 & 166 & 166 & 166 & 166 & 166 & 166 & 166 & 166 & 166 & 166 & 166 & 166 \\
\hline & Ind 5 & 166 & 166 & 166 & 166 & 166 & 166 & 166 & 166 & 166 & 166 & 166 & 166 & 166 & 166 & 166 \\
\hline & Ind 6 & 166 & 166 & 166 & 166 & 166 & 166 & 166 & 166 & 166 & 166 & 166 & 166 & 166 & 166 & 166 \\
\hline & Ind 7 & 166 & 166 & 166 & 166 & 166 & 166 & 166 & 166 & 166 & 166 & 166 & 166 & 166 & 166 & 166 \\
\hline & Ind 8 & $\begin{array}{l}100 \\
166\end{array}$ & $\begin{array}{l}100 \\
166\end{array}$ & $\begin{array}{l}100 \\
166\end{array}$ & $\begin{array}{l}100 \\
166\end{array}$ & 166 & $\begin{array}{l}100 \\
166\end{array}$ & $\begin{array}{l}160 \\
166\end{array}$ & 166 & 166 & 166 & 166 & 166 & 166 & 166 & 166 \\
\hline & Ind 9 & 166 & 166 & 166 & 166 & 166 & 166 & 166 & 166 & 166 & 166 & 166 & 166 & 166 & 166 & 166 \\
\hline & Ind 10 & $\begin{array}{l}100 \\
166\end{array}$ & $\begin{array}{l}160 \\
166\end{array}$ & $\begin{array}{l}160 \\
166\end{array}$ & 166 & 166 & $\begin{array}{l}100 \\
166\end{array}$ & 160 & 166 & 166 & 166 & 166 & 166 & 166 & 166 & 166 \\
\hline & Ind 11 & 166 & 166 & 166 & 166 & 166 & 166 & 166 & 166 & 166 & 166 & 166 & 166 & 166 & 166 & 166 \\
\hline & Ind 12 & 166 & 166 & 166 & 166 & 166 & 166 & 166 & 166 & 166 & 166 & 166 & 166 & 166 & 166 & 166 \\
\hline & Ind 13 & 166 & 166 & 166 & 166 & 166 & 166 & 166 & 166 & 166 & 166 & 166 & 166 & 166 & 166 & 166 \\
\hline & Ind 14 & 166 & 166 & 166 & 166 & 166 & 166 & 166 & 166 & 166 & 166 & 166 & 166 & 166 & 166 & 166 \\
\hline
\end{tabular}


Table E.8: Research Question 1: Variables Entered/Removed During Stepwise Regression

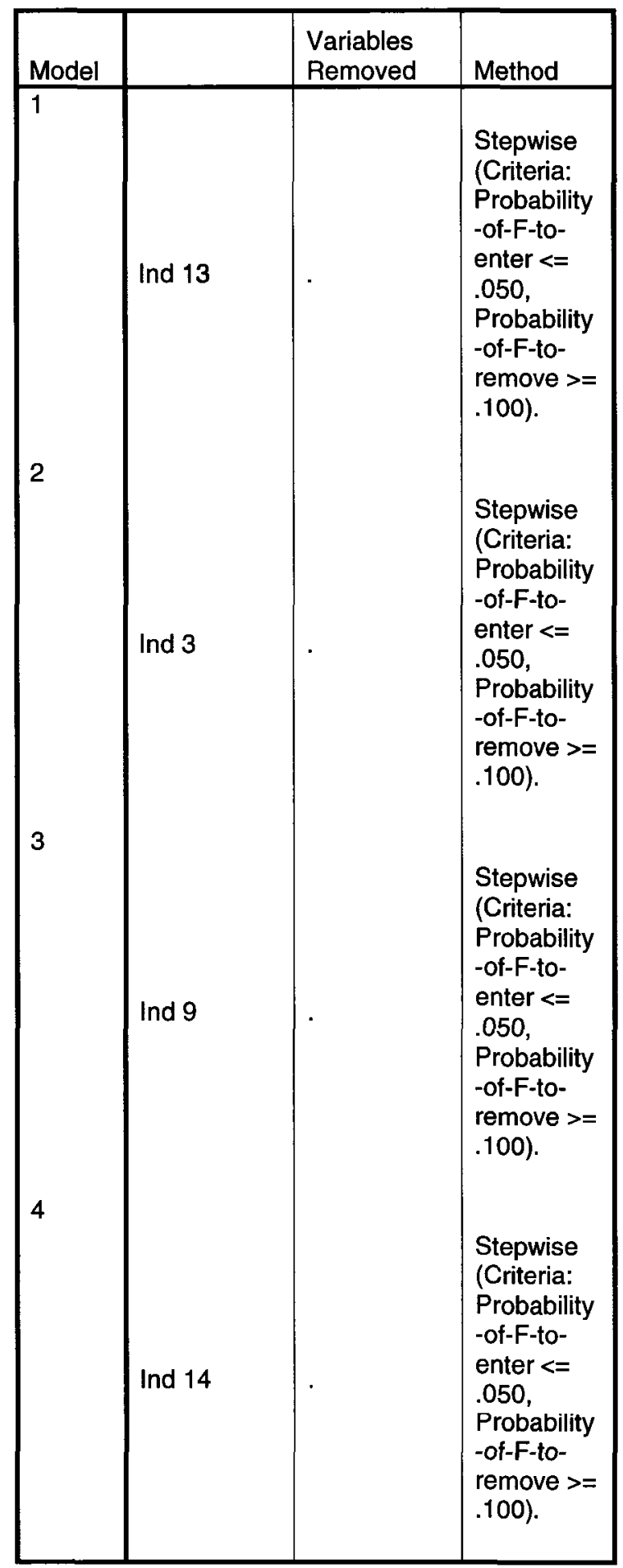

a Dependent Variable: Log dependent 
Table E.9: Research Question 1: Excluded Variables in Stepwise Regression

\begin{tabular}{|c|c|c|c|c|c|c|c|c|}
\hline \multicolumn{9}{|c|}{ Excluded Variables } \\
\hline \multirow{2}{*}{\multicolumn{2}{|c|}{ Model }} & \multirow[b]{2}{*}{ Beta In } & \multirow[b]{2}{*}{$t$} & \multirow[b]{2}{*}{ Sig. } & \multirow[b]{2}{*}{$\begin{array}{c}\text { Partial } \\
\text { Correlation }\end{array}$} & \multicolumn{3}{|c|}{ Collinearity Statistics } \\
\hline & & & & & & Tolerance & VIF & $\begin{array}{l}\text { Minimum } \\
\text { Tolerance }\end{array}$ \\
\hline \multirow[t]{13}{*}{1} & Ind 1 & $.149^{\mathrm{a}}$ & 2.081 & .039 & .161 & .999 & 1.001 & .999 \\
\hline & Ind 2 & $.221^{\mathrm{a}}$ & 3.140 & .002 & .239 & .999 & 1.001 & .999 \\
\hline & Ind 3 & $.264^{\mathrm{a}}$ & 3.801 & .000 & .285 & .999 & 1.001 & .999 \\
\hline & Ind 4 & $.211^{\mathrm{a}}$ & 2.964 & .003 & .226 & .985 & 1.015 & .985 \\
\hline & Ind 5 & $.055^{\mathrm{a}}$ & .762 & .447 & .060 & .997 & 1.003 & .997 \\
\hline & Ind 6 & $.113^{\mathrm{a}}$ & 1.567 & .119 & .122 & .998 & 1.002 & .998 \\
\hline & Ind 7 & $.240^{\mathrm{a}}$ & 3.425 & .001 & .259 & 1.000 & 1.000 & 1.000 \\
\hline & Ind 8 & $.092^{\mathrm{a}}$ & 1.274 & .205 & .099 & .998 & 1.002 & .998 \\
\hline & Ind 9 & $.257^{\mathrm{a}}$ & 3.657 & .000 & .275 & .984 & 1.016 & .984 \\
\hline & Ind 10 & $.077^{\mathrm{a}}$ & 1.069 & .287 & .083 & .996 & 1.004 & .996 \\
\hline & Ind 11 & $.127^{a}$ & 1.747 & .083 & .136 & .978 & 1.022 & .978 \\
\hline & Ind 12 & $.067^{a}$ & .918 & .360 & .072 & .990 & 1.010 & .990 \\
\hline & Ind 14 & $.222^{\mathrm{a}}$ & 3.109 & .002 & .237 & .969 & 1.033 & .969 \\
\hline \multirow[t]{12}{*}{2} & Ind 1 & $.074^{\mathrm{b}}$ & 1.019 & .310 & .080 & .904 & 1.106 & .904 \\
\hline & Ind 2 & $.106^{b}$ & 1.261 & .209 & .099 & .682 & 1.467 & .682 \\
\hline & Ind 4 & $.152^{b}$ & 2.128 & .035 & .165 & .920 & 1.087 & .920 \\
\hline & Ind 5 & $.040^{\mathrm{b}}$ & .573 & .567 & .045 & .994 & 1.006 & .994 \\
\hline & Ind 6 & $.132^{\mathrm{b}}$ & 1.904 & .059 & .148 & .994 & 1.006 & .994 \\
\hline & Ind 7 & $.169^{b}$ & 2.318 & .022 & .179 & .883 & 1.133 & .882 \\
\hline & Ind 8 & $.117^{b}$ & 1.681 & .095 & .131 & .990 & 1.010 & .990 \\
\hline & Ind 9 & $.230^{\mathrm{b}}$ & 3.369 & .001 & .256 & .972 & 1.029 & .972 \\
\hline & Ind 10 & $.119^{b}$ & 1.699 & .091 & .132 & .974 & 1.027 & .974 \\
\hline & Ind 11 & $.147^{b}$ & 2.111 & .036 & .164 & .973 & 1.028 & .973 \\
\hline & Ind 12 & $.024^{b}$ & .345 & .731 & .027 & .964 & 1.037 & .964 \\
\hline & Ind 14 & $.229^{\mathrm{b}}$ & 3.352 & .001 & .255 & .968 & 1.033 & .968 \\
\hline \multirow[t]{11}{*}{3} & Ind 1 & $.043^{\mathrm{c}}$ & .599 & .550 & .047 & .888 & 1.127 & .888 \\
\hline & Ind 2 & $.082^{c}$ & 1.000 & .319 & .079 & .676 & 1.479 & .676 \\
\hline & Ind 4 & $.106^{\mathrm{c}}$ & 1.477 & .142 & .116 & .876 & 1.142 & .876 \\
\hline & Ind 5 & $.026^{\mathrm{c}}$ & .382 & .703 & .030 & .990 & 1.010 & .968 \\
\hline & Ind 6 & $.114^{\mathrm{c}}$ & 1.693 & .092 & .132 & .987 & 1.013 & .966 \\
\hline & Ind 7 & $.134^{c}$ & 1.859 & .065 & .145 & .860 & 1.163 & .860 \\
\hline & Ind 8 & $.062^{c}$ & .881 & .379 & .069 & .924 & 1.083 & .907 \\
\hline & Ind 10 & $.088^{\mathrm{C}}$ & 1.272 & .205 & .100 & .954 & 1.048 & .952 \\
\hline & Ind 11 & $.120^{\circ}$ & 1.756 & .081 & .137 & .958 & 1.044 & .956 \\
\hline & Ind 12 & $.019^{\mathrm{c}}$ & .282 & .778 & .022 & .964 & 1.037 & .962 \\
\hline & Ind 14 & $.212^{\mathrm{c}}$ & 3.179 & .002 & .243 & .962 & 1.040 & .955 \\
\hline \multirow[t]{10}{*}{4} & Ind 1 & $.044^{d}$ & .636 & .526 & .050 & .888 & 1.127 & .888 \\
\hline & Ind 2 & $.066^{\mathrm{d}}$ & .827 & .409 & .065 & .673 & 1.485 & .673 \\
\hline & Ind 4 & $.071^{\mathrm{d}}$ & 1.007 & .315 & .079 & .853 & 1.173 & .853 \\
\hline & Ind 5 & $.007^{\mathrm{d}}$ & .111 & .912 & .009 & .982 & 1.018 & .954 \\
\hline & Ind 6 & $.101^{\mathrm{d}}$ & 1.529 & .128 & .120 & .983 & 1.017 & .951 \\
\hline & Ind 7 & $.074^{\mathrm{d}}$ & 1.007 & .315 & .079 & .786 & 1.272 & .786 \\
\hline & Ind 8 & $.037^{d}$ & .538 & .591 & .043 & .911 & 1.097 & .905 \\
\hline & Ind 10 & $.020^{d}$ & .283 & .778 & .022 & .852 & 1.173 & .852 \\
\hline & Ind 11 & $.006^{\mathrm{d}}$ & .077 & .939 & .006 & .675 & 1.482 & .675 \\
\hline & Ind 12 & $-.029^{d}$ & -.423 & .673 & -.033 & .917 & 1.091 & .914 \\
\hline \multicolumn{9}{|c|}{ a. Predictors in the Model: (Constant), Ind 13} \\
\hline \multicolumn{9}{|c|}{ b. Predictors in the Model: (Constant), Ind 13 , Ind 3} \\
\hline \multicolumn{9}{|c|}{ c. Predictors in the Model: (Constant), Ind 13, Ind 3 , Ind 9} \\
\hline \multicolumn{9}{|c|}{ d. Predictors in the Model: (Constant), Ind 13, Ind 3, Ind 9, Ind 14} \\
\hline & pendent & ariable: $\log$ & Jendent & & & & & \\
\hline
\end{tabular}




\section{E.2. Research Question 1: Individual Dependent Variables Measuring Success}

In this Section, we report the stepwise multiple regression results taking each of the five variables measuring Success, viz., Reduced Delivery Schedules (D1), Increased ROI (D2), Increased Ability to Meet with Current Customer Requirements (D3), Increased Flexibility to Meet with Changing Customer Requirements (D4), and Improved Business Processes (D5), as the dependent variables. The procedure taken is similar to that used in the Chapter 6 for the regression analysis of Success. Where the log transformation of the dependent variable gives a better regression result, the dependent variable has been log transformed in such a case. If analysis revealed that that was not the case, they were left untransformed. In the regression results below, only the most important tables are shown.

\section{E.2.1. Reduced Delivery Schedules}

Table E.10: Research Question 1: Regression Model Summary for Reduced Delivery Schedules

\begin{tabular}{l|r|r|r|r|}
\hline Model & $\mathrm{R}$ & R Square & $\begin{array}{c}\text { Adjusted R } \\
\text { Square }\end{array}$ & $\begin{array}{r}\text { Std. Error of } \\
\text { the Estimate }\end{array}$ \\
\hline 1 & $.286(\mathrm{a})$ & .082 & .076 & .928 \\
2 & $.327(\mathrm{~b})$ & .107 & .096 & .918 \\
\hline
\end{tabular}
a Predictors: (Constant), Ind 13
b Predictors: (Constant), Ind 13, Ind 14
c Dependent Variable: Dep 1


Table E.11: Research Question 1: ANOVA Table from Regression Analysis with Reduced Delivery Schedules

\begin{tabular}{|ll|r|r|r|r|r|}
\hline \multicolumn{1}{|c|}{ ANOVAc } \\
Model & & $\begin{array}{c}\text { Sum of } \\
\text { Squares }\end{array}$ & df & Mean Square & \multicolumn{1}{c|}{$\mathrm{F}$} & Sig. \\
\hline 1 & Regression & 12.573 & 1 & 12.573 & 14.587 & $.000^{\mathrm{a}}$ \\
& Residual & 141.355 & 164 & .862 & & \\
& Total & 153.928 & 165 & & & $.000^{\mathrm{b}}$ \\
& Regression & 16.440 & 2 & 8.220 & 9.746 & \\
& Residual & 137.487 & 163 & .843 & & \\
& Total & 153.928 & 165 & & & \\
\end{tabular}
a. Predictors: (Constant), Ind 13
b. Predictors: (Constant), Ind 13, Ind 14
c. Dependent Variable: Dep 1

Table E.12: Research Question 1: Regression Coefficients for Reduced Delivery Schedules

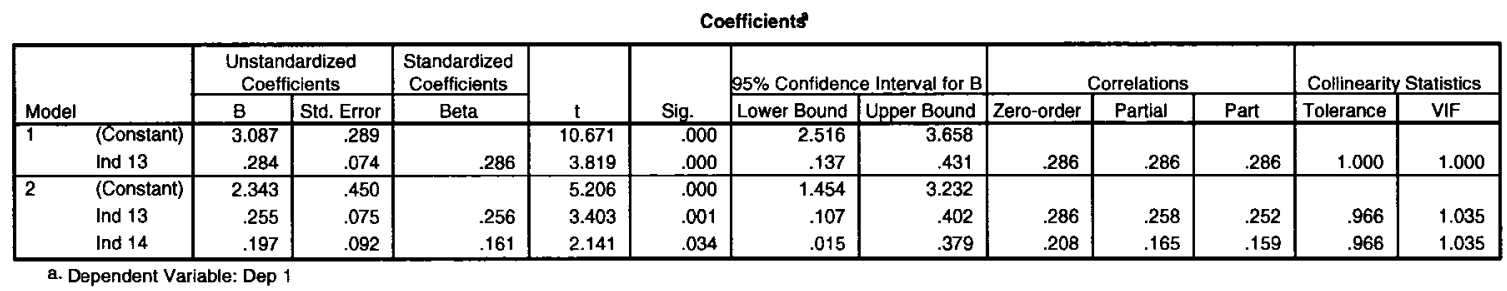

On the basis of the above regression results, it can be inferred that the best regression model for D1 that can be had with the obtained data is:

$$
\text { D1 }=2.343+.255(\text { Ind13 })+.197(\text { Ind14 })
$$




\section{E.2.2. Increased ROI}

Table E.13: Research Question 1: Regression Model Summary for Increased ROI

\begin{tabular}{|c|c|c|c|c|}
\hline Model & $\mathbf{R}$ & R Square & $\begin{array}{c}\text { Adjusted R } \\
\text { Square }\end{array}$ & $\begin{array}{l}\text { Std. Error of } \\
\text { the Estimate }\end{array}$ \\
\hline 1 & $.370(a)$ & .137 & .131 & .22838 \\
\hline 2 & $.483(b)$ & .233 & .223 & .21593 \\
\hline 3 & $.520(\mathrm{c})$ & .271 & .257 & .21124 \\
\hline
\end{tabular}

Table E.14: Research Question 1: ANOVA Table from Regression Analysis with Increased ROI

ANOVA

\begin{tabular}{|ll|r|r|r|r|r|}
\hline Model & & $\begin{array}{c}\text { Sum of } \\
\text { Squares }\end{array}$ & df & Mean Square & \multicolumn{1}{c|}{ F } & Sig. \\
\hline 1 & Regression & 1.322 & 1 & 1.322 & 25.351 & $.000^{\mathrm{a}}$ \\
& Residual & 8.345 & 160 & .052 & & \\
& Total & 9.667 & 161 & & & \\
\hline 2 & Regression & 2.254 & 2 & 1.127 & 24.166 & $.000^{\mathrm{b}}$ \\
& Residual & 7.414 & 159 & .047 & & \\
& Total & 9.667 & 161 & & & $.000^{\mathrm{c}}$ \\
\hline 3 & Regression & 2.617 & 3 & .872 & 19.546 & \\
& Residual & 7.051 & 158 & .045 & & \\
& Total & 9.667 & 161 & & & \\
\end{tabular}
a. Predictors: (Constant), Ind 14
b. Predictors: (Constant), Ind 14, Ind 9
c. Predictors: (Constant), Ind 14, Ind 9, Ind 2
d. Dependent Variable: LOGD2 
Table E.15: Research Question 1: Regression Coefficients for Increased ROI

\begin{tabular}{|c|c|c|c|c|c|c|c|c|c|c|c|c|c|}
\hline \multicolumn{14}{|c|}{ Coefficients" } \\
\hline & & \multicolumn{2}{|c|}{$\begin{array}{l}\text { Unstandardized } \\
\text { Coefficients }\end{array}$} & \multirow{2}{*}{$\begin{array}{c}\begin{array}{c}\text { Standardized } \\
\text { Coefficients }\end{array} \\
\text { Beta }\end{array}$} & \multirow[b]{2}{*}{1} & \multirow[b]{2}{*}{ Sig. } & \multicolumn{2}{|c|}{$95 \%$ Contidence Interval for $\mathrm{B}$} & \multicolumn{3}{|c|}{ Correlations } & \multicolumn{2}{|c|}{ Collinearity Statistics } \\
\hline & Madel & B & Sid Error & & & & Lower Bound & Upper Bound & Zero-order & Partial & Part & Tolerance & VIF \\
\hline & (Constant) & .946 & .100 & & 9.496 & .000 & .749 & 1.143 & & & & & \\
\hline \multirow[t]{3}{*}{2} & (Constant) & .814 & .099 & & 8.251 & .000 & .620 & 1.009 & & & & & \\
\hline & Ind 14 & .100 & .022 & 324 & 4.611 & .000 & .057 & .143 & .370 & .343 & .320 & .978 & 1.022 \\
\hline & Ind 9 & .053 & .012 & .314 & 4.469 & .000 & .030 & .076 & .361 & .334 & .310 & .978 & 1.022 \\
\hline \multirow[t]{2}{*}{3} & (Constant) & .626 & .117 & & 5.341 & .000 & .394 & .857 & & & & & \\
\hline & Ind 2 & .052 & .018 & .196 & 2.852 & .005 & .016 & .088 & .258 & .221 & .194 & .974 & 1.026 \\
\hline
\end{tabular}

a. Dependent Variable: LOGD2

On the basis of the above regression results, it can be inferred that the best regression model is:

$\log ($ D2 $)=.626+.098($ Ind14 $)+.048($ Ind9 $)+.052($ Ind2 $)$

E.2.3. Increased Ability to Meet with Current Customer Requirements

Table E.16: Research Question 1: Regression Model Summary for Increased Ability to Meet with Current Customer Requirements

\begin{tabular}{|l|l|r|r|r|}
\hline Model & R & R Square & $\begin{array}{c}\text { Adjusted R } \\
\text { Square }\end{array}$ & $\begin{array}{c}\text { Std. Error of } \\
\text { the Estimate }\end{array}$ \\
\hline 1 & $.334(\mathrm{a})$ & .112 & .106 & .13884 \\
2 & $.387(\mathrm{~b})$ & .150 & .139 & .13627 \\
\hline
\end{tabular}

a Predictors: (Constant), Ind 3

b Predictors: (Constant), Ind 3 , Ind 7

c Dependent Variable: LOGD3 
Table E.17: Research Question 1: ANOVA Table from Regression Analysis with Increased Ability to Meet with Current Customer Requirements

ANOVAC

\begin{tabular}{|ll|r|r|r|r|r|}
\hline \multicolumn{1}{|c|}{ Model } & $\begin{array}{c}\text { Sum of } \\
\text { Squares }\end{array}$ & df & Mean Square & \multicolumn{1}{c|}{ F } & Sig. \\
\hline 1 & Regression & .398 & 1 & .398 & 20.657 & $.000^{\mathrm{a}}$ \\
& Residual & 3.161 & 164 & .019 & & \\
& Total & 3.559 & 165 & & & \\
\hline 2 & Regression & .533 & 2 & .266 & 14.340 & $.000^{\mathrm{b}}$ \\
& Residual & 3.027 & 163 & .019 & & \\
& Total & 3.559 & 165 & & & \\
\hline
\end{tabular}

a. Predictors: (Constant), Ind 3

b. Predictors: (Constant), Ind 3, Ind 7

c. Dependent Variable: LOGD3

Table E.18: Research Question 1: Regression Coefficients Table for Increased Ability to Meet with Current Customer Requirements

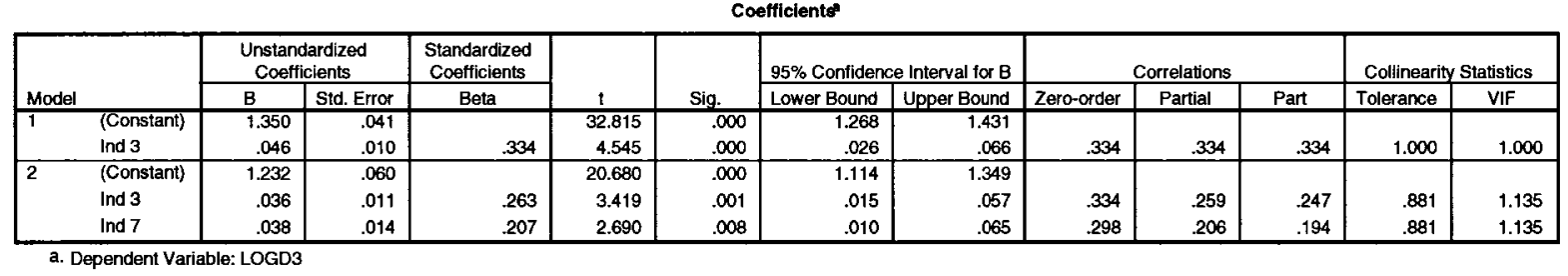

On the basis of the above regression results, it can be inferred that the best regression model is:

$$
\log (\mathrm{D3})=1.232+.036(\operatorname{Ind} 3)+.038(\operatorname{Ind} 7)
$$




\section{E.2.4. Increased Flexibility to Meet with Changing Customer Requirements}

Table E.19: Research Question 1: Regression Model Summary for Increased Flexibility to Meet with Changing Customer Requirements

\begin{tabular}{|l|l|r|r|r|}
\hline Model & R & R Square & $\begin{array}{c}\text { Adjusted R } \\
\text { Square }\end{array}$ & $\begin{array}{c}\text { Std. Error of } \\
\text { the Estimate }\end{array}$ \\
\hline 1 & $.262(\mathrm{a})$ & .069 & .063 & .516 \\
\hline
\end{tabular}

a Predictors: (Constant), Ind 3

b Dependent Variable: Dep 4

Table E.20: Research Question : ANOVA Table from Regression Analysis with Increased Flexibility to Meet with Changing Customer Requirements

\section{ANOVA}

\begin{tabular}{|ll|r|r|r|r|r|}
\hline \multicolumn{1}{|c|}{} & \multicolumn{1}{|c|}{$\begin{array}{c}\text { Sum of } \\
\text { Model }\end{array}$} & Squares & $\mathrm{df}$ & Mean Square & \multicolumn{1}{c|}{$\mathrm{F}$} & \multicolumn{1}{c|}{ Sig. } \\
\hline 1 & Regression & 3.222 & 1 & 3.222 & 12.107 & $.001^{\mathrm{a}}$ \\
& Residual & 43.651 & 164 & .266 & & \\
& Total & 46.873 & 165 & & & \\
\hline
\end{tabular}

a. Predictors: (Constant), Ind 3

b. Dependent Variable: Dep 4

Table E.21: Research Question 1: Regression Coefficients for Increased Flexibility to Meet with Changing Customer Requirements

\begin{tabular}{|c|c|c|c|c|c|c|c|c|c|c|c|c|}
\hline \multicolumn{13}{|c|}{ Coefficients } \\
\hline \multirow[b]{2}{*}{ Model } & \multicolumn{2}{|c|}{$\begin{array}{l}\text { Unstandardized } \\
\text { Coefficients } \\
\end{array}$} & \multirow{2}{*}{$\begin{array}{c}\text { Standardized } \\
\text { Coefficients } \\
\text { Beta }\end{array}$} & \multirow[b]{2}{*}{$t$} & \multirow[b]{2}{*}{ sig. } & \multicolumn{2}{|c|}{$95 \%$ Confidence Interval for B } & \multicolumn{3}{|c|}{ Correlations } & \multicolumn{2}{|c|}{ Collinearity Statistics } \\
\hline & B & Std. Error & & & & Lower Bound & Upper Bound & Zero-order & Partial & Part & Tolerance & VIF \\
\hline $\begin{array}{ll}1 & \text { (Constant) } \\
\text { ind } 3\end{array}$ & $\begin{array}{r}4.240 \\
.130\end{array}$ & $\begin{array}{l}.153 \\
.037\end{array}$ & .262 & $\begin{array}{r}27.743 \\
3.479\end{array}$ & $\begin{array}{l}.000 \\
.001\end{array}$ & $\begin{array}{r}.938 \\
.056\end{array}$ & $\begin{array}{r}.542 \\
.204\end{array}$ & .262 & .262 & .262 & 1.000 & 1.000 \\
\hline
\end{tabular}

Although the above regression results here show a poor model, on the basis of the above results, it can be inferred that the best regression model fitting the data is:

$$
\text { D4 }=4.24+.013(\text { Ind3) }
$$




\section{E.2.5. Improved Business Processes}

Table E.22: Research Question 1: Regression Model Summary for Improved Business Processes

\begin{tabular}{|c|c|c|c|c|}
\hline Model & $\mathrm{R}$ & R Square & $\begin{array}{l}\text { Adjusted R } \\
\text { Square }\end{array}$ & $\begin{array}{l}\text { Std. Error of } \\
\text { the Estimate }\end{array}$ \\
\hline 1 & $.422(a)$ & .178 & .173 & .20054 \\
\hline 2 & $.473(\mathrm{~b})$ & .224 & .214 & .19553 \\
\hline 3 & $.496(c)$ & .246 & .232 & .19327 \\
\hline
\end{tabular}

Table E.23: Research Question 1: ANOVA Table from Regression Analysis with Improved Business Processes

ANOVA

\begin{tabular}{|ll|r|r|r|r|r|}
\hline \multicolumn{1}{|c|}{ Model } & \multicolumn{1}{c|}{$\begin{array}{c}\text { Sum of } \\
\text { Squares }\end{array}$} & df & Mean Square & \multicolumn{1}{c|}{$\mathrm{F}$} & Sig. \\
\hline 1 & Regression & 1.385 & 1 & 1.385 & 34.445 & $.000^{\mathrm{a}}$ \\
& Residual & 6.394 & 159 & .040 & & \\
& Total & 7.780 & 160 & & & \\
\hline 2 & Regression & 1.739 & 2 & .869 & 22.741 & $.000^{\mathrm{b}}$ \\
& Residual & 6.041 & 158 & .038 & & \\
& Total & 7.780 & 160 & & & $.000^{\mathrm{c}}$ \\
\hline 3 & Regression & 1.915 & 3 & .638 & 17.094 & \\
& Residual & 5.864 & 157 & .037 & & \\
& Total & 7.780 & 160 & & & \\
\end{tabular}
a. Predictors: (Constant), Ind 4
b. Predictors: (Constant), Ind 4, Ind 7
c. Predictors: (Constant), Ind 4, Ind 7, Ind 3
d. Dependent Variable: LOGD5 
Table E.24: Research Question 1: Regression Coefficients Table for Improved Business Processes

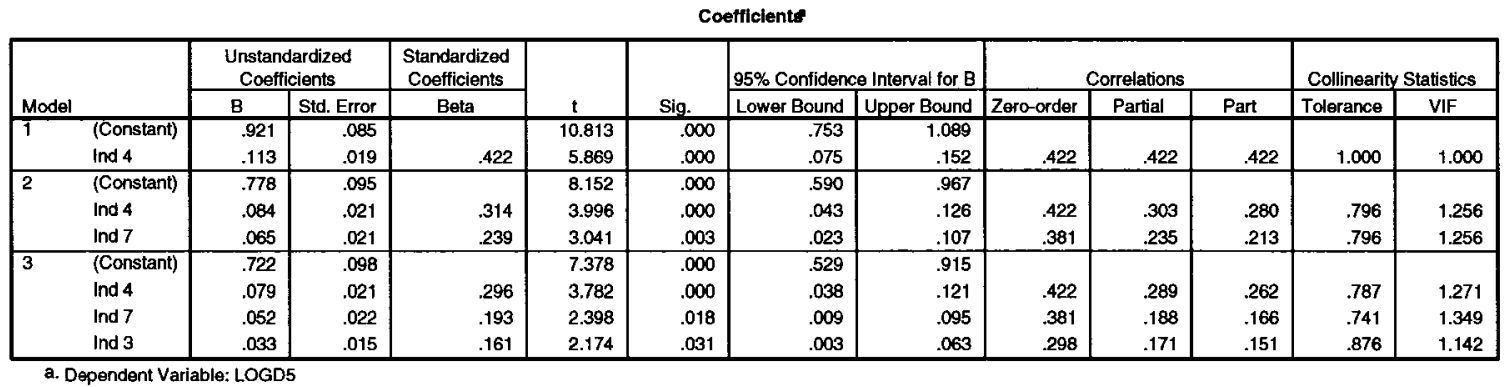

On the basis of the above results, it can be inferred that the best regression model fitting the data is:

$$
\log (\mathrm{D5})=.722+.079(\text { Ind4 })+.052(\text { Ind7 })+.033(\text { Ind3 })
$$

\section{E.3. Research Question 2}

The detailed statistical analysis results obtained while investigating the data corresponding to Research Question 2 are presented in this Section.

\section{E.3.1. Detailed Results of Descriptive Statistics}

In Table E.24, we present the descriptive statistical results for C1, C2, C3, and C4.

Table E.25: Research Question 2: Detailed Descriptive Statistics (Part I)

\begin{tabular}{|lll|r|r|}
\hline & & \multicolumn{1}{|c|}{ Statistic } & Std. Error \\
\hline C1 & Mean & 4.1927 & .06986 \\
& $95 \%$ Confidence & Lower Bound & 4.0548 & \\
Interval for Mean & Upper Bound & & \\
& & 4.3307 & \\
5\% Trimmed Mean & & 4.3155 & \\
Median & 4.4000 & \\
Variance & .805 & \\
Std. Deviation & & .89739 & \\
& & &
\end{tabular}




\begin{tabular}{|c|c|c|c|c|}
\hline & Minimum & & .00 & \\
\hline & Maximum & & 5.00 & \\
\hline & Range & & 5.00 & \\
\hline & Interquartile Range & & .80 & \\
\hline & Skewness & & -2.866 & .189 \\
\hline & Kurtosis & & 10.291 & .376 \\
\hline $\mathrm{C2}$ & Mean & & 4.3455 & .07887 \\
\hline & $\begin{array}{l}95 \% \text { Confidence } \\
\text { Interval for Mean }\end{array}$ & Lower Bound & 4.1897 & \\
\hline & & Upper Bound & & \\
\hline & $5 \%$ Trimmed Mean & & 4.5051 & \\
\hline & Median & & 4.5000 & \\
\hline & Variance & & 1.026 & \\
\hline & Std. Deviation & & 1.01305 & \\
\hline & Minimum & & .00 & \\
\hline & Maximum & & 5.00 & \\
\hline & Range & & 5.00 & \\
\hline & Interquartile Range & & 1.00 & \\
\hline & Skewness & & -2.732 & .189 \\
\hline & Kurtosis & & 8.590 & .376 \\
\hline C3 & Mean & & 3.8606 & .09175 \\
\hline & $\begin{array}{l}95 \% \text { Confidence } \\
\text { Interval for Mean }\end{array}$ & Lower Bound & 3.6794 & \\
\hline & & Upper Bound & & \\
\hline & $5 \%$ Trimmed Mean & & 4.0101 & \\
\hline & Median & & 4.0000 & \\
\hline & Variance & & 1.389 & \\
\hline & Std. Deviation & & 1.17855 & \\
\hline & Minimum & & .00 & \\
\hline & Maximum & & 5.00 & \\
\hline & Range & & 5.00 & \\
\hline & Interquartile Range & & 1.00 & \\
\hline & Skewness & & -1.807 & .189 \\
\hline & Kurtosis & & 3.583 & .376 \\
\hline $\mathrm{C} 4$ & Mean & & 4.1919 & .07247 \\
\hline & $\begin{array}{l}95 \% \text { Confidence } \\
\text { Interval for Mean }\end{array}$ & & 4.0488 & \\
\hline & & Upper Bound & 4.3350 & \\
\hline & $5 \%$ Trimmed Mean & & 4.3155 & \\
\hline & Median & & 4.5000 & \\
\hline & Variance & & .867 & \\
\hline & Std. Deviation & & .93090 & \\
\hline & Minimum & & .00 & \\
\hline & Maximum & & 5.00 & \\
\hline
\end{tabular}

261 


\begin{tabular}{|l|r|r|} 
Range & 5.00 & \\
Interquartile Range & .75 & \\
Skewness & -2.373 & .189 \\
Kurtosis & 6.999 & .376 \\
\hline
\end{tabular}

In Table E.25, we present the detailed descriptive statistical analysis results for each of the change items constituting to form the change items $\mathrm{C} 1, \mathrm{C} 2, \mathrm{C} 3$, and $\mathrm{C} 4$ (such as C1.1, C1.2, C1.3, etc.).

Table E.26: Research Question 2: Detailed Descriptive Statistics (Part II)

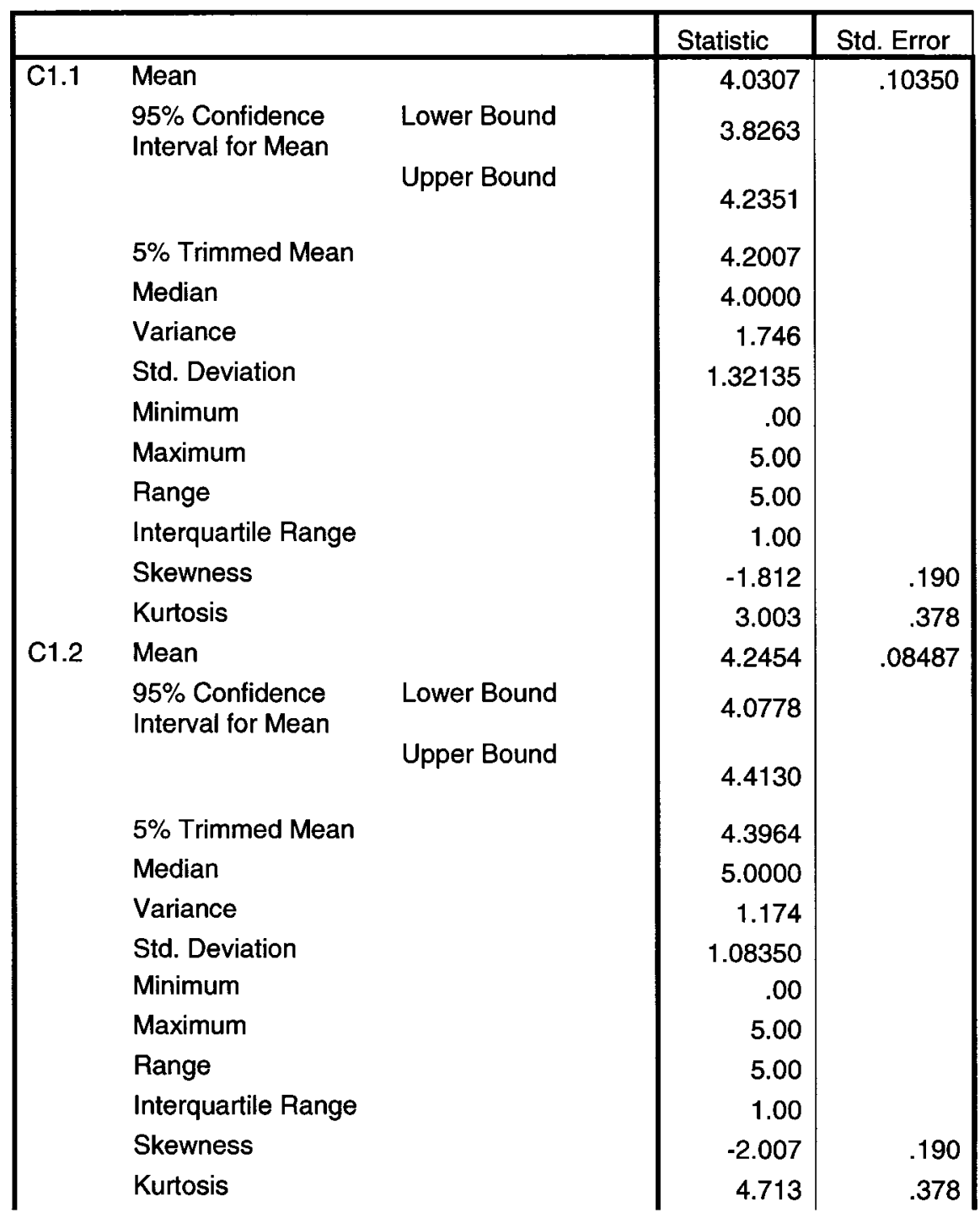




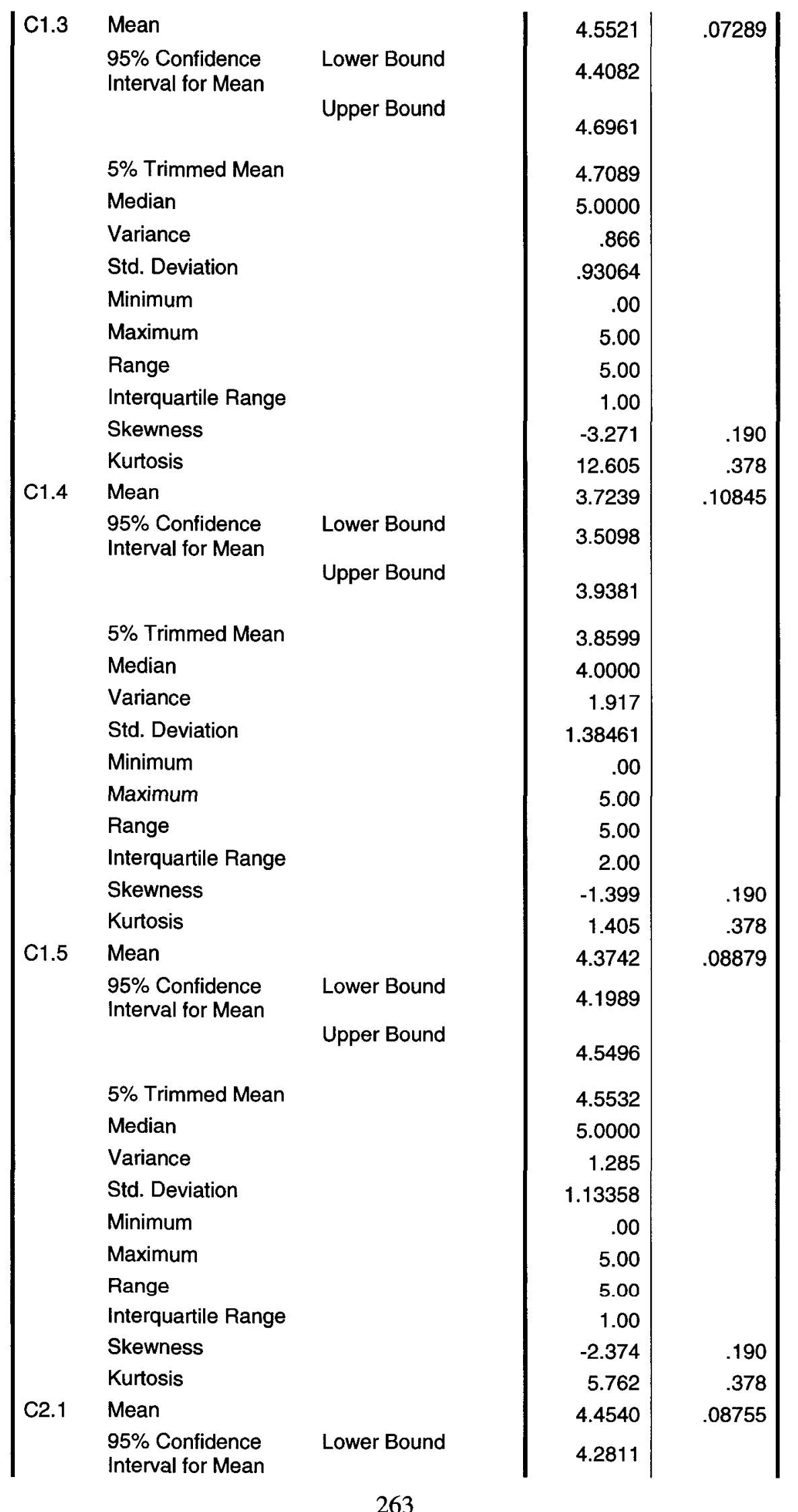




\begin{tabular}{|c|c|c|c|c|}
\hline & & Upper Bound & 4.6269 & \\
\hline & $5 \%$ Trimmed Mean & & 4.6554 & \\
\hline & Median & & 5.0000 & \\
\hline & Variance & & 1.249 & \\
\hline & Std. Deviation & & 1.11777 & \\
\hline & Minimum & & .00 & \\
\hline & Maximum & & 5.00 & \\
\hline & Range & & 5.00 & \\
\hline & Interquartile Range & & 1.00 & \\
\hline & Skewness & & -2.904 & .190 \\
\hline & Kurtosis & & 8.685 & .378 \\
\hline $\mathrm{C} 2.2$ & Mean & & 4.2209 & .08704 \\
\hline & $\begin{array}{l}95 \% \text { Confidence } \\
\text { Interval for Mean }\end{array}$ & Lower Bound & 4.0490 & \\
\hline & & Upper Bound & 4.3927 & \\
\hline & $5 \%$ Trimmed Mean & & 4.3828 & \\
\hline & Median & & 4.0000 & \\
\hline & Variance & & 1.235 & \\
\hline & Std. Deviation & & 1.11125 & \\
\hline & Minimum & & .00 & \\
\hline & Maximum & & 5.00 & \\
\hline & Range & & 5.00 & \\
\hline & Interquartile Range & & 1.00 & \\
\hline & Skewness & & -2.224 & .190 \\
\hline & Kurtosis & & 5.683 & .378 \\
\hline C4.1 & Mean & & 4.6012 & .08049 \\
\hline & $\begin{array}{l}95 \% \text { Confidence } \\
\text { Interval for Mean }\end{array}$ & Lower Bound & 4.4423 & \\
\hline & & Upper Bound & 4.7602 & \\
\hline & $5 \%$ Trimmed Mean & & 4.7975 & \\
\hline & Median & & 5.0000 & \\
\hline & Variance & & 1.056 & \\
\hline & Std. Deviation & & 1.02764 & \\
\hline & Minimum & & .00 & \\
\hline & Maximum & & 5.00 & \\
\hline & Range & & 5.00 & \\
\hline & Interquartile Range & & .00 & \\
\hline & Skewness & & -3.352 & .190 \\
\hline & Kurtosis & & 11.495 & .378 \\
\hline C4.2 & Mean & & 3.6319 & .10622 \\
\hline & $\begin{array}{l}95 \% \text { Confidence } \\
\text { Interval for Mean }\end{array}$ & Lower Bound & 3.4222 & \\
\hline & & Upper Bound & 3.8417 & \\
\hline
\end{tabular}




\begin{tabular}{|c|c|c|c|c|}
\hline & $5 \%$ Trimmed Mean & & 3.7577 & \\
\hline & Median & & 4.0000 & \\
\hline & Variance & & 1.839 & \\
\hline & Std. Deviation & & 1.35609 & \\
\hline & Minimum & & .00 & \\
\hline & Maximum & & 5.00 & \\
\hline & Range & & 5.00 & \\
\hline & Interquartile Range & & 2.00 & \\
\hline & Skewness & & -1.305 & .190 \\
\hline & Kurtosis & & 1.201 & .378 \\
\hline C4.3 & Mean & & 4.1166 & .10312 \\
\hline & $\begin{array}{l}95 \% \text { Confidence } \\
\text { Interval for Mean }\end{array}$ & Lower Bound & 3.9129 & \\
\hline & & Upper Bound & 4.3202 & \\
\hline & $5 \%$ Trimmed Mean & & 4.2962 & \\
\hline & Median & & 5.0000 & \\
\hline & Variance & & 1.733 & \\
\hline & Std. Deviation & & 1.31653 & \\
\hline & Minimum & & .00 & \\
\hline & Maximum & & 5.00 & \\
\hline & Range & & 5.00 & \\
\hline & Interquartile Range & & 1.00 & \\
\hline & Skewness & & -1.976 & .190 \\
\hline & Kurtosis & & 3.512 & .378 \\
\hline C4.4 & Mean & & 4.3865 & .08225 \\
\hline & $\begin{array}{l}95 \% \text { Confidence } \\
\text { Interval for Mean }\end{array}$ & & 4.2241 & \\
\hline & & Upper Bound & 4.5489 & \\
\hline & $5 \%$ Trimmed Mean & & 4.5521 & \\
\hline & Median & & 5.0000 & \\
\hline & Variance & & 1.103 & \\
\hline & Std. Deviation & & 1.05013 & \\
\hline & Minimum & & .00 & \\
\hline & Maximum & & 5.00 & \\
\hline & Range & & 5.00 & \\
\hline & Interquartile Range & & 1.00 & \\
\hline & Skewness & & -2.545 & .190 \\
\hline & Kurtosis & & 7.501 & .378 \\
\hline
\end{tabular}




\section{E.3.2. Detailed results of t-test}

Table E.27: Research Question 2: One-Sample Test for Items in C1

\begin{tabular}{|c|c|c|c|c|c|c|}
\hline & \multicolumn{6}{|c|}{ Test Value $=3$} \\
\hline & \multirow[b]{2}{*}{$t$} & \multirow[b]{2}{*}{ df } & \multirow[b]{2}{*}{ Sig. (2-tailed) } & \multirow{2}{*}{$\begin{array}{c}\text { Mean } \\
\text { Difference }\end{array}$} & \multicolumn{2}{|c|}{$\begin{array}{l}95 \% \text { Confidence Interval } \\
\text { of the Difference }\end{array}$} \\
\hline & & & & & Lower & Upper \\
\hline C1.1 & 10.120 & 164 & .000 & 1.03636 & .8342 & 1.2386 \\
\hline C1.2 & 14.868 & 164 & .000 & 1.24848 & 1.0827 & 1.4143 \\
\hline C1.3 & 21.600 & 164 & .000 & 1.55758 & 1.4152 & 1.7000 \\
\hline C1.4 & 6.866 & 164 & .000 & .73939 & .5268 & .9520 \\
\hline C1.5 & 15.725 & 164 & .000 & 1.38182 & 1.2083 & 1.5553 \\
\hline
\end{tabular}

Table E.28: Research Question 2: One-Sample Test for Items in C2

\begin{tabular}{|c|c|r|r|r|r|r|}
\hline & \multicolumn{9}{|c|}{ Test Value = 3 } \\
\cline { 2 - 7 } & & & & & \multicolumn{1}{|c|}{$\begin{array}{c}\text { 95\% Confidence Interval } \\
\text { of the Difference }\end{array}$} \\
\cline { 5 - 8 } & $\mathrm{t}$ & $\mathrm{df}$ & Sig. (2-tailed) & $\begin{array}{c}\text { Mean } \\
\text { Difference }\end{array}$ & Lower & Upper \\
\hline C2.1 & 16.864 & 164 & .000 & 1.46061 & 1.2896 & 1.6316 \\
C2.2 & 14.146 & 163 & .000 & 1.22561 & 1.0545 & 1.3967 \\
\hline
\end{tabular}

Table E.29: Research Question 2: One-Sample Test for Items in C4

\begin{tabular}{|c|c|c|c|c|c|c|}
\hline & \multicolumn{6}{|c|}{ Test Value $=3$} \\
\hline & \multirow[b]{2}{*}{$t$} & \multirow[b]{2}{*}{$d f$} & \multirow[b]{2}{*}{ Sig. (2-tailed) } & \multirow[b]{2}{*}{$\begin{array}{c}\text { Mean } \\
\text { Difference }\end{array}$} & \multicolumn{2}{|c|}{$\begin{array}{l}95 \% \text { Confidence Interval } \\
\text { of the Difference }\end{array}$} \\
\hline & & & & & Lower & Upper \\
\hline C4.1 & 20.180 & 164 & .000 & 1.60606 & 1.4489 & 1.7632 \\
\hline C4.2 & 6.046 & 163 & .000 & .64024 & .4311 & .8493 \\
\hline C4.3 & 10.991 & 164 & .000 & 1.12121 & .9198 & 1.3226 \\
\hline C4.4 & 17.120 & 164 & .000 & 1.39394 & 1.2332 & 1.5547 \\
\hline
\end{tabular}


Table E.30: Research Question 2: Paired Samples Test with Consolidated Change Items

Paired Samples Test

\begin{tabular}{|c|c|c|c|c|c|c|c|c|c|}
\hline & & \multicolumn{5}{|c|}{ Paired Differences } & \multirow[b]{3}{*}{$\mathrm{t}$} & \multirow[b]{3}{*}{ df } & \multirow[b]{3}{*}{ Sig. (2-tailed) } \\
\hline & & \multirow[b]{2}{*}{ Mean } & \multirow[b]{2}{*}{ Std. Deviation } & \multirow{2}{*}{$\begin{array}{l}\text { Std. Error } \\
\text { Mean }\end{array}$} & \multicolumn{2}{|c|}{$\begin{array}{l}95 \% \text { Confidence } \\
\text { Interval of the } \\
\text { Difference }\end{array}$} & & & \\
\hline & & & & & Lower & Upper & & & \\
\hline Pair 1 & $\mathrm{C} 1-\mathrm{C}_{2}$ & -.15273 & .79385 & .06180 & -.27476 & -.03070 & -2.471 & 164 & .014 \\
\hline Pair 2 & $\mathrm{C} 1-\mathrm{C4}$ & .00081 & .71792 & .05589 & -.10955 & .11117 & .014 & 164 & .988 \\
\hline Pair 3 & $\mathrm{C} 3-\mathrm{C}_{4}$ & -.33131 & 1.06663 & .08304 & -.49527 & -.16735 & -3.990 & 164 & .000 \\
\hline
\end{tabular}

Table E.31: Research Question 2: Paired Samples Test with Individual Change Items

Paired Samples Test

\begin{tabular}{|c|c|c|c|c|c|c|c|c|c|}
\hline & & \multicolumn{5}{|c|}{ Paired Differences } & \multirow[b]{3}{*}{$t$} & \multirow[b]{3}{*}{ df } & \multirow[b]{3}{*}{ Sig. (2-tailed) } \\
\hline & & \multirow[b]{2}{*}{ Mean } & \multirow[b]{2}{*}{ Std. Deviation } & \multirow{2}{*}{$\begin{array}{l}\text { Std. Error } \\
\text { Mean }\end{array}$} & \multicolumn{2}{|c|}{$\begin{array}{l}95 \% \text { Confidence } \\
\text { Interval of the } \\
\text { Difference }\end{array}$} & & & \\
\hline & & & & & Lower & Upper & & & \\
\hline Pair 1 & C1.3-C1.5 & .17576 & .89682 & .06982 & .03790 & .31361 & 2.517 & 164 & .013 \\
\hline Pair 2 & C1.2-C1.5 & -.13333 & 1.10174 & .08577 & -.30269 & .03602 & -1.555 & 164 & .122 \\
\hline Pair 3 & C1.1-C1.2 & -.21212 & 1.26292 & .09832 & -.40625 & -.01799 & -2.157 & 164 & .032 \\
\hline Pair 4 & C1.1 - C1.4 & .29697 & 1.41518 & .11017 & .07943 & .51451 & 2.696 & 164 & .008 \\
\hline Pair 5 & C1.1-C1.2 & -.21212 & 1.26292 & .09832 & -.40625 & -.01799 & -2.157 & 164 & .032 \\
\hline Pair 6 & $C 4.1-C 4.4$ & .21212 & .82483 & .06421 & .08533 & .33891 & 3.303 & 164 & .001 \\
\hline Pair 7 & C4.3-C4.4 & -.27273 & 1.29428 & .10076 & -.47168 & -.07377 & -2.707 & 164 & .008 \\
\hline Pair 8 & C4.2 - C4.3 & -.48171 & 1.37226 & .10716 & -.69330 & -.27012 & -4.495 & 163 & .000 \\
\hline
\end{tabular}

\section{E.3.3. Detailed Results of Reliability Analysis}

Table E.32: Research Question 2: Reliability Statistics for Items in C1

\begin{tabular}{|c|c|c|}
\hline & $\begin{array}{c}\text { Cronbach's } \\
\text { Alpha Based } \\
\text { on } \\
\begin{array}{c}\text { Cronbach's } \\
\text { Alpha }\end{array}\end{array}$ & \\
\hline .819 & $\begin{array}{c}\text { Standardized } \\
\text { Items }\end{array}$ & N of Items \\
\hline .834 & 5 \\
\hline
\end{tabular}

Table E.33: Research Question 2: Inter-Item Correlation Matrix for Items in C1

\begin{tabular}{|l|r|r|r|r|r|}
\hline & \multicolumn{1}{|c|}{$\mathrm{C} 1.1$} & \multicolumn{1}{c|}{$\mathrm{C} 1.2$} & \multicolumn{1}{c|}{$\mathrm{C} 1.3$} & \multicolumn{1}{c|}{$\mathrm{C} 1.4$} & \multicolumn{1}{c|}{$\mathrm{C} 1.5$} \\
\hline $\mathrm{C} 1.1$ & 1.000 & .458 & .549 & .451 & .352 \\
$\mathrm{C} 1.2$ & .458 & 1.000 & .697 & .375 & .503 \\
$\mathrm{C} 1.3$ & .549 & .697 & 1.000 & .504 & .635 \\
$\mathrm{C} 1.4$ & .451 & .375 & .504 & 1.000 & .486 \\
$\mathrm{C} 1.5$ & .352 & .503 & .635 & .486 & 1.000 \\
\hline
\end{tabular}

267 
Table E.34: Research Question 2: Reliability Statistics for Items in C2

\begin{tabular}{|r|c|r|}
\hline & $\begin{array}{c}\text { Cronbach's } \\
\text { Alpha Based } \\
\text { on } \\
\begin{array}{c}\text { Cronbach's } \\
\text { Alpha }\end{array}\end{array}$ & \\
\hline .799 & $\begin{array}{c}\text { Standardized } \\
\text { ltems }\end{array}$ & N of Items \\
\hline
\end{tabular}

Table E.35: Research Question 2: Inter-Item Correlation Matrix for Items in C2

\begin{tabular}{|l|r|r|}
\hline & \multicolumn{1}{|c|}{ C2.1 } & \multicolumn{1}{c|}{ C2.2 } \\
\hline C2.1 & 1.000 & .665 \\
C2.2 & .665 & 1.000 \\
\hline
\end{tabular}

Table E.36: Research Question 2: Reliability Statistics for Items in C4

\begin{tabular}{|c|c|c|}
\hline $\begin{array}{c}\text { Cronbach's } \\
\text { Alpha }\end{array}$ & $\begin{array}{c}\text { Cronbach's } \\
\text { Alpha Based } \\
\text { on } \\
\text { Standardized } \\
\text { Items } \\
\end{array}$ & $\mathrm{N}$ of ltems \\
\hline .786 & .799 & 4 \\
\hline
\end{tabular}

Table E.37: Research Question 2: Inter-Item Correlation Matrix for Items in C4

\begin{tabular}{|l|r|r|r|r|}
\hline & \multicolumn{1}{|c|}{$\mathrm{C} 4.1$} & \multicolumn{1}{c|}{ C4.2 } & \multicolumn{1}{c|}{ C4.3 } & \multicolumn{1}{c|}{ C4.4 } \\
\hline C4.1 & 1.000 & .488 & .514 & .682 \\
C4.2 & .488 & 1.000 & .472 & .415 \\
C4.3 & .514 & .472 & 1.000 & .415 \\
C4.4 & .682 & .415 & .415 & 1.000 \\
\hline
\end{tabular}

\section{E.4. Research Question 3}

The detailed statistical analysis results obtained while investigating the data corresponding to Research Question 3 are presented in this Section. 


\section{E.4.1. Detailed Descriptive Statistics}

Table E.38: Research Question 3: Detailed Descriptive Statistics

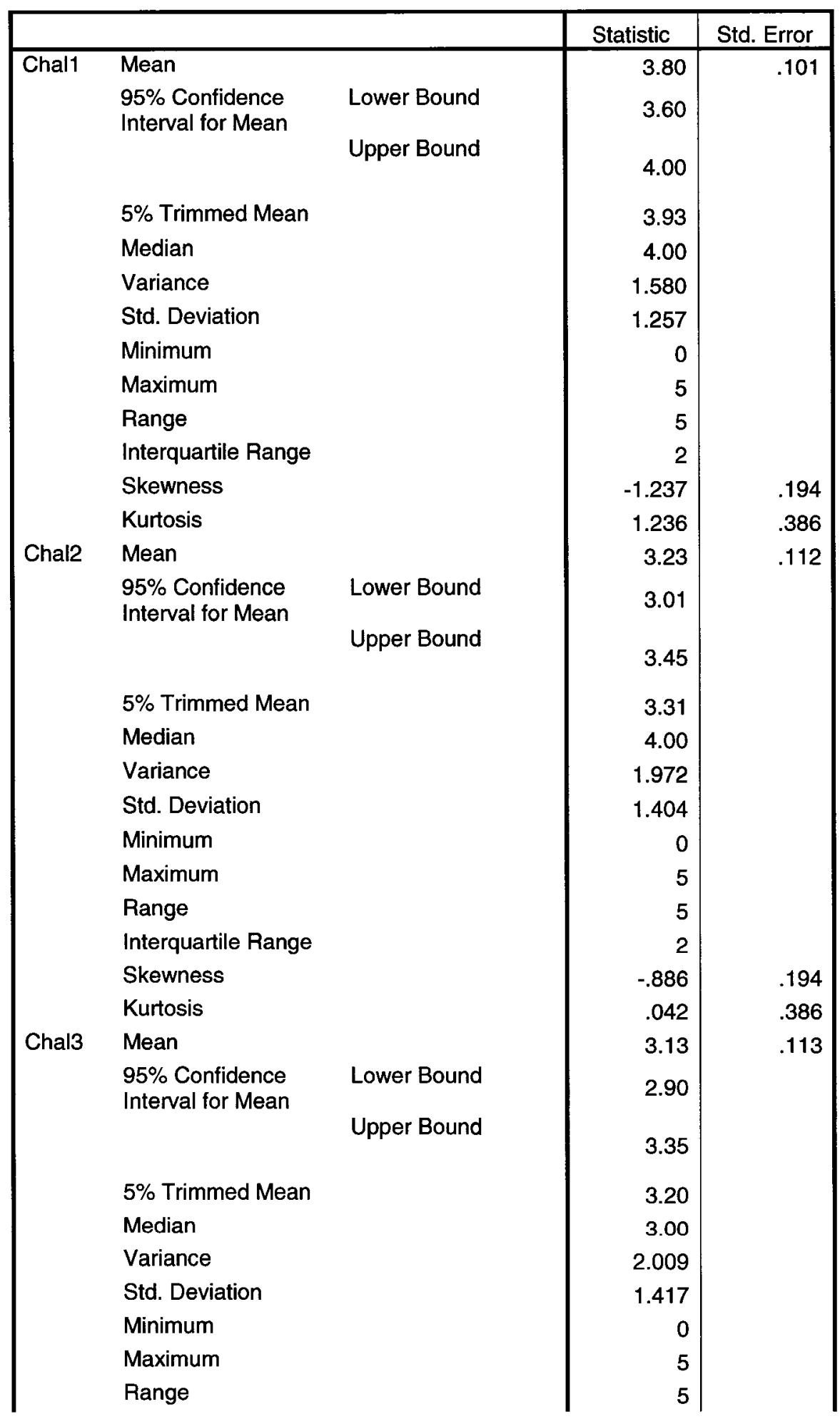




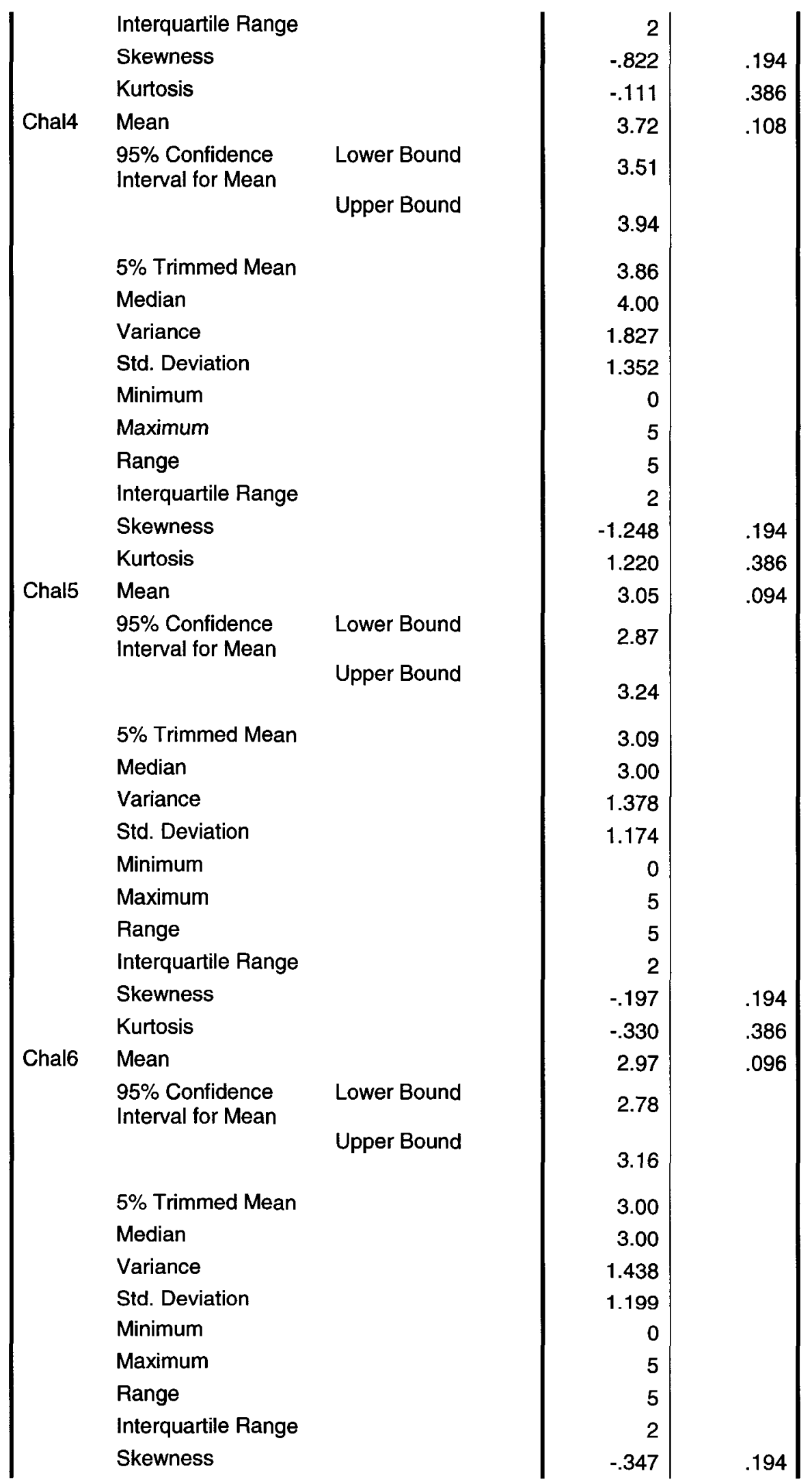




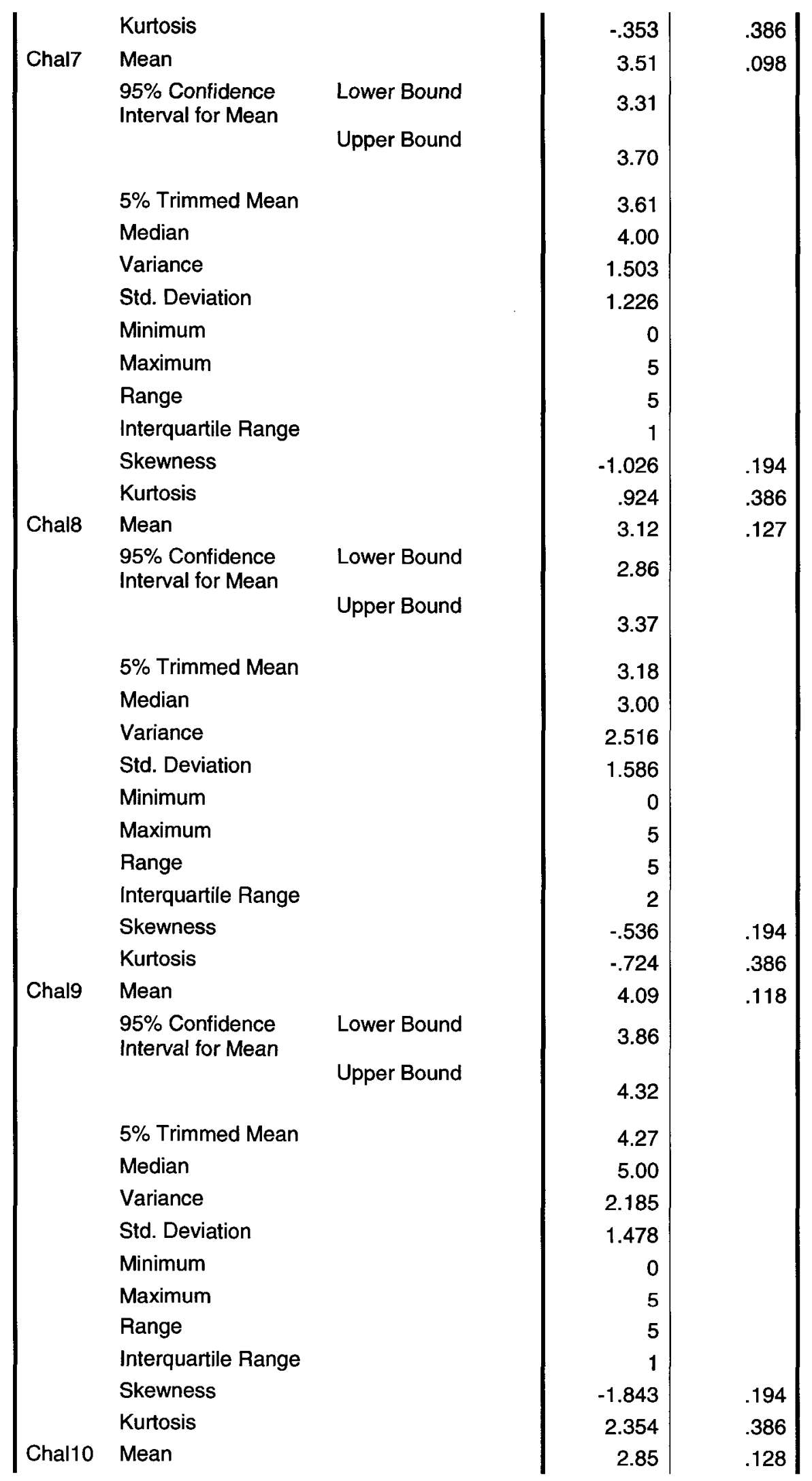




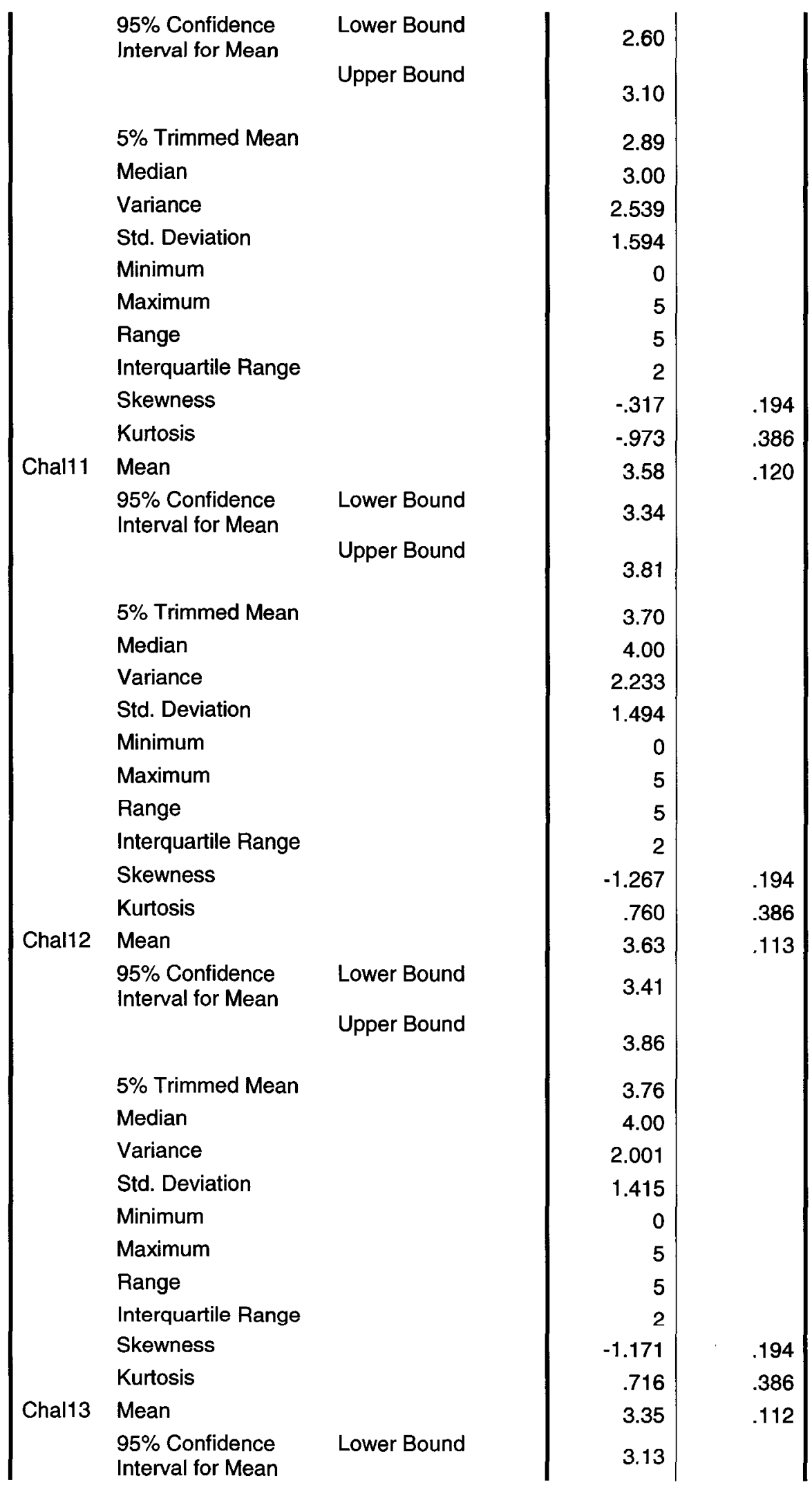




\begin{tabular}{|c|c|c|c|c|}
\hline & & Upper Bound & 357 & \\
\hline & $5 \%$ Trimmed Mean & & 3.45 & \\
\hline & Median & & 4.00 & \\
\hline & Variance & & 1.946 & \\
\hline & Std. Deviation & & 1.395 & \\
\hline & Minimum & & 0 & \\
\hline & Maximum & & 5 & \\
\hline & Range & & 5 & \\
\hline & Interquartile Range & & 1 & \\
\hline & Skewness & & -.970 & .194 \\
\hline & Kurtosis & & .324 & .386 \\
\hline Chal14 & Mean & & 3.32 & .119 \\
\hline & $\begin{array}{l}95 \% \text { Confidence } \\
\text { Interval for Mean }\end{array}$ & Lower Bound & 3.09 & \\
\hline & & Upper Bound & 3.55 & \\
\hline & $5 \%$ Trimmed Mean & & 3.41 & \\
\hline & Median & & 4.00 & \\
\hline & Variance & & 2.193 & \\
\hline & Std. Deviation & & 1.481 & \\
\hline & Minimum & & 0 & \\
\hline & Maximum & & 5 & \\
\hline & Range & & 5 & \\
\hline & Interquartile Range & & 1 & \\
\hline & Skewness & & -.917 & .194 \\
\hline & Kurtosis & & -.018 & .386 \\
\hline Chal15 & Mean & & 3.38 & .113 \\
\hline & $\begin{array}{l}\text { 95\% Confidence } \\
\text { Interval for Mean }\end{array}$ & Lower Bound & 3.16 & \\
\hline & & Upper Bound & 3.61 & \\
\hline & $5 \%$ Trimmed Mean & & 3.48 & \\
\hline & Median & & 4.00 & \\
\hline & Variance & & 2.006 & \\
\hline & Std. Deviation & & 1.416 & \\
\hline & Minimum & & 0 & \\
\hline & Maximum & & 5 & \\
\hline & Range & & 5 & \\
\hline & Interquartile Range & & 1 & \\
\hline & Skewness & & -.984 & .194 \\
\hline & Kurtosis & & .288 & .386 \\
\hline Chal16 & Mean & & 2.83 & .135 \\
\hline & $\begin{array}{l}95 \% \text { Confidence } \\
\text { Interval for Mean }\end{array}$ & Lower Bound & 2.57 & \\
\hline & & Upper Bound & 3.10 & \\
\hline
\end{tabular}

273 


\begin{tabular}{|c|c|c|c|c|}
\hline & $5 \%$ Trimmed Mean & & 2.87 & \\
\hline & Median & & 3.00 & \\
\hline & Variance & & 2.824 & \\
\hline & Std. Deviation & & 1.680 & \\
\hline & Minimum & & 0 & \\
\hline & Maximum & & 5 & \\
\hline & Range & & 5 & \\
\hline & Interquartile Range & & 2 & \\
\hline & Skewness & & -.502 & .194 \\
\hline & Kurtosis & & -.944 & .386 \\
\hline Chal17 & Mean & & 3.52 & .107 \\
\hline & $\begin{array}{l}95 \% \text { Confidence } \\
\text { Interval for Mean }\end{array}$ & Lower Bound & 3.31 & \\
\hline & & Upper Bound & 3.73 & \\
\hline & $5 \%$ Trimmed Mean & & 3.63 & \\
\hline & Median & & 4.00 & \\
\hline & Variance & & 1.800 & \\
\hline & Std. Deviation & & 1.342 & \\
\hline & Minimum & & 0 & \\
\hline & Maximum & & 5 & \\
\hline & Range & & 5 & \\
\hline & Interquartile Range & & 1 & \\
\hline & Skewness & & -1.097 & .194 \\
\hline & Kurtosis & & .801 & .386 \\
\hline Chal18 & Mean & & 3.12 & .113 \\
\hline & $\begin{array}{l}95 \% \text { Confidence } \\
\text { Interval for Mean }\end{array}$ & Lower Bound & 2.89 & \\
\hline & & Upper Bound & 3.34 & \\
\hline & $5 \%$ Trimmed Mean & & 3.18 & \\
\hline & Median & & 3.00 & \\
\hline & Variance & & 2.000 & \\
\hline & Std. Deviation & & 1.414 & \\
\hline & Minimum & & 0 & \\
\hline & Maximum & & 5 & \\
\hline & Range & & 5 & \\
\hline & Interquartile Range & & 2 & \\
\hline & Skewness & & -.651 & .194 \\
\hline & Kurtosis & & -.306 & .386 \\
\hline Chal19 & Mean & & 3.18 & .110 \\
\hline & $\begin{array}{l}95 \% \text { Confidence } \\
\text { Interval for Mean }\end{array}$ & Lower Bound & 2.96 & \\
\hline & & Upper Bound & 3.40 & \\
\hline & $5 \%$ Trimmed Mean & & 3.24 & \\
\hline & Median & & 3.00 & \\
\hline
\end{tabular}

274 
Variance

Std. Deviation

Minimum

Maximum

Range

Interquartile Range

Skewness

Kurtosis 


\section{E.4.2. Inter-Item Correlation}

Table E.39: Research Question 3: Inter-item Correlation Matrix

Inter-Item Corrolation Matrix

\begin{tabular}{|c|c|c|c|c|c|c|c|c|c|c|c|c|c|c|c|c|c|c|c|}
\hline & Chal1 & Chal2 & Chal3 & Chal4 & Chal5 & Chal6 & Chal7 & Chals & Chal9 & Chal10 & Chal11 & Chal12 & Chal13 & Chal14 & Chal15 & Chal16 & Chal17 & Chal18 & Chal19 \\
\hline Chal1 & 1.000 & .545 & .434 & .260 & .129 & .253 & .388 & .393 & .239 & .249 & .168 & .304 & .316 & .395 & .395 & .204 & .291 & .340 & \\
\hline Chal2 & .545 & 1.000 & .565 & . 234 & 267 & 288 & .501 & . 466 & 291 & .433 & . 342 & .452 & 288 & .479 & .338 & .271 & 391 & . 165 & .148 \\
\hline Chal3 & .434 & .565 & 1.000 & . 322 & .229 & .253 & . 419 & .473 & . 404 & .468 & .312 & .400 & .326 & .466 & .438 & .383 & . 385 & 211 & .222 \\
\hline Chal4 & .260 & .234 & .322 & 1.000 & .322 & 241 & .338 & .352 & .355 & 257 & .411 & .369 & .223 & .325 & .322 & .426 & .275 & .283 & .317 \\
\hline Chais & . 129 & 267 & .229 & .322 & 1.000 & .327 & . 421 & . 264 & . 075 & .487 & . 325 & 326 & .347 & .465 & .357 & 250 & .315 & .261 & 285 \\
\hline Chal6 & .253 & .288 & .253 & .241 & .327 & 1.000 & .362 & .263 & .205 & .328 & .316 & . 267 & .300 & . 275 & .212 & 183 & .259 & .269 & .300 \\
\hline Chal7 & .388 & .501 & .419 & .338 & .421 & .362 & 1.000 & .411 & .220 & .362 & . 304 & . 368 & .370 & . 422 & . 329 & . 264 & . 318 & .338 & .316 \\
\hline Chals & .393 & . 466 & .473 & .352 & .264 & .263 & .411 & 1.000 & . 384 & . 418 & . 402 & .364 & 355 & . 454 & . 477 & 349 & .414 & .259 & . 368 \\
\hline Chal9 & .239 & 291 & .404 & . 355 & .075 & .205 & .220 & .384 & 1.000 & .400 & .452 & .463 & .332 & .308 & .455 & .416 & .500 & .177 & . 166 \\
\hline Chal10 & .249 & .433 & .468 & .257 & .487 & .328 & . 362 & .418 & .400 & 1.000 & .418 & 397 & 299 & .504 & . 486 & .345 & .483 & 314 & 282 \\
\hline Chal11 & .168 & . 342 & .312 & .411 & .325 & .316 & . 304 & .402 & .452 & .418 & 1.000 & .671 & 289 & .359 & .355 & .406 & .422 & .246 & .341 \\
\hline Chal12 & . 304 & .452 & .400 & .369 & . 326 & .267 & .368 & . 364 & .463 & 397 & .671 & 1.000 & .432 & .527 & .418 & .427 & .532 & 399 & . 325 \\
\hline Chal13 & .316 & 288 & 326 & .223 & .347 & .300 & .370 & .355 & .332 & 299 & .289 & .432 & 1.000 & .495 & .457 & .328 & . 381 & .411 & .312 \\
\hline Chal14 & . 395 & . 479 & .466 & .325 & .465 & .275 & .422 & .454 & .308 & .504 & .359 & .527 & .495 & 1.000 & .547 & .439 & .458 & .343 & .328 \\
\hline Chal15 & . 395 & .338 & .438 & .322 & 357 & .212 & .329 & .477 & .455 & . 486 & . 355 & .418 & .457 & .547 & 1.000 & . 358 & .431 & .355 & . 351 \\
\hline Chal16 & .204 & . 271 & .383 & .426 & .250 & . 183 & .264 & . 349 & .416 & . 345 & .406 & .427 & .328 & .439 & .358 & 1.000 & .396 & 299 & .280 \\
\hline Chal17 & 291 & 391 & 385 & .275 & .315 & 259 & .318 & .414 & .500 & .483 & .422 & .532 & 381 & .458 & .431 & .396 & 1.000 & .455 & .347 \\
\hline Chal18 & .340 & . 165 & 211 & .283 & 261 & .269 & .338 & 259 & . 177 & .314 & .246 & 399 & .411 & .343 & . 355 & 299 & .455 & 1.000 & .429 \\
\hline Chal19 & .352 & .148 & .222 & .317 & .285 & .300 & .316 & .368 & .166 & .282 & 341 & .325 & .312 & .328 & .351 & .280 & .347 & .429 & 1.000 \\
\hline
\end{tabular}

The covariance matrix is calculated and used in the analysis. 


\section{E.4.3. t-Test}

Table E.40: Research Question 3: Paired Samples t-Test of Challenge Variables

\begin{tabular}{|c|c|c|c|c|c|c|c|c|c|}
\hline & & \multicolumn{5}{|c|}{ Paired Differences } & \multirow[b]{3}{*}{$\mathrm{t}$} & \multirow[b]{3}{*}{$d f$} & \multirow[b]{3}{*}{ Sig. (2-tailed) } \\
\hline & & \multirow[b]{2}{*}{ Mean } & \multirow{2}{*}{$\begin{array}{c}\text { Std. } \\
\text { Deviation }\end{array}$} & \multirow{2}{*}{$\begin{array}{l}\text { Std. Error } \\
\text { Mean }\end{array}$} & \multicolumn{2}{|c|}{$\begin{array}{l}95 \% \text { Confidence } \\
\text { Interval of the } \\
\text { Difference }\end{array}$} & & & \\
\hline & & & & & Lower & Upper & & & \\
\hline Pair 1 & Chal1 - Chal9 & -.292 & 1.672 & .132 & -.552 & -.032 & -2.215 & 160 & .028 \\
\hline Pair 2 & Chal1 - Chal4 & .087 & 1.587 & .125 & -.160 & .334 & .695 & 160 & .488 \\
\hline Pair 3 & $\begin{array}{l}\text { Chal4 - } \\
\text { Chal12 }\end{array}$ & .088 & 1.552 & .123 & -.155 & .331 & .715 & 158 & .476 \\
\hline Pair 4 & $\begin{array}{l}\text { Chal11 - } \\
\text { Chal12 }\end{array}$ & -.044 & 1.182 & .094 & -.229 & .141 & -.470 & 158 & .639 \\
\hline Pair 5 & $\begin{array}{l}\text { Chal11 - } \\
\text { Chal17 }\end{array}$ & .057 & 1.531 & .121 & -.183 & .296 & .466 & 158 & .642 \\
\hline Pair 6 & $\begin{array}{l}\text { Chal7 - } \\
\text { Chal17 }\end{array}$ & -.025 & 1.509 & .120 & -.262 & .211 & -.210 & 158 & .834 \\
\hline Pair 7 & $\begin{array}{l}\text { Chal7 - } \\
\text { Chal15 }\end{array}$ & .113 & 1.534 & .122 & -.127 & .354 & .930 & 158 & .354 \\
\hline Pair 8 & $\begin{array}{l}\text { Chal13 - } \\
\text { Chal15 }\end{array}$ & -.050 & 1.462 & .116 & -.279 & .179 & -.434 & 158 & .665 \\
\hline Pair 9 & $\begin{array}{l}\text { Chal13 - } \\
\text { Chal14 }\end{array}$ & .019 & 1.443 & .114 & -.207 & .245 & .165 & 158 & .869 \\
\hline $\begin{array}{l}\text { Pair } \\
10\end{array}$ & $\begin{array}{l}\text { Chal2 - } \\
\text { Chal14 }\end{array}$ & -.057 & 1.481 & .117 & -.289 & .175 & -.482 & 158 & .630 \\
\hline
\end{tabular}




\section{Appendix F \\ Summary of Survey Responses}

In this Appendix, we present the consolidated summary (in percentage figures) of responses

corresponding to the different Likert scale items obtained from the survey.

\section{F.1. Success Factors Survey}

Table F.1: Summary of Success Factor Survey Responses

\begin{tabular}{|c|c|c|c|c|c|c|}
\hline & 1 & 2 & 3 & 4 & 5 & $x$ \\
\hline Ind1 & $0 \%$ & $1 \%$ & $1 \%$ & $20 \%$ & $77 \%$ & $1 \%$ \\
\hline Ind2 & $2 \%$ & $9 \%$ & $10 \%$ & $40 \%$ & $38 \%$ & $1 \%$ \\
\hline Ind3 & $3 \%$ & $10 \%$ & $14 \%$ & $38 \%$ & $33 \%$ & $1 \%$ \\
\hline Ind4 & $0 \%$ & $3 \%$ & $12 \%$ & $38 \%$ & $46 \%$ & $1 \%$ \\
\hline \multicolumn{7}{|l|}{ Ind5 } \\
\hline Ind5a & $14 \%$ & $15 \%$ & $5 \%$ & $17 \%$ & $49 \%$ & $0 \%$ \\
\hline Ind5b & $28 \%$ & $23 \%$ & $11 \%$ & $17 \%$ & $15 \%$ & $5 \%$ \\
\hline Ind6 & $3 \%$ & $2 \%$ & $4 \%$ & $11 \%$ & $79 \%$ & $1 \%$ \\
\hline \multicolumn{7}{|l|}{ Ind7 } \\
\hline Ind7a & $1 \%$ & $6 \%$ & $7 \%$ & $22 \%$ & $63 \%$ & $0 \%$ \\
\hline Ind7b & $2 \%$ & $7 \%$ & $9 \%$ & $25 \%$ & $56 \%$ & $0 \%$ \\
\hline Ind7c & $4 \%$ & $7 \%$ & $8 \%$ & $33 \%$ & $47 \%$ & $1 \%$ \\
\hline Ind7d & $1 \%$ & $7 \%$ & $14 \%$ & $35 \%$ & $42 \%$ & $1 \%$ \\
\hline Ind7e & $4 \%$ & $10 \%$ & $7 \%$ & $27 \%$ & $50 \%$ & $1 \%$ \\
\hline Ind7f & $6 \%$ & $13 \%$ & $15 \%$ & $31 \%$ & $34 \%$ & $0 \%$ \\
\hline Ind7g & $10 \%$ & $12 \%$ & $8 \%$ & $22 \%$ & $47 \%$ & $0 \%$ \\
\hline Ind7h & $4 \%$ & $14 \%$ & $12 \%$ & $29 \%$ & $38 \%$ & $2 \%$ \\
\hline Ind 8 & $9 \%$ & $14 \%$ & $15 \%$ & $35 \%$ & $26 \%$ & $1 \%$ \\
\hline Ind9 & $4 \%$ & $10 \%$ & $16 \%$ & $31 \%$ & $31 \%$ & $8 \%$ \\
\hline Ind10 & $1 \%$ & $9 \%$ & $11 \%$ & $38 \%$ & $41 \%$ & $0 \%$ \\
\hline \multicolumn{7}{|l|}{$\begin{array}{l}\text { Ind11 } \\
\end{array}$} \\
\hline Ind11a & $1 \%$ & $11 \%$ & $15 \%$ & $46 \%$ & $27 \%$ & $0 \%$ \\
\hline Ind11b & $0 \%$ & $1 \%$ & $6 \%$ & $25 \%$ & $68 \%$ & $0 \%$ \\
\hline Ind11c & $0 \%$ & $2 \%$ & $7 \%$ & $39 \%$ & $50 \%$ & $0 \%$ \\
\hline Ind11d & $1 \%$ & $4 \%$ & $10 \%$ & $38 \%$ & $47 \%$ & $0 \%$ \\
\hline Ind11e & $1 \%$ & $3 \%$ & $6 \%$ & $32 \%$ & $57 \%$ & $1 \%$ \\
\hline Ind11f & $1 \%$ & $6 \%$ & $7 \%$ & $26 \%$ & $59 \%$ & $0 \%$ \\
\hline \multicolumn{7}{|l|}{ Ind12 } \\
\hline Ind12a & $5 \%$ & $6 \%$ & $12 \%$ & $37 \%$ & $38 \%$ & $1 \%$ \\
\hline Ind12b & $6 \%$ & $13 \%$ & $9 \%$ & $31 \%$ & $39 \%$ & $1 \%$ \\
\hline Ind12c & $7 \%$ & $16 \%$ & $9 \%$ & $31 \%$ & $36 \%$ & $1 \%$ \\
\hline Ind12d & $6 \%$ & $15 \%$ & $8 \%$ & $24 \%$ & $46 \%$ & $1 \%$ \\
\hline Ind12e & $2 \%$ & $2 \%$ & $10 \%$ & $41 \%$ & $45 \%$ & $0 \%$ \\
\hline \multicolumn{7}{|l|}{ Ind13 } \\
\hline Ind13a & $1 \%$ & $9 \%$ & $14 \%$ & $41 \%$ & $31 \%$ & $4 \%$ \\
\hline Ind $13 b$ & $0 \%$ & $11 \%$ & $13 \%$ & $43 \%$ & $28 \%$ & $5 \%$ \\
\hline Ind13c & $1 \%$ & $8 \%$ & $18 \%$ & $36 \%$ & $30 \%$ & $6 \%$ \\
\hline Ind13d & $2 \%$ & $10 \%$ & $14 \%$ & $36 \%$ & $35 \%$ & $2 \%$ \\
\hline Ind14 & $2 \%$ & $3 \%$ & $11 \%$ & $39 \%$ & $45 \%$ & $0 \%$ \\
\hline \multicolumn{7}{|c|}{ Dependent } \\
\hline D1 & $1 \%$ & $6 \%$ & $13 \%$ & $37 \%$ & $38 \%$ & $4 \%$ \\
\hline$D 2$ & $1 \%$ & $3 \%$ & $12 \%$ & $30 \%$ & $47 \%$ & $6 \%$ \\
\hline D3 & $0 \%$ & $2 \%$ & $7 \%$ & $24 \%$ & $64 \%$ & $2 \%$ \\
\hline D4 & $0 \%$ & $2 \%$ & $4 \%$ & $20 \%$ & $71 \%$ & $1 \%$ \\
\hline$\overline{D 5}$ & $2 \%$ & $5 \%$ & $19 \%$ & $32 \%$ & $38 \%$ & $4 \%$ \\
\hline
\end{tabular}




\section{F.2. Changes Survey}

Table F.2: Summary of Changes Survey Responses

\begin{tabular}{|c|c|c|c|c|c|c|}
\hline & 1 & 2 & 3 & 4 & 5 & $X$ \\
\hline \multicolumn{7}{|l|}{ C1 } \\
\hline C1.1 & $1 \%$ & $2 \%$ & $13 \%$ & $32 \%$ & $46 \%$ & $6 \%$ \\
\hline C1.2 & $2 \%$ & $2 \%$ & $11 \%$ & $29 \%$ & $53 \%$ & $3 \%$ \\
\hline $\mathrm{C} 1.3$ & $0 \%$ & $1 \%$ & $3 \%$ & $24 \%$ & $69 \%$ & $3 \%$ \\
\hline C1.4 & $2 \%$ & $5 \%$ & $13 \%$ & $39 \%$ & $33 \%$ & $6 \%$ \\
\hline $\mathrm{C} 1.5$ & $1 \%$ & $3 \%$ & $6 \%$ & $21 \%$ & $65 \%$ & $4 \%$ \\
\hline \multicolumn{7}{|l|}{ C2 } \\
\hline C2.1 & $1 \%$ & $2 \%$ & $3 \%$ & $23 \%$ & $67 \%$ & $4 \%$ \\
\hline $\mathrm{C} 2.2$ & $1 \%$ & $3 \%$ & $7 \%$ & $37 \%$ & $49 \%$ & $4 \%$ \\
\hline C3 & $1 \%$ & $5 \%$ & $11 \%$ & $52 \%$ & $26 \%$ & $5 \%$ \\
\hline \multicolumn{7}{|l|}{ C4 } \\
\hline C4.1 & $1 \%$ & $1 \%$ & $3 \%$ & $15 \%$ & $77 \%$ & $3 \%$ \\
\hline $\mathrm{C} 4.2$ & $3 \%$ & $6 \%$ & $16 \%$ & $42 \%$ & $26 \%$ & $6 \%$ \\
\hline $\mathrm{C} 4.3$ & $0 \%$ & $5 \%$ & $7 \%$ & $33 \%$ & $48 \%$ & $6 \%$ \\
\hline C4.4 & $0 \%$ & $2 \%$ & $7 \%$ & $27 \%$ & $60 \%$ & $3 \%$ \\
\hline
\end{tabular}

\section{F.3. Challenges Survey}

Table F.3: Summary of Challenges Survey Responses

\begin{tabular}{|l|c|c|c|c|c|c|}
\hline & $\mathbf{1}$ & $\mathbf{2}$ & $\mathbf{3}$ & $\mathbf{4}$ & $\mathbf{5}$ & $\mathbf{X}$ \\
\hline Chal1 & $4 \%$ & $8 \%$ & $14 \%$ & $38 \%$ & $32 \%$ & $4 \%$ \\
\hline Chal2 & $5 \%$ & $14 \%$ & $19 \%$ & $40 \%$ & $15 \%$ & $7 \%$ \\
\hline Chal3 & $7 \%$ & $10 \%$ & $25 \%$ & $36 \%$ & $13 \%$ & $8 \%$ \\
\hline Chal4 & $2 \%$ & $5 \%$ & $20 \%$ & $32 \%$ & $34 \%$ & $7 \%$ \\
\hline Chal5 & $3 \%$ & $29 \%$ & $26 \%$ & $27 \%$ & $11 \%$ & $3 \%$ \\
\hline Chal6 & $7 \%$ & $25 \%$ & $23 \%$ & $32 \%$ & $9 \%$ & $4 \%$ \\
\hline Chal7 & $3 \%$ & $9 \%$ & $24 \%$ & $38 \%$ & $21 \%$ & $4 \%$ \\
\hline Chal8 & $5 \%$ & $17 \%$ & $16 \%$ & $25 \%$ & $24 \%$ & $12 \%$ \\
\hline Chal9 & $2 \%$ & $2 \%$ & $7 \%$ & $25 \%$ & $\mathbf{5 5 \%}$ & $9 \%$ \\
\hline Chal10 & $10 \%$ & $17 \%$ & $21 \%$ & $22 \%$ & $18 \%$ & $13 \%$ \\
\hline Chal11 & $2 \%$ & $6 \%$ & $14 \%$ & $41 \%$ & $27 \%$ & $10 \%$ \\
\hline Chal12 & $3 \%$ & $8 \%$ & $16 \%$ & $37 \%$ & $29 \%$ & $7 \%$ \\
\hline Chal13 & $6 \%$ & $8 \%$ & $23 \%$ & $35 \%$ & $19 \%$ & $8 \%$ \\
\hline Chal14 & $6 \%$ & $8 \%$ & $21 \%$ & $35 \%$ & $22 \%$ & $8 \%$ \\
\hline Chal15 & $6 \%$ & $8 \%$ & $23 \%$ & $36 \%$ & $19 \%$ & $7 \%$ \\
\hline Chal16 & $5 \%$ & $14 \%$ & $20 \%$ & $27 \%$ & $17 \%$ & $17 \%$ \\
\hline Chal17 & $5 \%$ & $7 \%$ & $21 \%$ & $37 \%$ & $23 \%$ & $8 \%$ \\
\hline Chal18 & $8 \%$ & $12 \%$ & $26 \%$ & $31 \%$ & $15 \%$ & $9 \%$ \\
\hline Chal19 & $11 \%$ & $16 \%$ & $22 \%$ & $28 \%$ & $19 \%$ & $5 \%$ \\
\hline
\end{tabular}

